\title{
Organocatalytic Enantioselective Vinylogous Aldol Reaction of Allyl Aryl Ketones to Activated Acyclic Ketones
}

Zhenzhong Jing, Xiangbin Bai, Wenchao Chen, Gao Zhang, Bo Zhu, and Zhiyong Jiang*

Key Laboratory of Natural Medicine and Immuno-Engineering of Henan Province, Henan University, Jinming Campus, Kaifeng, Henan, P. R. China, 475004

Email: chmjzy@henu.edu.cn 


\section{Supporting Information}

\section{Table of Contents}

1. General information

2. Preparation and characterization of catalysts II and III

3. Divergent synthesis of $(\alpha-\delta)-\mathrm{CF}_{3}-\mathrm{THCAs}$

4. Synthetic procedure and characterization of adducts

5. Determination of the absolute configuration by $X$-ray crystallography

6. Plausible transition state

7. Copies of NMR spectra 


\section{General information}

\section{General Procedures and Methods}

Experiments involving moisture and/or air sensitive components were performed under a positive pressure of nitrogen in oven-dried glassware equipped with a rubber septum inlet. Dried solvents and liquid reagents were transferred by oven-dried syringes or hypodermic syringe cooled to ambient temperature in a desiccator. Reactions mixtures were stirred in 10 $\mathrm{mL}$ sample vial with Teflon-coated magnetic stirring bars unless otherwise stated. Moisture in non-volatile reagents/compounds was removed in high vacuo by means of an oil pump and subsequent purging with nitrogen. Solvents were removed in vacuo under $\sim 30 \mathrm{mmHg}$ and heated with a water bath at $30-35^{\circ} \mathrm{C}$ using Changcheng rotary evaporator with Changcheng aspirator. The condenser was cooled with running water at $0{ }^{\circ} \mathrm{C}$.

All experiments were monitored by analytical thin layer chromatography (TLC). TLC was performed on pre-coated plates, $60 \mathrm{~F}_{254}$. After elution, plate was visualized under UV illumination at $254 \mathrm{~nm}$ for UV active material. Further visualization was achieved by staining $\mathrm{KMnO}_{4}$, ceric molybdate, or anisaldehyde solution. For those using the aqueous stains, the TLC plates were heated on a hot plate.

Columns for flash chromatography (FC) contained silica gel 200-300 mesh. Columns were packed as slurry of silica gel in petroleum ether and equilibrated solution using the appropriate solvent system. The elution was assisted by applying pressure of about $2 \mathrm{~atm}$ with an air pump.

\section{$\underline{\text { Instrumentations }}$}

Proton nuclear magnetic resonance $\left({ }^{1} \mathrm{H}\right.$ NMR), carbon NMR $\left({ }^{13} \mathrm{C}\right.$ NMR), and fluorous $\left({ }^{19} \mathrm{~F}\right.$ NMR) spectra were recorded in $\mathrm{CDCl}_{3}$ otherwise stated. Chemical shifts are reported in parts per million (ppm), using the residual solvent signal as an internal standard: $\mathrm{CDCl}_{3}\left({ }^{1} \mathrm{H}\right.$ NMR: $\delta 7.26$, singlet; ${ }^{13} \mathrm{C}$ NMR: $\delta 77.0$, triplet). Multiplicities were given as: $s$ (singlet), $d$ (doublet), $t$ (triplet), $q$ (quartet), quintet, $m$ (multiplets), $d d$ (doublet of doublets), $d t$ (doublet of triplets), and $b r$ (broad). Coupling constants $(J)$ were recorded in Hertz $(\mathrm{Hz})$. The number of proton atoms $(n)$ for a given resonance was indicated by $n \mathrm{H}$. The number of carbon atoms $(n)$ for a given resonance was indicated by $n$ C. HRMS was reported in units of mass of charge ratio $\left(\mathrm{m} / \mathrm{z}\right.$ ). Mass samples were dissolved in $\mathrm{CH}_{3} \mathrm{CN}$ (HPLC Grade) unless otherwise stated. 
Optical rotations were recorded on a polarimeter with a sodium lamp of wavelength $589 \mathrm{~nm}$ and reported as follows; $[\alpha]_{\lambda}^{T^{\circ} \mathrm{C}}(\mathrm{c}=\mathrm{g} / 100 \mathrm{~mL}$, solvent). Melting points were determined on a melting point apparatus.

Enantiomeric excesses were determined by chiral High Performance Liquid Chromatography (HPLC) analysis. UV detection was monitored at $254 \mathrm{~nm}, 230 \mathrm{~nm}$ and $210 \mathrm{~nm}$ at the same time. HPLC samples were dissolved in HPLC grade isopropanol (IPA) unless otherwise stated.

\section{$\underline{\text { Materials }}$}

All commercial reagents were purchased with the highest purity grade. They were used without further purification unless specified. All solvents used, mainly petroleum ether (PE) and ethyl acetate (EtOAc) were distilled. Anhydrous DCM and $\mathrm{CH}_{3} \mathrm{CN}$ were freshly distilled from $\mathrm{CaH}_{2}$ and stored under $\mathrm{N}_{2}$ atmosphere. THF, $\mathrm{Et}_{2} \mathrm{O}$, tert-butylbenzene and toluene were freshly distilled from sodium/benzophenone before use. Anhydrous methanol and etanol were distilled from Mg. All compounds synthesized were stored in a $-20{ }^{\circ} \mathrm{C}$ freezer and light-sensitive compounds were protected with aluminium foil. 


\section{Preparation and characterization of catalysts II and III}

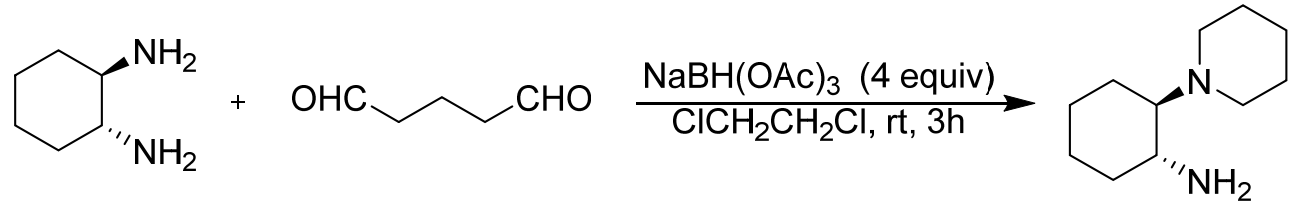

Glutaraldehyde ( $50 \mathrm{wt} \%$ in $\mathrm{H}_{2} \mathrm{O}, 0.93 \mathrm{~mL}, 5.1 \mathrm{mmol}$ ) was added dropwise into a mixture of $(1 R, 2 R)$-cyclohexane-1,2-diamine $(560 \mathrm{mg}, 4.9 \mathrm{mmol})$ and $\mathrm{NaBH}(\mathrm{OAc})_{3}(4.16 \mathrm{~g}, 19.6 \mathrm{mmol})$ in $\mathrm{ClCH}_{2} \mathrm{CH}_{2} \mathrm{Cl}(30 \mathrm{~mL})$ at room temperature. The resulting mixture was stirred at room temperature for $3 \mathrm{~h}$, and quenched with $\mathrm{NaOH}$ aq solution $(6 \mathrm{~N}, 15 \mathrm{~mL})$. The organic layer was separated and the aqueous layer was extracted with $\mathrm{CH}_{2} \mathrm{Cl}_{2}(3 \times 15 \mathrm{~mL})$. The combined organic layers were concentrated. The residue was dissolved in $20 \mathrm{~mL} \mathrm{CH}_{2} \mathrm{Cl}_{2}$, washed with brine $(10 \mathrm{~mL})$, dried over $\mathrm{Na}_{2} \mathrm{SO}_{4}$, filtered, and concentrated to give $794 \mathrm{mg}$ product as a yellow liquid ( $89 \%$ yield).<smiles>N[C@@H]1CCCCC1N1CCCCC1</smiles><smiles>O=[N+]([O-])c1cc(C(F)(F)F)cc(C(F)(F)F)c1</smiles>

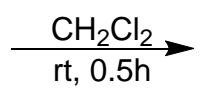<smiles>FC(F)(F)c1cc(NC(=S)N[C@H]2CCCCC2N2CCCCC2)cc(C(F)(F)F)c1</smiles>

The solution of 1-isothiocyanato-3,5-bis (trifluoromethyl) benzene (325.4 mg, $1.20 \mathrm{mmol}$ ) in $5 \mathrm{~mL} \mathrm{CH}_{2} \mathrm{Cl}_{2}$ was added dropwise into a solution of $(1 R, 2 R)$-2-(piperidin-1-yl) cyclohexanamine in $\mathrm{CH}_{2} \mathrm{Cl}_{2}$ at room temperature. The resulting mixture was stirred at room temperature for $0.5 \mathrm{~h}$ and monitored by TLC. Upon complete consumption of $(1 R$, 2R)-2-(piperidin-1-yl) cyclohexanamine, the reaction mixture was evaporated to give the crude product, which was purified by flash chromatography with dichloromethane/methanol (120:1-60:1) to afford white solid II in 76\% yield.<smiles>FC(F)(F)c1cc(NC(=S)N[C@H]2CCCCC2N2CCCCC2)cc(C(F)(F)F)c1</smiles>

II

${ }^{1} \mathrm{H}$ NMR (300 MHz, CD 3 OD) $\delta 8.19$ (s, 2H), 7.63 (s, 1H), $4.22(\mathrm{t}, J=9.6 \mathrm{~Hz}, 1 \mathrm{H}), 2.75(\mathrm{~s}, 2 \mathrm{H}), 2.54-2.40(\mathrm{~m}, 4 \mathrm{H})$, 1.99-1.69 (m, 3H), 1.57-1.43 (m, 7H), 1.36-1.13 (m, 5H); ${ }^{13} \mathrm{C}$ NMR $\left(75 \mathrm{MHz}, \mathrm{CD}_{3} \mathrm{OD}\right) \delta 181.6,143.1,132.8\left(\mathrm{q},{ }^{2} J_{\mathrm{C}-\mathrm{F}}=\right.$ $33.0 \mathrm{~Hz}), 124.8$ (q, $\left.{ }^{1} J_{\mathrm{C}-\mathrm{F}}=270.2 \mathrm{~Hz}\right) 123.5,117.7$ (two peaks), 117.6, 69.0, 56.1, 50.6, 33.5, 27.6, 26.5, 25.90 (two peaks), 24.7. 


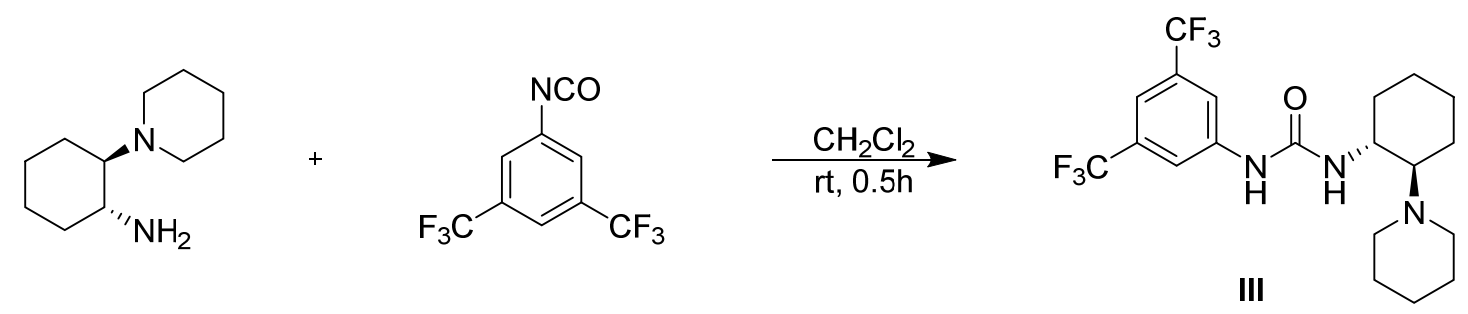

The solution of 1-isocyanato-3,5-bis (trifluoromethyl) benzene (306.1 mg, $1.20 \mathrm{mmol}$ ) in 5 $\mathrm{mL} \mathrm{CH}_{2} \mathrm{Cl}_{2}$ was added dropwise into a solution of $(1 R, 2 R)$-2-(piperidin-1-yl) cyclohexanamine $(182.3 \mathrm{mg}, 1 \mathrm{mmol})$ in $\mathrm{CH}_{2} \mathrm{Cl}_{2}$ at room temperature. The resulting mixture was stirred at room temperature for $0.5 \mathrm{~h}$ and monitored by TLC. Upon complete consumption of $(1 R, 2 R)-2$-(piperidin-1-yl) cyclohexanamine, the reaction mixture was evaporated to give the crude product, which was purified by flash chromatography with dichloromethane/methanol (120:1-60:1) to afford white solid III in $82 \%$ yield.<smiles>O=C(Nc1cc(C(F)(F)F)cc(C(F)(F)F)c1)N[C@H]1CCCC[C@H]1N1CCCCC1</smiles>

III

${ }^{1} \mathrm{H}$ NMR $\left(300 \mathrm{MHz},\left(\mathrm{CD}_{3}\right)_{2} \mathrm{CO}\right) \delta 8.85(\mathrm{~s}, 1 \mathrm{H}), 8.16(\mathrm{~s}, 2 \mathrm{H})$, $7.50(\mathrm{~s}, 1 \mathrm{H}), 6.12(\mathrm{~s}, 1 \mathrm{H}), 3.47(\mathrm{t}, J=10.5 \mathrm{~Hz}, 1 \mathrm{H}), 2.65-2.64$ $(\mathrm{m}, 2 \mathrm{H}), 2.45(\mathrm{~d}, J=11.9 \mathrm{~Hz}, 1 \mathrm{H}), 2.34-2.25(\mathrm{~m}, 3 \mathrm{H})$, $1.89-1.77(\mathrm{~m}, 3 \mathrm{H}), 1.50-1.05(\mathrm{~m}, 10 \mathrm{H}) ;{ }^{13} \mathrm{C} \mathrm{NMR}(75 \mathrm{MHz}$, Acetone) $\delta 156.1,143.9,132.4\left(\mathrm{q},{ }^{2} J_{\mathrm{C}-\mathrm{F}}=32.6 \mathrm{~Hz}\right), 124.5(\mathrm{q}$, ${ }^{1} J_{\mathrm{C}-\mathrm{F}}=270.3 \mathrm{~Hz}$ ), 118.2 (two peaks), 114.6, 114.5 (two peaks), 114.4 (two peaks), 68.7, 51.8, $50.0,34.2,27.4,26.3,25.6,25.4,23.9$. 


\section{Divergent synthesis of $(\alpha-\delta)-C_{3}-$ THCAs}

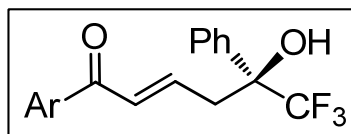

3u: $\mathrm{Ar}=4-\mathrm{MeOPh}$ $94 \%$ ee a) $\mathrm{H}_{2}, 10 \% \mathrm{Pd} / \mathrm{C}, \mathrm{MeOH}, 10 \mathrm{~min}$ b) $m$-CPBA, $\mathrm{Na}_{2} \mathrm{HPO}_{4}, \mathrm{CH}_{2} \mathrm{Cl}_{2}$ $\mathrm{rt}, 4 \mathrm{~h}, 86 \%$ yield of two steps<smiles>O=C(CCC[C@](O)(c1ccccc1)C(F)(F)F)O[Ga]</smiles><smiles>O[C@@](CCCCc1ccccc1)(c1ccccc1)C(F)(F)F</smiles>

8: $>99 \%$ ee

\section{c) $\mathrm{H}_{2}, 30 \% \mathrm{Pd} / \mathrm{C}$} $\mathrm{MeOH}, \mathrm{rt}, 6 \mathrm{~h}, 96 \%$ yield

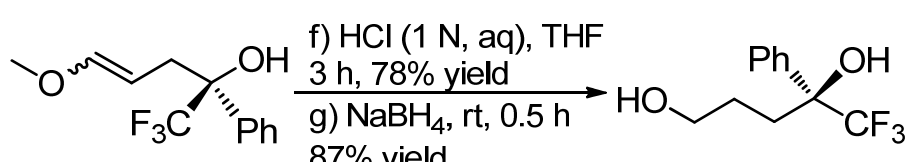

10 $87 \%$ yield

e) $\mathrm{CICH}_{3} \mathrm{OCH}_{2} \mathrm{PPh}_{3}$, NaHMDS THF, $-78^{\circ} \mathrm{C}, 53 \%$ yield

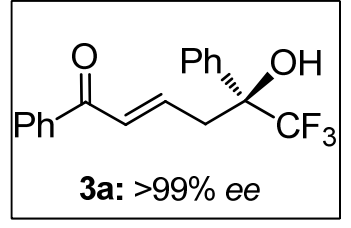
d) $\mathrm{O}_{3}, \mathrm{CH}_{2} \mathrm{Cl}_{2}$ $78^{\circ} \mathrm{C}, 5 \mathrm{~h}$, then $\underset{\mathrm{PPh}_{3}, \mathrm{rt}, 8 \mathrm{~h}}{\stackrel{-78}{ }}$ $86 \%$ yield

i) $\mathrm{PhMgBr}, \mathrm{THF}$ $0^{\circ} \mathrm{C}$ to rt $62 \%$ yield j) $\mathrm{CrO}_{3}, \mathrm{H}_{2} \mathrm{SO}_{4}$ (conc) $\mathrm{H}_{2} \mathrm{O}, \mathrm{Et}_{2} \mathrm{O}$ $\checkmark 0^{\circ} \mathrm{C}, 92 \%$ yield

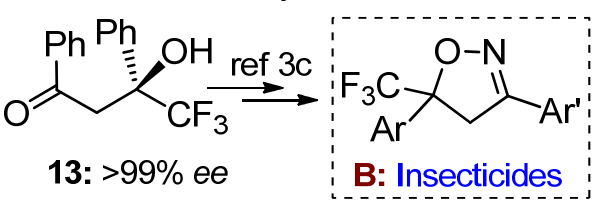<smiles>CO[C@@](C(=O)O)(c1ccccc1)C(F)(F)F</smiles>

m) TFA, rt, $0.5 \mathrm{~h}$

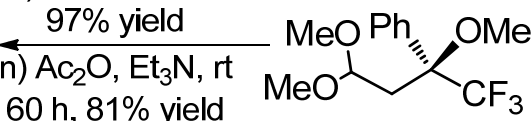
A: $>99 \%$ ee $\alpha-\mathrm{CF}_{3}-\mathrm{THCA}$ $60 \mathrm{~h}, 81 \%$ yield

o) $\mathrm{O}_{3}$ then 14 h) $\mathrm{CrO}_{3}, \mathrm{H}_{2} \mathrm{SO}_{4}$ (conc) $\mathrm{H}_{2} \mathrm{O}, \mathrm{Et}_{2} \mathrm{O}, 0^{\circ} \mathrm{C}$ $92 \%$ yield 11: $>99 \%$ ee $\gamma-\mathrm{CF}_{3}-\mathrm{THCA}$
9

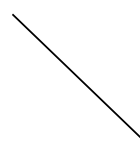

k) $\left(\mathrm{CH}_{3} \mathrm{O}\right)_{3} \mathrm{CH}, p-\mathrm{TsOH}$ $\mathrm{CH}_{2} \mathrm{Cl}_{2}, \mathrm{rt}, 0.5 \mathrm{~h}, 93 \%$ yield

I) $\mathrm{NaH}, \mathrm{Mel}, \mathrm{THF}$

$0{ }^{\circ} \mathrm{C}$ to $\mathrm{rt}, 91 \%$ yield

12: $>99 \%$ ee $\beta-\mathrm{CF}_{3}-\mathrm{THCA}$ $\mathrm{CrO}_{3}, \mathrm{H}_{2} \mathrm{SO}_{4}$ (conc) $\mathrm{H}_{2} \mathrm{O}, 2 \mathrm{~h}, 64 \%$ yield

a) $3 \mathrm{u}(140.2 \mathrm{mg}, 0.4 \mathrm{mmol})$ was dissolved in dry $\mathrm{MeOH}(10 \mathrm{~mL})$ and added to a dry round-bottom flask containing $10 \% \mathrm{Pd} / \mathrm{C}(14.0 \mathrm{mg}, 10 \% \mathrm{w} / \mathrm{w})$. The reaction mixture was purged with $\mathrm{H}_{2}$ gas and the $\mathrm{H}_{2}$ atmosphere was maintained with a $\mathrm{H}_{2}$ balloon, stirred at room temperature. The reaction was monitored by TLC. Upon completion of reaction (about 10 $\min$ ), the $\mathrm{Pd} / \mathrm{C}$ was removed by suction filtration and the solvent was evaporated off under reduced pressure. After removing the solvent, product was obtained as white solid and the solid was used directly to the next step without purification.

b) The above solid was dissolved in $\mathrm{CH}_{2} \mathrm{Cl}_{2}(3 \mathrm{~mL})$. Disodium hydrogen phosphate (142.0 $\mathrm{mg}, 1.0 \mathrm{mmol}, 2.5$ equiv) and $m$-CPBA (172.6 mg, $1.0 \mathrm{mmol}, 2.5$ equiv) were added and the reaction mixture was stirred at room temperature for $4 \mathrm{~h}$. The mixture was poured into water $(5 \mathrm{~mL})$ and saturated aq. sodium hydrogen carbonate $(5 \mathrm{~mL})$, the organic layer was separated 
and the aqueous phase was extracted with $\mathrm{CH}_{2} \mathrm{Cl}_{2}(3 \times 10 \mathrm{~mL})$. The combined organic layers were washed with water $(20 \mathrm{~mL})$, dried $\left(\mathrm{Na}_{2} \mathrm{SO}_{4}\right)$ and evaporated to give the crude product, which was purified by flash chromatography with petroleum ether/ethyl acetate mixture $(5: 1)$ to afford white solid 7 (121.2 $\mathrm{mg})$ in $86 \%$ yield for two steps and $95 \%$ ee.

c) $3 \mathrm{a}(128.1 \mathrm{mg}, 0.4 \mathrm{mmol})$ was dissolved in dry $\mathrm{MeOH}(10 \mathrm{~mL})$ and added to a dry round bottom flask containing $30 \% \mathrm{Pd} / \mathrm{C}(10 \% \mathrm{w} / \mathrm{w})$. The reaction mixture was purged with $\mathrm{H}_{2}$ gas and the $\mathrm{H}_{2}$ atmosphere was maintained with a $\mathrm{H}_{2}$ balloon. The reaction was stirred at room temperature for $6 \mathrm{~h}$, then $\mathrm{Pd} / \mathrm{C}$ was removed by suction filtration and the solvent was evaporated off under reduced pressure to give the crude product which was purified by flash chromatography with petroleum ether/ethyl acetate mixture (10:1) to afford the colorless oil $\mathbf{8}$ $(118.4 \mathrm{mg}$ ) in $96 \%$ yield and $>99 \% e e$.

d) To a solution of 3a $(320.3 \mathrm{mg}, 1 \mathrm{mmol})$ in $\mathrm{CH}_{2} \mathrm{Cl}_{2}(30 \mathrm{~mL})$ at $-78{ }^{\circ} \mathrm{C}, \mathrm{O}_{3}$ was bubbled until the reaction was complete (about 5 hours, determined by TLC). Then, the reaction mixture was allowed to warm to room temperature, triphenylphosphine (1049.2 $\mathrm{mg}, 4 \mathrm{mmol})$ was added, and the mixture was kept stirring for 8 hours. After that, the solvent was removed under reduced pressure following purification of the crude product mixture by column chromatography on silica gel using petroleum ether/ethyl acetate mixture (20/1-10/1 ratio) to afford the white solid 9 ( $275.5 \mathrm{mg}$ ) in $86 \%$ yield.

e) NaHMDS ( $1 \mathrm{M}$ in THF, $1.14 \mathrm{~mL}, 1.14 \mathrm{mmol})$ was added dropwise to a solution of $\mathrm{Ph}_{3} \mathrm{PCH}_{2} \mathrm{OCH}_{3} \mathrm{Cl}(411.4 \mathrm{mg}, 1.2 \mathrm{mmol})$ in THF $(4 \mathrm{~mL})$ at $-78{ }^{\circ} \mathrm{C}$ for 20 minutes. The reaction mixture was allowed to warm to room temperature and stirred for $1 \mathrm{~h}$ at $0^{\circ} \mathrm{C}$ and cooled down to $-78^{\circ} \mathrm{C}$. Then a solution of aldehyde $9(130.9 \mathrm{mg}, 0.6 \mathrm{mmol})$ in THF $(2 \mathrm{~mL})$ was added dropwise for 10 minutes. The resulting mixture was allowed to warm to room temperature and stirred 4 hours. Water $(0.5 \mathrm{ml})$ was added then the reaction mixture was concentrated under reduced pressure and the residue was extracted with ethyl ether $(3 \times 15$ $\mathrm{mL}$ ). The combined organic layers were dried over anhydrous $\mathrm{Na}_{2} \mathrm{SO}_{4}$, filtered and concentrated under reduced pressure. The crude material was purified by column chromatography on silica gel using petroleum ether/ethyl acetate mixture (30/1-20/1 ratio) to afford white solid $\mathbf{1 0}(130.5 \mathrm{mg})$ in $53 \%$ yield.

f) $1 \mathrm{~N} \mathrm{HCl}$ aq solution $(4 \mathrm{~mL})$ was added to a solution of the previously prepared methyl enol 
ether $10(61.6 \mathrm{mg}, 0.25 \mathrm{mmol})$ in THF $(2 \mathrm{~mL})$ at room temperature. After stirring for 3 hours, THF was evaporated under reduced pressure and the aqueous phase was extracted with ethyl ether $(3 \times 15 \mathrm{~mL})$. Combined organic layers were dried over anhydrous $\mathrm{Na}_{2} \mathrm{SO}_{4}$, filtered and concentrated under reduced pressure. The crude material was purified by column chromatography on silica gel using petroleum ether/ethyl acetate mixture (30/1-15/1 ratio) to afford colorless oil (181.1 mg) in 78\% yield.

g) To a solution of the corresponding aldehyde $(46.5 \mathrm{mg}, 0.2 \mathrm{mmol})$ in $5 \mathrm{~mL}$ of methanol, $\mathrm{NaBH}_{4}$ (11.4 mg, $0.30 \mathrm{mmol}, 1.5$ equiv) was added. After stirring at room temperature for 0.5 hours, the reaction mixture was quenched with water $(3 \mathrm{~mL})$. After that, $10.0 \mathrm{~mL}$ ethyl ether was added, the aqueous phase was separated and extracted with ethyl ether $(3 \times 15 \mathrm{~mL})$. The combined organic layers were dried with $\mathrm{Na}_{2} \mathrm{SO}_{4}$. The solvent was removed under reduced pressure following purification of the crude product mixture by chromatography on silica gel using petroleum ether/ethyl acetate mixture (20/1-10/1 ratio) to afford the white solid 11 $(40.8 \mathrm{mg})$ in $87 \%$ yield and $>99 \%$ ee.

h) To dissolved the obtained aldehyde $9(87.3 \mathrm{mg}, 0.4 \mathrm{mmol})$ in $\mathrm{Et}_{2} \mathrm{O}(5.0 \mathrm{~mL})$, and the solution was cooled to $0{ }^{\circ} \mathrm{C}$, then Jones reagents ${ }^{1}(0.5 \mathrm{~mL})$ was added dropwise for 20 minutes. The mixture was kept stirring for 1 hours at $0^{\circ} \mathrm{C}$ until the reaction was complete (determined by TLC), then the reaction mixture was extracted with $\mathrm{CH}_{2} \mathrm{Cl}_{2}(3 \times 10 \mathrm{~mL})$. The combined organic layers were dried with $\mathrm{Na}_{2} \mathrm{SO}_{4}$. The solvent was removed under reduced pressure following purification of the crude product mixture by chromatography on silica gel using petroleum ether/ethyl acetate mixture (1/1 ratio) to afford white solid $12(86.2 \mathrm{mg})$ in $92 \%$ yield and $>99 \%$ ee.

i) To a solution of aldehyde 9 (174.6 $\mathrm{mg}, 0.8 \mathrm{mmol})$ in anhydrous THF (5 mL) was added dropwise phenylmagnesium bromide (1.0 M solution in THF, $2.4 \mathrm{~mL})$ at $0{ }^{\circ} \mathrm{C}$ for 30 minutes. Then, the reaction mixture was allowed to warm to room temperature, and the mixture was kept stirring for 18 hours. After TLC analysis indicated a complete conversion, the reaction was quenched with a saturated aqueous solution of $\mathrm{NH}_{4} \mathrm{Cl}(5 \mathrm{~mL})$. The crude mixture was extracted with ethyl acetate $(3 \times 10 \mathrm{~mL})$. The combined organic phases were dried over $\mathrm{Na}_{2} \mathrm{SO}_{4}$ and purified by column chromatography on silica gel with petroleum ether/ethyl acetate mixture (10/1 ratio) to afford colorless oil (147.0 $\mathrm{mg})$ in $62 \%$ yield. 
j) To a solution of above product $(118.5 \mathrm{mg}, 0.4 \mathrm{mmol})$ in anhydrous $\mathrm{Et}_{2} \mathrm{O}(5 \mathrm{~mL})$ was added dropwise Jones reagents at $0{ }^{\circ} \mathrm{C}$ for 20 minutes. Then, the reaction mixture was allowed to warm to room temperature until the reaction was complete (about 0.5 hours, determined by TLC). The reaction mixture was extracted with $\mathrm{CH}_{2} \mathrm{Cl}_{2}(3 \times 10 \mathrm{~mL})$ and organic phases were dried over $\mathrm{Na}_{2} \mathrm{SO}_{4}$. After that, the solvent was removed under reduced pressure following purification of the crude product mixture by column chromatography on silica gel using petroleum ether/ethyl acetate mixture (20/1 ratio) to afford colorless oil $\mathbf{1 3}(82.4 \mathrm{mg})$ in $70 \%$ yield and $>99 \% e e$.

k) A solution of aldehyde $9(174.6 \mathrm{mg}, 0.8 \mathrm{mmol})$ in $\mathrm{CH}_{2} \mathrm{Cl}_{2}(10 \mathrm{~mL})$ was added p-toluenesulfonic acid $(41.3 \mathrm{mg}, 0.24 \mathrm{mmol})$ and trimethyl orthoformate $(127.4 \mathrm{mg}, 1.2$ mmol). The reaction mixture was stirred at room temperature until the addition product consumed (determined by TLC, about $0.5 \mathrm{~h}$ ) and then purified by column chromatography on silica gel using petroleum ether/ethyl acetate mixture (20/1 ratio) to afford colorless oil (196.6 $\mathrm{mg}$ ) in $93 \%$ yield.

l) A solution of (S)-1,1,1-trifluoro-4,4-dimethoxy-2-phenylbutan-2-ol (158.5 mg, $0.6 \mathrm{mmol})$ in dry tetrahydrofuran $(5 \mathrm{~mL})$ was added slowly to a suspension of sodium hydride $(\sim 60 \%$, $120 \mathrm{mg}, 3 \mathrm{mmol})$ in dry tetrahydrofuran $(10 \mathrm{~mL})$ at $0{ }^{\circ} \mathrm{C}$ under nitrogen gas. The resulting solution was then stirred for $45 \mathrm{~min}$. at room temperature and then iodomethane $(373.5 \mu \mathrm{L}, 6$ mmol) was added slowly into the reaction flask. The reaction mixture was further stirred for $20 \mathrm{~h}$ at room temperature under nitrogen gas. Afterwards, the reaction was quenched with water $(10 \mathrm{~mL})$ and extracted with ethyl acetate $(3 \times 10 \mathrm{~mL})$. The combined organic phases were washed once again with water $(20 \mathrm{~mL})$, brine solution $(20 \mathrm{~mL})$ and dried over anhydrous $\mathrm{Na}_{2} \mathrm{SO}_{4}$. The concentrated solution was evaporated off under reduced pressure and purified by column chromatography on silica gel with petroleum ether/ethyl acetate mixture (20/1 ratio) to afford colorless oil $\mathbf{1 4}(151.9 \mathrm{mg})$ in $91 \%$ yield.

m) The compound $14(139.1 \mathrm{mg}, 0.5 \mathrm{mmol})$ was dissolved in $5 \mathrm{~mL}$ of trifluoroacetic acid $/ \mathrm{H}_{2} \mathrm{O}$ $(1: 1, \mathrm{v} / \mathrm{v})$ at room temperature. The reaction mixture was further stirred for 30 minutes at room temperature. After that, the reaction was quenched with saturated sodium bicarbonate (5 $\mathrm{mL})$ and extracted with dichloromethane $(3 \times 10 \mathrm{~mL})$. The combined organic phases were dried over anhydrous $\mathrm{Na}_{2} \mathrm{SO}_{4}$ and evaporated off under reduced pressure and purified by 
column chromatography on silica gel with petroleum ether/ethyl acetate mixture (15/1 ratio) to afford colorless oil $(112.6 \mathrm{mg})$ in $97 \%$ yield.

n) To a solution of corresponding aldehyde $(116.1 \mathrm{mg}, 0.5 \mathrm{mmol})$, acetic anhydride $(255.2$ $\mathrm{mg}, 2.5 \mathrm{mmol})$ and triethylamine $(101.2 \mathrm{mg}, 1 \mathrm{mmol})$ was added dimethylaminopyridine (8.2 $\mathrm{mg}, 0.05 \mathrm{mmol})$. The solution was stirred at room temperature for 60 hours, then poured onto ice and the resulting mixture was stirred for an hour. The aqueous phase was separated and extracted with diethyl ether $(3 \times 10 \mathrm{~mL})$. The extract was washed with saturated aqueous sodium bicarbonate solution until neutral, then with water $(20 \mathrm{ml})$, brine $(10 \mathrm{~mL})$, and dried over anhydrous $\mathrm{Na}_{2} \mathrm{SO}_{4}$. Diethyl ether was removed under vacuum and the product was purified by column chromatography on silica gel using petroleum ether/ethyl acetate mixture (40/1 ratio) to afford the colorless oil $(111.1 \mathrm{mg})$ in $81 \%$ yield.

o) To a solution of $(S, E)-4,4,4$-trifluoro-3-methoxy-3-phenylbut-1-en-1-yl acetate (54.8 mg, $0.2 \mathrm{mmol})$ in $\mathrm{CH}_{2} \mathrm{Cl}_{2}(10 \mathrm{~mL})$ at $-78{ }^{\circ} \mathrm{C}, \mathrm{O}_{3}$ was bubbled until the reaction was complete (about 2 hours, determined by TLC). Then, $\mathrm{SMe}_{2}(0.1 \mathrm{~mL})$ was added at $-78{ }^{\circ} \mathrm{C}$, and the mixture was kept stirring for $1 \mathrm{~h}$. After that, the mixture was concentrated to afford aldehyde which was used for next step without purification. The obtained crude aldehyde was dissolved in $\mathrm{Et}_{2} \mathrm{O}(5.0 \mathrm{~mL})$, then Jones reagents $(1 \mathrm{~mL})$ was added, and the mixture was stirred at room temperature for $2 \mathrm{~h}$. After the reaction was complete (determined by TLC), the reaction mixture was extracted with diethyl ether $(3 \times 5 \mathrm{~mL})$, and the combined organic phases were carefully neutralized with $\mathrm{NaOH}$ aq solution $(2 \mathrm{~N})$ until $\mathrm{pH}$ value reached 13 . Then, $\mathrm{H}_{2} \mathrm{O}(10$ $\mathrm{mL})$ was added to the mixture and extracted with $\mathrm{H}_{2} \mathrm{O}(3 \times 5 \mathrm{~mL})$. The combined water phases were added $\mathrm{HCl}$ aq solution $(2 \mathrm{~N})$ until $\mathrm{pH}=2$ and extracted with DCM $(3 \times 5 \mathrm{~mL})$. The combined organic phases were dried over anhydrous $\mathrm{Na}_{2} \mathrm{SO}_{4}$ and evaporated off under reduced pressure to afford colorless oil (R)-Mosher's acid A (30.0 mg) in 64\% yield.

1. Jones reagents: $\mathrm{CrO}_{3}(26.72 \mathrm{~g})$ was dissolved in $\mathrm{H}_{2} \mathrm{SO}_{4}\left(23 \mathrm{~mL}\right.$, conc) at $0{ }^{\circ} \mathrm{C}$. Then the mixture was diluted by $\mathrm{H}_{2} \mathrm{O}$ to $100 \mathrm{~mL}$. 


\section{Synthetic procedure and characterization of adducts}<smiles>[R]C(=O)C=CCC([R2])(O)C([Y5])([R])C</smiles>

3a: $\mathrm{Ar}=\mathrm{Ph}$ $\mathrm{R}=\mathrm{Ph}$

Trifluoromethyl ketone 2 a (17.4 mg, $0.1 \mathrm{mmol}, 1.0$ equiv), $\mathrm{Na}_{3} \mathrm{PO}_{4}$

(32.8 mg, $0.2 \mathrm{mmol}, 2.0$ equiv) and catalyst IV ( $4.4 \mathrm{mg}, 0.01 \mathrm{mmol}$, 0.1 equiv) were dissolved in tert-butylbenzene $(1.0 \mathrm{~mL})$ and stirred at $-10{ }^{\circ} \mathrm{C}$ for $10 \mathrm{~min}$. Then allyl ketone $1 \mathrm{a}(43.9 \mathrm{mg}, 0.3 \mathrm{mmol}, 3.0$ equiv) was added. The reaction mixture was stirred at $-10{ }^{\circ} \mathrm{C}$ and monitored by TLC. Upon complete consumption of trifluoromethyl ketone $\mathbf{2 a}$, the reaction mixture was directly loaded onto a short silica gel column, followed by gradient elution with PE/EA mixture (20/1-5/1 ratio). Removing the solvent in vacuo, afforded product 3a. White solid, Mp $133.7-135.2{ }^{\circ} \mathrm{C}$; $29.8 \mathrm{mg}(0.1 \mathrm{mmol}), 93 \%$ yield; $95 \%$ ee; $[\alpha]_{\mathrm{D}}^{26}-36.4\left(c 2.47, \mathrm{CHCl}_{3}\right) ;{ }^{1} \mathrm{H} \mathrm{NMR}(300 \mathrm{MHz}$, $\left.\mathrm{CDCl}_{3}\right) \delta 7.75(\mathrm{~d}, J=7.4 \mathrm{~Hz}, 2 \mathrm{H}), 7.59-7.51(\mathrm{~m}, 3 \mathrm{H}), 7.46-7.33(\mathrm{~m}, 5 \mathrm{H}), 6.91(\mathrm{~d}, J=15.5$ $\mathrm{Hz}, 1 \mathrm{H}), 6.76-6.66(\mathrm{~m}, 1 \mathrm{H}), 3.25-3.05(\mathrm{~m}, 2 \mathrm{H}), 3.16(\mathrm{~s}, 1 \mathrm{H}) ;{ }^{13} \mathrm{C} \mathrm{NMR}\left(75 \mathrm{MHz}, \mathrm{CDCl}_{3}\right) \delta$ $190.4,140.6,137.1,135.8,133.0,130.7,128.9,128.6$ (two peaks), 128.5, 126.3, 125.2 (q, $J=$ $284.0 \mathrm{~Hz}), 77.7$ (q, $J=28.2 \mathrm{~Hz}), 39.0 ;{ }^{19} \mathrm{~F}$ NMR $\left(376 \mathrm{MHz}, \mathrm{CDCl}_{3}\right) \delta-79.55$; HRMS (ESI) $\mathrm{m} / \mathrm{z} 343.0928\left(\mathrm{M}+\mathrm{Na}^{+}\right)$, calc. for $\mathrm{C}_{18} \mathrm{H}_{15} \mathrm{~F}_{3} \mathrm{O}_{2} \mathrm{Na} 343.0922$.

The ee was determined by HPLC analysis. CHIRALPAK IB (4.6 mm i.d. x $250 \mathrm{~mm}$ ); Hexane/2-propanol $=90 / 10$; flow rate $1.0 \mathrm{~mL} / \mathrm{min} ; 25^{\circ} \mathrm{C} ; 254 \mathrm{~nm}$; retention time: $10.1 \mathrm{~min}$ (minor) and 11.1 min (major).
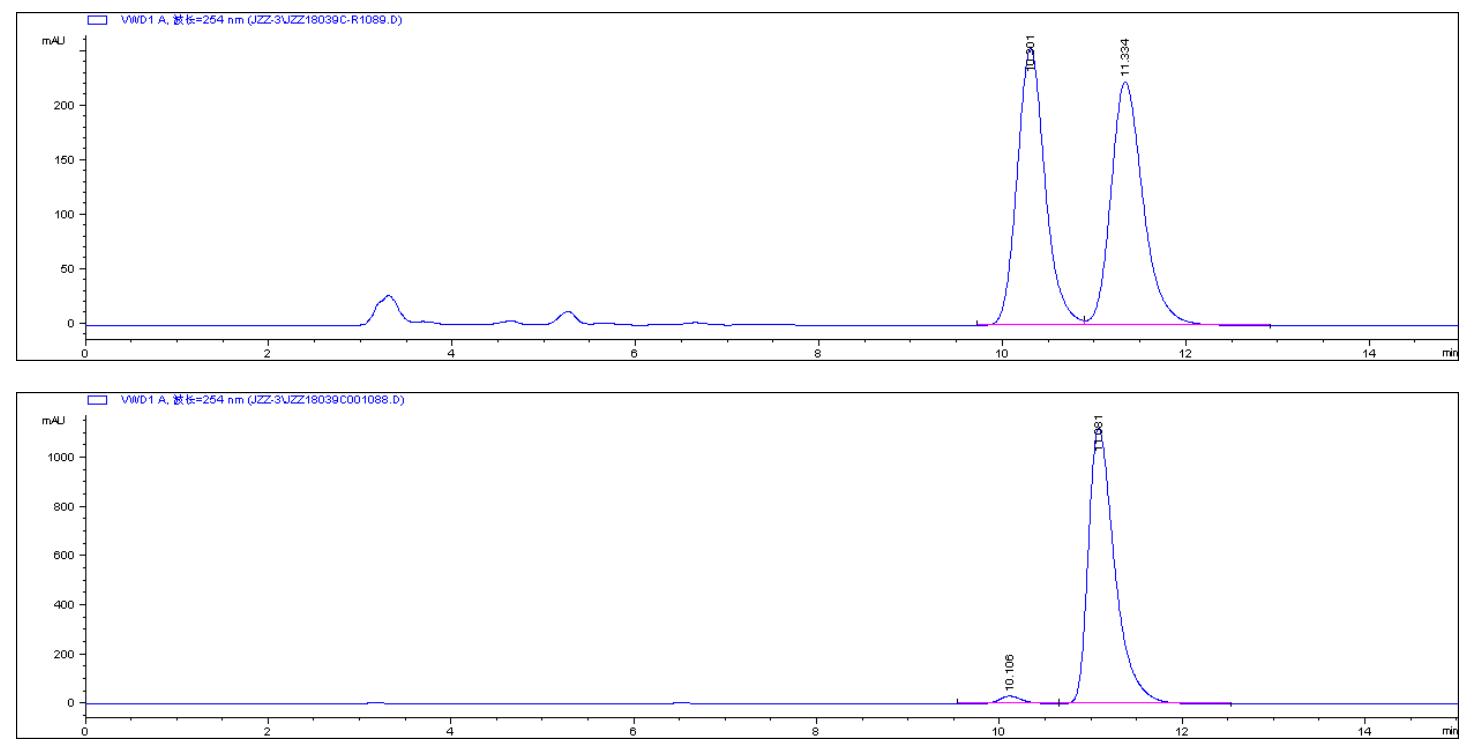
<smiles>[R]C(=O)/C=C/CC([R2])(O)C(F)(F)F</smiles>

3b: $\mathrm{Ar}=\mathrm{Ph}$

$\mathrm{R}=4-\mathrm{CF}_{3} \mathrm{Ph} \quad-10{ }^{\circ} \mathrm{C}$ for $10 \mathrm{~min}$. Then allyl ketone 1a $(43.9 \mathrm{mg}, 0.3 \mathrm{mmol}, 3.0$ equiv) was added. The reaction mixture was stirred at $-10{ }^{\circ} \mathrm{C}$ and monitored by TLC. Upon complete consumption of trifluoromethyl ketone $\mathbf{2} \mathbf{b}$, the reaction mixture was directly loaded onto a short silica gel column, followed by gradient elution with PE/EA mixture (20/1-5/1 ratio). Removing the solvent in vacuo, afforded product $\mathbf{3 b}$. White solid, Mp $88.2-90.9{ }^{\circ} \mathrm{C}$; $35.0 \mathrm{mg}(0.1 \mathrm{mml}), 90 \%$ yield; $92 \% e e ;[\alpha]_{\mathrm{D}}^{26}-26.3$ (c 2.47, $\left.\mathrm{CHCl}_{3}\right) ;{ }^{1} \mathrm{H}$ NMR $(300 \mathrm{MHz}$, $\left.\mathrm{CDCl}_{3}\right) \delta 7.75-7.66(\mathrm{~m}, 6 \mathrm{H}), 7.51(\mathrm{t}, J=7.4 \mathrm{~Hz}, 1 \mathrm{H}), 7.41(\mathrm{t}, J=7.8 \mathrm{~Hz}, 2 \mathrm{H}), 6.92(\mathrm{~d}, J=$ $15.5 \mathrm{~Hz}, 1 \mathrm{H}), 6.75-6.65(\mathrm{~m}, 1 \mathrm{H}), 3.59(\mathrm{~s}, 1 \mathrm{H}), 3.17(\mathrm{~m}, 2 \mathrm{H}) ;{ }^{13} \mathrm{C}$ NMR $\left(75 \mathrm{MHz}, \mathrm{CDCl}_{3}\right) \delta$ 190.3, 140.0, 139.7, 136.9, 133.3, 131.8, 131.3, 130.9, 130.6, 130.4, 129.2 (two peaks), 128.6, 128.2, 127.0, 126.8, 125.6 (two peaks), 125.5 (two peaks), 125.4, 123.0, 122.0, 118.4, 77.2, 76.8, 76.4, 76.0, 39.0; ${ }^{19} \mathrm{~F}$ NMR (376 MHz, $\left.\mathrm{CDCl}_{3}\right) \delta-62.77,-79.37$; HRMS (ESI) $\mathrm{m} / \mathrm{z}$ $411.0797\left(\mathrm{M}+\mathrm{Na}^{+}\right)$, calc. for $\mathrm{C}_{19} \mathrm{H}_{14} \mathrm{~F}_{6} \mathrm{O}_{2} \mathrm{Na} 411.0796$.

The ee was determined by HPLC analysis. CHIRALPAK IB (4.6 mm i.d. x $250 \mathrm{~mm}$ ); Hexane/2-propanol $=90 / 10$; flow rate $1.0 \mathrm{~mL} / \mathrm{min} ; 25{ }^{\circ} \mathrm{C} ; 254 \mathrm{~nm}$; retention time: $10.7 \mathrm{~min}$ (minor) and $14.3 \mathrm{~min}$ (major).
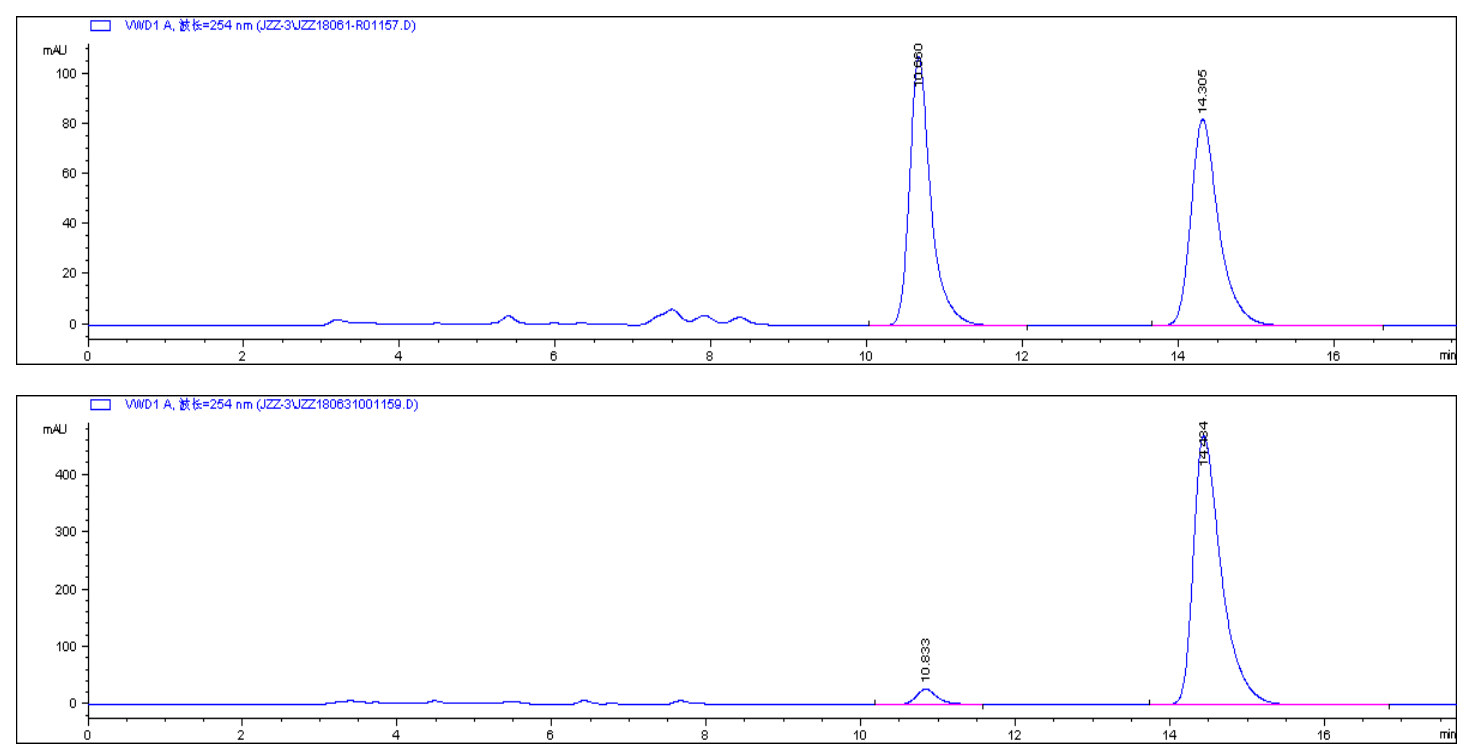
<smiles>[R]C(=O)/C=C/CC([R2])(O)C(F)(F)F</smiles>

Trifluoromethyl ketone $2 \mathbf{c}\left(19.2 \mathrm{mg}, 0.1 \mathrm{mmol}, 1.0\right.$ equiv), $\mathrm{Na}_{3} \mathrm{PO}_{4}$. (32.8 mg, $0.2 \mathrm{mmol}, 2.0$ equiv) and catalyst $\mathbf{I V}(4.4 \mathrm{mg}, 0.01 \mathrm{mmol}$, 3c: $\mathrm{Ar}=\mathrm{Ph}$ 0.1 equiv) were dissolved in tert-butylbenzene $(1.0 \mathrm{~mL})$ and stirred at $\mathrm{R}=4-\mathrm{FPh} \quad-10{ }^{\circ} \mathrm{C}$ for $10 \mathrm{~min}$. Then allyl ketone 1a $(43.9 \mathrm{mg}, 0.3 \mathrm{mmol}, 3.0$ equiv) was added. The reaction mixture was stirred at $-10{ }^{\circ} \mathrm{C}$ and monitored by TLC. Upon complete consumption of trifluoromethyl ketone $\mathbf{2 c}$, the reaction mixture was directly loaded onto a short silica gel column, followed by gradient elution with PE/EA mixture (20/1-5/1 ratio). Removing the solvent in vacuo, afforded product 3c. White solid, Mp 107.0-108.3 ${ }^{\circ} \mathrm{C}$; $30.1 \mathrm{mg}(0.1 \mathrm{mmol}), 89 \%$ yield; $95 \%$ ee; $[\alpha]_{\mathrm{D}}^{26}-31.0\left(\right.$ c $\left.2.42, \mathrm{CHCl}_{3}\right) ;{ }^{1} \mathrm{H}$ NMR (300 MHz, $\left.\mathrm{CDCl}_{3}\right) \delta 7.77(\mathrm{~d}, 2 \mathrm{H}), 7.78-7.52(\mathrm{~m}, 3 \mathrm{H}), 7.41(\mathrm{t}, J=7.6 \mathrm{~Hz}, 2 \mathrm{H}), 7.09(\mathrm{t}, J=8.64 \mathrm{~Hz}, 2 \mathrm{H})$, $6.92(\mathrm{~d}, J=15.5 \mathrm{~Hz}, 1 \mathrm{H}), 6.76-6.66(\mathrm{~m}, 1 \mathrm{H}), 3.47(\mathrm{~s}, 1 \mathrm{H}), 3.22-3.04(\mathrm{~m}, 2 \mathrm{H}) ;{ }^{13} \mathrm{C}$ NMR $(75$ $\mathrm{MHz}, \mathrm{CDCl}_{3}$ ) $\delta 190.3,164.5,161.2,140.3,137.03,133.2,131.8,131.7,130.7,128.6$ (two peaks), 128.5 (two peaks), 128.4 (two peaks), 125.1 (q, $J=283.4 \mathrm{~Hz}$ ), 123.19 (s), 115.6, 115.4, $76.4(\mathrm{q}, J=28.4 \mathrm{~Hz}), 38.9 ;{ }^{19} \mathrm{~F}$ NMR $\left(376 \mathrm{MHz}, \mathrm{CDCl}_{3}\right) \delta-79.80,-112.84$; HRMS (ESI) $\mathrm{m} / \mathrm{z} 361.0829\left(\mathrm{M}+\mathrm{Na}^{+}\right)$, calc. for $\mathrm{C}_{18} \mathrm{H}_{14} \mathrm{~F}_{4} \mathrm{O}_{2} \mathrm{Na} 361.0828$.

The ee was determined by HPLC analysis. CHIRALPAK IB (4.6 mm i.d. x $250 \mathrm{~mm})$; Hexane $/ 2$-propanol $=95 / 05$; flow rate $1.0 \mathrm{~mL} / \mathrm{min} ; 25{ }^{\circ} \mathrm{C} ; 254 \mathrm{~nm}$; retention time: $19.2 \mathrm{~min}$ (major) and $21.2 \mathrm{~min}$ (minor).
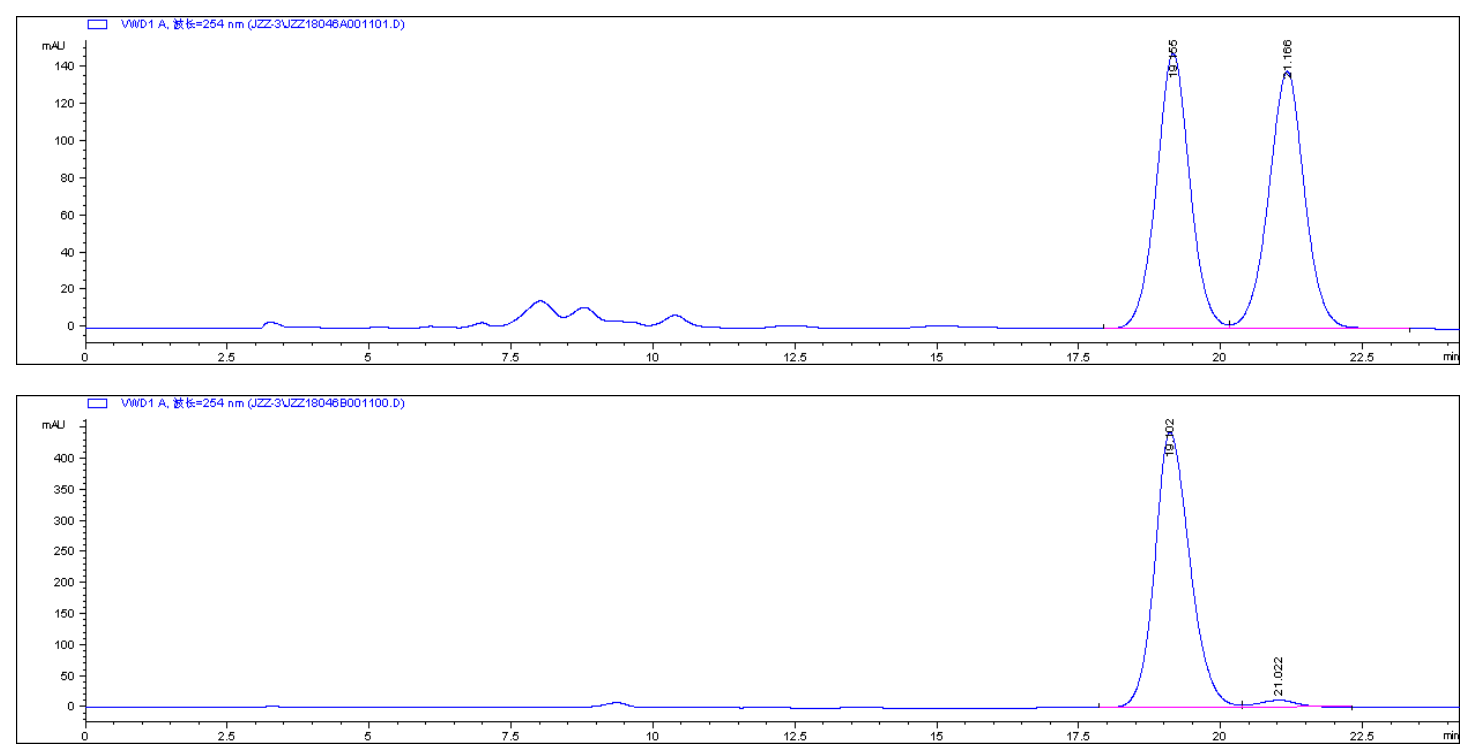
Trifluoromethyl ketone $2 \mathrm{~d}\left(20.9 \mathrm{mg}, 0.1 \mathrm{mmol}, 1.0\right.$ equiv), $\mathrm{Na}_{3} \mathrm{PO}_{4}$. 3d: $\mathrm{Ar}=\mathrm{Ph} \quad 0.1$ equiv) were dissolved in tert-butylbenzene $(1.0 \mathrm{~mL})$ and stirred at $\mathrm{R}=4-\mathrm{CIPh} \quad-10{ }^{\circ} \mathrm{C}$ for $10 \mathrm{~min}$. Then allyl ketone 1a $(43.9 \mathrm{mg}, 0.3 \mathrm{mmol}, 3.0$ equiv) was added. The reaction mixture was stirred at $-10{ }^{\circ} \mathrm{C}$ and monitored by TLC. Upon complete consumption of trifluoromethyl ketone $\mathbf{2 d}$, the reaction mixture was directly loaded onto a short silica gel column, followed by gradient elution with PE/EA mixture (20/1-5/1 ratio). Removing the solvent in vacuo, afforded product 3d. White solid, Mp $110.4-111.6{ }^{\circ} \mathrm{C}$; $33.3 \mathrm{mg}(0.1 \mathrm{mmol}), 94 \%$ yield; $95 \%$ ee; $[\alpha]_{\mathrm{D}}^{26}-46.6$ (c 3.41, $\left.\mathrm{CHCl}_{3}\right) ;{ }^{1} \mathrm{H} \mathrm{NMR}(300 \mathrm{MHz}$, $\left.\mathrm{CDCl}_{3}\right) \delta 7.76(\mathrm{~d}, J=7.32 \mathrm{~Hz}, 2 \mathrm{H}), 7.53-7.50(\mathrm{~m}, 3 \mathrm{H}), 7.44-7.37(\mathrm{~m}, 4 \mathrm{H}), 6.91(\mathrm{~d}, J=15.51$ $\mathrm{Hz}, 1 \mathrm{H}), 6.75-6.65(\mathrm{~m}, 2 \mathrm{H}), 3.51(\mathrm{~s}, 1 \mathrm{H}), 3.21-3.04(\mathrm{~m}, 2 \mathrm{H}) ;{ }^{13} \mathrm{C} \mathrm{NMR}\left(75 \mathrm{MHz}, \mathrm{CDCl}_{3}\right) \delta$ $190.3,140.1,137.0,135.0,134.5,133.2,130.8,128.8,128.6$ (two peaks), 127.9, 125.0 (q, $J=$ $284.3 \mathrm{~Hz}), 76.5$ (q, $J=28.5 \mathrm{~Hz}$ ), 38.9; ${ }^{19} \mathrm{~F}$ NMR $\left(376 \mathrm{MHz}, \mathrm{CDCl}_{3}\right) \delta-79.66$; HRMS (ESI) $\mathrm{m} / \mathrm{z} 377.0531\left(\mathrm{M}+\mathrm{Na}^{+}\right)$, calc. for $\mathrm{C}_{18} \mathrm{H}_{14} \mathrm{ClF}_{3} \mathrm{O}_{2} \mathrm{Na} 377.0532$.

The ee was determined by HPLC analysis. CHIRALPAK IB (4.6 mm i.d. x $250 \mathrm{~mm}$ ); Hexane/2-propanol $=90 / 10$; flow rate $1.0 \mathrm{~mL} / \mathrm{min} ; 25{ }^{\circ} \mathrm{C} ; 254 \mathrm{~nm}$; retention time: $11.4 \mathrm{~min}$ (minor) and 16.3 min (major).
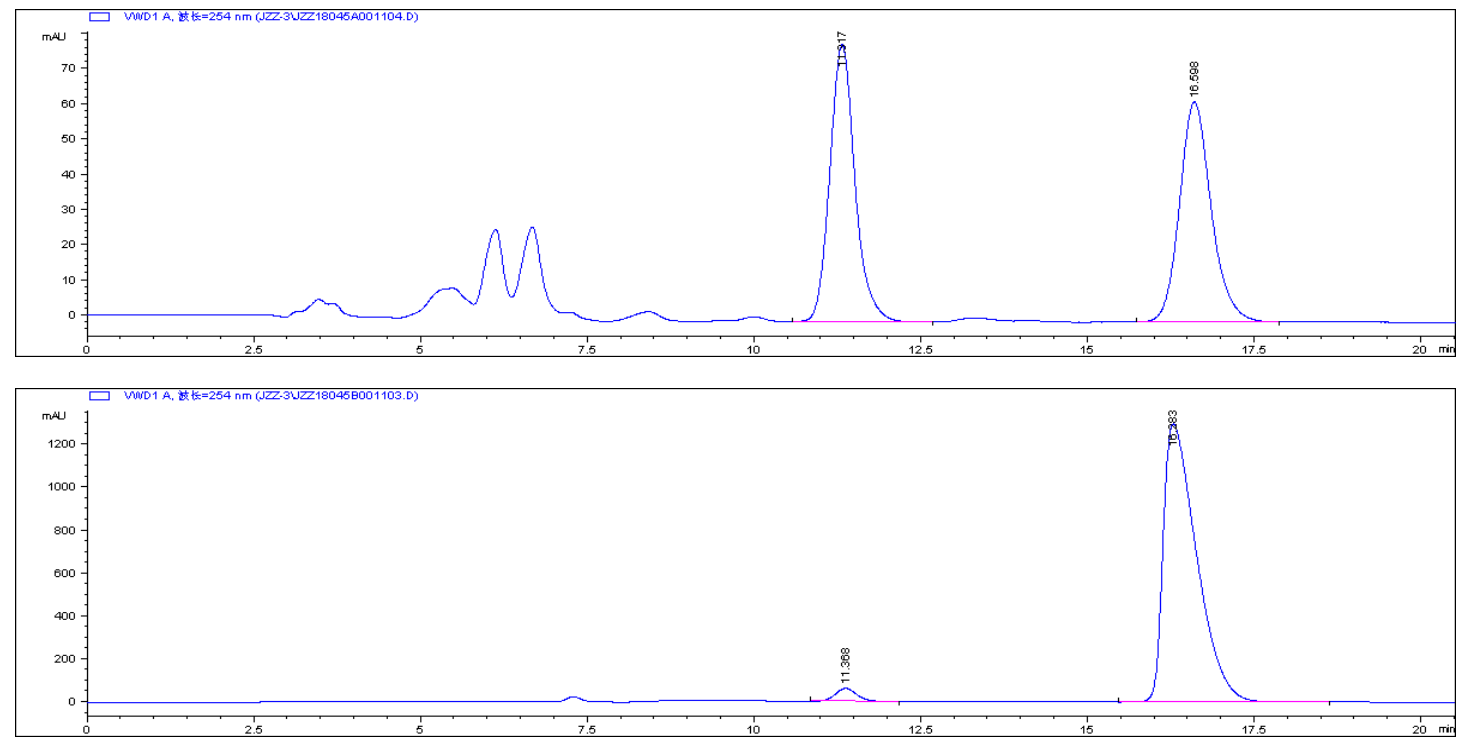

管 3e: $\mathrm{Ar}=\mathrm{Ph}$ 0.1 equiv) were dissolved in tert-butylbenzene $(1.0 \mathrm{~mL})$ and stirred at $\mathrm{R}=4-\mathrm{BrPh}$ 
$-10{ }^{\circ} \mathrm{C}$ for $10 \mathrm{~min}$. Then allyl ketone $\mathbf{1 a}(43.9 \mathrm{mg}, 0.3 \mathrm{mmol}, 3.0$ equiv) was added. The reaction mixture was stirred at $-10{ }^{\circ} \mathrm{C}$ and monitored by TLC. Upon complete consumption of trifluoromethyl ketone $\mathbf{2 e}$, the reaction mixture was directly loaded onto a short silica gel column, followed by gradient elution with PE/EA mixture (20/1-5/1 ratio). Removing the solvent in vacuo, afforded product 3e. White solid, Mp 114.2-115.3 ${ }^{\circ} \mathrm{C} ; 35.9 \mathrm{mg}(0.1 \mathrm{mmol})$; 90\% yield; 94\% ee; $[\alpha]_{\mathrm{D}}^{26}-54.5\left(c 1.42, \mathrm{CHCl}_{3}\right) ;{ }^{1} \mathrm{H}$ NMR $\left(300 \mathrm{MHz}, \mathrm{CDCl}_{3}\right) \delta 7.75(\mathrm{~d}, J=$ $7.4 \mathrm{~Hz}, 2 \mathrm{H}), 7.56-7.53(\mathrm{~m}, 3 \mathrm{H}), 7.47-7.39(\mathrm{~m}, 4 \mathrm{H}), 6.90(\mathrm{~d}, J=15.5 \mathrm{~Hz}, 1 \mathrm{H}), 6.74-6.65$ (m, 1H), $3.50(\mathrm{~s}, 1 \mathrm{H}), 3.20-3.03(\mathrm{~m}, 2 \mathrm{H}) ;{ }^{13} \mathrm{C} \mathrm{NMR}\left(75 \mathrm{MHz}, \mathrm{CDCl}_{3}\right) \delta 190.3,140.1,137.0$, 135.1, 133.2, 131.7, 130.8, 128.6 (two peaks), 128.2 (two peaks), 126.8, 124.9 (q, $J=284.2$ $\mathrm{Hz}), 123.3,76.5$ (q, $J=28.4 \mathrm{~Hz}), 38.9 ;{ }^{19} \mathrm{~F}$ NMR (376 MHz, $\left.\mathrm{CDCl}_{3}\right) \delta-79.64$; HRMS (ESI) $\mathrm{m} / \mathrm{z} 423.0009\left(\mathrm{M}+\mathrm{Na}^{+}\right)$, calc. for $\mathrm{C}_{18} \mathrm{H}_{14} \mathrm{BrF}_{3} \mathrm{O}_{2} \mathrm{Na} 423.0006$.

The ee was determined by HPLC analysis. CHIRALPAK IB (4.6 mm i.d. x $250 \mathrm{~mm}$ ); Hexane/2-propanol $=95 / 05$; flow rate $1.0 \mathrm{~mL} / \mathrm{min} ; 25{ }^{\circ} \mathrm{C} ; 254 \mathrm{~nm}$; retention time: $7.1 \mathrm{~min}$ (minor) and 12.7 min(major).
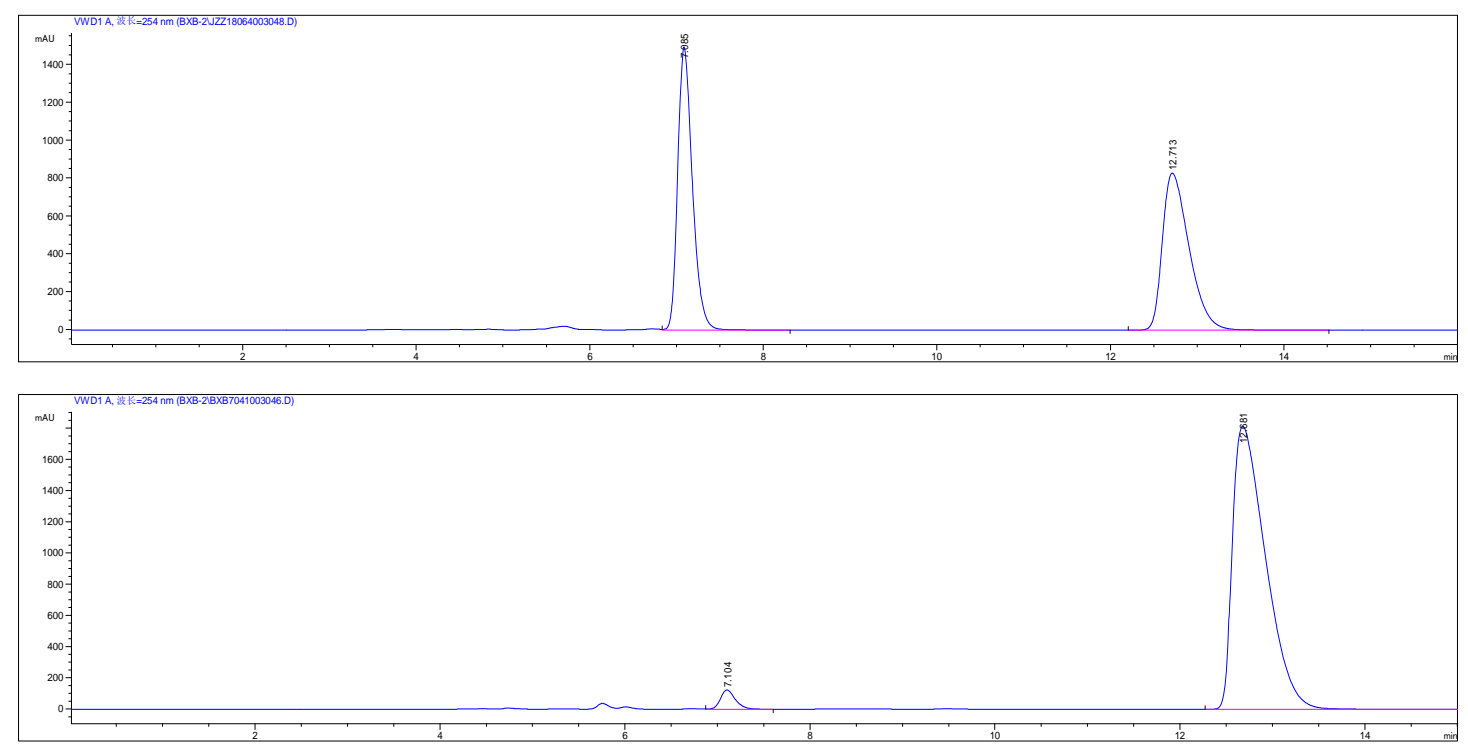

Trifluoromethyl ketone $2 \mathrm{f}$ (19.2 mg, $0.1 \mathrm{mmol}, 1.0$ equiv), $\mathrm{Na}_{3} \mathrm{PO}_{4}$. 3f: $\mathrm{Ar}=\mathrm{Ph} \quad 0.1$ equiv) were dissolved in tert-butylbenzene $(1.0 \mathrm{~mL})$ and stirred at $\mathrm{R}=3-\mathrm{FPh} \quad-10{ }^{\circ} \mathrm{C}$ for $10 \mathrm{~min}$. Then allyl ketone $\mathbf{1 a}(43.9 \mathrm{mg}, 0.3 \mathrm{mmol}, 3.0$ equiv) was added. The reaction mixture was stirred at $-10{ }^{\circ} \mathrm{C}$ and monitored by TLC. Upon complete consumption of trifluoromethyl ketone $\mathbf{2 f}$, the reaction mixture was directly loaded 
onto a short silica gel column, followed by gradient elution with PE/EA mixture (20/1-5/1 ratio). Removing the solvent in vacuo, afforded product 3 f. Colorless oil; $30.1 \mathrm{mg}(0.1 \mathrm{mmol})$, $89 \%$ yield; $91 \% e e ;[\alpha]_{\mathrm{D}}^{26}-41.5\left(\right.$ c $\left.2.56, \mathrm{CHCl}_{3}\right) ;{ }^{1} \mathrm{H} \mathrm{NMR}\left(300 \mathrm{MHz}, \mathrm{CDCl}_{3}\right) \delta 7.78(\mathrm{~d}, J=$ $7.3 \mathrm{~Hz}, 2 \mathrm{H}), 7.55$ (t, $J=7.4 \mathrm{~Hz}, 2 \mathrm{H}), 7.45-7.32(\mathrm{~m}, 5 \mathrm{H}), 7.08$ (t, $J=7.4 \mathrm{~Hz}, 1 \mathrm{H}), 6.93$ (d, $J=$ $15.5 \mathrm{~Hz}, 1 \mathrm{H}), 6.75-6.65(\mathrm{~m}, 1 \mathrm{H}), 3.25(\mathrm{~s}, 1 \mathrm{H}), 3.21-3.04(\mathrm{~m}, 2 \mathrm{H}) ;{ }^{13} \mathrm{C}$ NMR $(75 \mathrm{MHz}$, $\left.\mathrm{CDCl}_{3}\right) \delta 190.3$ (two peaks), 164.5, 161.2, 140.1, 140.0, 138.6, 138.5, 137.0, 133.2, 130.8, 130.2, 130.1, 128.6 (two peaks), 125.0 (q, $J=284.0 \mathrm{~Hz}$ ), 122.0 (two peaks), 116.0, 115.7, 114.2, 113.8 (two peaks), 76.2 (two peaks), 75.8 (two peaks), 39.0; ${ }^{19} \mathrm{~F}$ NMR (376 MHz, $\left.\mathrm{CDCl}_{3}\right) \delta-79.51,-111.68$; HRMS (ESI) $\mathrm{m} / \mathrm{z} 339.1004\left(\mathrm{M}+\mathrm{H}^{+}\right)$, calc. for $\mathrm{C}_{18} \mathrm{H}_{15} \mathrm{~F}_{4} \mathrm{O}_{2}$ 339.1008 .

The ee was determined by HPLC analysis. CHIRALPAK IB-3 (4.6 mm i.d. x $250 \mathrm{~mm}$ ); Hexane/2-propanol $=90 / 10$; flow rate $1.0 \mathrm{~mL} / \mathrm{min} ; 25{ }^{\circ} \mathrm{C} ; 254 \mathrm{~nm}$; retention time: $10.1 \mathrm{~min}$ (major) and $11.5 \mathrm{~min}$ (minor).
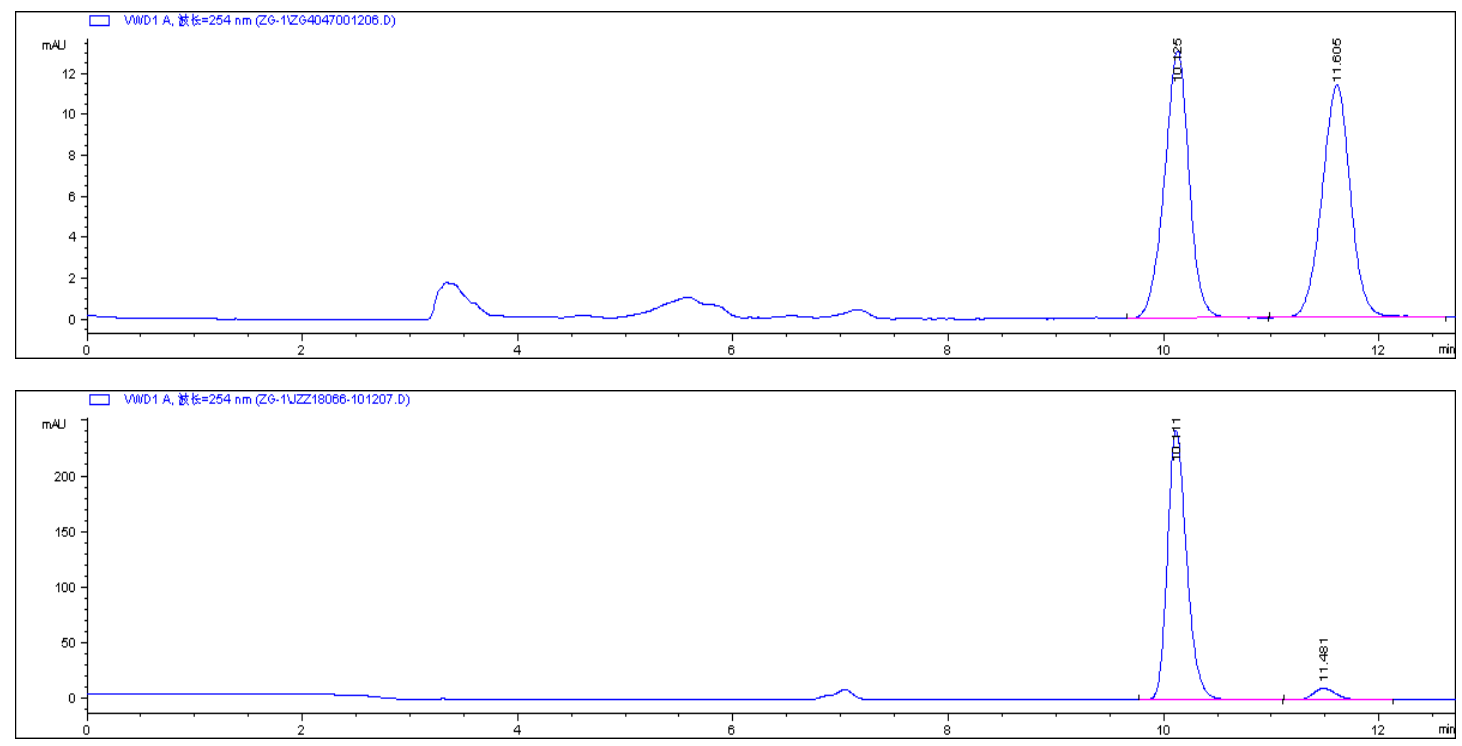

Trifluoromethyl ketone $2 \mathrm{~g}\left(20.9 \mathrm{mg}, 0.1 \mathrm{mmol}, 1.0\right.$ equiv), $\mathrm{Na}_{3} \mathrm{PO}_{4}$.
$\mathrm{CF}_{3}$ 3g: $\mathrm{Ar}=\mathrm{Ph}$ 0.1 equiv) were dissolved in tert-butylbenzene $(1.0 \mathrm{~mL})$ and stirred at $\mathrm{R}=3-\mathrm{ClPh} \quad-10{ }^{\circ} \mathrm{C}$ for $10 \mathrm{~min}$. Then allyl ketone 1a $(43.9 \mathrm{mg}, 0.3 \mathrm{mmol}, 3.0$ equiv) was added. The reaction mixture was stirred at $-10{ }^{\circ} \mathrm{C}$ and monitored by TLC. Upon complete consumption of trifluoromethyl ketone $\mathbf{2 g}$, the reaction mixture was directly loaded onto a short silica gel column, followed by gradient elution with PE/EA mixture (20/1-5/1 
ratio). Removing the solvent in vacuo, afforded product $3 g$. Colorless oil; $33.3 \mathrm{mg}(0.1 \mathrm{mmol})$, 94\% yield; 90\% ee; $[\alpha]_{\mathrm{D}}^{26}-35.6\left(c 3.04, \mathrm{CHCl}_{3}\right) ;{ }^{1} \mathrm{H} 44 \mathrm{NMR}\left(300 \mathrm{MHz}, \mathrm{CDCl}_{3}\right) \delta 7.77(\mathrm{~d}, J=$ $7.3 \mathrm{~Hz}, 2 \mathrm{H}), 7.62(\mathrm{~s}, 1 \mathrm{H}), 7.55(\mathrm{t}, J=7.4 \mathrm{~Hz}, 1 \mathrm{H}), 7.47-7.32(\mathrm{~m}, 5 \mathrm{H}), 6.92(\mathrm{~d}, J=15.5 \mathrm{~Hz}$, $1 \mathrm{H}), 6.74-6.64(\mathrm{~m}, 1 \mathrm{H}), 3.54(\mathrm{~s}, 1 \mathrm{H}), 3.21-3.05(\mathrm{~m}, 2 \mathrm{H}) ;{ }^{13} \mathrm{C} \mathrm{NMR}\left(75 \mathrm{MHz}, \mathrm{CDCl}_{3}\right) \delta 190.4$, $140.0,138.1,137.0,134.7,133.2,130.9,129.8,129.1,128.7,128.6,126.9,126.8,124.9$ (q, $J$ $=284.1 \mathrm{~Hz}$ ), 124.6 (two peaks), 76.4 (q, $J=28.4 \mathrm{~Hz}), 39.0 ;{ }^{19} \mathrm{~F} \mathrm{NMR}\left(376 \mathrm{MHz}, \mathrm{CDCl}_{3}\right) \delta$ -79.48; HRMS (ESI) m/z $377.0533\left(\mathrm{M}+\mathrm{Na}^{+}\right)$, calc. for $\mathrm{C}_{18} \mathrm{H}_{14} \mathrm{ClF}_{3} \mathrm{O}_{2} 377.0532$.

The ee was determined by HPLC analysis. CHIRALPAK IB (4.6 mm i.d. x $250 \mathrm{~mm}$ ); Hexane/2-propanol $=90 / 10$; flow rate $1.0 \mathrm{~mL} / \mathrm{min} ; 25{ }^{\circ} \mathrm{C} ; 254 \mathrm{~nm}$; retention time: $11.6 \mathrm{~min}$ (minor) and 12.6 min (major).
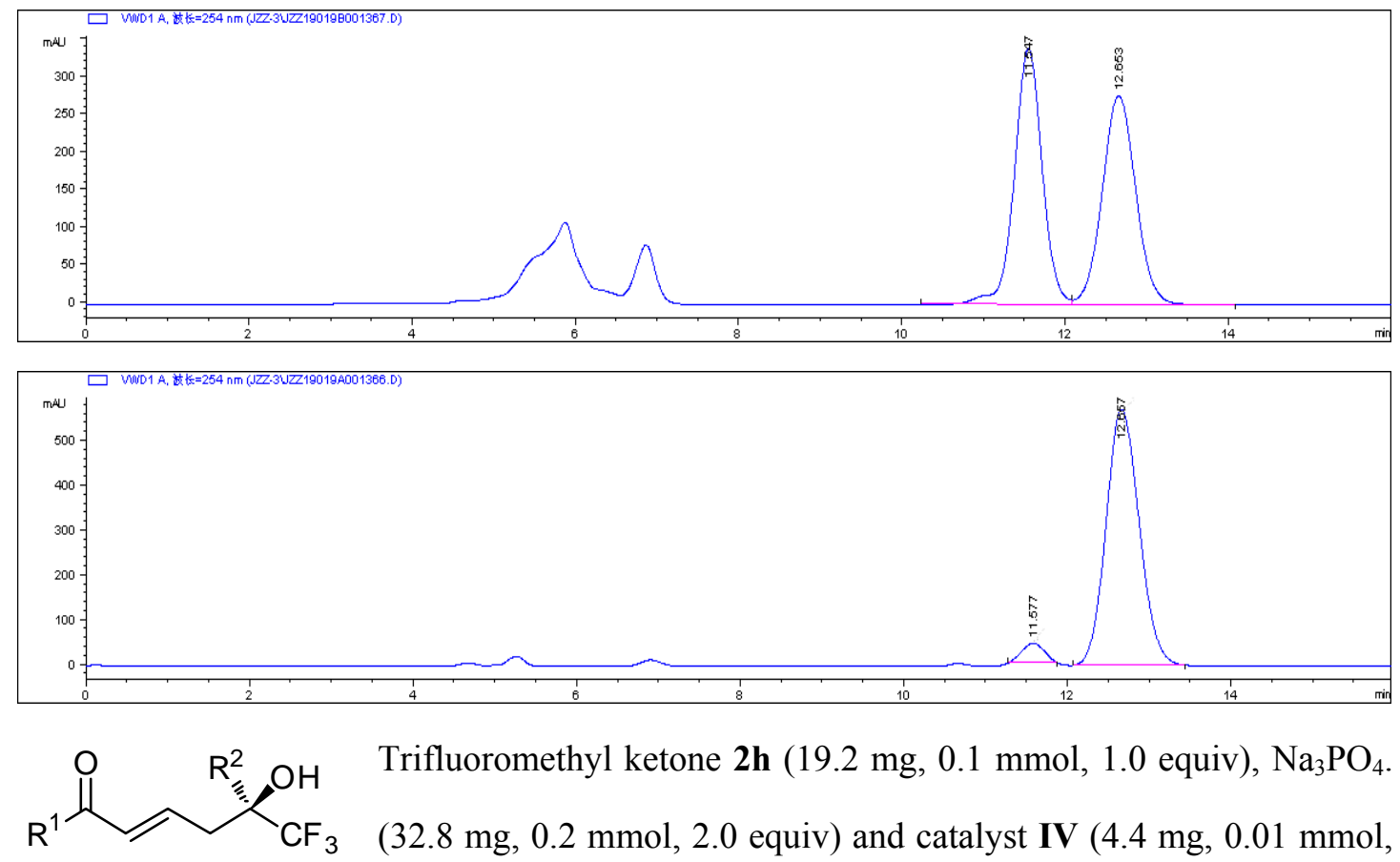
3h: $\mathrm{Ar}=\mathrm{Ph} \quad 0.1$ equiv) were dissolved in tert-butylbenzene $(1.0 \mathrm{~mL})$ and stirred $\mathrm{R}=2-\mathrm{FPh} \quad$ at $-10{ }^{\circ} \mathrm{C}$ for $10 \mathrm{~min}$. Then allyl ketone $1 \mathrm{a}(43.9 \mathrm{mg}, 0.3 \mathrm{mmol}, 3.0$ equiv) was added. The reaction mixture was stirred at $-10{ }^{\circ} \mathrm{C}$ and monitored by TLC. Upon complete consumption of trifluoromethyl ketone $\mathbf{2 h}$, the reaction mixture was directly loaded onto a short silica gel column, followed by gradient elution with PE/EA mixture (20/1-5/1 ratio). Removing the solvent in vacuo, afforded product $\mathbf{3 h}$. White solid, Mp 106.9-107.2 ${ }^{\circ} \mathrm{C}$; $24.7 \mathrm{mg}(0.1 \mathrm{mmol}), 73 \%$ yield; $98 \% e e ;[\alpha]_{\mathrm{D}}^{26}-26.5$ (c 2.25, $\left.\mathrm{CHCl}_{3}\right) ;{ }^{1} \mathrm{H}$ NMR (300 MHz, $\left.\mathrm{CDCl}_{3}\right) \delta 7.77-7.74(\mathrm{~m}, 2 \mathrm{H}), 7.70-7.64(\mathrm{~m}, 1 \mathrm{H}), 7.53(\mathrm{t}, J=7.4 \mathrm{~Hz}, 1 \mathrm{H}), 7.42-7.34(\mathrm{~m}, 3 \mathrm{H})$, 
$7.21(\mathrm{td}, J=7.9,1.1 \mathrm{~Hz}, 1 \mathrm{H}), 7.13-7.06(\mathrm{~m}, 1 \mathrm{H}), 7.00(\mathrm{~d}, J=15.5 \mathrm{~Hz} 1 \mathrm{H}), 6.85-6.75(\mathrm{~m}$, $1 \mathrm{H}), 3.76(\mathrm{~d}, J=5.0 \mathrm{~Hz}, 1 \mathrm{H}), 3.60-3.52(\mathrm{~m}, 1 \mathrm{H}), 3.08-3.00(\mathrm{~m}, 1 \mathrm{H}) ;{ }^{13} \mathrm{C}$ NMR $(75 \mathrm{MHz}$, $\left.\mathrm{CDCl}_{3}\right) \delta 190.5,161.7,158.4,141.0,137.2,133.0,131.4,131.3,130.4,130$ (two peaks), 128.6, 128.5, 124.9 (qd, $J=284.4,1.7 \mathrm{~Hz}$ ), 124.5 (two peaks), 122.4, 122.3, 116.8, 116.4, $76.2(\mathrm{qd}, J=30,3.6 \mathrm{~Hz}), 37.5,37.4 ;{ }^{19} \mathrm{~F}$ NMR $\left(376 \mathrm{MHz}, \mathrm{CDCl}_{3}\right) \delta-80.65,-80.69,-111.46$, -111.50; HRMS (ESI) m/z 339.1004 (M+H'), calc. for $\mathrm{C}_{18} \mathrm{H}_{15} \mathrm{~F}_{4} \mathrm{O}_{2} 339.1008$.

The ee was determined by HPLC analysis. CHIRALPAK IA (4.6 mm i.d. x $250 \mathrm{~mm}$ ); Hexane $/ 2$-propanol $=95 / 05$; flow rate $1.0 \mathrm{~mL} / \mathrm{min} ; 25{ }^{\circ} \mathrm{C} ; 254 \mathrm{~nm}$; retention time: $9.1 \mathrm{~min}$ (major) and $11.3 \mathrm{~min}$ (minor).
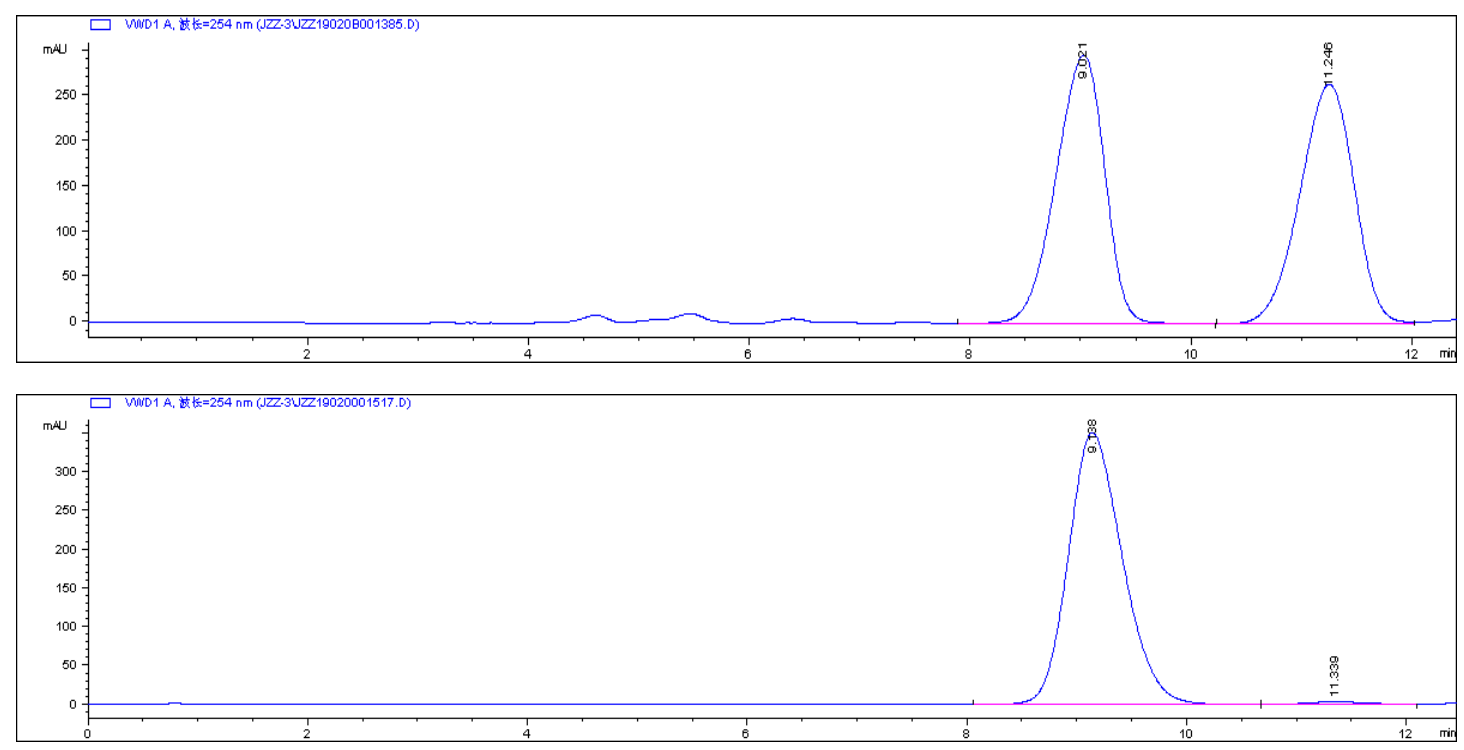

Trifluoromethyl ketone $2 \mathrm{i}\left(18.8 \mathrm{mg}, 0.1 \mathrm{mmol}, 1.0\right.$ equiv), $\mathrm{Na}_{3} \mathrm{PO}_{4}$.
$\mathrm{CF}_{3}$ 3i: $\mathrm{Ar}=\mathrm{Ph}$ 0.1 equiv) were dissolved in tert-butylbenzene $(1.0 \mathrm{~mL})$ and stirred at $\mathrm{R}=4-\mathrm{MePh} \quad-10{ }^{\circ} \mathrm{C}$ for $10 \mathrm{~min}$. Then allyl ketone 1a $(43.9 \mathrm{mg}, 0.3 \mathrm{mmol}, 3.0$ equiv) was added. The reaction mixture was stirred at $-10{ }^{\circ} \mathrm{C}$ and monitored by TLC. Upon complete consumption of trifluoromethyl ketone $\mathbf{2} \mathbf{i}$, the reaction mixture was directly loaded onto a short silica gel column, followed by gradient elution with PE/EA mixture (20/1-5/1 ratio). Removing the solvent in vacuo, afforded product 3i. White solid, Mp 109.2-110.6 ${ }^{\circ} \mathrm{C}$; $25.1 \mathrm{mg}(0.1 \mathrm{mmol}), 75 \%$ yield; $94 \% e e ;[\alpha]_{\mathrm{D}}^{26}-51.2\left(c\right.$ 1.32, $\left.\mathrm{CHCl}_{3}\right) ;{ }^{1} \mathrm{H}$ NMR $(300 \mathrm{MHz}$, $\left.\mathrm{CDCl}_{3}\right) \delta 7.78-7.75(\mathrm{~m}, 2 \mathrm{H}), 7.54(\mathrm{t}, J=7.4 \mathrm{~Hz}, 1 \mathrm{H}), 7.46-7.38(\mathrm{~m}, 4 \mathrm{H}), 7.22(\mathrm{~d}, J=8.1 \mathrm{~Hz}$, 2H), $6.91(\mathrm{~d}, J=15.5 \mathrm{~Hz}, 1 \mathrm{H}), 6.76-6.66(\mathrm{~m}, 1 \mathrm{H}), 3.23-3.02(\mathrm{~m}, 2 \mathrm{H}), 2.97(\mathrm{~d}, J=5.9 \mathrm{~Hz}$, 
1H), 2.37 (s, 3H); ${ }^{13} \mathrm{C}$ NMR (75 MHz, $\left.\mathrm{CDCl}_{3}\right) \delta$ 190.3, 140.6, 138.8, 137.2, 133.0, 132.9, 130.7, 129.3, 128.6, 128.5, 127.1, 125.2 (q, $J=284.2 \mathrm{~Hz}), 76.7$ (q, $J=28.2 \mathrm{~Hz}), 39.0,21.0$; ${ }^{19} \mathrm{~F}$ NMR $\left(376 \mathrm{MHz}, \mathrm{CDCl}_{3}\right) \delta$-79.74; HRMS (ESI) m/z $357.1077\left(\mathrm{M}+\mathrm{Na}^{+}\right)$, calc. for $\mathrm{C}_{19} \mathrm{H}_{17} \mathrm{~F}_{3} \mathrm{O}_{2} \mathrm{Na} 357.1078$.

The ee was determined by HPLC analysis. CHIRALPAK IB (4.6 mm i.d. x $250 \mathrm{~mm}$ ); Hexane/2-propanol $=90 / 10$; flow rate $1.0 \mathrm{~mL} / \mathrm{min} ; 25^{\circ} \mathrm{C} ; 254 \mathrm{~nm}$; retention time: $10.0 \mathrm{~min}$ (major) and $18.3 \mathrm{~min}$ (minor).
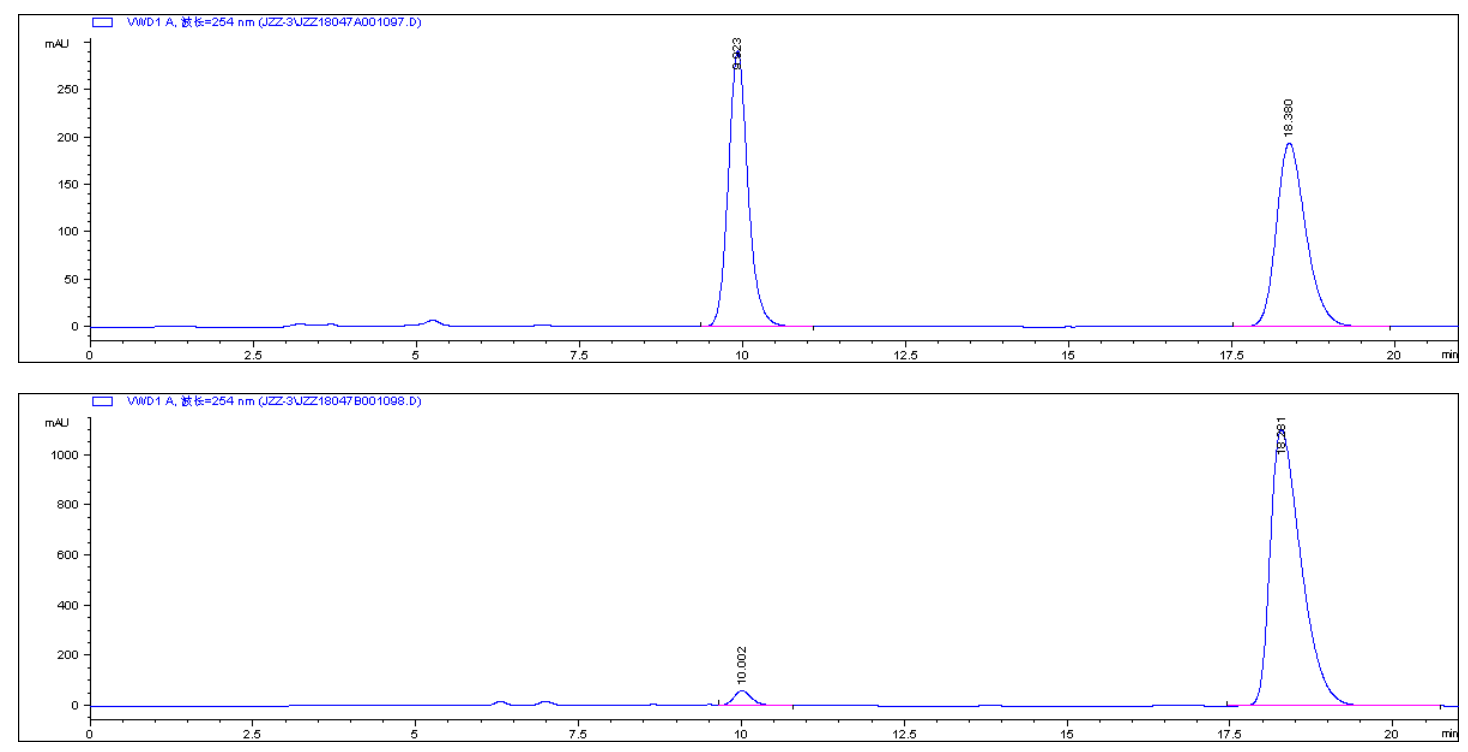

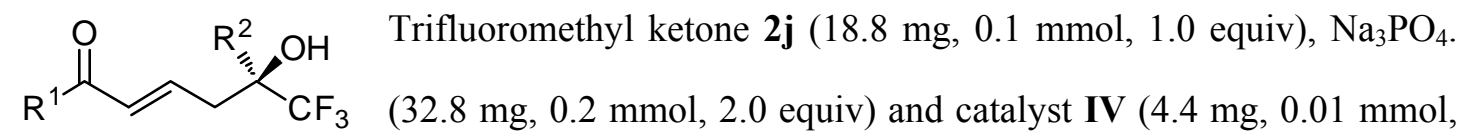
3j: $\mathrm{Ar}=\mathrm{Ph} \quad 0.1$ equiv $)$ were dissolved in tert-butylbenzene $(1.0 \mathrm{~mL})$ and stirred at $\mathrm{R}=3-\mathrm{MePh} \quad-10{ }^{\circ} \mathrm{C}$ for $10 \mathrm{~min}$. Then allyl ketone $\mathbf{1 a}(43.9 \mathrm{mg}, 0.3 \mathrm{mmol}, 3.0$ equiv) was added. The reaction mixture was stirred at $-10{ }^{\circ} \mathrm{C}$ and monitored by TLC. Upon complete consumption of trifluoromethyl ketone $\mathbf{2} \mathbf{j}$, the reaction mixture was directly loaded onto a short silica gel column, followed by gradient elution with PE/EA mixture (20/1-5/1 ratio). Removing the solvent in vacuo, afforded product $\mathbf{3 j}$. White solid, Mp $78.5-80.4{ }^{\circ} \mathrm{C}$; $25.4 \mathrm{mg}(0.1 \mathrm{mmol}), 76 \%$ yield; $94 \% e e ;[\alpha]_{\mathrm{D}}^{26}-45.8\left(c 1.84, \mathrm{CHCl}_{3}\right) ;{ }^{1} \mathrm{H}$ NMR $(300 \mathrm{MHz}$, $\left.\mathrm{CDCl}_{3}\right) \delta 7.78-7.74(\mathrm{~m}, 2 \mathrm{H}), 7.54(\mathrm{t}, J=7.4 \mathrm{~Hz}, 1 \mathrm{H}), 7.43-7.28(\mathrm{~m}, 5 \mathrm{H}), 7.19(\mathrm{~d}, J=7.2 \mathrm{~Hz}$, 1H), $6.92(\mathrm{~d}, J=15.5 \mathrm{~Hz}, 1 \mathrm{H}), 6.76-6.66(\mathrm{~m}, 1 \mathrm{H}), 3.24-3.06(\mathrm{~m}, 2 \mathrm{H}), 3.02$, (s, 1H) 2.38 (s, $3 \mathrm{H}) ;{ }^{13} \mathrm{C} \mathrm{NMR}\left(75 \mathrm{MHz}, \mathrm{CDCl}_{3}\right) \delta 190.4,140.7,138.3,137.2,135.8,133.0,130.8,129.7$, 128.7, 128.6, 128.5, 127.0 (two peaks), 125.2 (q, $J=284.0 \mathrm{~Hz}$ ), 123.4 (two peaks), 77.7 (q, $J$ 
$=28.2 \mathrm{~Hz}$ ), 39.1, 21.6; ${ }^{19} \mathrm{~F}$ NMR (376 MHz, $\left.\mathrm{CDCl}_{3}\right) \delta-79.99$; HRMS (ESI) m/z 357.1087 $\left(\mathrm{M}+\mathrm{Na}^{+}\right)$, calc. for $\mathrm{C}_{19} \mathrm{H}_{17} \mathrm{~F}_{3} \mathrm{O}_{2} \mathrm{Na} 357.1078$.

The ee was determined by HPLC analysis. CHIRALPAK IB (4.6 mm i.d. x $250 \mathrm{~mm}$ ); Hexane/2-propanol $=90 / 10$; flow rate $1.0 \mathrm{~mL} / \mathrm{min} ; 25{ }^{\circ} \mathrm{C} ; 254 \mathrm{~nm}$; retention time: $9.6 \mathrm{~min}$ (minor) and 10.8 min (major).
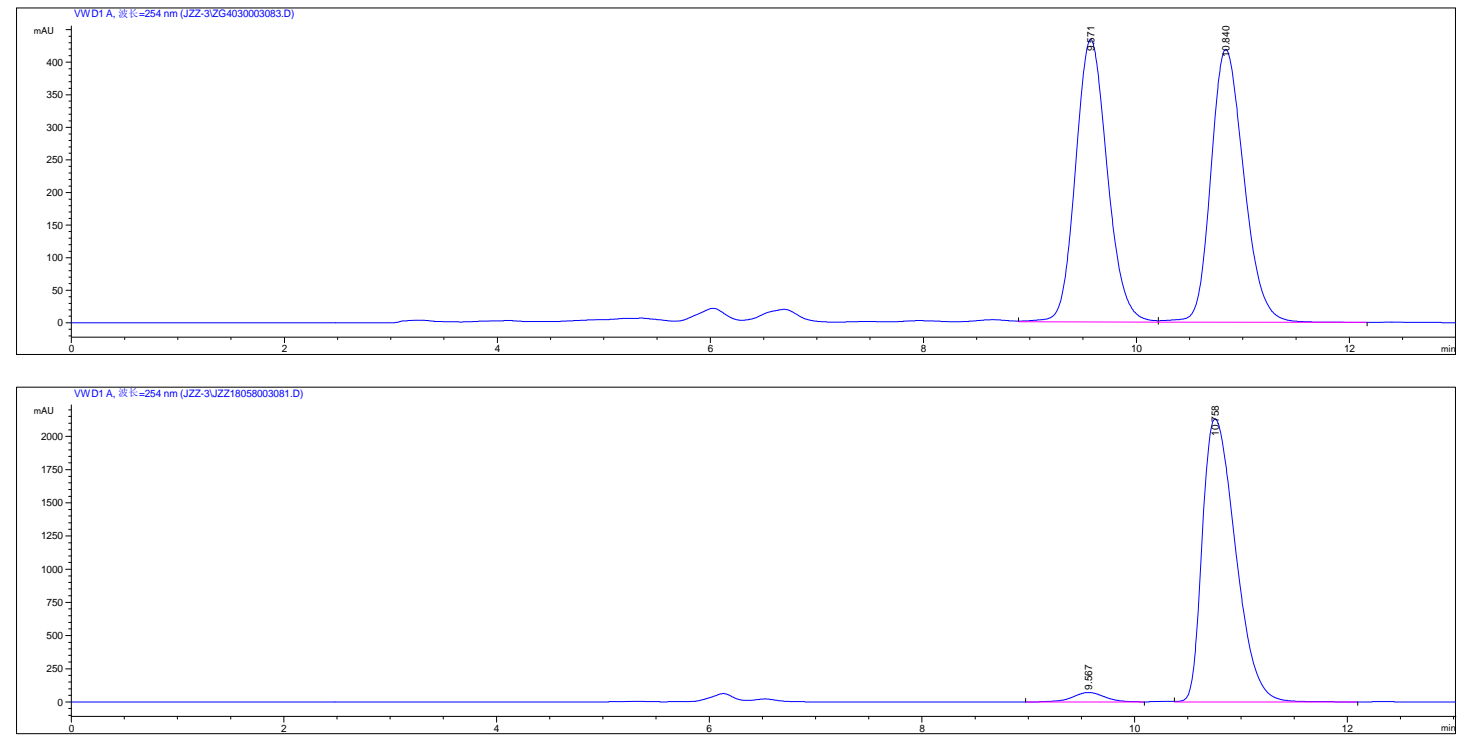

少 3k: $\mathrm{Ar}=\mathrm{Ph}$ 0.1 equiv) were dissolved in tert-butylbenzene $(1.0 \mathrm{~mL})$ and stirred at $\mathrm{R}=$ 2-naphtyl $\quad-10{ }^{\circ} \mathrm{C}$ for $10 \mathrm{~min}$. Then allyl ketone $1 \mathrm{a}(43.9 \mathrm{mg}, 0.3 \mathrm{mmol}, 3.0$ equiv) was added. The reaction mixture was stirred at $-10{ }^{\circ} \mathrm{C}$ and monitored by TLC. Upon complete consumption of trifluoromethyl ketone $\mathbf{2 k}$, the reaction mixture was directly loaded onto a short silica gel column, followed by gradient elution with PE/EA mixture (20/1-5/1 ratio). Removing the solvent in vacuo, afforded product 3k. White solid, Mp $163.1-164.9^{\circ} \mathrm{C}$; $30.0 \mathrm{mg}(0.1 \mathrm{mmol}), 81 \%$ yield; $94 \% e e ;[\alpha]_{\mathrm{D}}^{26}-40.1$ (c 0.87, $\left.\mathrm{CHCl}_{3}\right) ;{ }^{1} \mathrm{H}$ NMR $(300 \mathrm{MHz}$, acetone- $\left.d_{6}\right) \delta 8.30(\mathrm{~s}, 1 \mathrm{H}), 8.00-7.92(\mathrm{~m}, 3 \mathrm{H}), 7.83(\mathrm{~d}, J=8.8 \mathrm{~Hz}, 1 \mathrm{H}), 7.72(\mathrm{~d}, J=7.9 \mathrm{~Hz}$, 2H), 7.58-7.51 (m, 3H), $7.36(\mathrm{t}, J=7.7 \mathrm{~Hz}, 2 \mathrm{H}), 7.15(\mathrm{~d}, J=15.5 \mathrm{~Hz}, 1 \mathrm{H}), 6.76-6.67(\mathrm{~m}$, $1 \mathrm{H}), 6.13(\mathrm{~s}, 1 \mathrm{H}), 3.63-3.56(\mathrm{~m}, 1 \mathrm{H}), 3.31-3.24(\mathrm{~m}, 1 \mathrm{H}) ;{ }^{13} \mathrm{C}$ NMR $\left(75 \mathrm{MHz}\right.$, acetone- $\left.d_{6}\right) \delta$ $190.1,141.7,138.4,135.2,134.0,133.9,133.6,131.1,129.4,129.3,129.2,128.8,128.4$, $127.8,127.6,127.3,126.9(\mathrm{q}, J=284.2 \mathrm{~Hz}), 77.5(\mathrm{q}, J=27.5 \mathrm{~Hz}), 38.8 ;{ }^{19} \mathrm{~F}$ NMR $(376 \mathrm{MHz}$, $\left.\mathrm{CDCl}_{3}\right) \delta-79.23$; HRMS (ESI) m/z $371.1257\left(\mathrm{M}+\mathrm{H}^{+}\right)$, calc. for $\mathrm{C}_{22} \mathrm{H}_{18} \mathrm{~F}_{3} \mathrm{O}_{2} 371.1259$. 
The ee was determined by HPLC analysis. CHIRALPAK IE (4.6 mm i.d. x $250 \mathrm{~mm}$ ); Hexane/2-propanol $=95 / 05$; flow rate $1.0 \mathrm{~mL} / \mathrm{min} ; 25{ }^{\circ} \mathrm{C} ; 254 \mathrm{~nm}$; retention time: $11.8 \mathrm{~min}$ (minor) and 12.7 min (major).
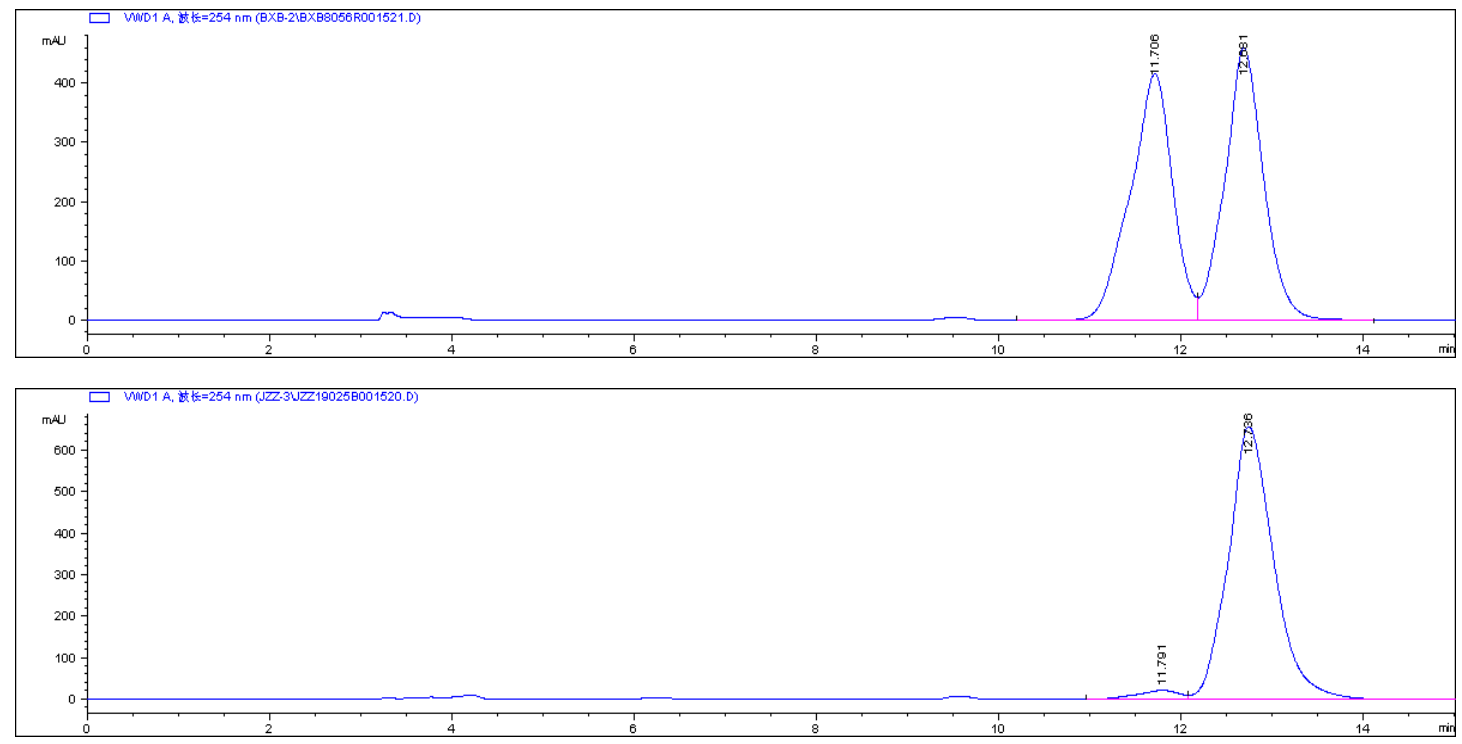

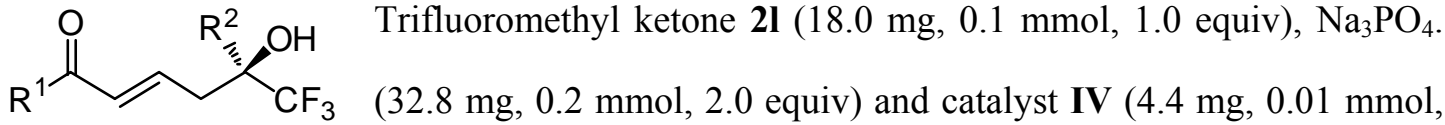
3l: $\mathrm{Ar}=\mathrm{Ph}$ 0.1 equiv) were dissolved in tert-butylbenzene $(1.0 \mathrm{~mL})$ and stirred at $\mathrm{R}=$ 2-thienyl $\quad-10{ }^{\circ} \mathrm{C}$ for $10 \mathrm{~min}$. Then allyl ketone 1a $(43.9 \mathrm{mg}, 0.3 \mathrm{mmol}, 3.0$ equiv) was added. The reaction mixture was stirred at $-10{ }^{\circ} \mathrm{C}$ and monitored by TLC. Upon complete consumption of trifluoromethyl ketone $\mathbf{2 1}$, the reaction mixture was directly loaded onto a short silica gel column, followed by gradient elution with PE/EA mixture (20/1-5/1 ratio). Removing the solvent in vacuo, afforded product 31. White solid, Mp 77.2-82.2 ${ }^{\circ} \mathrm{C}$; $30.3 \mathrm{mg}(0.1 \mathrm{mmol}), 93 \%$ yield; $92 \%$ ee; $[\alpha]_{\mathrm{D}}^{26}-26.6\left(c 2.26, \mathrm{CHCl}_{3}\right) ;{ }^{1} \mathrm{H} \mathrm{NMR}(300 \mathrm{MHz}$, $\left.\mathrm{CDCl}_{3}\right) \delta 7.81(\mathrm{~d}, J=7.3 \mathrm{~Hz}, 2 \mathrm{H}), 7.55(\mathrm{t}, J=7.4 \mathrm{~Hz}, 1 \mathrm{H}), 7.42(\mathrm{t}, J=7.5 \mathrm{~Hz}, 2 \mathrm{H}), 7.36(\mathrm{~d}, J$ $=5.1 \mathrm{~Hz}, 1 \mathrm{H}), 7.16(\mathrm{~d}, J=3.4 \mathrm{~Hz}, 1 \mathrm{H}), 7.04(\mathrm{dd}, J=4.9,3.9 \mathrm{~Hz}, 1 \mathrm{H}), 6.94(\mathrm{~d}, J=15.5 \mathrm{~Hz}$, $1 \mathrm{H}), 6.87-6.78(\mathrm{~m}, 1 \mathrm{H}), 3.58(\mathrm{~s}, 1 \mathrm{H}), 3.13(\mathrm{~s}, 1 \mathrm{H}), 3.10(\mathrm{~s}, 1 \mathrm{H}) ;{ }^{13} \mathrm{C} \mathrm{NMR}\left(75 \mathrm{MHz}, \mathrm{CDCl}_{3}\right)$ $\delta 190.4,140.2,140.0,137.1,133.1,130.8,128.7,128.6,127.3,126.6,126.1,124.6$ (q, $J=$ $283.9 \mathrm{~Hz}), 76.4(\mathrm{q}, J=29.7 \mathrm{~Hz}), 40.0 ;{ }^{19} \mathrm{~F}$ NMR $\left(376 \mathrm{MHz}, \mathrm{CDCl}_{3}\right) \delta-80.58$; HRMS (ESI) $\mathrm{m} / \mathrm{z} 349.0493\left(\mathrm{M}+\mathrm{Na}^{+}\right)$, calc. for $\mathrm{C}_{16} \mathrm{H}_{13} \mathrm{~F}_{3} \mathrm{O}_{2} \mathrm{SNa} 349.0486$.

The ee was determined by HPLC analysis. CHIRALPAK IB-3 (4.6 mm i.d. x $250 \mathrm{~mm}$ ); Hexane/2-propanol $=90 / 10$; flow rate $1.0 \mathrm{~mL} / \mathrm{min} ; 25{ }^{\circ} \mathrm{C} ; 254 \mathrm{~nm}$; retention time: $10.3 \mathrm{~min}$ 
(major) and $11.6 \mathrm{~min}$ (minor).
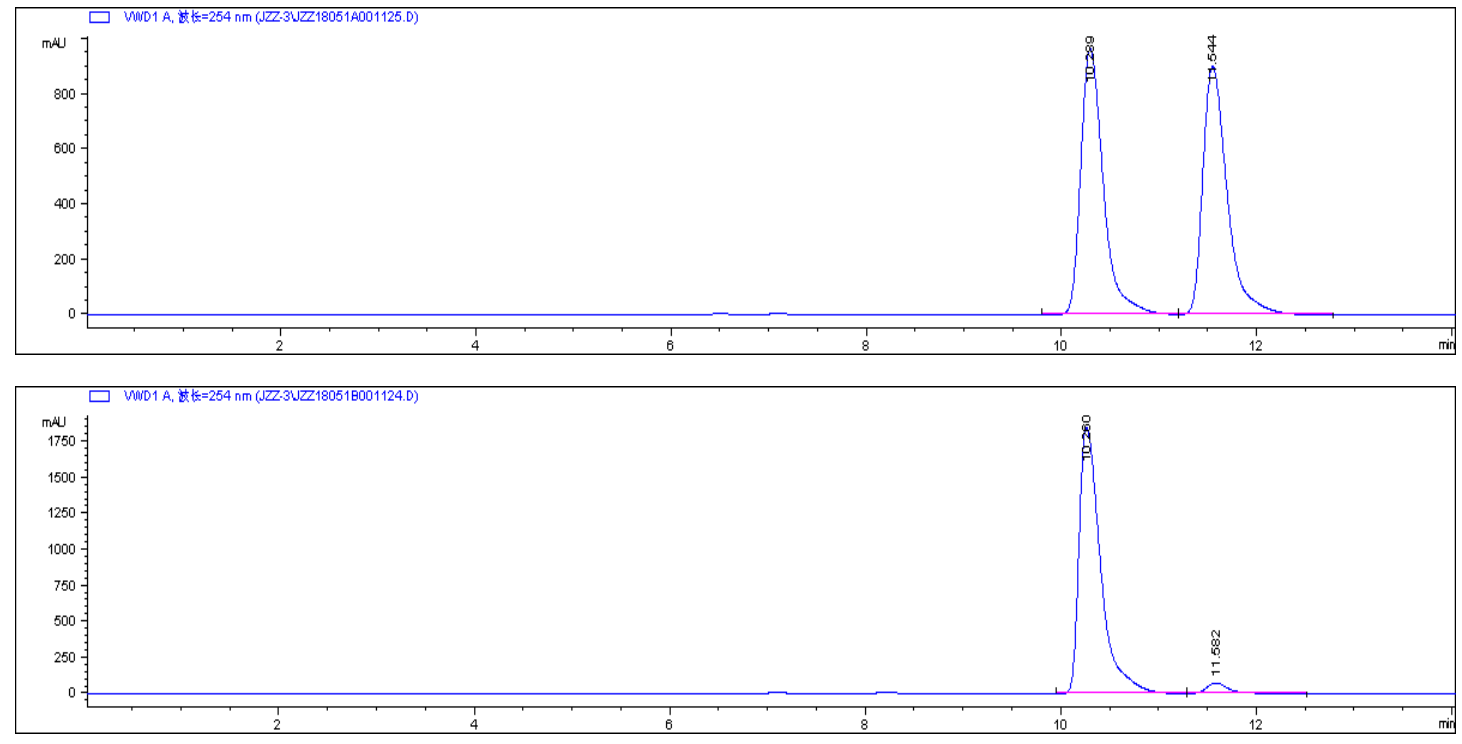

Trifluoromethyl ketone $2 \mathrm{~m}\left(12.6 \mathrm{mg}, 0.1 \mathrm{mmol}, 1.0\right.$ equiv), $\mathrm{Na}_{3} \mathrm{PO}_{4}$.
$\mathrm{CF}_{3}$ $3 \mathrm{~m}: \mathrm{Ar}=\mathrm{Ph} \quad 0.1$ equiv $)$ were dissolved in tert-butylbenzene $(1.0 \mathrm{~mL})$ and stirred at $\mathrm{R}=\mathrm{Et} \quad-10{ }^{\circ} \mathrm{C}$ for $10 \mathrm{~min}$. Then allyl ketone 1a $(43.9 \mathrm{mg}, 0.3 \mathrm{mmol}, 3.0$ equiv) was added. The reaction mixture was stirred at $-10{ }^{\circ} \mathrm{C}$ and monitored by TLC. Upon complete consumption of trifluoromethyl ketone $\mathbf{2 m}$, the reaction mixture was directly loaded onto a short silica gel column, followed by gradient elution with PE/EA mixture (20/1-5/1 ratio). Removing the solvent in vacuo, afforded product $3 \mathbf{m}$. Colorless oil; $19.6 \mathrm{mg}(0.1$ mmol), 72\% yield; 93\% ee; $[\alpha]_{\mathrm{D}}^{26}+12.4\left(c \quad 0.74, \mathrm{CHCl}_{3}\right) ;{ }^{1} \mathrm{H}$ NMR $\left(300 \mathrm{MHz}, \mathrm{CDCl}_{3}\right) \delta$ 7.94-7.92 (m, 2H), 7.58 (t, $J=7.3 \mathrm{~Hz}, 1 \mathrm{H}), 7.48$ (t, $J=7.5 \mathrm{~Hz}, 2 \mathrm{H}), 7.08-6.93$ (m, 2H), 2.72 $(\mathrm{d}, J=5.07 \mathrm{~Hz}, 2 \mathrm{H}), 2.36(\mathrm{~s}, 1 \mathrm{H}), 1.81(\mathrm{q}, J=7.6 \mathrm{~Hz}, 2 \mathrm{H}), 1.04(\mathrm{t}, J=7.6 \mathrm{~Hz}, 3 \mathrm{H}) ;{ }^{13} \mathrm{C} \mathrm{NMR}$ $\left(75 \mathrm{MHz}, \mathrm{CDCl}_{3}\right) \delta 190.2,141.2,137.4,133.0,129.8,128.6$ (two peaks), 126.2 (q, $J=284.9$ $\mathrm{Hz}), 75.4(\mathrm{q}, J=26.9 \mathrm{~Hz}), 36.5,27.0,7.2 ;{ }^{19} \mathrm{~F}$ NMR (376 MHz, $\left.\mathrm{CDCl}_{3}\right) \delta-79.51$; HRMS (ESI) $\mathrm{m} / \mathrm{z} 273.1103\left(\mathrm{M}+\mathrm{H}^{+}\right)$, calc. for $\mathrm{C}_{14} \mathrm{H}_{16} \mathrm{~F}_{3} \mathrm{O}_{2} 273.1102$.

The ee was determined by HPLC analysis. CHIRALPAK IB (4.6 mm i.d. x $250 \mathrm{~mm}$ ); Hexane $/ 2$-propanol $=90 / 10$; flow rate $1.0 \mathrm{~mL} / \mathrm{min} ; 25{ }^{\circ} \mathrm{C} ; 254 \mathrm{~nm}$; retention time: $7.3 \mathrm{~min}$ (major) and $8.2 \mathrm{~min}$ (minor). 

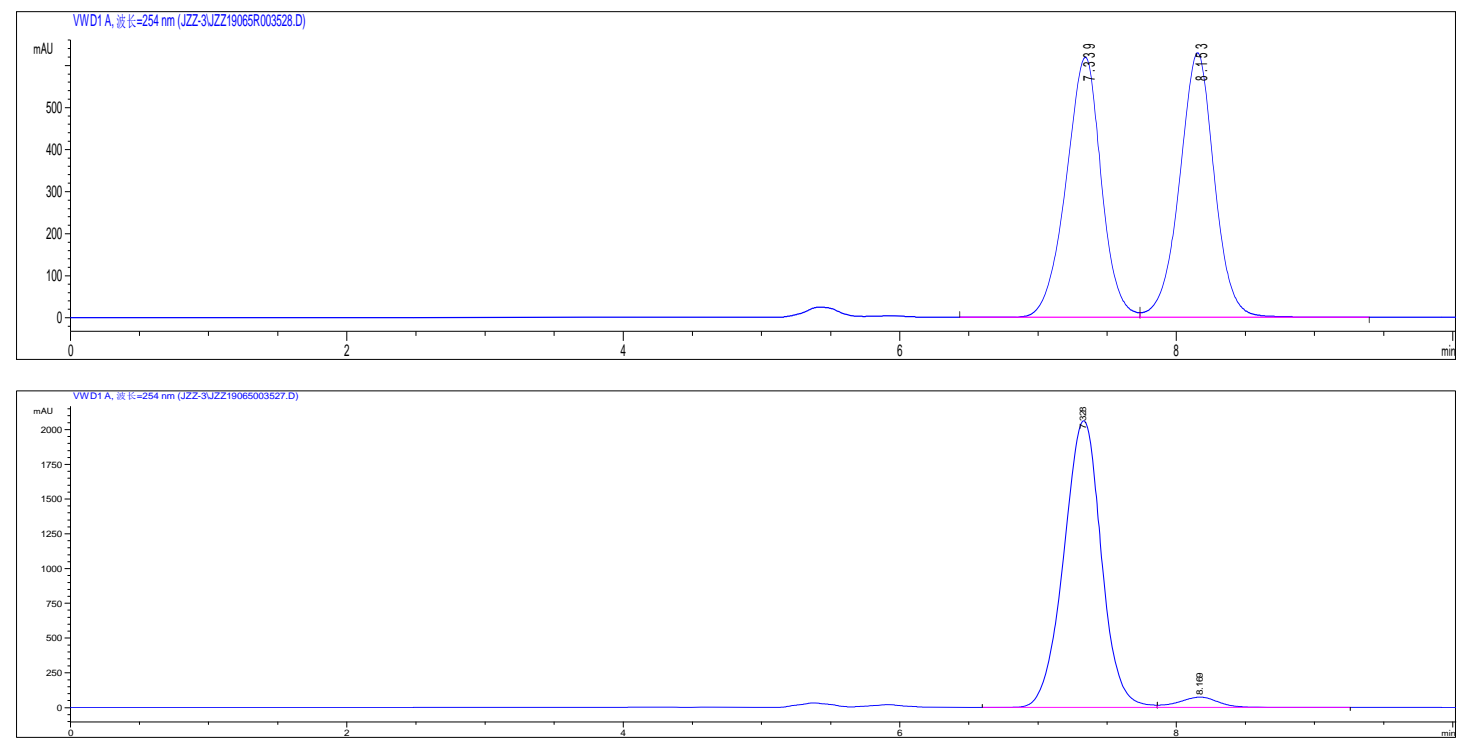

$\underbrace{\text { Trifluoromethyl ketone } 2 \mathrm{a}(17.4 \mathrm{mg}, 0.1 \mathrm{mmol}, 1.0 \mathrm{equiv}), \mathrm{Na}_{3} \mathrm{PO}_{4}}_{\mathrm{CF}_{3}}$ 3n: $\mathrm{Ar}=4-\mathrm{FPh}$ 0.1 equiv) were dissolved in tert-butylbenzene $(1.0 \mathrm{~mL})$ and stirred at $\mathrm{R}=\mathrm{Ph}$ $-10{ }^{\circ} \mathrm{C}$ for $10 \mathrm{~min}$. Then allyl ketone 1 n $(49.3 \mathrm{mg}, 0.3 \mathrm{mmol}, 3.0$ equiv) was added. The reaction mixture was stirred at $-10{ }^{\circ} \mathrm{C}$ and monitored by TLC. Upon complete consumption of trifluoromethyl ketone $\mathbf{2 a}$, the reaction mixture was directly loaded onto a short silica gel column, followed by gradient elution with PE/EA mixture (20/1-5/1 ratio). Removing the solvent in vacuo, afforded product $3 \mathbf{n}$. White solid, $\mathrm{Mp} 106.7-108.0{ }^{\circ} \mathrm{C}$; $31.5 \mathrm{mg}(0.1 \mathrm{mmol}), 93 \%$ yield; $94 \%$ ee; $[\alpha]_{\mathrm{D}}^{26}-30.2\left(c 2.30, \mathrm{CHCl}_{3}\right) ;{ }^{1} \mathrm{H}$ NMR $(300 \mathrm{MHz}$, $\left.\mathrm{CDCl}_{3}\right) \delta 7.79-7.72(\mathrm{~m}, 2 \mathrm{H}), 7.57(\mathrm{~d}, J=7.0 \mathrm{~Hz}, 2 \mathrm{H}), 7.45-7.36(\mathrm{~m}, 3 \mathrm{H}), 7.10-7.03(\mathrm{~m}, 2 \mathrm{H})$, $6.86(\mathrm{~d}, J=15.5 \mathrm{~Hz}, 1 \mathrm{H}), 6.75-6.65(\mathrm{~m}, 1 \mathrm{H}), 3.27(\mathrm{~s}, 1 \mathrm{H}), 3.25-3.17(\mathrm{~m}, 1 \mathrm{H}), 3.12-3.04(\mathrm{~m}$, $1 \mathrm{H}) ;{ }^{13} \mathrm{C}$ NMR $\left(75 \mathrm{MHz}, \mathrm{CDCl}_{3}\right) \delta 188.89,167.4,164.0,140.9,135.9,133.5,133.4,131.3$, $131.2,130.3,128.9,128.6,126.3,125.2(\mathrm{q}, J=284.1 \mathrm{~Hz}), 115.8,115.5,76.7(\mathrm{q}, J=28.1 \mathrm{~Hz})$, 39.0; ${ }^{19} \mathrm{~F}$ NMR $\left(376 \mathrm{MHz}, \mathrm{CDCl}_{3}\right) \delta-79.56,-105.09 ;$ HRMS (ESI) m/z 339.1015 $\left(\mathrm{M}+\mathrm{H}^{+}\right)$, calc. for $\mathrm{C}_{18} \mathrm{H}_{15} \mathrm{~F}_{4} \mathrm{O}_{2} 339.1008$.

The ee was determined by HPLC analysis. CHIRALPAK IB (4.6 mm i.d. x $250 \mathrm{~mm})$; Hexane/2-propanol $=90 / 10$; flow rate $1.0 \mathrm{ml} / \mathrm{min} ; 25{ }^{\circ} \mathrm{C} ; 254 \mathrm{~nm}$; retention time: $8.5 \mathrm{~min}$ (major) and $11.8 \mathrm{~min}$ (minor). 

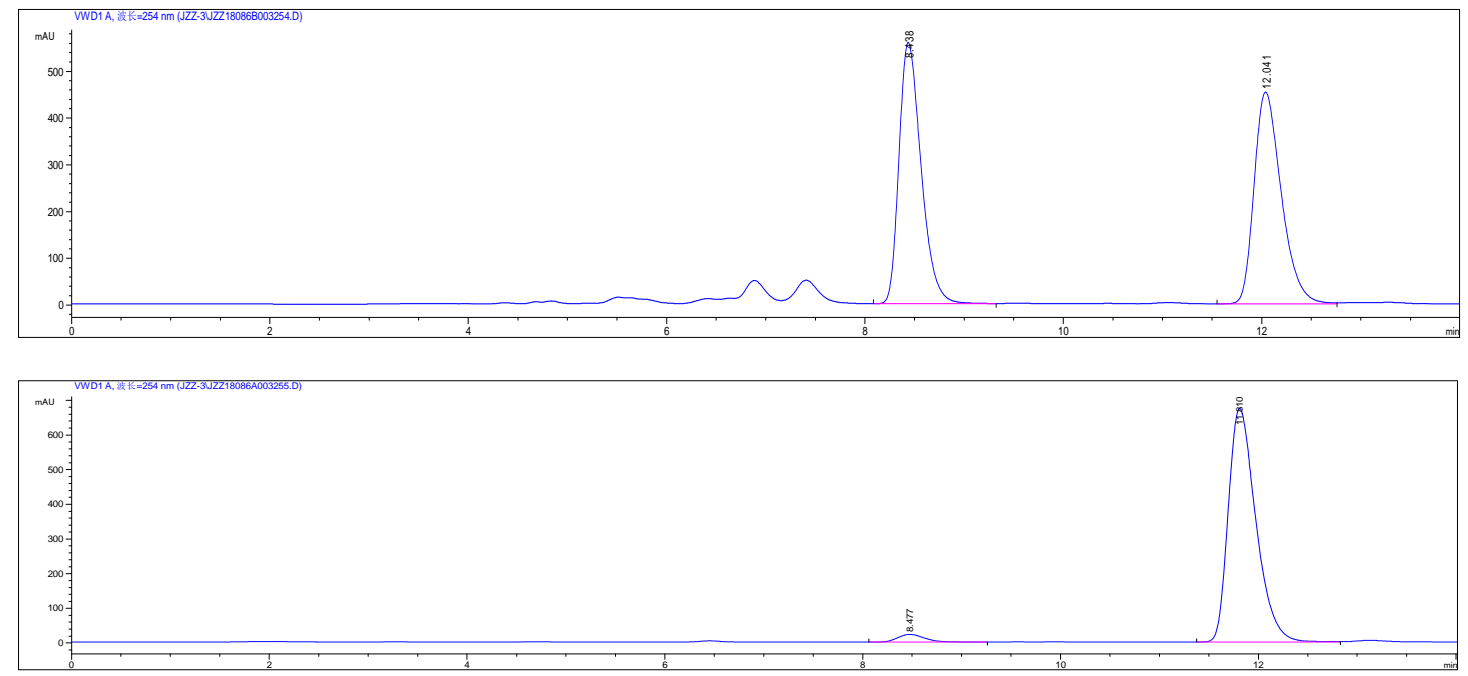

$\underbrace{\mathrm{TH}}_{\mathrm{CF}_{3}}(32.8 \mathrm{mg}, 0.2 \mathrm{mmol}, 2.0$ equiv) and catalyst IV (4.4 mg, $0.01 \mathrm{mmol}$, 3o: $\mathrm{Ar}=4-\mathrm{ClPh} \quad 0.1$ equiv) were dissolved in tert-butylbenzene $(1.0 \mathrm{~mL})$ and stirred at $\mathrm{R}=\mathrm{Ph}$

$-10{ }^{\circ} \mathrm{C}$ for $10 \mathrm{~min}$. Then allyl ketone $10(54.2 \mathrm{mg}, 0.3 \mathrm{mmol}, 3.0$ equiv) was added. The reaction mixture was stirred at $-10{ }^{\circ} \mathrm{C}$ and monitored by TLC. Upon complete consumption of trifluoromethyl ketone 2a, the reaction mixture was directly loaded onto a short silica gel column, followed by gradient elution with PE/EA mixture (20/1-5/1 ratio). Removing the solvent in vacuo, afforded product 3o. White solid, Mp 90.1-91.5 ${ }^{\circ} \mathrm{C}$; $32.6 \mathrm{mg}(0.1 \mathrm{mmol}), 92 \%$ yield; $94 \% e e ;[\alpha]_{\mathrm{D}}^{26}-37.2$ (c 2.60, $\left.\mathrm{CHCl}_{3}\right) ;{ }^{1} \mathrm{H}$ NMR $(300 \mathrm{MHz}$, $\left.\mathrm{CDCl}_{3}\right) \delta 7.67-7.63(\mathrm{~m}, 2 \mathrm{H}), 7.57(\mathrm{~d}, J=6.8 \mathrm{~Hz}, 2 \mathrm{H}), 7.47-7.34(\mathrm{~m}, 5 \mathrm{H}), 6.83(\mathrm{~d}, J=15.5$ $\mathrm{Hz}, 1 \mathrm{H}), 6.75-6.65(\mathrm{~m}, 1 \mathrm{H}), 3.34(\mathrm{~s}, 1 \mathrm{H}), 3.24-3.17(\mathrm{~m}, 1 \mathrm{H}), 3.12-3.04(\mathrm{~m}, 1 \mathrm{H}) ;{ }^{13} \mathrm{C} \mathrm{NMR}$ $\left(75 \mathrm{MHz}, \mathrm{CDCl}_{3}\right) \delta 189.3,141.3,139.5,135.8,135.4,130.2,130.0,128.9,128.8,128.6$, 126.30 (two peaks), 125.2 (q, $J=284.2 \mathrm{~Hz}$ ), 76.7 (q, $J=28.2 \mathrm{~Hz}$ ), 39.0; HRMS (ESI) m/z 355.0720 $\left(\mathrm{M}+\mathrm{H}^{+}\right)$, calc. for $\mathrm{C}_{18} \mathrm{H}_{15} \mathrm{ClF}_{3} \mathrm{O}_{2}$ 355.0713.

The ee was determined by HPLC analysis. CHIRALPAK IB (4.6 mm i.d. x $250 \mathrm{~mm}$ ); Hexane/2-propanol $=90 / 10$; flow rate $1.0 \mathrm{~mL} / \mathrm{min} ; 25{ }^{\circ} \mathrm{C} ; 254 \mathrm{~nm}$; retention time: $10.9 \mathrm{~min}$ (minor) and $17.6 \mathrm{~min}$ (major).

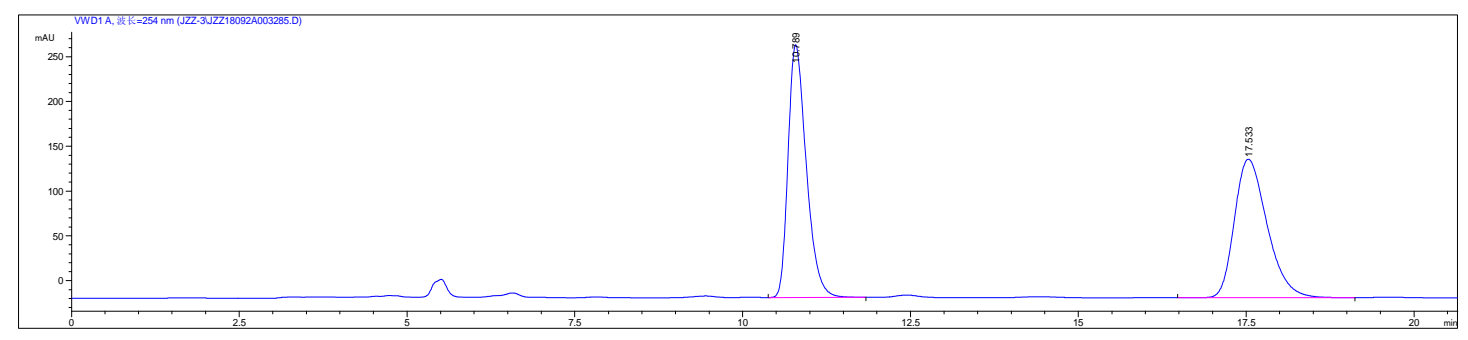




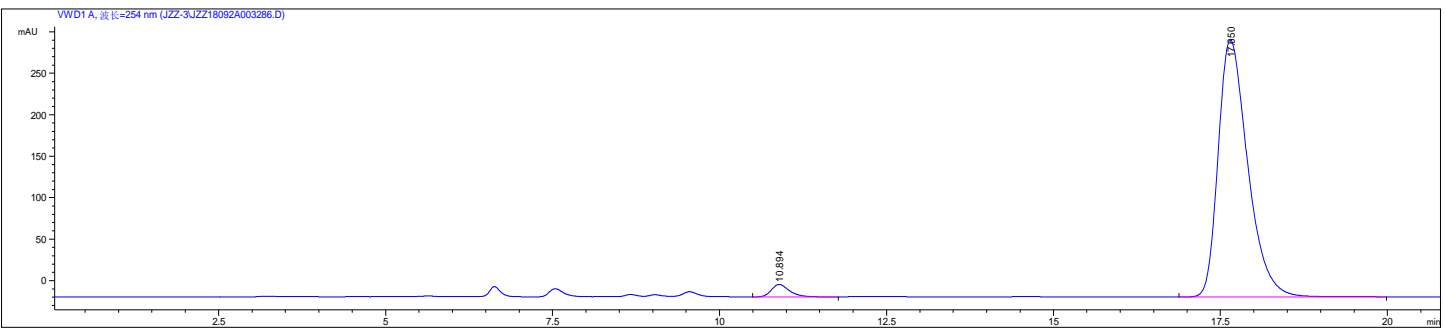

$\mathrm{O} \quad \mathrm{R}^{2} \mathrm{OH} \quad$ Trifluoromethyl ketone 2a (17.4 mg, $0.1 \mathrm{mmol}, 1.0$ equiv), $\mathrm{Na}_{3} \mathrm{PO}_{4}$ $\mathrm{CF}_{3}{ }^{1}(32.8 \mathrm{mg}, 0.2 \mathrm{mmol}, 2.0$ equiv) and catalyst $\mathbf{I V}$ (4.4 mg, $0.01 \mathrm{mmol}$, 3p: Ar = 3-FPh 0.1 equiv) were dissolved in tert-butylbenzene $(1.0 \mathrm{~mL})$ and stirred at $\mathrm{R}=\mathrm{Ph}$ $-10{ }^{\circ} \mathrm{C}$ for $10 \mathrm{~min}$. Then allyl ketone $1 \mathbf{p}(49.3 \mathrm{mg}, 0.3 \mathrm{mmol}, 3.0$ equiv) was added. The reaction mixture was stirred at $-10{ }^{\circ} \mathrm{C}$ and monitored by TLC. Upon complete consumption of trifluoromethyl ketone $\mathbf{2 a}$, the reaction mixture was directly loaded onto a short silica gel column, followed by gradient elution with PE/EA mixture (20/1-5/1 ratio). Removing the solvent in vacuo, afforded product 3p. White solid, $\mathrm{Mp} 87.1-88.9{ }^{\circ} \mathrm{C}$; $31.8 \mathrm{mg}(0.1 \mathrm{mmol}), 94 \%$ yield; $95 \%$ ee; $[\alpha]_{\mathrm{D}}^{26}-27.5\left(c 1.32, \mathrm{CHCl}_{3}\right) ;{ }^{1} \mathrm{H} \mathrm{NMR}(300 \mathrm{MHz}$, $\left.\mathrm{CDCl}_{3}\right) \delta 7.58-7.50(\mathrm{~m}, 3 \mathrm{H}), 7.46-7.35(\mathrm{~m}, 5 \mathrm{H}), 7.23-7.20(\mathrm{~m}, 1 \mathrm{H}), 6.84(\mathrm{~d}, J=15.6 \mathrm{~Hz}$, 1H), 6.77-6.70 (m, 1H), $3.22(\mathrm{~m}, 1 \mathrm{H}), 3.08(\mathrm{~m}, 1 \mathrm{H}), 2.87(\mathrm{~s}, 1 \mathrm{H}) ;{ }^{13} \mathrm{C} \mathrm{NMR}\left(75 \mathrm{MHz}, \mathrm{CDCl}_{3}\right)$ $\delta 189.0,164.4,161.1,141.3,139.4,139.3,135.8,130.4,130.3,130.2,129.0,128.7,126.3$, $125.2(\mathrm{q}, J=283.9 \mathrm{~Hz}), 124.3$ (two peaks), 120.2, 119.9, 115.6, 115.3, 39.1; HRMS (ESI) $\mathrm{m} / \mathrm{z} 339.1010\left(\mathrm{M}+\mathrm{H}^{+}\right)$, calc. for $\mathrm{C}_{18} \mathrm{H}_{15} \mathrm{~F}_{4} \mathrm{O}_{2} 339.1008$.

The ee was determined by HPLC analysis. CHIRALPAK IB (4.6 mm i.d. x $250 \mathrm{~mm}$ ); Hexane/2-propanol $=90 / 10$; flow rate $1.0 \mathrm{~mL} / \mathrm{min} ; 25^{\circ} \mathrm{C} ; 254 \mathrm{~nm}$; retention time: $7.5 \mathrm{~min}$ (minor) and 8.5 min (major).
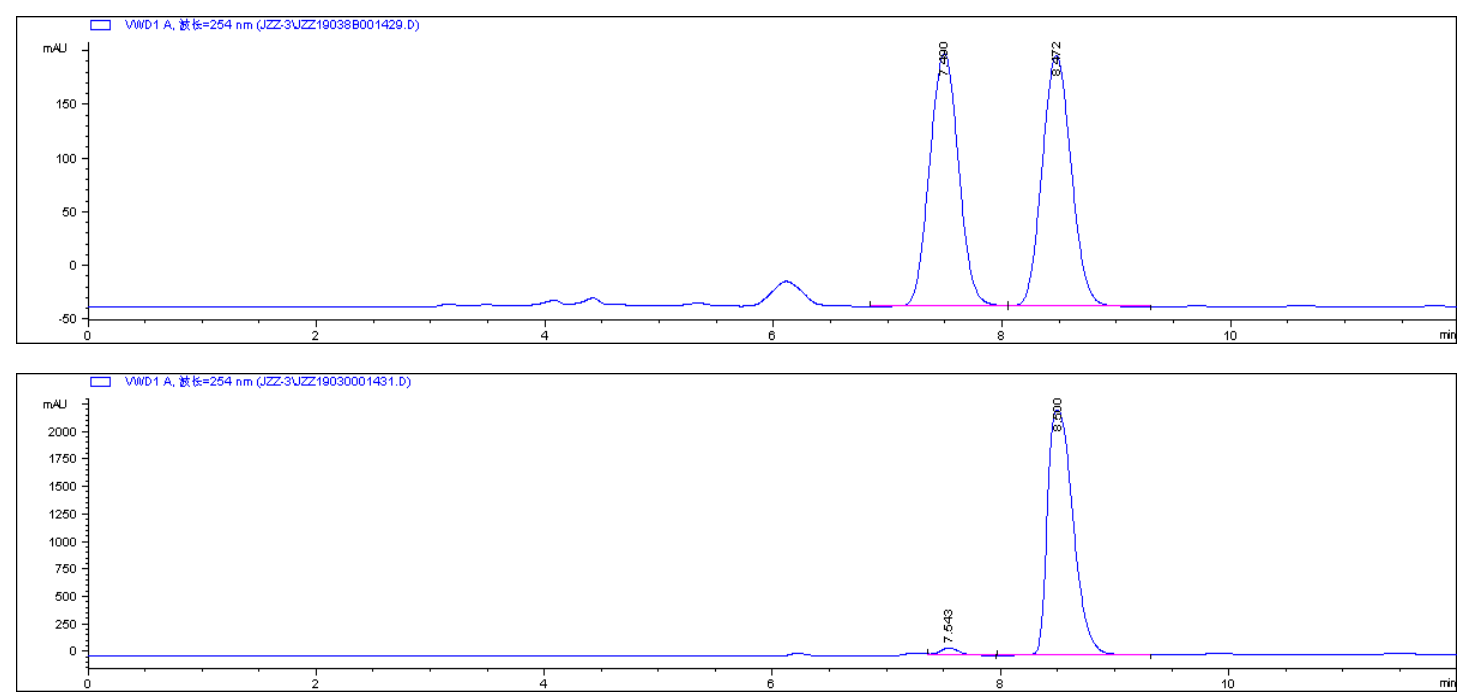
Trifluoromethyl ketone $2 \mathrm{a}\left(17.4 \mathrm{mg}, 0.1 \mathrm{mmol}, 1.0\right.$ equiv), $\mathrm{Na}_{3} \mathrm{PO}_{4}$
$\mathrm{CF}_{3}$

3q: $\mathrm{Ar}=3-\mathrm{CIPh}$

0.1 equiv) were dissolved in tert-butylbenzene $(1.0 \mathrm{~mL})$ and stirred at $\mathrm{R}=\mathrm{Ph}$

$-10{ }^{\circ} \mathrm{C}$ for $10 \mathrm{~min}$. Then allyl ketone $1 \mathrm{q}(54.2 \mathrm{mg}, 0.3 \mathrm{mmol}, 3.0$ equiv) was added. The reaction mixture was stirred at $-10{ }^{\circ} \mathrm{C}$ and monitored by TLC. Upon complete consumption of trifluoromethyl ketone $\mathbf{2 a}$, the reaction mixture was directly loaded onto a short silica gel column, followed by gradient elution with PE/EA mixture (20/1-5/1 ratio). Removing the solvent in vacuo, afforded product 3q. Colorless oil; $33.0 \mathrm{mg}(0.1 \mathrm{mmol})$, 93\% yield; 94\% ee; $[\alpha]_{\mathrm{D}}^{26}-38.2\left(c 1.82, \mathrm{CHCl}_{3}\right) ;{ }^{1} \mathrm{H} \mathrm{NMR}\left(300 \mathrm{MHz}, \mathrm{CDCl}_{3}\right) \delta 7.70(\mathrm{t}, J=$ $1.8 \mathrm{~Hz}, 1 \mathrm{H}), 7.59$ (t, $J=8.2 \mathrm{~Hz}, 3 \mathrm{H}), 7.51-7.31(\mathrm{~m}, 5 \mathrm{H}), 6.83$ (d, $J=15.6 \mathrm{~Hz}, 1 \mathrm{H}), 6.76-6.66$ (m, 1H), 3.25-3.18 (m, 1H), 3.12-3.05 (m, 2H); $\left.{ }^{13} \mathrm{C} \mathrm{NMR} \mathrm{(75} \mathrm{MHz,} \mathrm{CDCl}_{3}\right) \delta 189.0,141.5$, $138.7,135.7,134.8,132.9,130.3,129.8,129.0,128.6,126.6,126.2$ (two peaks), 125.2 (q, $J=$ 284.0 Hz), 76.2, 39.0; HRMS (ESI) m/z 355.0703 $\left(\mathrm{M}+\mathrm{H}^{+}\right)$, calc. for $\mathrm{C}_{18} \mathrm{H}_{15} \mathrm{ClF}_{3} \mathrm{O}_{2} 355.0713$. The ee was determined by HPLC analysis. CHIRALPAK IB (4.6 mm i.d. x $250 \mathrm{~mm}$ ); Hexane/2-propanol $=90 / 10$; flow rate $1.0 \mathrm{~mL} / \mathrm{min} ; 25^{\circ} \mathrm{C}$; $254 \mathrm{~nm}$; retention time: $7.8 \mathrm{~min}$ (minor) and $8.8 \mathrm{~min}$ (major).

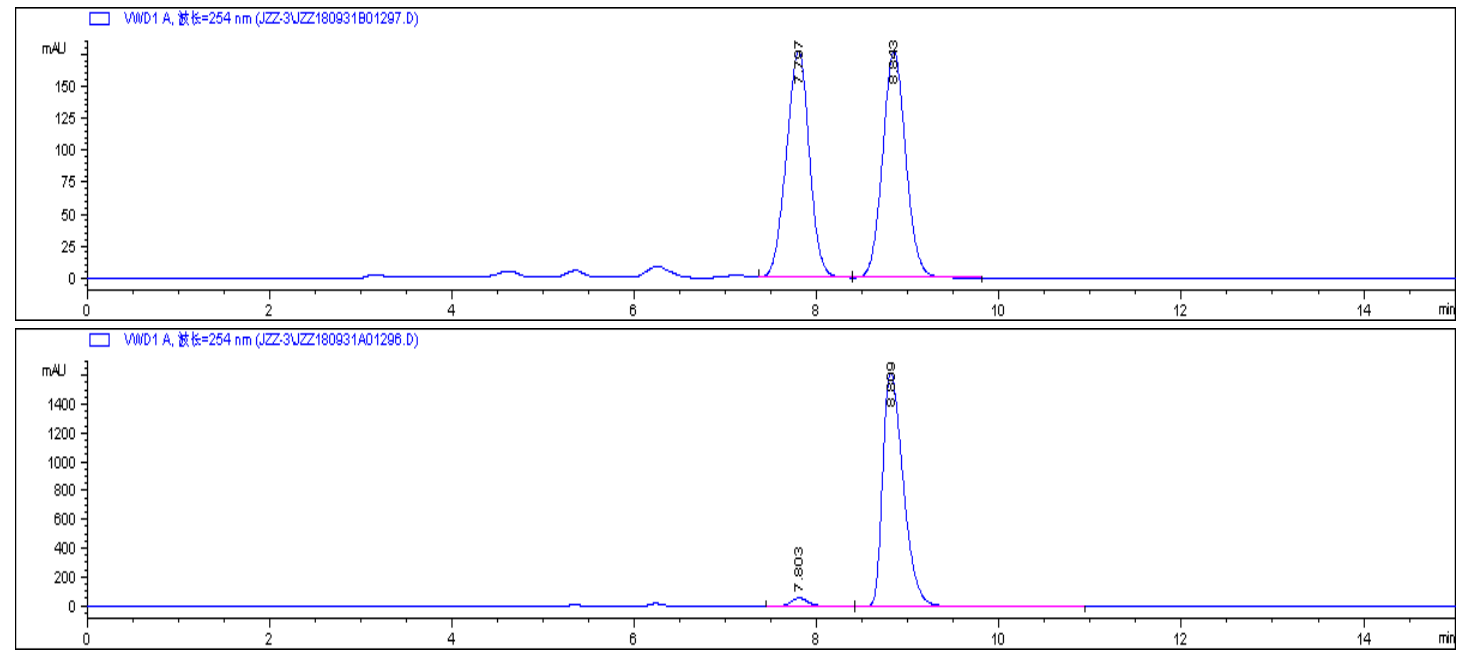

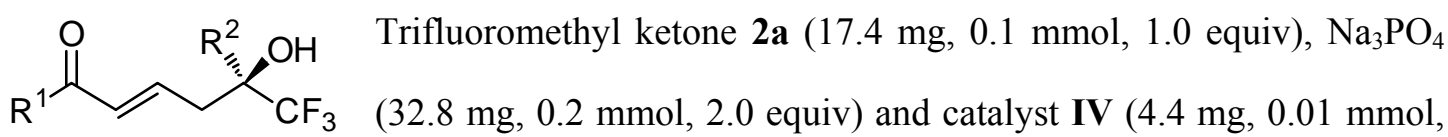
$3 r: A r=2-F P h$ 0.1 equiv) were dissolved in tert-butylbenzene $(1.0 \mathrm{~mL})$ and stirred at $\mathrm{R}=\mathrm{Ph}$ $-10{ }^{\circ} \mathrm{C}$ for $10 \mathrm{~min}$. Then allyl ketone $1 \mathbf{r}(49.3 \mathrm{mg}, 0.3 \mathrm{mmol}, 3.0$ equiv) was added. The reaction mixture was stirred at $-10{ }^{\circ} \mathrm{C}$ and monitored by TLC. Upon complete consumption of trifluoromethyl ketone 2a, the reaction mixture was directly loaded 
onto a short silica gel column, followed by gradient elution with PE/EA mixture (20/1-5/1 ratio). Removing the solvent in vacuo, afforded product 3r. White solid, Mp 74.0-75.9 ${ }^{\circ} \mathrm{C}$; $29.4 \mathrm{mg}(0.1 \mathrm{mmol}), 87 \%$ yield; $83 \%$ ee; $[\alpha]_{\mathrm{D}}^{26}-40.5\left(c \quad 1.86, \mathrm{CHCl}_{3}\right) ;{ }^{1} \mathrm{H} \mathrm{NMR}(300 \mathrm{MHz}$, $\left.\mathrm{CDCl}_{3}\right) \delta 7.64-7.55(\mathrm{~m}, 3 \mathrm{H}), 7.52-7.35(\mathrm{~m}, 4 \mathrm{H}), 7.20-7.05(\mathrm{~m}, 2 \mathrm{H}), 6.83(\mathrm{dd}, J=15.5,3.0$ $\mathrm{Hz}, 1 \mathrm{H}), 6.73-6.64(\mathrm{~m}, 1 \mathrm{H}), 3.23-3.16(\mathrm{~m}, 1 \mathrm{H}), 3.07-3.03(\mathrm{~m}, 1 \mathrm{H}), 2.96(\mathrm{~s}, 1 \mathrm{H}) ;{ }^{13} \mathrm{C} \mathrm{NMR}$ $\left(75 \mathrm{MHz}, \mathrm{CDCl}_{3}\right) \delta 188.4,162.9,159.5,140.6,135.8,134.3,134.2,134.0,133.9,131.0$, 130.9, 128.9, 128.5, 126.3 (two peaks), 125.1 (q, $J=284.2 \mathrm{~Hz}$ ), 124.5, 124.4, 116.6, 116.3, $76.6(\mathrm{q}, J=28.3 \mathrm{~Hz}), 38.9$; HRMS (ESI) $\mathrm{m} / \mathrm{z} 339.1009\left(\mathrm{M}+\mathrm{H}^{+}\right)$, calc. for $\mathrm{C}_{18} \mathrm{H}_{15} \mathrm{~F}_{4} \mathrm{O}_{2}$ 339.1008 .

The ee was determined by HPLC analysis. CHIRALPAK IB (4.6 mm i.d. x $250 \mathrm{~mm}$ ); Hexane $/ 2$-propanol $=95 / 05$; flow rate $1.0 \mathrm{~mL} / \mathrm{min} ; 25{ }^{\circ} \mathrm{C} ; 254 \mathrm{~nm}$; retention time: $11.1 \mathrm{~min}$ (minor) and 12.6 min (major).
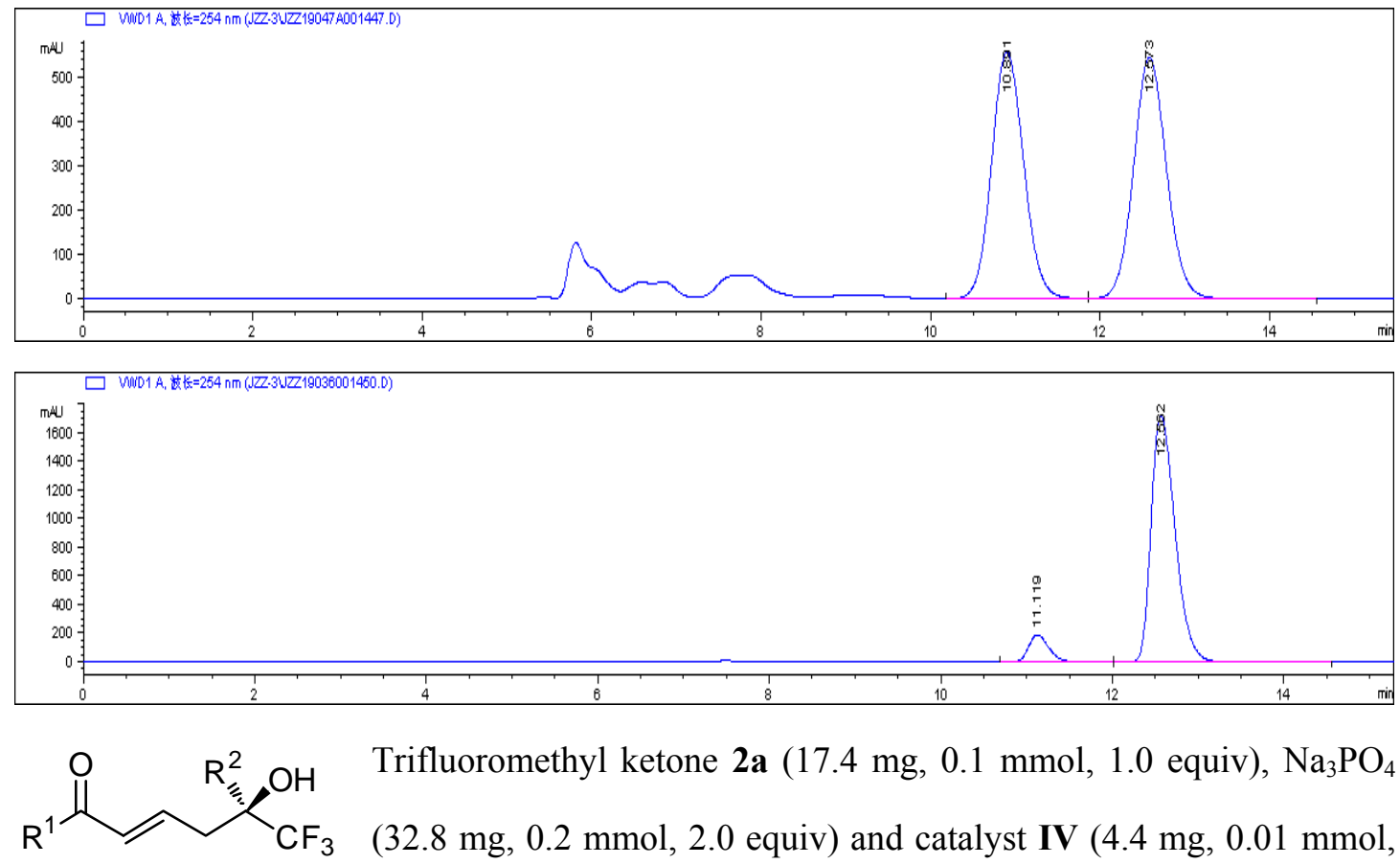

3s: $\mathrm{Ar}=4-\mathrm{MePh}$ 0.1 equiv) were dissolved in tert-butylbenzene $(1.0 \mathrm{~mL})$ and stirred at $\mathrm{R}=\mathrm{Ph}$

$-10{ }^{\circ} \mathrm{C}$ for $10 \mathrm{~min}$. Then allyl ketone $1 \mathrm{~s}(48.1 \mathrm{mg}, 0.3 \mathrm{mmol}, 3.0$ equiv) was added. The reaction mixture was stirred at $-10{ }^{\circ} \mathrm{C}$ and monitored by TLC. Upon complete consumption of trifluoromethyl ketone $\mathbf{2 a}$, the reaction mixture was directly loaded onto a short silica gel column, followed by gradient elution with PE/EA mixture (20/1-5/1 ratio). Removing the solvent in vacuo, afforded product 3s. White solid, Mp 106.2-107. ${ }^{\circ} \mathrm{C}$; 
$27.4 \mathrm{mg}(0.1 \mathrm{mmol}), 82 \%$ yield; $94 \% e e ;[\alpha]_{\mathrm{D}}^{26}-33.2\left(c 1.69, \mathrm{CHCl}_{3}\right) ;{ }^{1} \mathrm{H} \mathrm{NMR}(300 \mathrm{MHz}$, $\left.\mathrm{CDCl}_{3}\right) \delta 7.67(\mathrm{~d}, J=8.2 \mathrm{~Hz}, 2 \mathrm{H}), 7.58(\mathrm{~d}, J=7.2 \mathrm{~Hz}, 2 \mathrm{H}), 7.45-7.38(\mathrm{~m}, 3 \mathrm{H}), 7.20(\mathrm{~d}, J=$ $8.0 \mathrm{~Hz}, 2 \mathrm{H}), 6.74-6.64(\mathrm{~m}, 1 \mathrm{H}), 6.78-6.61(\mathrm{~m}, 1 \mathrm{H}), 3.24-3.04(\mathrm{~m}, 3 \mathrm{H}), 2.39(\mathrm{~s}, 3 \mathrm{H}) ;{ }^{13} \mathrm{C}$ $\operatorname{NMR}\left(75 \mathrm{MHz}, \mathrm{CDCl}_{3}\right) \delta 189.8,143.9,140.0,136.0,134.6,130.8,129.2,128.8$ (two peaks), 126.3 (two peaks), 125.2 (q, $J=284.1 \mathrm{~Hz}$ ), 76.7, 39.0, 21.6; HRMS (ESI) m/z 335.1261 $\left(\mathrm{M}+\mathrm{H}^{+}\right)$, calc. for $\mathrm{C}_{19} \mathrm{H}_{18} \mathrm{~F}_{3} \mathrm{O}_{2} 335.1259$.

The ee was determined by HPLC analysis. CHIRALPAK IB (4.6 mm i.d. x $250 \mathrm{~mm}$ ); Hexane/2-propanol $=90 / 10$; flow rate $1.0 \mathrm{~mL} / \mathrm{min} ; 25{ }^{\circ} \mathrm{C} ; 254 \mathrm{~nm}$; retention time: $12.6 \mathrm{~min}$ (minor) and $16.5 \min$ (major).
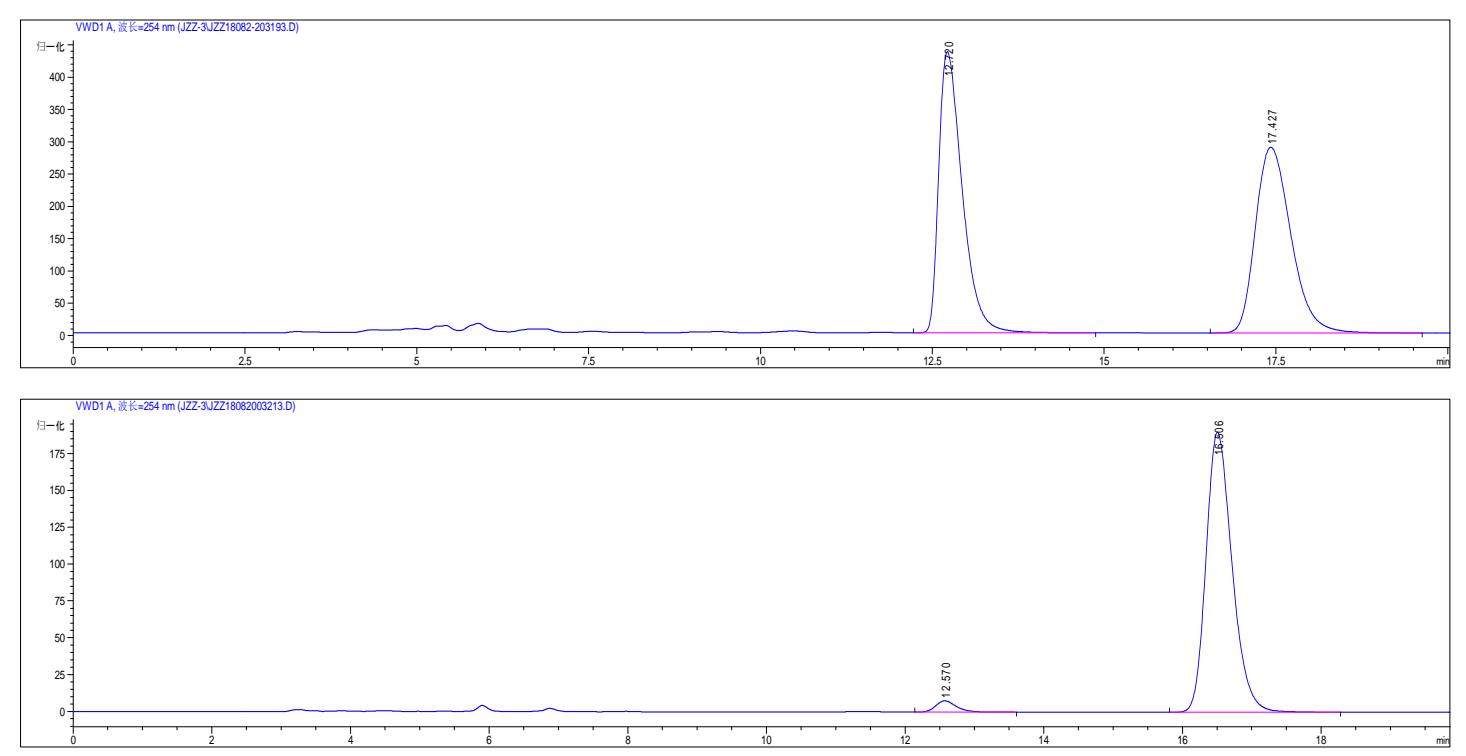

Trifluoromethyl ketone $2 \mathrm{a}\left(17.4 \mathrm{mg}, 0.1 \mathrm{mmol}, 1.0\right.$ equiv), $\mathrm{Na}_{3} \mathrm{PO}_{4}$
$\mathrm{CF}_{3}$ 3t: $\mathrm{Ar}=3-\mathrm{MePh}$ 0.1 equiv) were dissolved in tert-butylbenzene $(1.0 \mathrm{~mL})$ and stirred at $\mathrm{R}=\mathrm{Ph}$ $-10{ }^{\circ} \mathrm{C}$ for $10 \mathrm{~min}$. Then allyl ketone $1 \mathrm{t}(48.1 \mathrm{mg}, 0.3 \mathrm{mmol}, 3.0$ equiv) was added. The reaction mixture was stirred at $-10{ }^{\circ} \mathrm{C}$ and monitored by TLC. Upon complete consumption of trifluoromethyl ketone $\mathbf{2 a}$, the reaction mixture was directly loaded onto a short silica gel column, followed by gradient elution with PE/EA mixture (20/1-5/1 ratio). Removing the solvent in vacuo, afforded product 3t. Colorless oil; $26.7 \mathrm{mg}(0.1 \mathrm{mmol})$, $80 \%$ yield; $93 \% e e ;[\alpha]_{\mathrm{D}}^{26}-40.7\left(\right.$ c 2.46, $\left.\mathrm{CHCl}_{3}\right) ;{ }^{1} \mathrm{H} \mathrm{NMR}\left(300 \mathrm{MHz}, \mathrm{CDCl}_{3}\right) \delta 7.59-7.53(\mathrm{~m}$, 4H), 7.45-7.28 (m, 5H), 6.89 (d, $J=15.5 \mathrm{~Hz}, 1 \mathrm{H}), 6.74-6.64(\mathrm{~m}, 1 \mathrm{H}), 3.25-3.18(\mathrm{~m}, 1 \mathrm{H})$, $3.15(\mathrm{~s}, 1 \mathrm{H}), 3.12-3.04(\mathrm{~m}, 1 \mathrm{H}), 2.36(\mathrm{~s}, 3 \mathrm{H}) ;{ }^{13} \mathrm{C} \mathrm{NMR}\left(75 \mathrm{MHz}, \mathrm{CDCl}_{3}\right) \delta$ 190.6, 140.2, 
138.4, 137.2, 135.9, 133.8, 131.0, 130.9, 129.2, 128.8, 128.6, 128.4, 126.3 (two peaks), 125.8, $125.2(\mathrm{q}, J=284.0 \mathrm{~Hz}), 76.7(\mathrm{q}, J=28.2 \mathrm{~Hz}), 39.0,21.3$; HRMS (ESI) m/z $335.1257\left(\mathrm{M}+\mathrm{H}^{+}\right)$, calc. for $\mathrm{C}_{19} \mathrm{H}_{18} \mathrm{~F}_{3} \mathrm{O}_{2} 335.1259$.

The ee was determined by HPLC analysis. CHIRALPAK IB (4.6 mm i.d. x $250 \mathrm{~mm})$; Hexane/2-propanol $=90 / 10$; flow rate $1.0 \mathrm{~mL} / \mathrm{min} ; 25^{\circ} \mathrm{C} ; 254 \mathrm{~nm}$; retention time: $10.5 \mathrm{~min}$ (minor) and $12.0 \mathrm{~min}$ (major).
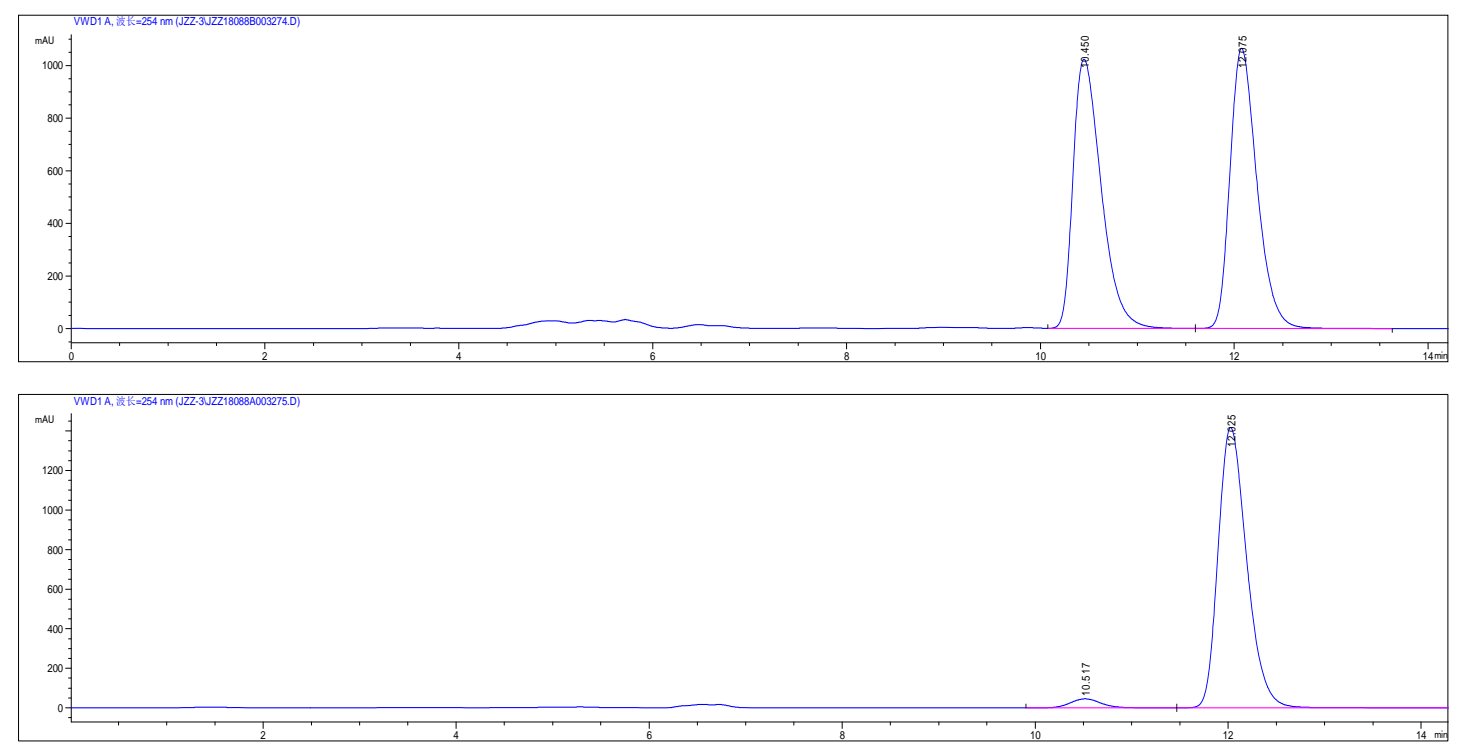

(1) 3u: $\mathrm{Ar}=4-\mathrm{MeOPh}$ 0.1 equiv) were dissolved in tert-butylbenzene $(1.0 \mathrm{~mL})$ and stirred at $\mathrm{R}=\mathrm{Ph}$ $-10{ }^{\circ} \mathrm{C}$ for $10 \mathrm{~min}$. Then allyl ketone $1 \mathbf{u}(52.9 \mathrm{mg}, 0.3 \mathrm{mmol}, 3.0$ equiv) was added. The reaction mixture was stirred at $-10{ }^{\circ} \mathrm{C}$ and monitored by TLC. Upon complete consumption of trifluoromethyl ketone $\mathbf{2 a}$, the reaction mixture was directly loaded onto a short silica gel column, followed by gradient elution with PE/EA mixture (20/1-5/1 ratio). Removing the solvent in vacuo, afforded product $3 \mathbf{u}$. White solid, Mp 107.7-109.2 ${ }^{\circ} \mathrm{C}$; $31.2 \mathrm{mg}(0.1 \mathrm{mmol}), 89 \%$ yield; $94 \% e e ;[\alpha]_{\mathrm{D}}^{26}-39.6$ (c 2.20, $\left.\mathrm{CHCl}_{3}\right) ;{ }^{1} \mathrm{H}$ NMR $(300 \mathrm{MHz}$, $\left.\mathrm{CDCl}_{3}\right) \delta 7.77(\mathrm{~d}, J=8.8 \mathrm{~Hz}, 2 \mathrm{H}), 7.58(\mathrm{~d}, J=7.0 \mathrm{~Hz}, 2 \mathrm{H}), 7.43-7.35(\mathrm{~m}, 3 \mathrm{H}), 6.93-6.86(\mathrm{~m}$, $3 \mathrm{H}), 6.73-6.63(\mathrm{~m}, 1 \mathrm{H}), 3.85(\mathrm{~s}, 3 \mathrm{H}), 3.33(\mathrm{~s}, 1 \mathrm{H}), 3.23-3.2(\mathrm{~m}, 1 \mathrm{H}), 3.11-3.04(\mathrm{~m}, 1 \mathrm{H}) ;{ }^{13} \mathrm{C}$ $\operatorname{NMR}\left(75 \mathrm{MHz}, \mathrm{CDCl}_{3}\right) \delta 188.6,163.6,139.4,136.0,131.00130 .6,130.0,128.8,128.5$, 127.1, 126.4 (two, peaks), 125.2 (q, $J=284.0 \mathrm{~Hz}$ ), 113.8, 76.7 (q, $J=28.1 \mathrm{~Hz}$ ), 55.4, 38.9; HRMS (ESI) m/z $351.1212\left(\mathrm{M}+\mathrm{H}^{+}\right)$, calc. for $\mathrm{C}_{19} \mathrm{H}_{18} \mathrm{~F}_{3} \mathrm{O}_{2} 351.1208$. 
The ee was determined by HPLC analysis. CHIRALPAK IB (4.6 mm i.d. x $250 \mathrm{~mm}$ ); Hexane $/ 2$-propanol $=90 / 10$; flow rate $1.0 \mathrm{~mL} / \mathrm{min} ; 25{ }^{\circ} \mathrm{C} ; 254 \mathrm{~nm}$; retention time: $23.8 \mathrm{~min}$ (minor) and 35.7 min (major).
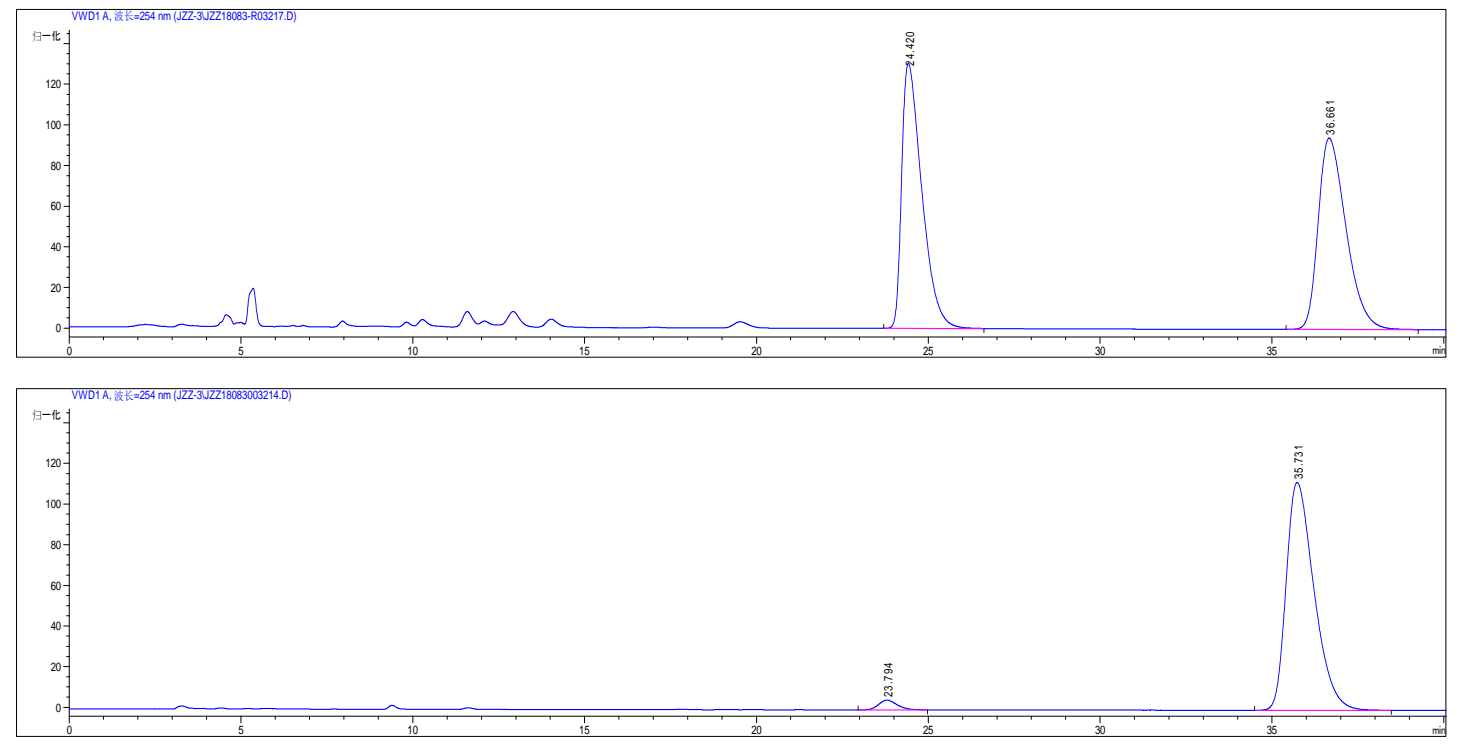

管 3v: $\mathrm{Ar}=3$-MeOPh 0.1 equiv) were dissolved in tert-butylbenzene $(1.0 \mathrm{~mL})$ and stirred at $\mathrm{R}=\mathrm{Ph}$ $-10{ }^{\circ} \mathrm{C}$ for $10 \mathrm{~min}$. Then allyl ketone $1 \mathbf{v}(52.9 \mathrm{mg}, 0.3 \mathrm{mmol}, 3.0$ equiv) was added. The reaction mixture was stirred at $-10{ }^{\circ} \mathrm{C}$ and monitored by TLC. Upon complete consumption of trifluoromethyl ketone $\mathbf{2 a}$, the reaction mixture was directly loaded onto a short silica gel column, followed by gradient elution with PE/EA mixture (20/1-5/1 ratio). Removing the solvent in vacuo, afforded product 3v. Colorless oil; $31.9 \mathrm{mg}(0.1 \mathrm{mmol})$, 91\% yield; 94\% ee; $\left.[\alpha]_{\mathrm{D}}^{26}-36.3(c) 2.76, \mathrm{CHCl}_{3}\right) ;{ }^{1} \mathrm{H} \mathrm{NMR}\left(300 \mathrm{MHz}, \mathrm{CDCl}_{3}\right) \delta 7.57(\mathrm{~d}, J=$ $7.1 \mathrm{~Hz}, 2 \mathrm{H}), 7.45-7.38(\mathrm{~m}, 3 \mathrm{H}), 7.33-7.31(\mathrm{~m}, 3 \mathrm{H}), 7.11-7.06(\mathrm{~m}, 1 \mathrm{H}), 6.89$ (d, $J=15.5 \mathrm{~Hz}$ 1H), 6.77-6.67 (m, 1H), $3.81(\mathrm{~s}, 3 \mathrm{H}), 3.25-3.18(\mathrm{~m}, 1 \mathrm{H}), 3.12-3.04(\mathrm{~m}, 2 \mathrm{H}),{ }^{13} \mathrm{C}$ NMR $(75$ $\left.\mathrm{MHz}, \mathrm{CDCl}_{3}\right) \delta 190.0,159.8,140.5,138.6,135.9,130.8,129.5,128.9,128.6,126.3,125.2(\mathrm{q}$, $J=284.2 \mathrm{~Hz}), 121.3,119.7,112.8,76.7(\mathrm{q}, J=28.2 \mathrm{~Hz}), 55.4,39.0 ;$ HRMS (ESI) $\mathrm{m} / \mathrm{z}$ $351.1211\left(\mathrm{M}+\mathrm{H}^{+}\right)$, calc. for $\mathrm{C}_{19} \mathrm{H}_{18} \mathrm{~F}_{3} \mathrm{O}_{3} 351.1208$.

The ee was determined by HPLC analysis. CHIRALPAK IB (4.6 mm i.d. x $250 \mathrm{~mm}$ ); Hexane/2-propanol $=90 / 10$; flow rate $1.0 \mathrm{~mL} / \mathrm{min} ; 25^{\circ} \mathrm{C} ; 254 \mathrm{~nm}$; retention time: $18.5 \mathrm{~min}$ (minor) and $21.1 \mathrm{~min}$ (major). 

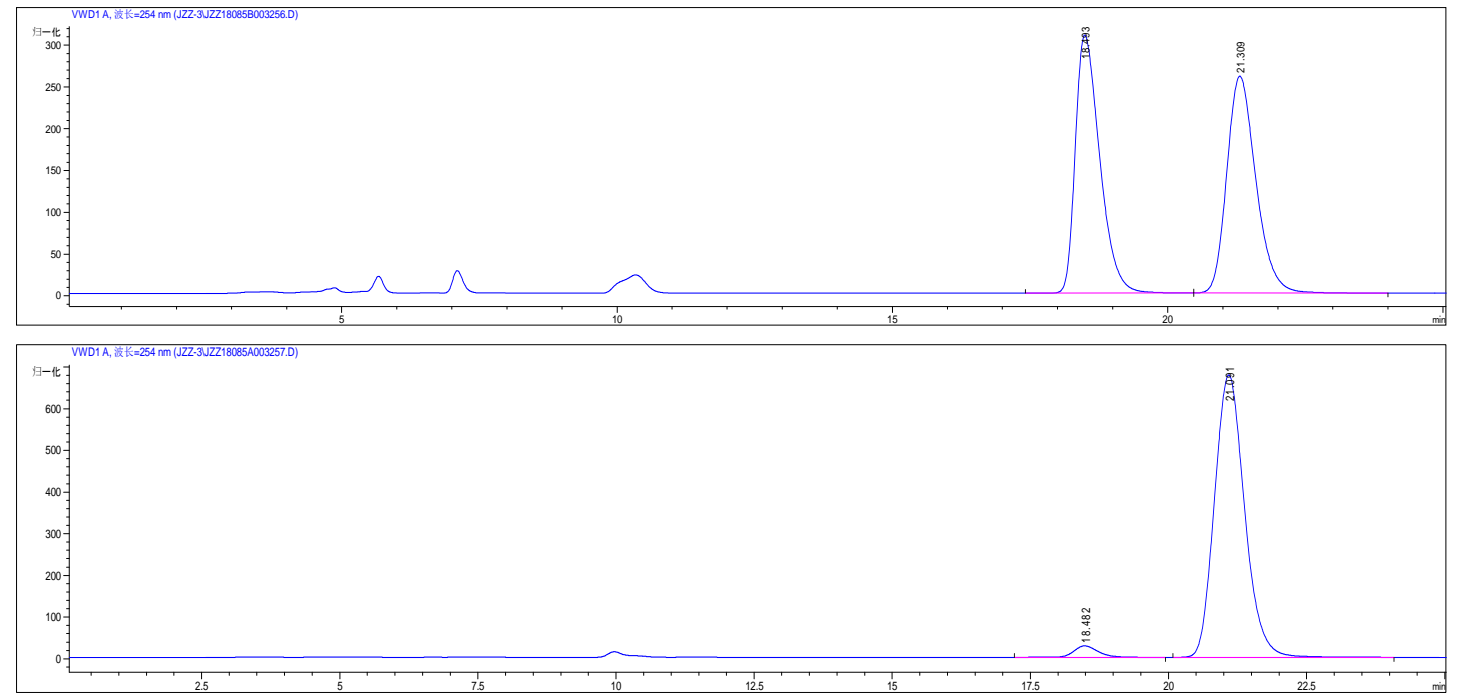

Trifluoromethyl ketone 2a (17.4 mg, $0.1 \mathrm{mmol}, 1.0$ equiv), $\mathrm{Na}_{3} \mathrm{PO}_{4}$

$3 \mathrm{w}: \mathrm{Ar}=2-\mathrm{MeOPh}$

0.1 equiv) were dissolved in tert-butylbenzene $(1.0 \mathrm{~mL})$ and stirred at $\mathrm{R}=\mathrm{Ph}$

$-10{ }^{\circ} \mathrm{C}$ for $10 \mathrm{~min}$. Then allyl ketone $1 \mathbf{w}(52.9 \mathrm{mg}, 0.3 \mathrm{mmol}, 3.0$ equiv) was added. The reaction mixture was stirred at $-10{ }^{\circ} \mathrm{C}$ and monitored by TLC. Upon complete consumption of trifluoromethyl ketone $\mathbf{2 a}$, the reaction mixture was directly loaded onto a short silica gel column, followed by gradient elution with PE/EA mixture (20/1-5/1 ratio). Removing the solvent in vacuo, afforded product 3w. Colorless oil; $21.0 \mathrm{mg}(0.1$ mmol), 60\% yield; 70\% ee; $[\alpha]_{\mathrm{D}}^{26}-38.3\left(c 1.17, \mathrm{CHCl}_{3}\right) ;{ }^{1} \mathrm{H} \mathrm{NMR}\left(300 \mathrm{MHz}, \mathrm{CDCl}_{3}\right) \delta 7.57$ $(\mathrm{d}, J=7.0 \mathrm{~Hz}, 2 \mathrm{H}), 7.48-7.34(\mathrm{~m}, 5 \mathrm{H}), 7.00-6.91(\mathrm{~m}, 2 \mathrm{H}), 6.83(\mathrm{~d}, J=15.6 \mathrm{~Hz}, 1 \mathrm{H})$, 6.59-6.48 (m, 1H), $3.83(\mathrm{~s}, 3 \mathrm{H}), 3.18-3.11(\mathrm{~m}, 1 \mathrm{H}), 3.08-3.01(\mathrm{~m}, 1 \mathrm{H}), 2.93(\mathrm{~s}, 1 \mathrm{H}) ;{ }^{13} \mathrm{C}$ $\operatorname{NMR}\left(75 \mathrm{MHz}, \mathrm{CDCl}_{3}\right) \delta 192.0,158.1,137.8,136.2,136.1,133.3,130.5,128.8,128.5,128.2$, 126.3, 125.1 (q, $J=283.9 \mathrm{~Hz}), 120.8,111.5,76.4(\mathrm{q}, J=28.1 \mathrm{~Hz}), 55.7,38.8$; HRMS (ESI) $\mathrm{m} / \mathrm{z} 351.1204\left(\mathrm{M}+\mathrm{H}^{+}\right)$, calc. for $\mathrm{C}_{19} \mathrm{H}_{18} \mathrm{~F}_{3} \mathrm{O}_{3} 351.1208$.

The ee was determined by HPLC analysis. CHIRALPAK IB (4.6 mm i.d. x $250 \mathrm{~mm})$; Hexane/2-propanol $=90 / 10$; flow rate $1.0 \mathrm{~mL} / \mathrm{min} ; 25^{\circ} \mathrm{C} ; 254 \mathrm{~nm}$; retention time: $10.6 \mathrm{~min}$ (minor) and $11.7 \mathrm{~min}$ (major).

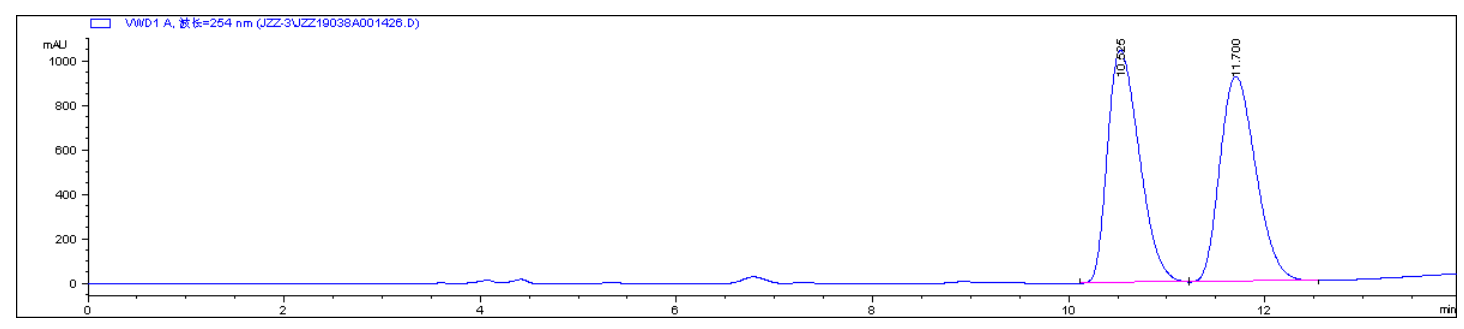




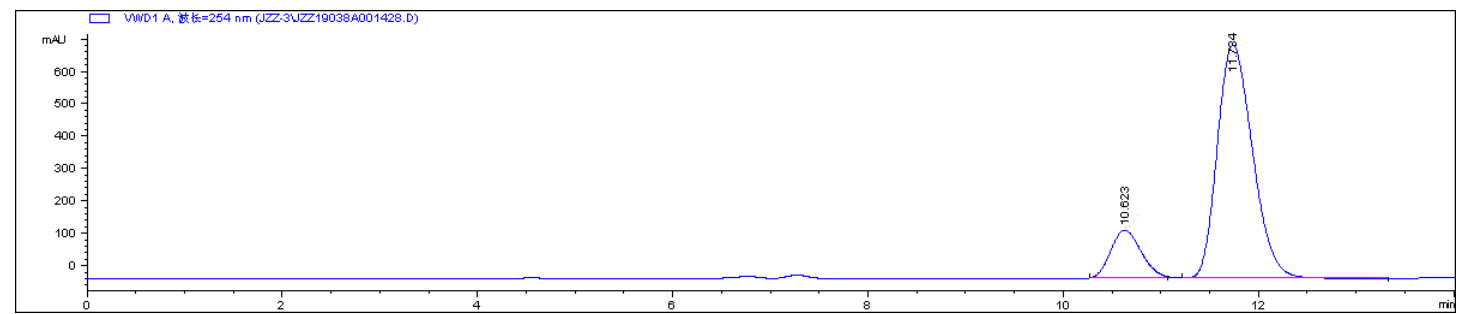

$\mathrm{O} \quad \mathrm{R}_{2}^{2} \mathrm{OH} \quad$ Trifluoromethyl ketone $2 \mathrm{a}\left(17.4 \mathrm{mg}, 0.1 \mathrm{mmol}, 1.0\right.$ equiv), $\mathrm{Na}_{3} \mathrm{PO}_{4}$ $\mathrm{CF}_{3} \quad(32.8 \mathrm{mg}, 0.2 \mathrm{mmol}, 2.0$ equiv) and catalyst IV (4.4 mg, $0.01 \mathrm{mmol}$,

3x: Ar = 2-naphtyl

0.1 equiv) were dissolved in tert-butylbenzene $(1.0 \mathrm{~mL})$ and stirred at $\mathrm{R}=\mathrm{Ph}$ $-10{ }^{\circ} \mathrm{C}$ for $10 \mathrm{~min}$. Then allyl ketone $1 \mathrm{x}(58.9 \mathrm{mg}, 0.3 \mathrm{mmol}, 3.0$ equiv) was added. The reaction mixture was stirred at $-10{ }^{\circ} \mathrm{C}$ and monitored by TLC. Upon complete consumption of trifluoromethyl ketone 2a, the reaction mixture was directly loaded onto a short silica gel column, followed by gradient elution with PE/EA mixture (20/1-5/1 ratio). Removing the solvent in vacuo, afforded product 3x. White solid, Mp 91.4-93.2 ${ }^{\circ} \mathrm{C}$; $35.9 \mathrm{mg}(0.1 \mathrm{mmol}), 97 \%$ yield; $94 \%$ ee; $[\alpha]_{\mathrm{D}}^{26}-28.0\left(\right.$ c 3.13, $\left.\mathrm{CHCl}_{3}\right) ;{ }^{1} \mathrm{H}$ NMR (300 MHz, $\left.\mathrm{CDCl}_{3}\right) \delta 8.20(\mathrm{~s}, 1 \mathrm{H}), 7.87-7.80(\mathrm{~m}, 4 \mathrm{H}), 7.63-7.37(\mathrm{~m}, 7 \mathrm{H}), 7.04(\mathrm{~d}, J=15.5 \mathrm{~Hz}, 1 \mathrm{H})$, 6.83-6.73 (m, 1H), $3.43(\mathrm{~d}, J=3.3 \mathrm{~Hz}, 1 \mathrm{H}), 3.30-3.22(\mathrm{~m}, 1 \mathrm{H}), 3.17-3.10(\mathrm{~m}, 1 \mathrm{H}) ;{ }^{13} \mathrm{C} \mathrm{NMR}$ $\left(75 \mathrm{MHz}, \mathrm{CDCl}_{3}\right) \delta 190.3,140.5,136.0,135.5,134.4,132.3,130.8,130.4,129.5,128.9$, 128.6, 128.5 (two peaks), 126.7, 126.4 (two peaks), 125.2 (q, $J=283.9 \mathrm{~Hz}$ ), 76.7 (q, $J=28.1$ Hz), 38.9; HRMS (ESI) m/z 371.1258 $\left(\mathrm{M}+\mathrm{H}^{+}\right)$, calc. for $\mathrm{C}_{22} \mathrm{H}_{18} \mathrm{~F}_{3} \mathrm{O}_{2} 371.1259$.

The ee was determined by HPLC analysis. CHIRALPAK IB (4.6 mm i.d. x $250 \mathrm{~mm}$ ); Hexane/2-propanol $=90 / 10$; flow rate $1.0 \mathrm{~mL} / \mathrm{min} ; 25^{\circ} \mathrm{C} ; 254 \mathrm{~nm}$; retention time: $22.1 \mathrm{~min}$ (minor) and $33.2 \mathrm{~min}$ (major).
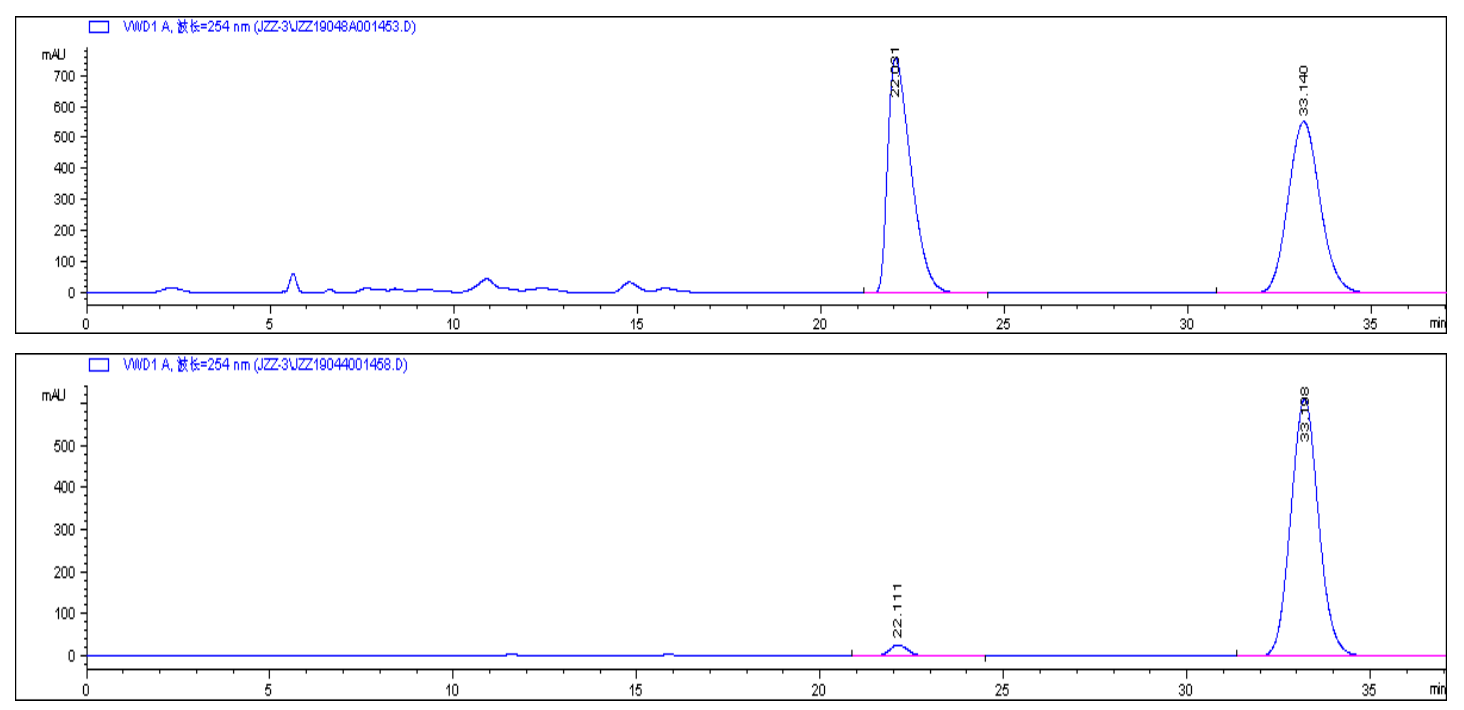


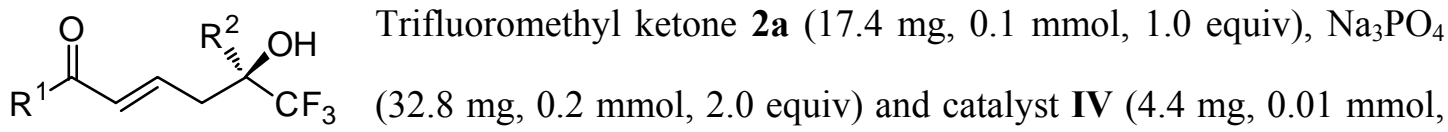

3y: Ar = 2-thienyl

0.1 equiv) were dissolved in tert-butylbenzene $(1.0 \mathrm{~mL})$ and stirred at $\mathrm{R}=\mathrm{Ph}$

$-10{ }^{\circ} \mathrm{C}$ for $10 \mathrm{~min}$. Then allyl ketone $1 \mathbf{y}(45.7 \mathrm{mg}, 0.3 \mathrm{mmol}, 3.0$ equiv) was added. The reaction mixture was stirred at $-10{ }^{\circ} \mathrm{C}$ and monitored by TLC. Upon complete consumption of trifluoromethyl ketone 2a, the reaction mixture was directly loaded onto a short silica gel column, followed by gradient elution with PE/EA mixture (20/1-5/1 ratio). Removing the solvent in vacuo, afforded product $\mathbf{3 y}$. White solid, Mp 126.3-128.0 ${ }^{\circ} \mathrm{C}$; $32.0 \mathrm{mg}(0.1 \mathrm{mmol}), 98 \%$ yield; $99 \% e e ;[\alpha]_{\mathrm{D}}^{26}-44.6\left(c\right.$ 2.46, $\left.\mathrm{CHCl}_{3}\right) ;{ }^{1} \mathrm{H}$ NMR $(300 \mathrm{MHz}$, $\left.\mathrm{CDCl}_{3}\right) \delta$ 7.64-7.56 (m, 4H), 7.44-7.34 (m, 3H), 7.11-7.08 (m, 1H), $6.84(\mathrm{~d}, J=15.3 \mathrm{~Hz}$, 1H), 6.80-6.73 (m, 1H), 3.26-3.18 (m, 2H), 3.11-3.05 (m, 1H); ${ }^{13} \mathrm{C} \mathrm{NMR} \mathrm{(75} \mathrm{MHz,} \mathrm{CDCl}_{3}$ ) $\delta 181.7,144.4,139.9,135.9,134.4,132.5,130.1,128.9,128.5,128.2,126.3,125.2(\mathrm{q}, J=$ $284.1 \mathrm{~Hz}), 76.6(\mathrm{q}, J=28.3 \mathrm{~Hz}), 38.8$; HRMS (ESI) $\mathrm{m} / \mathrm{z} 327.0666\left(\mathrm{M}+\mathrm{H}^{+}\right)$, calc. for $\mathrm{C}_{16} \mathrm{H}_{14} \mathrm{~F}_{3} \mathrm{O}_{2} \mathrm{~S} 327.0667$.

The ee was determined by HPLC analysis. CHIRALPAK IB (4.6 mm i.d. x $250 \mathrm{~mm}$ ); Hexane/2-propanol $=90 / 10$; flow rate $1.0 \mathrm{~mL} / \mathrm{min} ; 25{ }^{\circ} \mathrm{C} ; 254 \mathrm{~nm}$; retention time: $30.6 \mathrm{~min}$ (minor) and 31.9 min (major).
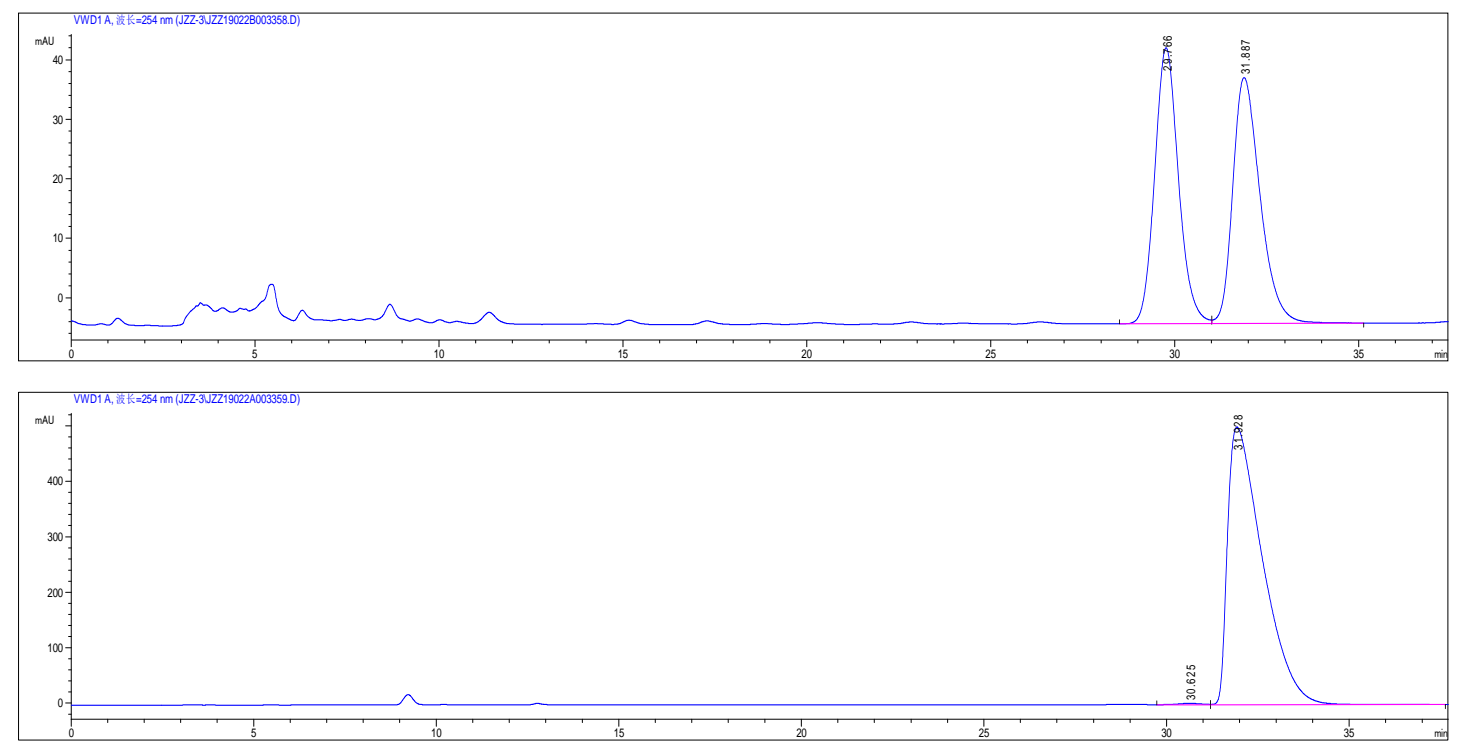<smiles>CC(=O)C(O)(C/C=C/C(=O)c1ccccc1)c1ccccc1</smiles>

$6 a$
Ketone 5a (16.4 mg, $0.1 \mathrm{mmol}, 1.0$ equiv), $\mathrm{K}_{2} \mathrm{HPO}_{4}(34.8 \mathrm{mg}$, $0.2 \mathrm{mmol}, 2.0$ equiv) and catalyst $\mathbf{I V}(4.4 \mathrm{mg}, 0.01 \mathrm{mmol}, 0.1$ equiv) were dissolved in tert-butylbenzene $(0.8 \mathrm{~mL})$ and stirred 
at $-20{ }^{\circ} \mathrm{C}$ for $10 \mathrm{~min}$. Then allyl ketone $1 \mathrm{a}$ (43.9 mg, $0.3 \mathrm{mmol}, 3.0$ equiv) was added. The reaction mixture was stirred at $-20{ }^{\circ} \mathrm{C}$ and monitored by TLC. Upon complete consumption of ketone $\mathbf{5}$, the reaction mixture was directly loaded onto a short silica gel column, followed by gradient elution with PE/EA mixture (20/1-5/1 ratio). Removing the solvent in vacuo, afforded product 6a. Colorless oil; $24.2 \mathrm{mg}(0.1 \mathrm{mmol}), 78 \%$ yield; $89 \% e e ;[\alpha]_{\mathrm{D}}^{26}+5.8(c 1.27$, $\left.\mathrm{CHCl}_{3}\right) ;{ }^{1} \mathrm{H}$ NMR $\left(300 \mathrm{MHz}, \mathrm{CDCl}_{3}\right) \delta 7.85-7.82(\mathrm{~m}, 2 \mathrm{H}), 7.63-7.52(\mathrm{~m}, 3 \mathrm{H}), 7.46-7.30(\mathrm{~m}$, 5H), 6.92-6.90 (m, 2H), $3.82(\mathrm{~s}, 4 \mathrm{H}), 3.22-3.15(\mathrm{~m}, 1 \mathrm{H}), 3.06-3.00(\mathrm{~m}, 1 \mathrm{H}) ;{ }^{13} \mathrm{C}$ NMR $(75$ $\left.\mathrm{MHz}, \mathrm{CDCl}_{3}\right) \delta 190.7,174.5,142.6,140.8,137.6,132.7,129.9,128.6,128.5$ (two peaks), 128.2, 125.3, 78.0, 53.5, 43.0; HRMS (ESI) $\mathrm{m} / \mathrm{z} 333.1092\left(\mathrm{M}+\mathrm{H}^{+}\right)$, calc. for $\mathrm{C}_{19} \mathrm{H}_{18} \mathrm{O}_{4} \mathrm{Na}$ 333.1103.

The ee was determined by HPLC analysis. CHIRALPAK IC (4.6 mm i.d. x $250 \mathrm{~mm}$ ); Hexane/2-propanol $=80 / 20$; flow rate $1.0 \mathrm{~mL} / \mathrm{min} ; 25{ }^{\circ} \mathrm{C} ; 254 \mathrm{~nm}$; retention time: $20.7 \mathrm{~min}$ (minor) and $23.6 \mathrm{~min}$ (major).
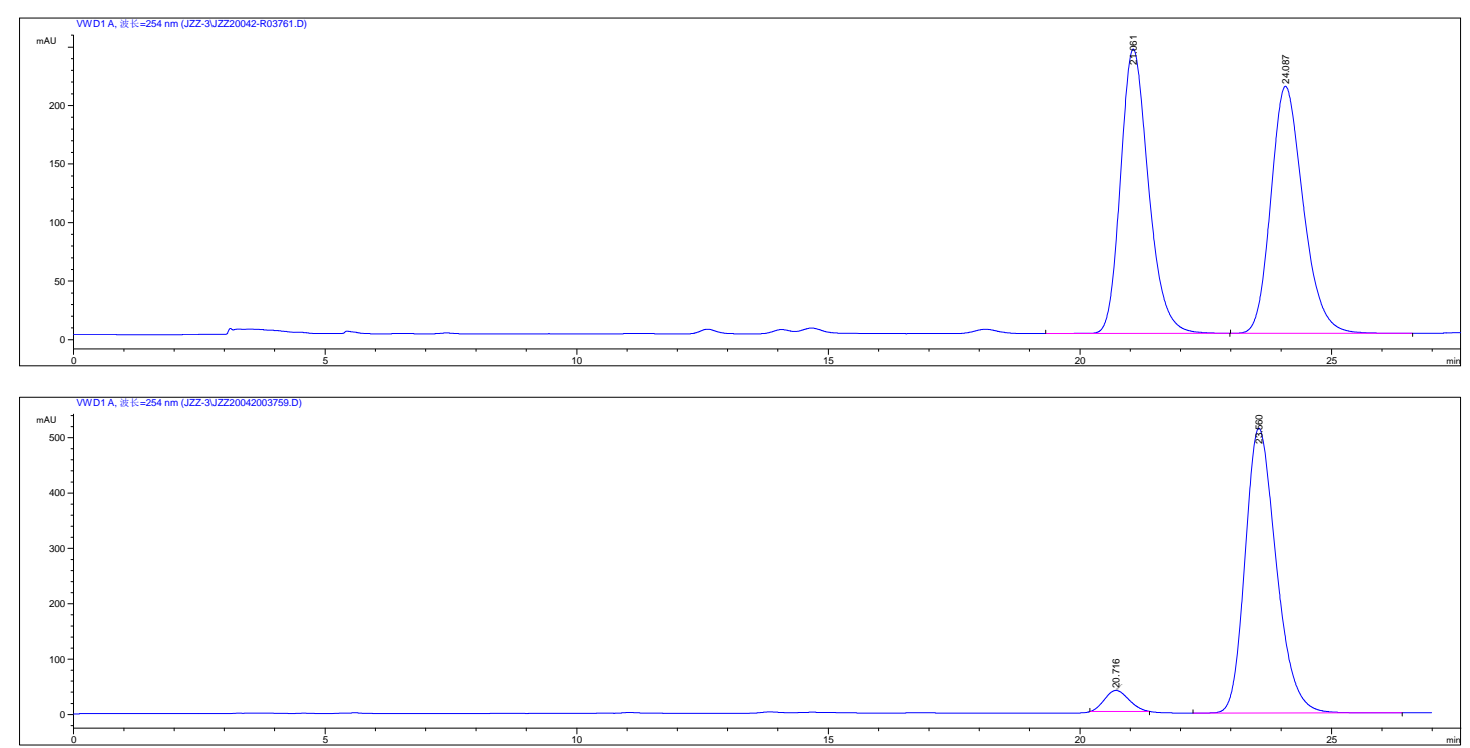<smiles>CC(=O)[C@](O)(C/C=C/C(=O)c1cccs1)c1ccccc1</smiles>

$6 b$

Ketone 5a (16.4 mg, $0.1 \mathrm{mmol}, 1.0$ equiv), $\mathrm{K}_{2} \mathrm{HPO}_{4}(34.8 \mathrm{mg}$, $0.2 \mathrm{mmol}, 2.0$ equiv) and catalyst IV (4.4 mg, $0.01 \mathrm{mmol}, 0.1$ equiv) were dissolved in tert-butylbenzene $(0.8 \mathrm{~mL})$ and stirred at $-20{ }^{\circ} \mathrm{C}$ for $10 \mathrm{~min}$. Then allyl ketone $1 \mathbf{y}(45.7 \mathrm{mg}$, TLC. Upon complete consumption of ketone 5a, the reaction mixture was directly loaded onto a short silica gel column, followed by gradient elution with PE/EA mixture (20/1-5/1 
ratio). Removing the solvent in vacuo, afforded product $\mathbf{6 b}$. Colorless oil; $23.7 \mathrm{mg}(0.1 \mathrm{mmol})$, $75 \%$ yield; 94\% ee; $[\alpha]_{\mathrm{D}}^{26}+7.2\left(c 0.78, \mathrm{CHCl}_{3}\right) ;{ }^{1} \mathrm{H} \mathrm{NMR}\left(300 \mathrm{MHz}, \mathrm{CDCl}_{3}\right) \delta 7.69(\mathrm{dd}, J=$ 3.8, 1.0 Hz, 1H), 7.65-7.58 (m, 3H), 7.41-7.29 (m, 3H), 7.14-7.12 (m, 1H), 7.04-6.94 (m, $1 \mathrm{H}), 6.85(\mathrm{~d}, J=15.4 \mathrm{~Hz}, 1 \mathrm{H}), 3.82(\mathrm{~s}, 4 \mathrm{H}), 3.23-3.16(\mathrm{~m}, 1 \mathrm{H}), 3.05-2.97(\mathrm{~m}, 1 \mathrm{H}) ;{ }^{13} \mathrm{C} \mathrm{NMR}$ $\left(75 \mathrm{MHz}, \mathrm{CDCl}_{3}\right) \delta 182.0,174.4,144.8,141.8,140.8,134.0,132.2,129.2,128.5,128.2$ (two peaks), 125.3, 77.9, 53.6, 42.8; HRMS (ESI) $\mathrm{m} / \mathrm{z} 317.0847\left(\mathrm{M}+\mathrm{H}^{+}\right)$, calc. for $\mathrm{C}_{17} \mathrm{H}_{17} \mathrm{O}_{4} \mathrm{~S}$ 317.0848 .

The ee was determined by HPLC analysis. CHIRALPAK IC (4.6 mm i.d. x $250 \mathrm{~mm}$ ); Hexane/2-propanol $=80 / 20$; flow rate $1.0 \mathrm{~mL} / \mathrm{min} ; 25{ }^{\circ} \mathrm{C} ; 254 \mathrm{~nm}$; retention time: $28.9 \mathrm{~min}$ (minor) and 31.9 min (major).
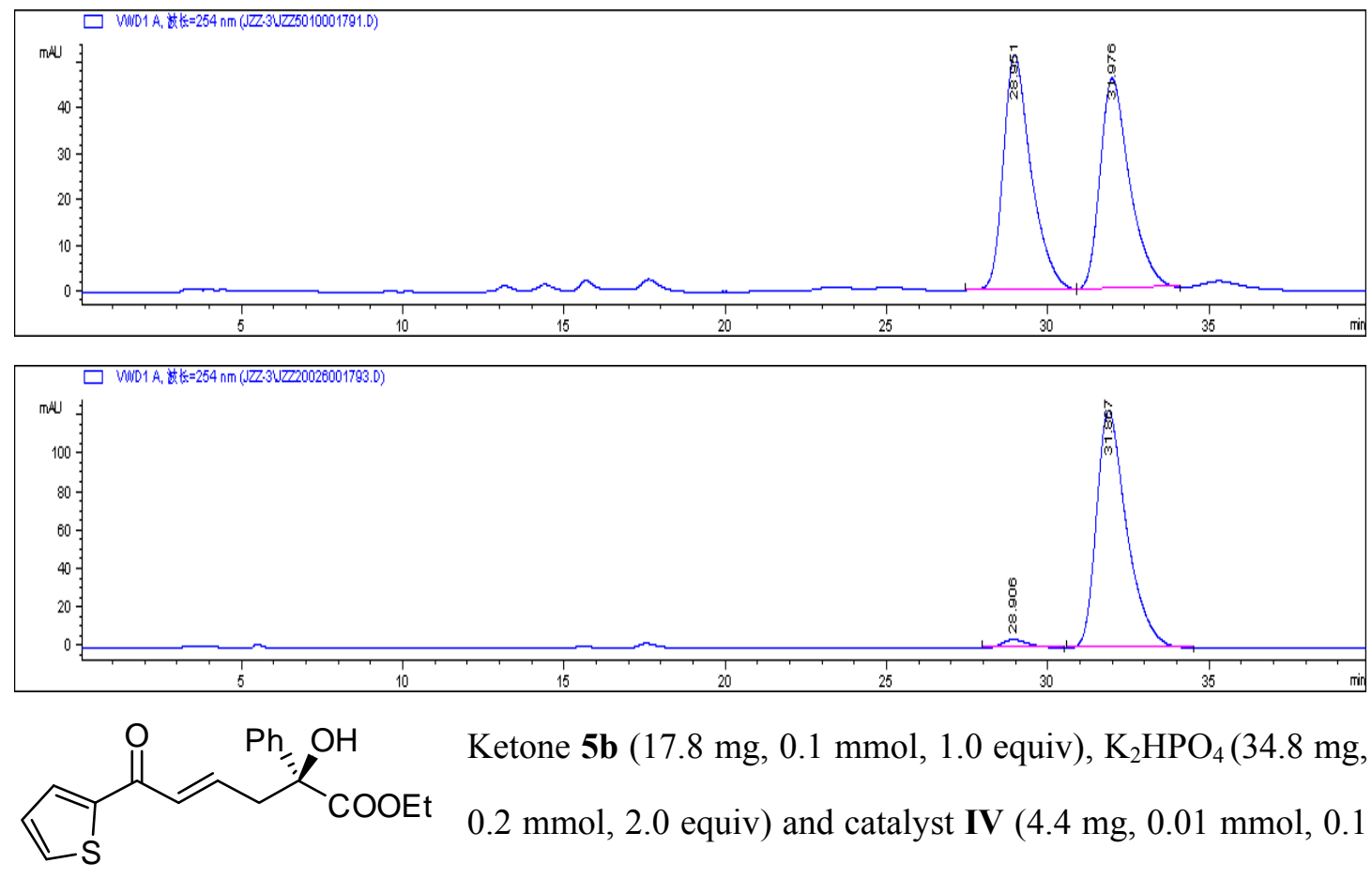

Ketone 5b (17.8 mg, 0.1 mmol, 1.0 equiv), $\mathrm{K}_{2} \mathrm{HPO}_{4}(34.8 \mathrm{mg}$, $0.2 \mathrm{mmol}, 2.0$ equiv) and catalyst IV (4.4 mg, $0.01 \mathrm{mmol}, 0.1$ 6c equiv) were dissolved in tert-butylbenzene $(0.8 \mathrm{~mL})$ and stirred at $-20{ }^{\circ} \mathrm{C}$ for $10 \mathrm{~min}$. Then allyl ketone $1 \mathbf{y}(45.7 \mathrm{mg}$, $0.3 \mathrm{mmol}, 3.0$ equiv) was added. The reaction mixture was stirred at $-20{ }^{\circ} \mathrm{C}$ and monitored by TLC. Upon complete consumption of ketone $\mathbf{5 b}$, the reaction mixture was directly loaded onto a short silica gel column, followed by gradient elution with PE/EA mixture (20/1-5/1 ratio). Removing the solvent in vacuo, afforded product 6c. Colorless oil; $25.1 \mathrm{mg}$ (0.1 mmol), $76 \%$ yield; 94\% ee; $[\alpha]_{\mathrm{D}}^{26}+4.102\left(c 1.51, \mathrm{CHCl}_{3}\right) ;{ }^{1} \mathrm{H} \mathrm{NMR}\left(300 \mathrm{MHz}, \mathrm{CDCl}_{3}\right) \delta 7.69(\mathrm{~d}, J=$ $3.8 \mathrm{~Hz}, 1 \mathrm{H}), 7.65-7.60(\mathrm{~m}, 3 \mathrm{H}), 7.41-7.29(\mathrm{~m}, 3 \mathrm{H}), 7.15-7.12(\mathrm{~m}, 1 \mathrm{H}), 7.06-6.96(\mathrm{~m}, 1 \mathrm{H})$, 
$6.86(\mathrm{~d}, J=15.4 \mathrm{~Hz}, 1 \mathrm{H}), 4.36-4.18(\mathrm{~m}, 2 \mathrm{H}), 3.85(\mathrm{~s}, 1 \mathrm{H}), 3.22-3.16(\mathrm{~m}, 1 \mathrm{H}), 3.04-2.96(\mathrm{~m}$, 1H), $1.64(\mathrm{~s}, 1 \mathrm{H}), 1.29$ (t, $J=7.1 \mathrm{~Hz}, 3 \mathrm{H}) ;{ }^{13} \mathrm{C} \mathrm{NMR}\left(75 \mathrm{MHz}, \mathrm{CDCl}_{3}\right) \delta 182.0,174.0,144.8$, $141.9,140.9,133.9,132.1,129.2,128.4,128.1$ (two peaks), 125.3, 77.7, 62.9, 42.8, 14.1; HRMS (ESI) m/z 353.0823 (M+Na ${ }^{+}$, calc. for $\mathrm{C}_{18} \mathrm{H}_{18} \mathrm{O}_{4} \mathrm{SNa} 353.0824$.

The ee was determined by HPLC analysis. CHIRALPAK IC (4.6 mm i.d. x $250 \mathrm{~mm})$; Hexane/2-propanol $=80 / 20$; flow rate $1.0 \mathrm{~mL} / \mathrm{min} ; 25{ }^{\circ} \mathrm{C} ; 254 \mathrm{~nm}$; retention time: $29.4 \mathrm{~min}$ (minor) and 34.1min (major).
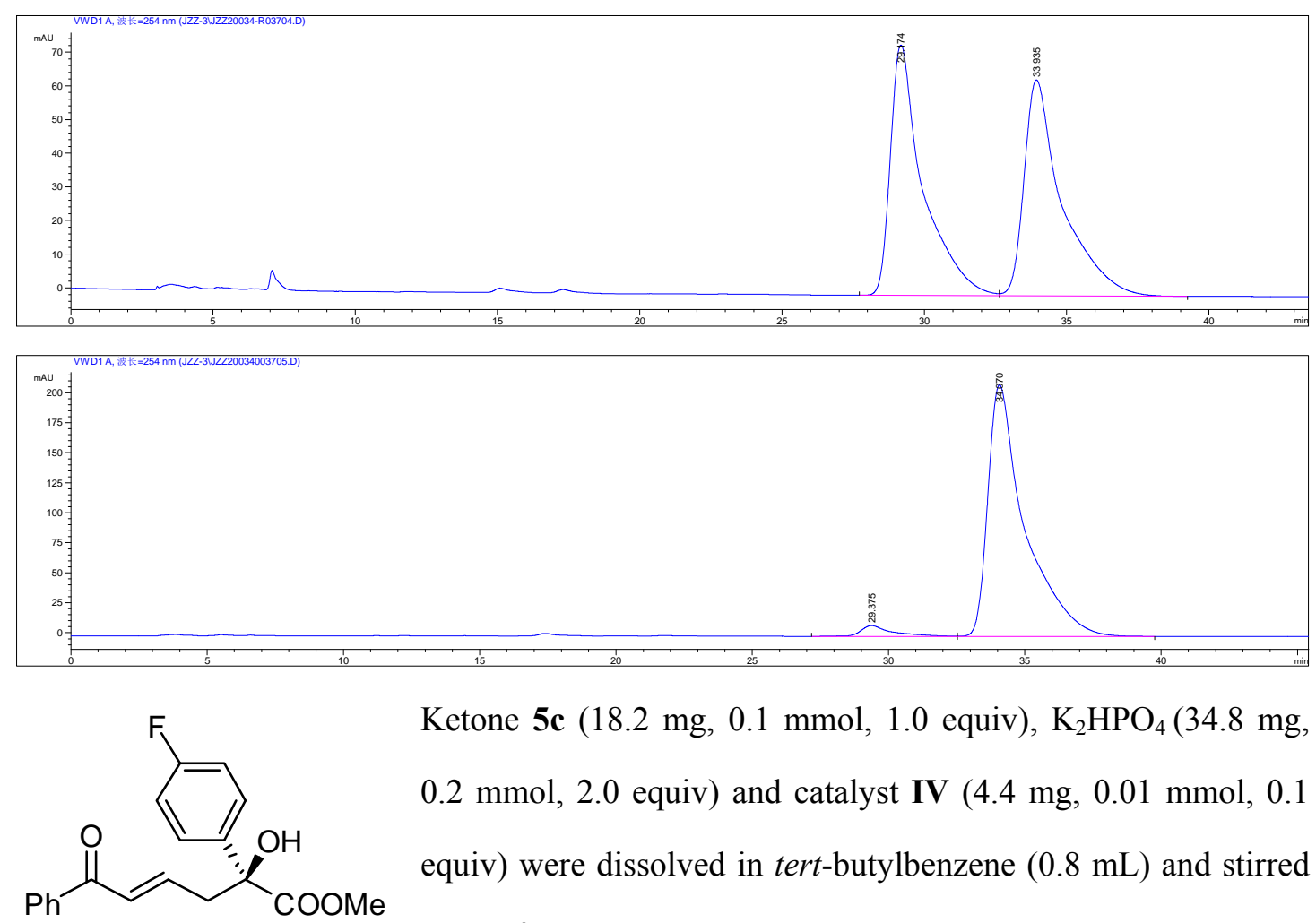

Ketone 5c (18.2 mg, $0.1 \mathrm{mmol}, 1.0$ equiv), $\mathrm{K}_{2} \mathrm{HPO}_{4}(34.8 \mathrm{mg}$, $0.2 \mathrm{mmol}, 2.0$ equiv) and catalyst $\mathbf{I V}(4.4 \mathrm{mg}, 0.01 \mathrm{mmol}, 0.1$ equiv) were dissolved in tert-butylbenzene $(0.8 \mathrm{~mL})$ and stirred at $-20{ }^{\circ} \mathrm{C}$ for $10 \mathrm{~min}$. Then allyl ketone $1 \mathrm{a}(43.9 \mathrm{mg}, 0.3 \mathrm{mmol}$,

6d 3.0 equiv) was added. The reaction mixture was stirred at -20 ${ }^{\circ} \mathrm{C}$ and monitored by TLC. Upon complete consumption of ketone $\mathbf{5 c}$, the reaction mixture was directly loaded onto a short silica gel column, followed by gradient elution with PE/EA mixture (20/1-5/1 ratio). Removing the solvent in vacuo, afforded product $6 \mathbf{d}$. Colorless oil; $24.6 \mathrm{mg}(0.1 \mathrm{mmol}), 75 \%$ yield; $90 \% e e ;[\alpha]_{\mathrm{D}}^{26}+5.5\left(c 1.66, \mathrm{CHCl}_{3}\right) ;{ }^{1} \mathrm{H} \mathrm{NMR}(300 \mathrm{MHz}$, $\left.\mathrm{CDCl}_{3}\right) \delta 7.85-7.83(\mathrm{~m}, 2 \mathrm{H}), 7.61-7.53(\mathrm{~m}, 3 \mathrm{H}), 7.45(\mathrm{t}, J=7.4 \mathrm{~Hz}, 2 \mathrm{H}), 7.09-7.03(\mathrm{~m}, 2 \mathrm{H})$, 6.95-6.82 (m, 2H), $3.84(\mathrm{~s}, 1 \mathrm{H}), 3.82(\mathrm{~s}, 3 \mathrm{H}), 3.19-3.13(\mathrm{~m}, 1 \mathrm{H}), 3.02-2.96(\mathrm{~m}, 1 \mathrm{H}) ;{ }^{13} \mathrm{C}$ $\operatorname{NMR}\left(75 \mathrm{MHz}, \mathrm{CDCl}_{3}\right) \delta 190.6,174.4,164.2,160.9,142.1,137.5,136.5,132.8,130.0,128.6$, 
128.5, 127.3, 127.2, 115.5, 115.2, 77.6, 53.6, 43.1; HRMS (ESI) m/z 351.1011 (M+H'), calc. for $\mathrm{C}_{19} \mathrm{H}_{17} \mathrm{FO}_{4} \mathrm{Na} 351.1009$.

The ee was determined by HPLC analysis. CHIRALPAK IE (4.6 mm i.d. x $250 \mathrm{~mm}$ ); Hexane/2-propanol $=80 / 20$; flow rate $1.0 \mathrm{~mL} / \mathrm{min} ; 25{ }^{\circ} \mathrm{C} ; 254 \mathrm{~nm}$; retention time: $16.8 \mathrm{~min}$ (minor) and 20.4 min (major).
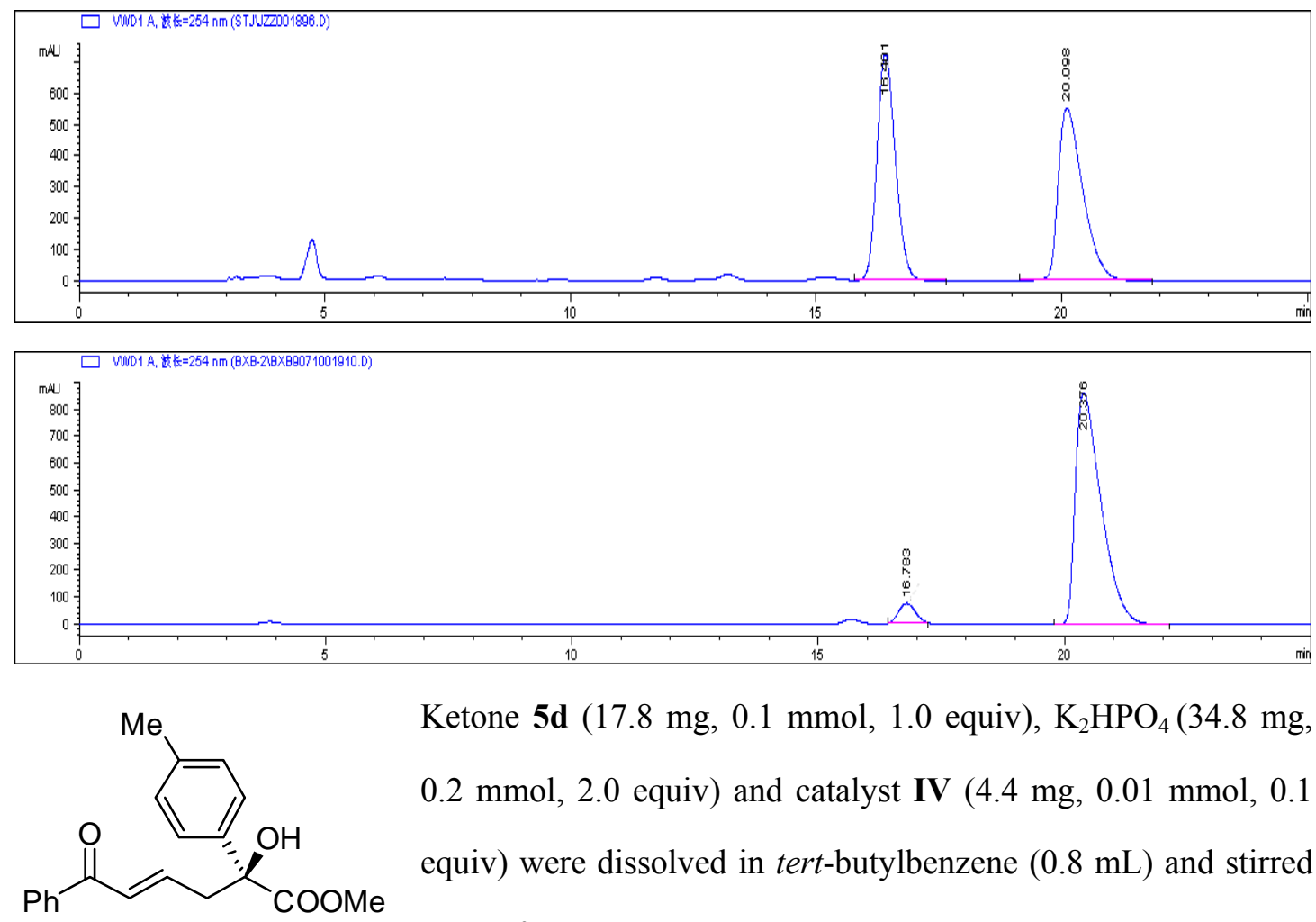

Ketone 5d (17.8 mg, 0.1 mmol, 1.0 equiv), $\mathrm{K}_{2} \mathrm{HPO}_{4}(34.8 \mathrm{mg}$, $0.2 \mathrm{mmol}, 2.0$ equiv) and catalyst $\mathbf{I V}(4.4 \mathrm{mg}, 0.01 \mathrm{mmol}, 0.1$ equiv) were dissolved in tert-butylbenzene $(0.8 \mathrm{~mL})$ and stirred at $-20{ }^{\circ} \mathrm{C}$ for $10 \mathrm{~min}$. Then allyl ketone $1 \mathrm{a}(43.9 \mathrm{mg}, 0.3 \mathrm{mmol}$,

6e $\quad 3.0$ equiv) was added. The reaction mixture was stirred at -20 ${ }^{\circ} \mathrm{C}$ and monitored by TLC. Upon complete consumption of ketone $\mathbf{5 c}$, the reaction mixture was directly loaded onto a short silica gel column, followed by gradient elution with PE/EA mixture (20/1-5/1 ratio). Removing the solvent in vacuo, afforded product 6e. Colorless oil; $20.5 \mathrm{mg}(0.1 \mathrm{mmol}), 60 \%$ yield; $90 \% e e ;[\alpha]_{\mathrm{D}}^{26}-12.9\left(c \mathrm{c} .79, \mathrm{CHCl}_{3}\right) ;{ }^{1} \mathrm{H} \mathrm{NMR}(300 \mathrm{MHz}$, $\left.\mathrm{CDCl}_{3}\right) \delta 7.86-7.83(\mathrm{~m}, 2 \mathrm{H}), 7.57-7.42(\mathrm{~m}, 5 \mathrm{H}), 7.19(\mathrm{~d}, J=8.0 \mathrm{~Hz}, 2 \mathrm{H}), 6.91-6.89(\mathrm{~m}, 2 \mathrm{H})$, $3.81(\mathrm{~s}, 3 \mathrm{H}), 3.77(\mathrm{~s}, 1 \mathrm{H}), 3.20-3.14(\mathrm{~m}, 1 \mathrm{H}), 3.04-2.98(\mathrm{~m}, 1 \mathrm{H}), 2.35(\mathrm{~s}, 3 \mathrm{H}),{ }^{13} \mathrm{C}$ NMR $(75$ $\left.\mathrm{MHz}, \mathrm{CDCl}_{3}\right) \delta 190.7,174.7,142.7,138.0,137.9,137.6,132.7,129.8,129.2,128.6,128.5$, 125.2, 77.9, 53.5, 42.9, 21.0; HRMS (ESI) $\mathrm{m} / \mathrm{z} 347.1263\left(\mathrm{M}+\mathrm{H}^{+}\right)$, calc. for $\mathrm{C}_{20} \mathrm{H}_{20} \mathrm{O}_{4} \mathrm{Na}$ 347.1259 .

The ee was determined by HPLC analysis. CHIRALPAK IB (4.6 mm i.d. x $250 \mathrm{~mm}$ ); 
Hexane/2-propanol $=90 / 10$; flow rate $1.0 \mathrm{~mL} / \mathrm{min} ; 25^{\circ} \mathrm{C} ; 254 \mathrm{~nm}$; retention time: $10.6 \mathrm{~min}$ (minor) and $11.6 \mathrm{~min}$ (major).

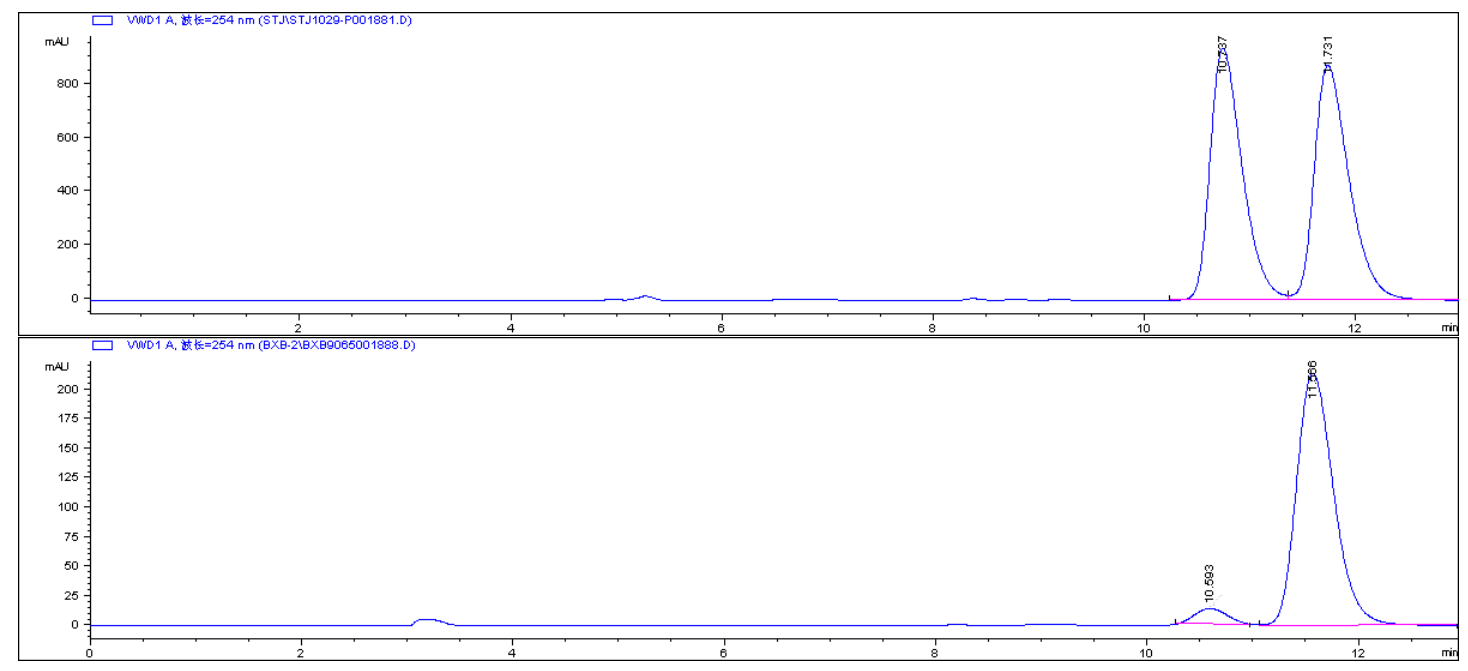<smiles>CC(=O)[C@](O)(C/C=C/C(=O)c1ccccc1)c1cccc(C)c1</smiles>

Ketone 5e (17.8 mg, $0.1 \mathrm{mmol}, 1.0$ equiv), $\mathrm{K}_{2} \mathrm{HPO}_{4}(34.8 \mathrm{mg}$, $0.2 \mathrm{mmol}, 2.0$ equiv) and catalyst $\mathbf{I V}(4.4 \mathrm{mg}, 0.01 \mathrm{mmol}, 0.1$ equiv) were dissolved in tert-butylbenzene $(0.8 \mathrm{~mL})$ and stirred at $-20{ }^{\circ} \mathrm{C}$ for $10 \mathrm{~min}$. Then allyl ketone $1 \mathrm{a}(43.9 \mathrm{mg}, 0.3 \mathrm{mmol}$, 6f 3.0 equiv) was added. The reaction mixture was stirred at -20 ${ }^{\circ} \mathrm{C}$ and monitored by TLC. Upon complete consumption of ketone $\mathbf{5 c}$, the reaction mixture was directly loaded onto a short silica gel column, followed by gradient elution with PE/EA mixture (20/1-5/1 ratio). Removing the solvent in vacuo, afforded product 6f. Colorless oil; $23.0 \mathrm{mg}(0.1 \mathrm{mmol}), 71 \%$ yield; $91 \%$ ee; $[\alpha]_{\mathrm{D}}^{26}-6.2\left(c 1.04, \mathrm{CHCl}_{3}\right) ;{ }^{1} \mathrm{H}$ NMR $(300 \mathrm{MHz}$, $\left.\mathrm{CDCl}_{3}\right) \delta 7.86-7.83(\mathrm{~m}, 2 \mathrm{H}), 7.55(\mathrm{t}, J=7.3 \mathrm{~Hz}, 1 \mathrm{H}), 7.47-7.37(\mathrm{~m}, 4 \mathrm{H}), 7.27(\mathrm{t}, J=7.6 \mathrm{~Hz}$, 1H), $7.14(\mathrm{~d}, J=7.4 \mathrm{~Hz}, 1 \mathrm{H}), 6.92-9.90(\mathrm{~m}, 2 \mathrm{H}), 3.81(\mathrm{~s}, 3 \mathrm{H}), 3.78(\mathrm{~s}, 1 \mathrm{H}), 3.22-3.15(\mathrm{~m}$, 1H), 3.05-2.99 (m, 1H), 2.38 (s, 3H); ${ }^{13} \mathrm{C}$ NMR (75 MHz, $\left.\mathrm{CDCl}_{3}\right) \delta$ 190.7, 174.6, 142.7, $140.7,138.2,137.6,132.7,129.8,128.9,128.6,128.5,128.4,125.9,122.4,78.0,53.5,42.9$, 21.6; HRMS (ESI) m/z $347.1265\left(\mathrm{M}+\mathrm{H}^{+}\right)$, calc. for $\mathrm{C}_{20} \mathrm{H}_{20} \mathrm{O}_{4} \mathrm{Na} 347.1259$.

The ee was determined by HPLC analysis. CHIRALPAK IC (4.6 mm i.d. x $250 \mathrm{~mm}$ ); Hexane/2-propanol $=80 / 20$; flow rate $1.0 \mathrm{~mL} / \mathrm{min} ; 25^{\circ} \mathrm{C} ; 254 \mathrm{~nm}$; retention time: $19.8 \mathrm{~min}$ (minor) and $23.4 \mathrm{~min}$ (major). 


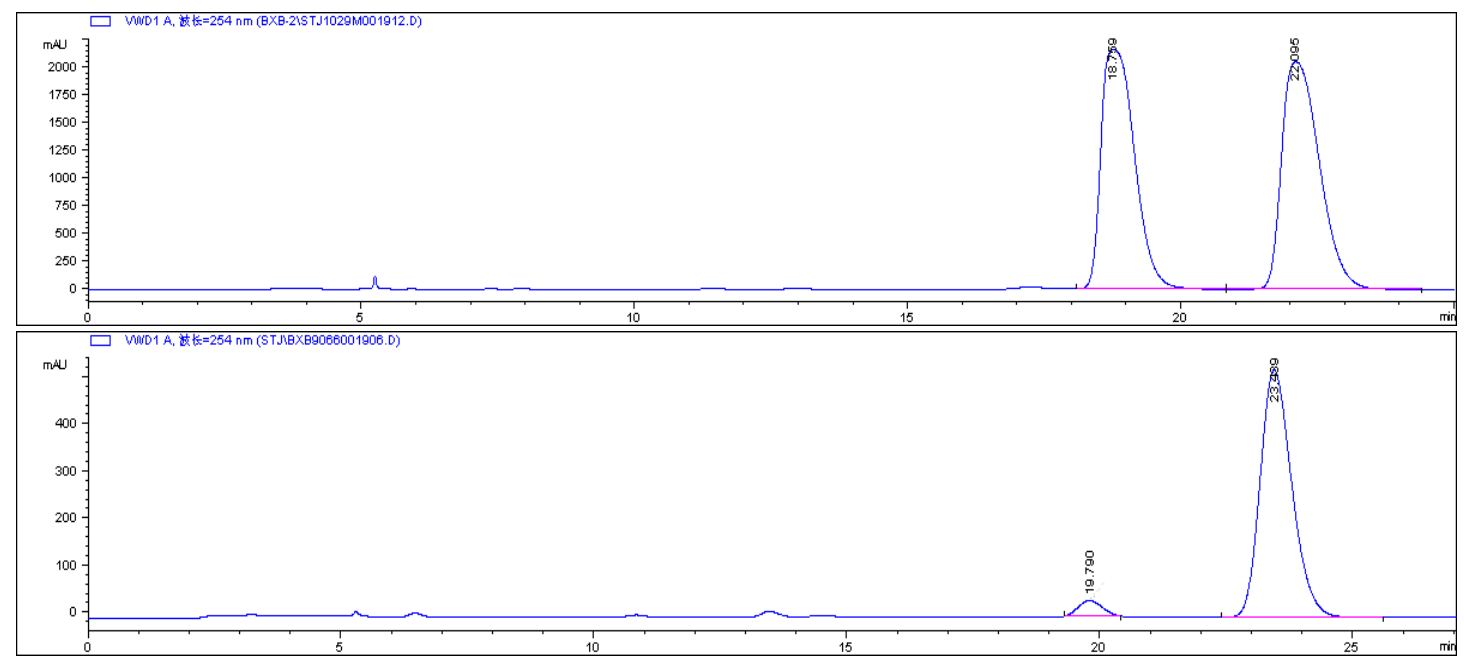

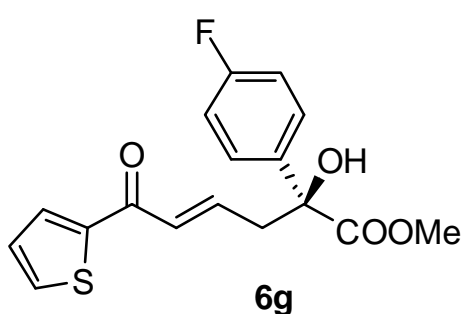

$6 g$

Ketone 5c (18.2 mg, $0.1 \mathrm{mmol}, 1.0$ equiv), $\mathrm{K}_{2} \mathrm{HPO}_{4}(34.8 \mathrm{mg}$, $0.2 \mathrm{mmol}, 2.0$ equiv) and catalyst IV (4.4 $\mathrm{mg}, 0.01 \mathrm{mmol}, 0.1$ equiv) were dissolved in tert-butylbenzene $(0.8 \mathrm{~mL})$ and stirred at $-20{ }^{\circ} \mathrm{C}$ for $10 \mathrm{~min}$. Then allyl ketone $1 \mathbf{y}(45.7 \mathrm{mg}$, $0.3 \mathrm{mmol}, 3.0$ equiv) was added. The reaction mixture was stirred at $-20{ }^{\circ} \mathrm{C}$ and monitored by TLC. Upon complete consumption of ketone 5c, the reaction mixture was directly loaded onto a short silica gel column, followed by gradient elution with PE/EA mixture (20/1-5/1 ratio). Removing the solvent in vacuo, afforded product 6g. Colorless oil; $29.4 \mathrm{mg}(0.1 \mathrm{mmol}), 88 \%$ yield; $94 \% e e ;[\alpha]_{\mathrm{D}}^{26} 7.1\left(\right.$ ( $\left.1.56, \mathrm{CHCl}_{3}\right)$; ${ }^{1} \mathrm{H}$ NMR $\left(300 \mathrm{MHz}, \mathrm{CDCl}_{3}\right) \delta 7.69(\mathrm{dd}, J=3.8,1.0 \mathrm{~Hz}, 1 \mathrm{H}), 7.65(\mathrm{dd}, J=4.9,1.0 \mathrm{~Hz}, 1 \mathrm{H})$, 7.61-7.56 (m, 2H), 7.15-7.12 (m, 1H), 7.08-7.01 (m, 2H), 6.99-6.91 (m, 1H), $6.84(\mathrm{~d}, J=$ $15.4 \mathrm{~Hz}, 1 \mathrm{H}), 3.85(\mathrm{~s}, 1 \mathrm{H}), 3.82(\mathrm{~s}, 3 \mathrm{H}), 3.19-3.11(\mathrm{~m}, 1 \mathrm{H}), 3.01-2.94(\mathrm{~m}, 1 \mathrm{H}) ;{ }^{13} \mathrm{C} \mathrm{NMR}(75$ $\left.\mathrm{MHz}, \mathrm{CDCl}_{3}\right) \delta 181.8,174.3,164.2,160.9,144.8,141.4,136.5,134.0,132.1,129.4,128.2$, 127.3, 127.2, 115.5, 115.2, 77.6, 53.6, 43.0; HRMS (ESI) m/z 357.0581 $\left(\mathrm{M}+\mathrm{H}^{+}\right)$, calc. for $\mathrm{C}_{17} \mathrm{H}_{15} \mathrm{FO}_{4} \mathrm{SNa} 357.0573$.

The ee was determined by HPLC analysis. CHIRALPAK IE (4.6 mm i.d. x $250 \mathrm{~mm}$ ); Hexane/2-propanol $=80 / 20$; flow rate $1.0 \mathrm{~mL} / \mathrm{min} ; 25^{\circ} \mathrm{C} ; 254 \mathrm{~nm}$; retention time: $23.4 \mathrm{~min}$ (minor) and 29.4min (major).

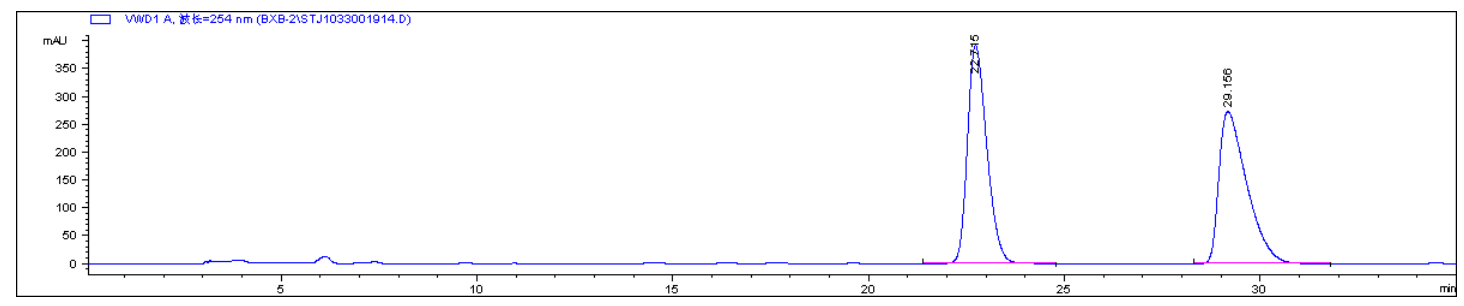



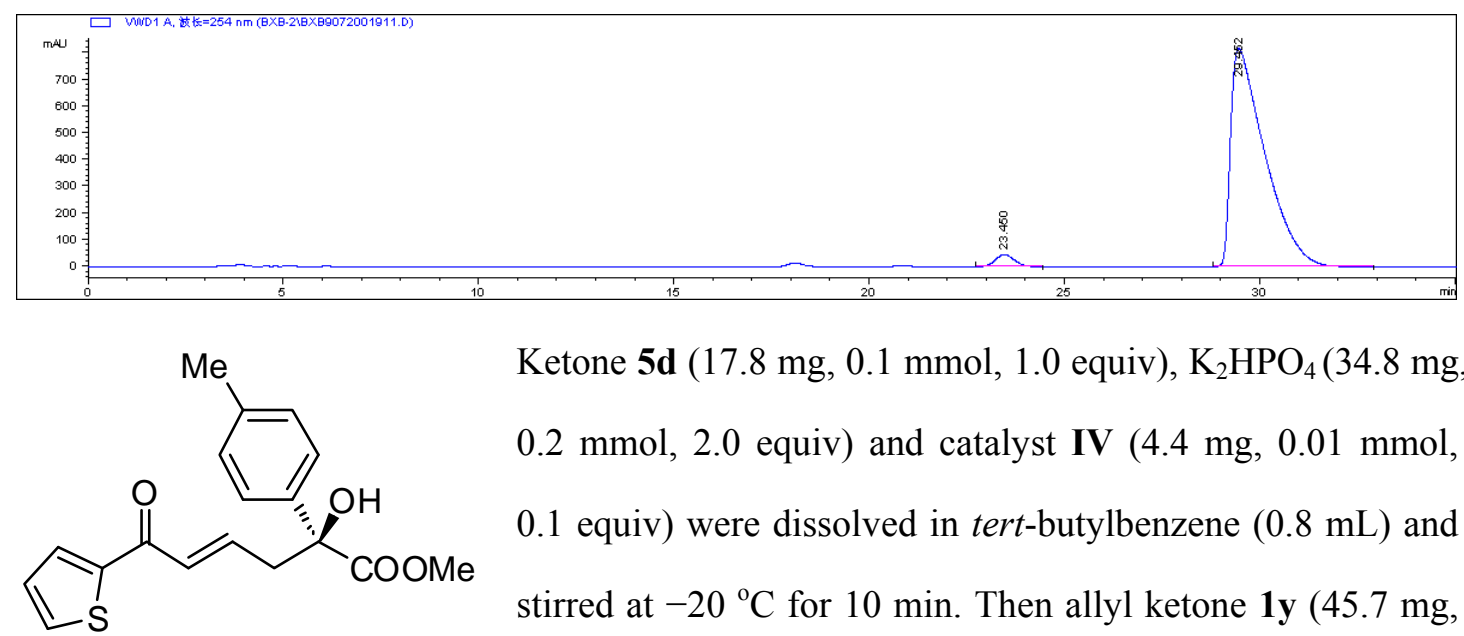

Ketone 5d (17.8 mg, 0.1 mmol, 1.0 equiv), $\mathrm{K}_{2} \mathrm{HPO}_{4}(34.8 \mathrm{mg}$, $0.2 \mathrm{mmol}, 2.0$ equiv) and catalyst $\mathbf{I V}(4.4 \mathrm{mg}, 0.01 \mathrm{mmol}$, 0.1 equiv) were dissolved in tert-butylbenzene $(0.8 \mathrm{~mL})$ and stirred at $-20{ }^{\circ} \mathrm{C}$ for $10 \mathrm{~min}$. Then allyl ketone $1 \mathbf{y}(45.7 \mathrm{mg}$,

6h 0.3 mmol, 3.0 equiv) was added. The reaction mixture was stirred at $-20{ }^{\circ} \mathrm{C}$ and monitored by TLC. Upon complete consumption of ketone 5d, the reaction mixture was directly loaded onto a short silica gel column, followed by gradient elution with PE/EA mixture (20/1-5/1 ratio). Removing the solvent in vacuo, afforded product 6h. Colorless oil; $26.8 \mathrm{mg}(0.1 \mathrm{mmol}), 81 \%$ yield; $96 \% e e ;[\alpha]_{\mathrm{D}}^{26}-6.5\left(c 1.13, \mathrm{CHCl}_{3}\right)$; ${ }^{1} \mathrm{H}$ NMR $\left(300 \mathrm{MHz}, \mathrm{CDCl}_{3}\right) \delta$ 7.70-7.68 (m, 1H), $7.64(\mathrm{~d}, J=4.9 \mathrm{~Hz}, 1 \mathrm{H}), 7.48(\mathrm{~d}, J=8.2$ $\mathrm{Hz}, 2 \mathrm{H}), 7.18(\mathrm{~d}, J=8.1 \mathrm{~Hz}, 2 \mathrm{H}), 7.14-7.11(\mathrm{~m}, 1 \mathrm{H}), 7.04-6.94(\mathrm{~m}, 1 \mathrm{H}), 6.85$ (d, $J=15.4 \mathrm{~Hz}$, 1H), $3.80(\mathrm{~s}, 3 \mathrm{H}), 3.77(\mathrm{~s}, 1 \mathrm{H}), 3.20-3.14(\mathrm{~m}, 1 \mathrm{H}), 3.03-2.96(\mathrm{~m}, 1 \mathrm{H}), 2.34(\mathrm{~s}, 3 \mathrm{H}) ;{ }^{13} \mathrm{C}$ NMR $\left(75 \mathrm{MHz}, \mathrm{CDCl}_{3}\right) \delta 182.0,174.3,144.8,141.9,137.9,133.9,132.1,129.2,128.1,125.2,77.9$, 53.4, 42.7, 21.0; HRMS (ESI) m/z 353.0824 (M+Na ${ }^{+}$, calc. for $\mathrm{C}_{18} \mathrm{H}_{18} \mathrm{O}_{4} \mathrm{SNa} 353.0823$.

The ee was determined by HPLC analysis. CHIRALPAK IE (4.6 mm i.d. x $250 \mathrm{~mm}$ ); Hexane $/ 2$-propanol $=80 / 20$; flow rate $1.0 \mathrm{~mL} / \mathrm{min} ; 25^{\circ} \mathrm{C} ; 254 \mathrm{~nm}$; retention time: $41.3 \mathrm{~min}$ (minor) and 42.5 min (major).
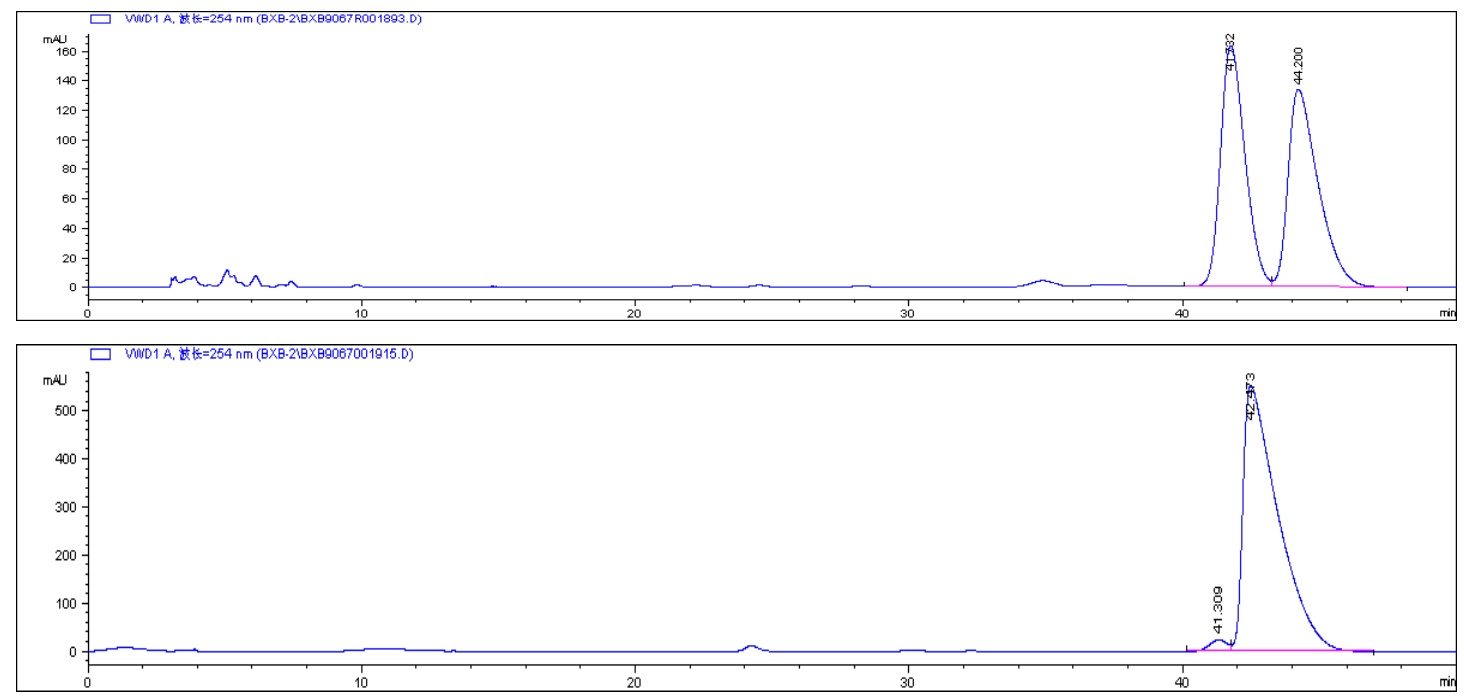


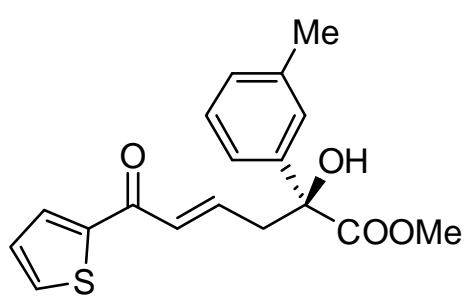

Ketone 5e (17.8 mg, $0.1 \mathrm{mmol}, 1.0$ equiv), $\mathrm{K}_{2} \mathrm{HPO}_{4}(34.8 \mathrm{mg}$, $0.2 \mathrm{mmol}, 2.0$ equiv) and catalyst IV (4.4 $\mathrm{mg}, 0.01 \mathrm{mmol}, 0.1$ equiv) were dissolved in tert-butylbenzene $(0.8 \mathrm{~mL})$ and stirred at $-20{ }^{\circ} \mathrm{C}$ for $10 \mathrm{~min}$. Then allyl ketone $\mathbf{1 y}(45.7 \mathrm{mg}$,

6i 0.3 mmol, 3.0 equiv) was added. The reaction mixture was stirred at $-20{ }^{\circ} \mathrm{C}$ and monitored by TLC. Upon complete consumption of ketone 5e, the reaction mixture was directly loaded onto a short silica gel column, followed by gradient elution with PE/EA mixture (20/1-5/1 ratio). Removing the solvent in vacuo, afforded product 6i. Colorless oil; $27.1 \mathrm{mg}(0.1 \mathrm{mmol}), 82 \%$ yield; $96 \% e e$; $[\alpha]_{\mathrm{D}}^{26}-4.8\left(\right.$ c 1.28, $\left.\mathrm{CHCl}_{3}\right)$; ${ }^{1} \mathrm{H}$ NMR $\left(300 \mathrm{MHz}, \mathrm{CDCl}_{3}\right) \delta 7.69(\mathrm{~d}, J=3.0 \mathrm{~Hz}, 1 \mathrm{H}), 7.64(\mathrm{~d}, J=4.9 \mathrm{~Hz}, 1 \mathrm{H}), 7.39$ (d, $J=$ $12.2 \mathrm{~Hz}, 2 \mathrm{H}), 7.26(\mathrm{t}, \mathrm{J}=7.61 \mathrm{~Hz}, 1 \mathrm{H}), 7.14-7.12(\mathrm{~m}, 2 \mathrm{H}), 7.04-6.94(\mathrm{~m}, 1 \mathrm{H}), 6.85(\mathrm{~d}, J=$ $15.4 \mathrm{~Hz}, 1 \mathrm{H}), 3.81(\mathrm{~s}, 3 \mathrm{H}), 3.77(\mathrm{~s}, 1 \mathrm{H}), 3.22-3.14(\mathrm{~m}, 1 \mathrm{H}), 3.04-2.96(\mathrm{~m}, 1 \mathrm{H}), 2.37(\mathrm{~s}, 3 \mathrm{H})$; ${ }^{13} \mathrm{C}$ NMR $\left(75 \mathrm{MHz}, \mathrm{CDCl}_{3}\right) \delta 182.0,174.5,144.9,141.9,140.8,138.3,133.9,132.2,129.3$, 129.0, 128.4, 128.2, 125.9, 122.4, 78.0, 53.5, 42.8, 21.6; HRMS (ESI) m/z $353.0823\left(\mathrm{M}+\mathrm{Na}^{+}\right)$, calc. for $\mathrm{C}_{18} \mathrm{H}_{18} \mathrm{O}_{4} \mathrm{SNa} 353.0824$.

The ee was determined by HPLC analysis. CHIRALPAK IE $(4.6 \mathrm{~mm}$ i.d. x $250 \mathrm{~mm})$; Hexane/2-propanol $=80 / 20$; flow rate $1.0 \mathrm{~mL} / \mathrm{min} ; 25{ }^{\circ} \mathrm{C} ; 254 \mathrm{~nm}$; retention time: $32.7 \mathrm{~min}$ (major) and $35.7 \mathrm{~min}$ (minor).
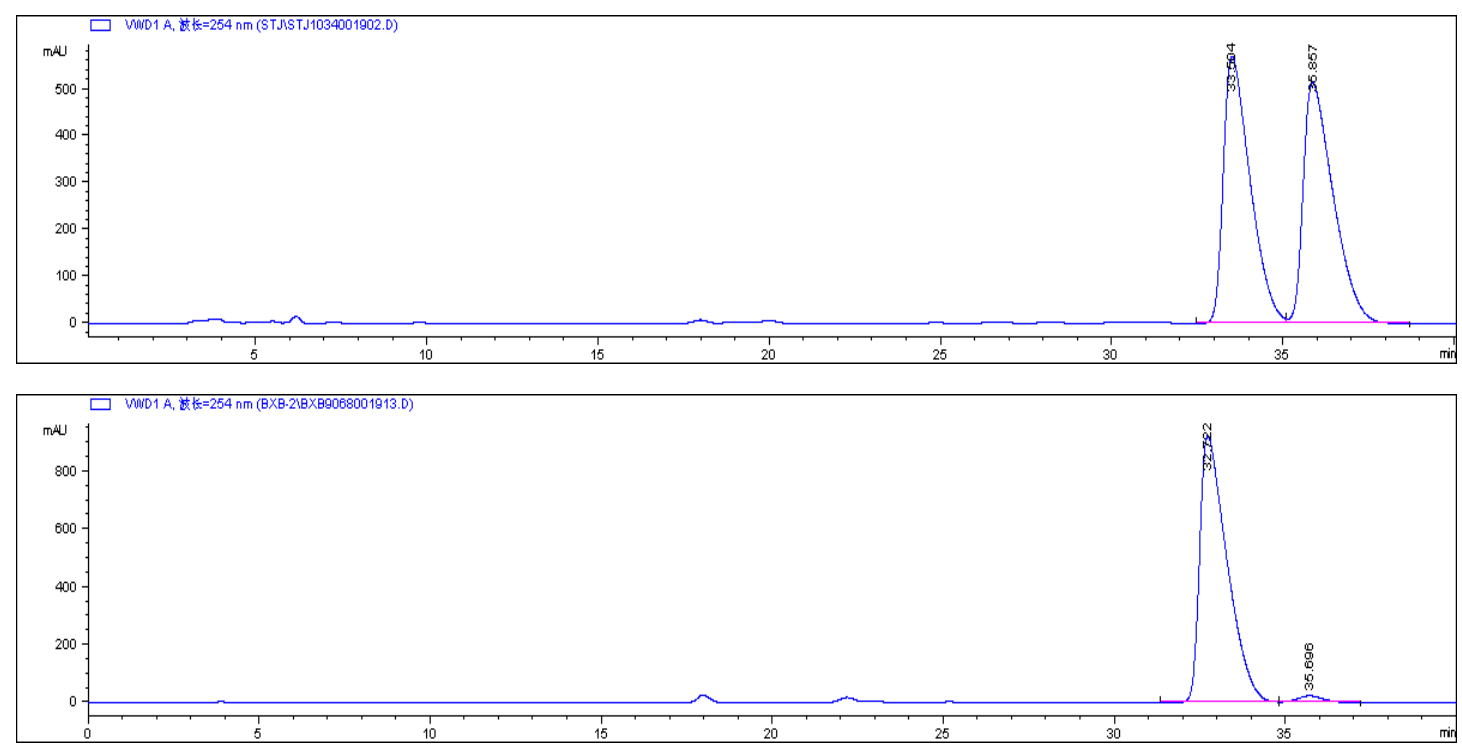


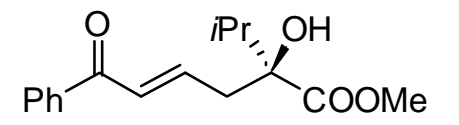

Ketone $5 \mathbf{f}$ (13.0 mg, $0.1 \mathrm{mmol}, 1.0$ equiv), $\mathrm{K}_{2} \mathrm{HPO}_{4}(34.8 \mathrm{mg}$, $0.2 \mathrm{mmol}, 2.0$ equiv) and catalyst $\mathbf{I V}(4.4 \mathrm{mg}, 0.01 \mathrm{mmol}, 0.1$

6j equiv) were dissolved in tert-butylbenzene $(0.8 \mathrm{~mL})$ and stirred at $-20{ }^{\circ} \mathrm{C}$ for $10 \mathrm{~min}$. Then allyl ketone $1 \mathrm{a}(43.9 \mathrm{mg}, 0.3 \mathrm{mmol}$, 3.0 equiv) was added. The reaction mixture was stirred at $-20{ }^{\circ} \mathrm{C}$ and monitored by TLC. Upon complete consumption of ketone $\mathbf{5 f}$, the reaction mixture was directly loaded onto a short silica gel column, followed by gradient elution with PE/EA mixture (20/1-5/1 ratio). Removing the solvent in vacuo, afforded product $\mathbf{6 j}$. Colorless oil; $24.0 \mathrm{mg}(0.1 \mathrm{mmol}), 87 \%$ yield; 93\% ee; $[\alpha]_{\mathrm{D}}^{26}+7.5\left(c 0.53, \mathrm{CHCl}_{3}\right) ;{ }^{1} \mathrm{H} \mathrm{NMR}\left(300 \mathrm{MHz}, \mathrm{CDCl}_{3}\right) \delta 7.90-7.87(\mathrm{~m}, 2 \mathrm{H})$, $7.55(\mathrm{t}, J=7.3 \mathrm{~Hz}, 1 \mathrm{H}), 7.46(\mathrm{t}, J=7.5 \mathrm{~Hz}, 2 \mathrm{H}), 6.91-6.88(\mathrm{~m}, 2 \mathrm{H}), 3.79(\mathrm{~s}, 3 \mathrm{H}), 3.24(\mathrm{~s}, 1 \mathrm{H})$, 2.79-2.73 (m, 1H), 2.68-2.61 (m, 1H), 2.07-1.96 (m, 1H), $1.00(\mathrm{~d}, J=6.8 \mathrm{~Hz}, 3 \mathrm{H}), 0.87(\mathrm{~d}$, $J=6.8 \mathrm{~Hz}, 3 \mathrm{H}) ;{ }^{13} \mathrm{C} \mathrm{NMR}\left(75 \mathrm{MHz}, \mathrm{CDCl}_{3}\right) \delta 190.7,176.3,143.5,137.6,132.8,129.4,128.6$ (two peaks), 79.8, 52.9, 40.3, 35.5, 17.4, 15.9; HRMS (ESI) m/z 277.1438 $\left(\mathrm{M}+\mathrm{H}^{+}\right)$, calc. for $\mathrm{C}_{16} \mathrm{H}_{21} \mathrm{O}_{4} 277.1440$.

The ee was determined by HPLC analysis. CHIRALPAK IC (4.6 mm i.d. x $250 \mathrm{~mm}$ ); Hexane/2-propanol $=80 / 20$; flow rate $1.0 \mathrm{~mL} / \mathrm{min} ; 25^{\circ} \mathrm{C} ; 254 \mathrm{~nm}$; retention time: $20.4 \mathrm{~min}$ (minor) and 22.7 min (major).
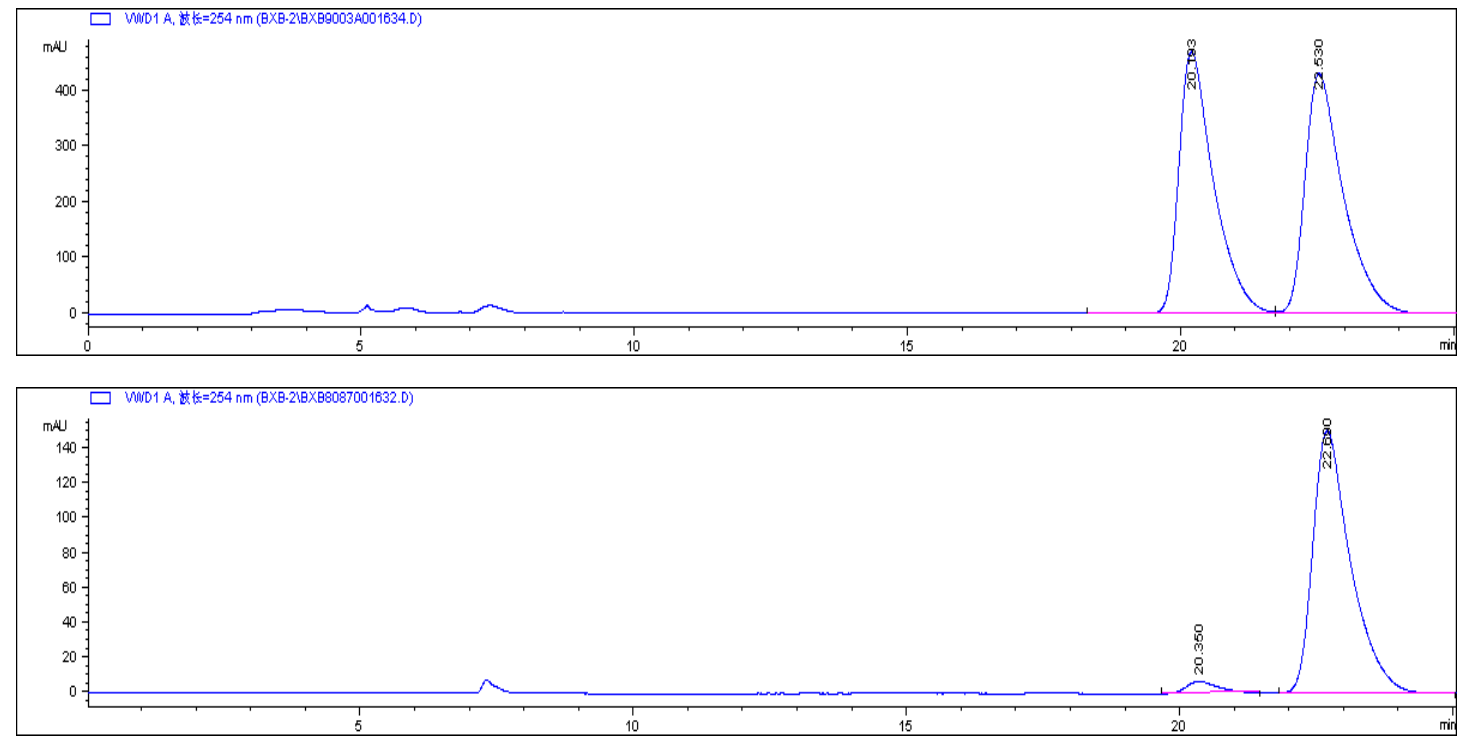

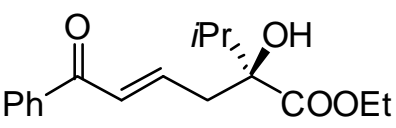

$6 \mathrm{k}$
Ketone 5g (14.4 mg, 0.1 mmol, 1.0 equiv), $\mathrm{K}_{2} \mathrm{HPO}_{4}(34.8 \mathrm{mg}, 0.2$ mmol, 2.0 equiv) and catalyst $\mathbf{I V}$ ( $4.4 \mathrm{mg}, 0.01 \mathrm{mmol}, 0.1$ equiv) were dissolved in tert-butylbenzene $(0.8 \mathrm{~mL})$ and stirred at -20 
${ }^{\circ} \mathrm{C}$ for $10 \mathrm{~min}$. Then allyl ketone $1 \mathrm{a}(43.9 \mathrm{mg}, 0.3 \mathrm{mmol}, 3.0$ equiv) was added. The reaction mixture was stirred at $-20{ }^{\circ} \mathrm{C}$ and monitored by TLC. Upon complete consumption of ketone 5g, the reaction mixture was directly loaded onto a short silica gel column, followed by gradient elution with PE/EA mixture (20/1-5/1 ratio). Removing the solvent in vacuo, afforded product 6k. Colorless oil; $26.7 \mathrm{mg}(0.1 \mathrm{mmol}), 92 \%$ yield; $91 \% e e ;[\alpha]_{\mathrm{D}}^{26}+11.0(c$ 1.28, $\left.\mathrm{CHCl}_{3}\right) ;{ }^{1} \mathrm{H} \mathrm{NMR}\left(300 \mathrm{MHz}, \mathrm{CDCl}_{3}\right) \delta 7.89-7.86(\mathrm{~m}, 2 \mathrm{H}), 7.57-7.52(\mathrm{~m}, 1 \mathrm{H}), 7.45(\mathrm{t}, J$ $=7.4 \mathrm{~Hz}, 2 \mathrm{H}), 6.96-6.84(\mathrm{~m}, 2 \mathrm{H}), 4.24(\mathrm{q}, J=7.1 \mathrm{~Hz}, 2 \mathrm{H}), 3.28(\mathrm{~s}, 1 \mathrm{H}), 2.79-2.72(\mathrm{~m}, 1 \mathrm{H})$, 2.68-2.61 (m, 1H), 2.08-1.99 (m, 1H), 1.28 (t, $J=7.1 \mathrm{~Hz}, 3 \mathrm{H}), 1.00(\mathrm{~d}, J=6.8 \mathrm{~Hz}, 3 \mathrm{H}), 0.87$ $(\mathrm{d}, J=6.8 \mathrm{~Hz}, 3 \mathrm{H}) ;{ }^{13} \mathrm{C}$ NMR $\left(75 \mathrm{MHz}, \mathrm{CDCl}_{3}\right) \delta 190.7,175.7,143.6,137.6,132.7,129.4$, 128.6, 128.5, 79.3, 62.2, 40.2, 35.3, 17.2, 15.9, 14.3; HRMS (ESI) m/z $313.1417\left(\mathrm{M}+\mathrm{Na}^{+}\right)$, calc. for $\mathrm{C}_{17} \mathrm{H}_{22} \mathrm{O}_{4} \mathrm{Na} 313.1416$.

The ee was determined by HPLC analysis. CHIRALPAK IC (4.6 mm i.d. x $250 \mathrm{~mm}$ ); Hexane/2-propanol $=80 / 20$; flow rate $1.0 \mathrm{~mL} / \mathrm{min} ; 25{ }^{\circ} \mathrm{C} ; 254 \mathrm{~nm}$; retention time: $17.2 \mathrm{~min}$ (minor) and 21.4min (major).
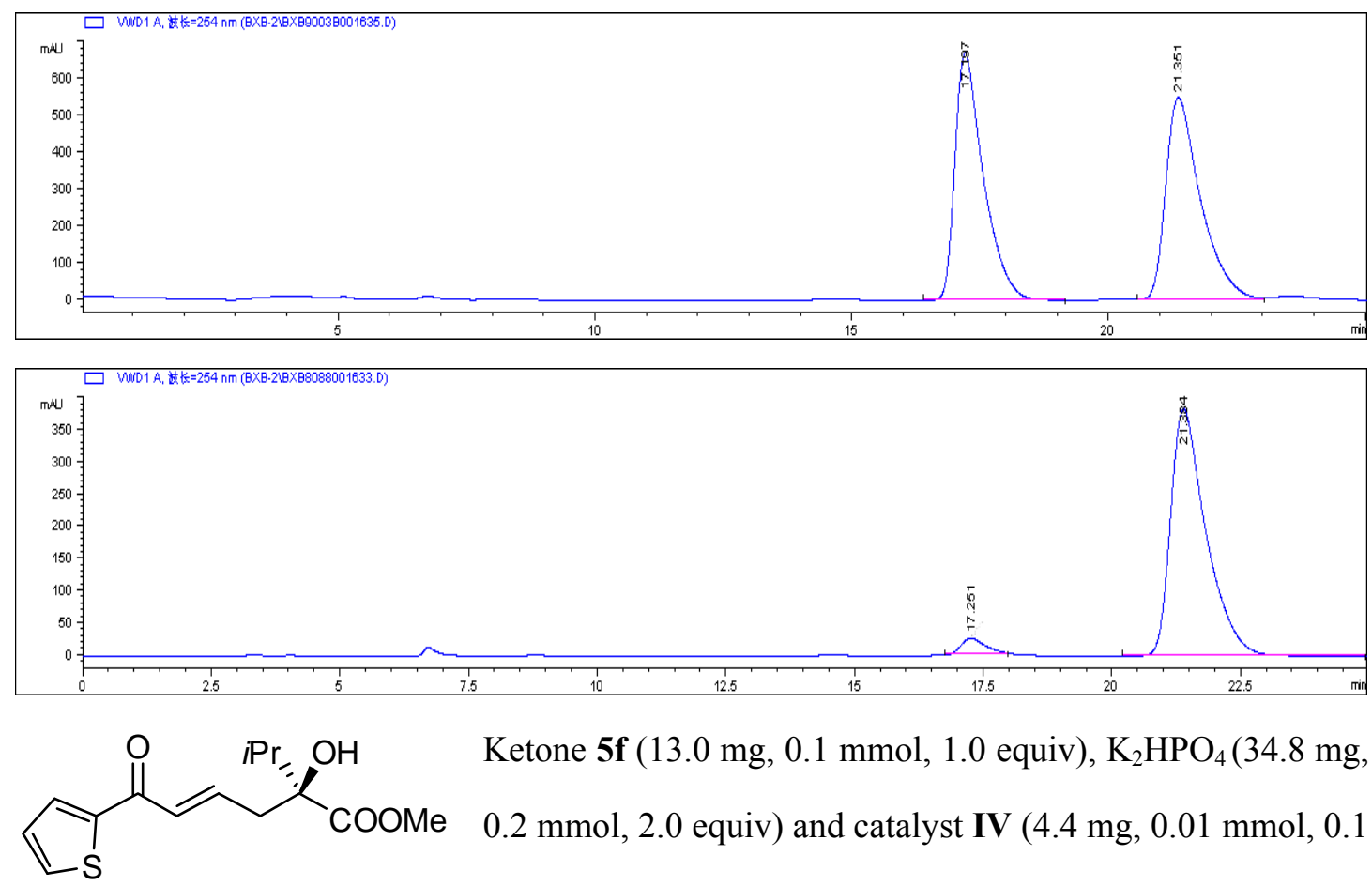

Ketone $5 \mathbf{f}$ (13.0 mg, $0.1 \mathrm{mmol}, 1.0$ equiv), $\mathrm{K}_{2} \mathrm{HPO}_{4}(34.8 \mathrm{mg}$, $0.2 \mathrm{mmol}, 2.0$ equiv) and catalyst $\mathbf{I V}(4.4 \mathrm{mg}, 0.01 \mathrm{mmol}, 0.1$ 6l equiv) were dissolved in tert-butylbenzene $(0.8 \mathrm{~mL})$ and stirred at $-20{ }^{\circ} \mathrm{C}$ for $10 \mathrm{~min}$. Then allyl ketone $1 \mathbf{y}(45.7 \mathrm{mg}$, $0.3 \mathrm{mmol}, 3.0$ equiv) was added. The reaction mixture was stirred at $-20{ }^{\circ} \mathrm{C}$ and monitored by TLC. Upon complete consumption of ketone $\mathbf{5 f}$, the reaction mixture was directly loaded onto 
a short silica gel column, followed by gradient elution with PE/EA mixture (20/1-5/1 ratio). Removing the solvent in vacuo, afforded product 61 . Colorless oil; $27.1 \mathrm{mg}(0.1 \mathrm{mmol}), 71 \%$ yield; $97 \% e e ;[\alpha]_{\mathrm{D}}^{26}+5.8\left(c 0.83, \mathrm{CHCl}_{3}\right) ;{ }^{1} \mathrm{H} \mathrm{NMR}\left(300 \mathrm{MHz}, \mathrm{CDCl}_{3}\right) \delta 7.73(\mathrm{dd}, J=3.8,0.9$ $\mathrm{Hz}, 1 \mathrm{H}), 7.65(\mathrm{dd}, J=4.9,0.9 \mathrm{~Hz}, 1 \mathrm{H}), 7.16-7.13(\mathrm{~m}, 1 \mathrm{H}), 7.02-6.92(\mathrm{~m}, 1 \mathrm{H}), 6.82(\mathrm{~d}, J=$ 15.4 Hz, 1H), $3.78(\mathrm{~s}, 3 \mathrm{H}), 3.24(\mathrm{~s}, 1 \mathrm{H}), 2.79-2.72(\mathrm{~m}, 1 \mathrm{H}), 2.67-2.60(\mathrm{~m}, 1 \mathrm{H}), 2.09-1.96(\mathrm{~m}$, $1 \mathrm{H}), 1.00(\mathrm{~d}, J=6.8 \mathrm{~Hz}, 3 \mathrm{H}), 0.86(\mathrm{~d}, J=6.8 \mathrm{~Hz}, 3 \mathrm{H}) ;{ }^{13} \mathrm{C} \mathrm{NMR}\left(75 \mathrm{MHz}, \mathrm{CDCl}_{3}\right) \delta 182.0$, 176.2, 144.8, 142.6, 133.9, 132.1, 128.8, 128.2, 79.7, 52.9, 40.0, 35.4, 17.4, 15.9; HRMS (ESI) $\mathrm{m} / \mathrm{z} 305.0823\left(\mathrm{M}+\mathrm{Na}^{+}\right)$, calc. for $\mathrm{C}_{14} \mathrm{H}_{18} \mathrm{O}_{4} \mathrm{SNa} 305.0824$.

The ee was determined by HPLC analysis. CHIRALPAK IE (4.6 mm i.d. x $250 \mathrm{~mm}$ ); Hexane/2-propanol $=70 / 30$; flow rate $1.0 \mathrm{~mL} / \mathrm{min} ; 25^{\circ} \mathrm{C} ; 254 \mathrm{~nm}$; retention time: $20.3 \mathrm{~min}$ (minor) and 34.9 min (major).

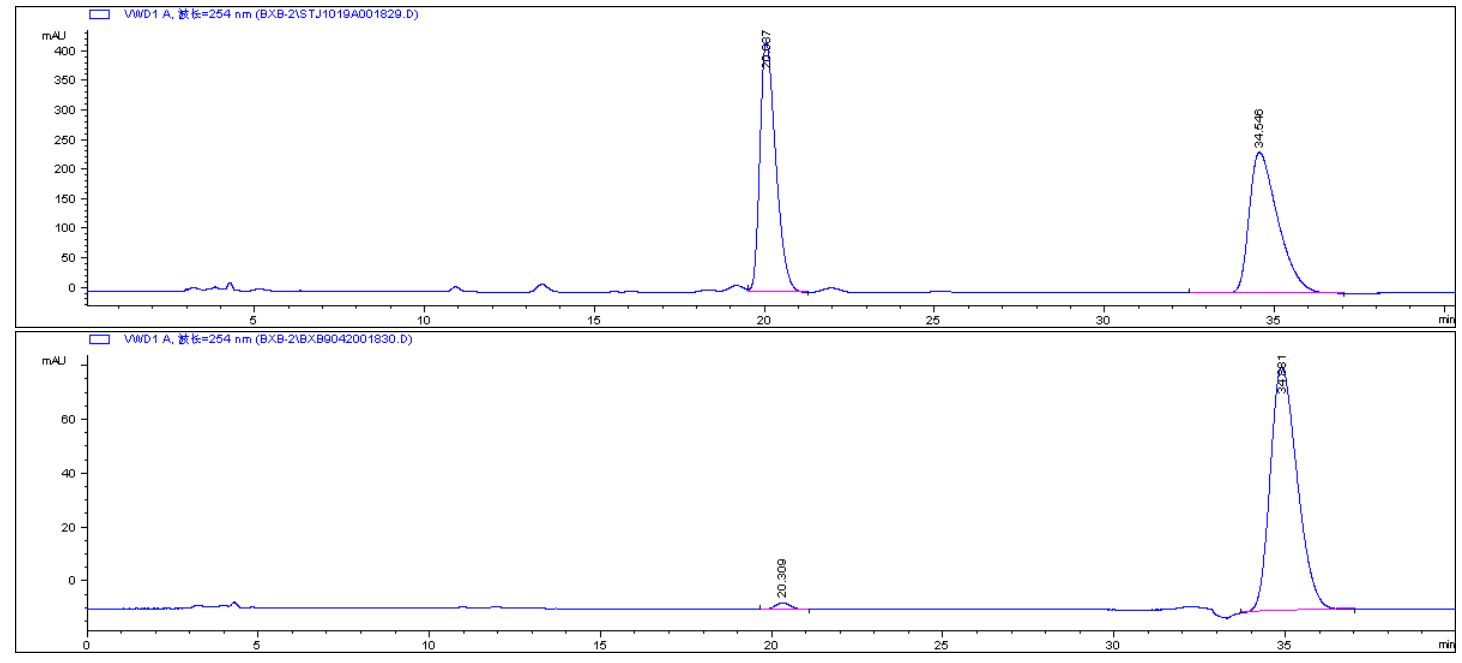

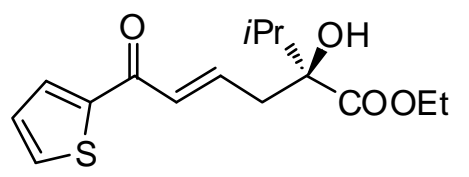

$6 \mathrm{~m}$
Ketone 5g (14.4 mg, $0.1 \mathrm{mmol}, 1.0$ equiv), $\mathrm{K}_{2} \mathrm{HPO}_{4}(34.8 \mathrm{mg}$, $0.2 \mathrm{mmol}, 2.0$ equiv) and catalyst IV ( $4.4 \mathrm{mg}, 0.01 \mathrm{mmol}, 0.1$ equiv) were dissolved in tert-butylbenzene $(0.8 \mathrm{~mL})$ and stirred at $-20{ }^{\circ} \mathrm{C}$ for $10 \mathrm{~min}$. Then allyl ketone $\mathbf{1 y}(45.7 \mathrm{mg}$,

$0.3 \mathrm{mmol}, 3.0$ equiv) was added. The reaction mixture was stirred at $-20{ }^{\circ} \mathrm{C}$ and monitored by TLC. Upon complete consumption of ketone $\mathbf{5 g}$, the reaction mixture was directly loaded onto a short silica gel column, followed by gradient elution with PE/EA mixture (20/1-5/1 ratio). Removing the solvent in vacuo, afforded product $\mathbf{6 m}$. Colorless oil; $26.4 \mathrm{mg}(0.1$ mmol), $89 \%$ yield; $97 \% e e ;[\alpha]_{\mathrm{D}}^{26}+8.7\left(c 1.61, \mathrm{CHCl}_{3}\right) ;{ }^{1} \mathrm{H}$ NMR $\left(300 \mathrm{MHz}, \mathrm{CDCl}_{3}\right) \delta 7.73$ $(\mathrm{dd}, J=3.8,1.0 \mathrm{~Hz}, 1 \mathrm{H}), 7.65(\mathrm{dd}, J=4.9,1.0 \mathrm{~Hz}, 1 \mathrm{H}), 7.15-7.12(\mathrm{~m}, 1 \mathrm{H}), 7.03-6.93(\mathrm{~m}$, 1H), $6.81(\mathrm{~d}, J=15.3 \mathrm{~Hz}, 1 \mathrm{H}), 4.25(\mathrm{q}, J=7.1 \mathrm{~Hz}, 2 \mathrm{H}), 3.28(\mathrm{~s}, 1 \mathrm{H}), 2.78-2.71(\mathrm{~m}, 1 \mathrm{H})$, 
2.68-2.60 (m, 1H), 2.10-1.96 (m, 1H), $1.29(\mathrm{t}, J=7.1 \mathrm{~Hz}, 3 \mathrm{H}), 1.00(\mathrm{~d}, J=6.8 \mathrm{~Hz}, 3 \mathrm{H}), 0.87$ $(\mathrm{d}, J=6.8 \mathrm{~Hz}, 3 \mathrm{H}) ;{ }^{13} \mathrm{C}$ NMR $\left(75 \mathrm{MHz}, \mathrm{CDCl}_{3}\right) \delta 182.0,175.7,144.8,142.7,133.9,132.0$, 128.7, 128.2, 79.3, 62.2, 40.1, 35.4, 17.2, 15.9, 14.3; HRMS (ESI) m/z $319.0979\left(\mathrm{M}+\mathrm{Na}^{+}\right)$, calc. for $\mathrm{C}_{15} \mathrm{H}_{20} \mathrm{O}_{4} \mathrm{SNa} 319.0980$.

The ee was determined by HPLC analysis. CHIRALPAK IE $(4.6 \mathrm{~mm}$ i.d. x $250 \mathrm{~mm}$ ); Hexane $/ 2$-propanol $=70 / 30 ;$ flow rate $1.0 \mathrm{~mL} / \mathrm{min} ; 25{ }^{\circ} \mathrm{C} ; 254 \mathrm{~nm}$; retention time: $17.7 \mathrm{~min}$ (minor) and 29.7 min (major).
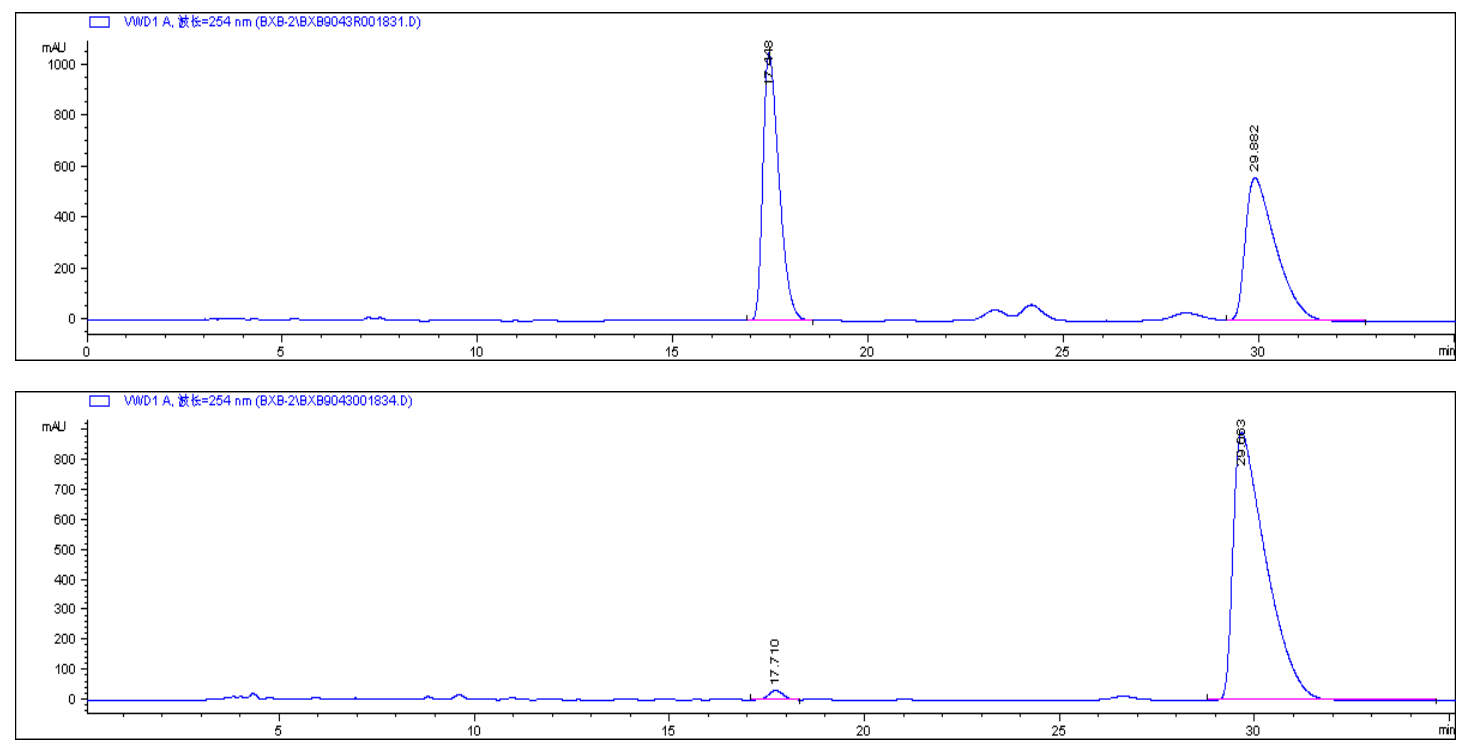<smiles>CCOP(=O)(OCC)C(O)(C/C=C/C(=O)c1ccccc1)c1ccccc1</smiles>

Ketone 5h (24.2 mg, 0.1 mmol, 1.0 equiv), $\mathrm{K}_{2} \mathrm{HPO}_{4}(34.8 \mathrm{mg}, 0.2$ mmol, 2.0 equiv) and catalyst IV (4.4 mg, $0.01 \mathrm{mmol}, 0.1$ equiv) were dissolved in tert-butylbenzene $(0.8 \mathrm{~mL})$ and stirred at -20

6n ${ }^{\circ} \mathrm{C}$ for $10 \mathrm{~min}$. Then allyl ketone 1a $(43.9 \mathrm{mg}, 0.3 \mathrm{mmol}, 3.0$ equiv) was added. The reaction mixture was stirred at $-20{ }^{\circ} \mathrm{C}$ and monitored by TLC. Upon complete consumption of ketone $\mathbf{5} \mathbf{h}$, the reaction mixture was directly loaded onto a short silica gel column, followed by gradient elution with PE/EA mixture (20/1-5/1 ratio). Removing the solvent in vacuo, afforded product $6 \mathbf{n}$. White solid; Mp $130.1-131.9{ }^{\circ} \mathrm{C} ; 24.1$ mg (0.1 mmol), 62\% yield; 92\% ee; $[\alpha]_{\mathrm{D}}^{26}-16.1\left(c \quad 1.13, \mathrm{CHCl}_{3}\right) ;{ }^{1} \mathrm{H} \mathrm{NMR}\left(300 \mathrm{MHz}, \mathrm{CDCl}_{3}\right)$ $\delta 7.73-7.70(\mathrm{~m}, 2 \mathrm{H}), 7.76-7.59(\mathrm{~m}, 2 \mathrm{H}), 7.50(\mathrm{t}, J=7.4 \mathrm{~Hz}, 1 \mathrm{H}), 7.38(\mathrm{t}, J=7.5 \mathrm{~Hz}, 4 \mathrm{H})$, $7.31(\mathrm{dd}, J=7.2,1.6 \mathrm{~Hz}, 1 \mathrm{H}), 6.86-6.70(\mathrm{~m}, 2 \mathrm{H}), 4.17-4.07(\mathrm{~m}, 2 \mathrm{H}), 4.04-3.89(\mathrm{~m}, 1 \mathrm{H})$, $3.87-3.76(\mathrm{~m}, 1 \mathrm{H}), 3.63(\mathrm{~d}, J=6.7 \mathrm{~Hz}, 1 \mathrm{H}), 3.21(\mathrm{t}, J=7.1 \mathrm{~Hz}, 2 \mathrm{H}), 3.21(\mathrm{t}, J=7.1 \mathrm{~Hz}, 2 \mathrm{H})$, $1.27(\mathrm{t}, J=7.0 \mathrm{~Hz}, 3 \mathrm{H}), 1.17(\mathrm{t}, J=7.1 \mathrm{~Hz}, 3 \mathrm{H}) ;{ }^{13} \mathrm{C} \mathrm{NMR}\left(75 \mathrm{MHz}, \mathrm{CDCl}_{3}\right) \delta 190.8,142.4$, 
$142.2,138.1,137.5,132.6,130.3,128.6,128.4,128.3,128.2,127.7,127.6,126.1$ (two peaks), 76.6, 74.5, 63.7, 63.6, 63.5, 63.4, 41.4, 41.3, 16.4, 16.3(two peaks), 16.2; HRMS (ESI) m/z $411.1336\left(\mathrm{M}+\mathrm{Na}^{+}\right)$, calc. for $\mathrm{C}_{21} \mathrm{H}_{25} \mathrm{O}_{5} \mathrm{P} 411.1337$

The ee was determined by HPLC analysis. CHIRALPAK IC (4.6 mm i.d. x $250 \mathrm{~mm}$ ); Hexane/2-propanol $=80 / 20$; flow rate $1.0 \mathrm{~mL} / \mathrm{min} ; 25^{\circ} \mathrm{C} ; 254 \mathrm{~nm}$; retention time: $19.2 \mathrm{~min}$ (minor) and $22.4 \min$ (major).
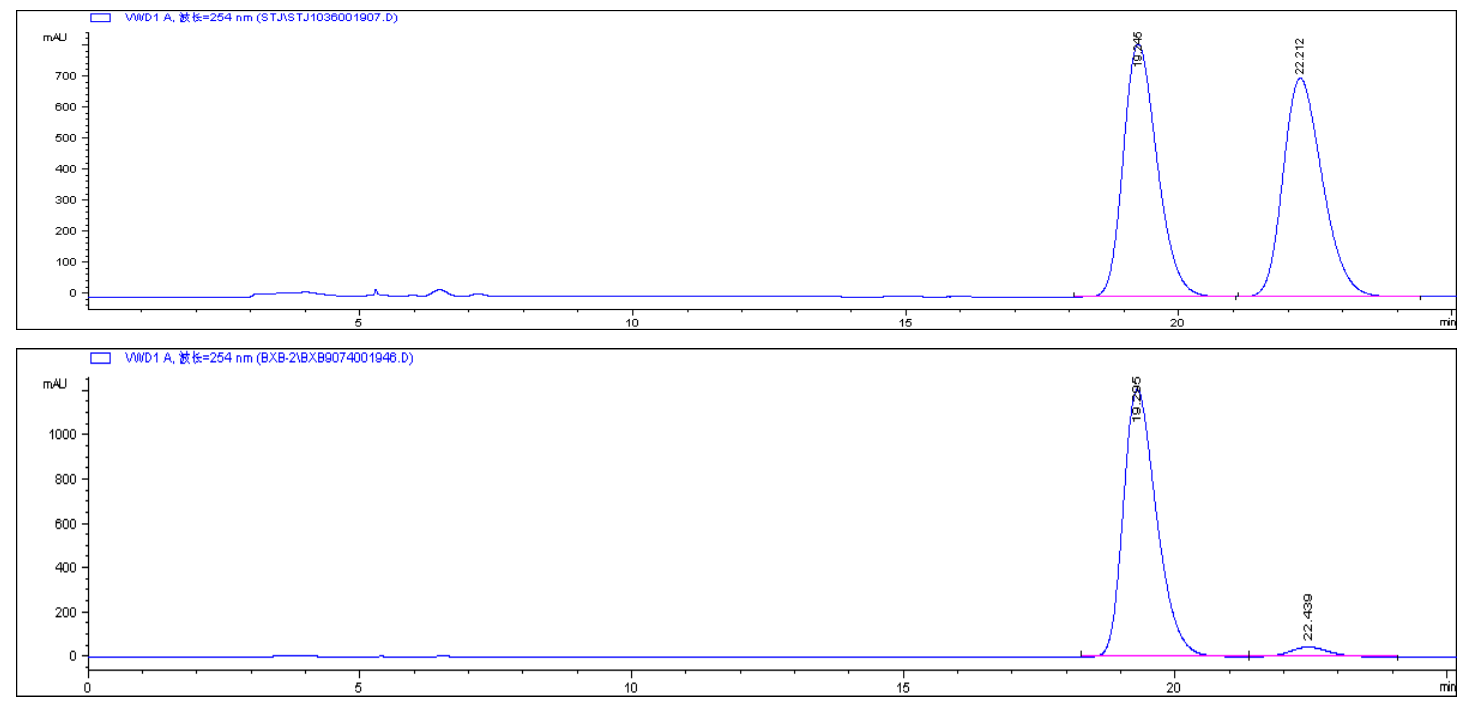

Ketone 5h (24.2 mg, 0.1 mmol, 1.0 equiv), $\mathrm{K}_{2} \mathrm{HPO}_{4}(34.8 \mathrm{mg}$, $0.2 \mathrm{mmol}, 2.0$ equiv) and catalyst IV (4.4 $\mathrm{mg}, 0.01 \mathrm{mmol}, 0.1$ equiv) were dissolved in tert-butylbenzene $(0.8 \mathrm{~mL})$ and stirred at $-20{ }^{\circ} \mathrm{C}$ for $10 \mathrm{~min}$. Then allyl ketone $1 \mathbf{y}(45.7 \mathrm{mg}$, $0.3 \mathrm{mmol}, 3.0$ equiv) was added. The reaction mixture was stirred at $-20{ }^{\circ} \mathrm{C}$ and monitored by TLC. Upon complete consumption of ketone $\mathbf{5 h}$, the reaction mixture was directly loaded onto a short silica gel column, followed by gradient elution with PE/EA mixture (20/1-5/1 ratio). Removing the solvent in vacuo, afforded product 6o. Colorless oil; $36.3 \mathrm{mg}(0.1 \mathrm{mmol})$, 92\% yield; 97\% ee; $[\alpha]_{\mathrm{D}}^{26}-11.3\left(c 0.67, \mathrm{CHCl}_{3}\right) ;{ }^{1} \mathrm{H} \mathrm{NMR}\left(300 \mathrm{MHz}, \mathrm{CDCl}_{3}\right) \delta 7.63-7.57(\mathrm{~m}$, 4H), $7.37(\mathrm{t}, J=7.7 \mathrm{~Hz}, 2 \mathrm{H}), 7.30(\mathrm{dd}, J=7.2,1.6 \mathrm{~Hz}, 1 \mathrm{H}), 7.10-7.07(\mathrm{~m}, 1 \mathrm{H}), 6.91-6.81(\mathrm{~m}$, $1 \mathrm{H}), 6.77(\mathrm{~d}, J=15.5 \mathrm{~Hz}, 1 \mathrm{H}), 4.17-4.07(\mathrm{~m}, 2 \mathrm{H}), 4.04-3.91(\mathrm{~m}, 1 \mathrm{H}), 3.90-3.76(\mathrm{~m}, 1 \mathrm{H})$, $3.50(\mathrm{~d}, J=6.1 \mathrm{~Hz}, 1 \mathrm{H}), 3.21-3.17(\mathrm{~m}, 2 \mathrm{H}), 1.28(\mathrm{t}, J=7.1 \mathrm{~Hz}, 3 \mathrm{H}), 1.17(\mathrm{t}, J=7.1 \mathrm{~Hz}, 3 \mathrm{H})$; ${ }^{13} \mathrm{C}$ NMR $\left(75 \mathrm{MHz}, \mathrm{CDCl}_{3}\right) \delta 181.9,144.8,141.5,141.3,138.0,133.9,132.2,129.6,128.3$ (two peaks), 128.1, 127.8, 127.7, 126.1, 126.0, 76.6, 74.5, 63.8, 63.7, 63.5, 63.4, 41.3, 41.2, 16.4, 16.3 (two peaks), 16.2; HRMS (ESI) $\mathrm{m} / \mathrm{z} 417.0905\left(\mathrm{M}+\mathrm{Na}^{+}\right)$, calc. for $\mathrm{C}_{19} \mathrm{H}_{23} \mathrm{O}_{5} \mathrm{PSNa}$ 
417.0902.

The ee was determined by HPLC analysis. CHIRALPAK IE (4.6 mm i.d. x $250 \mathrm{~mm}$ ); Hexane $/ 2$-propanol $=70 / 30$; flow rate $1.0 \mathrm{~mL} / \mathrm{min} ; 25^{\circ} \mathrm{C} ; 254 \mathrm{~nm}$; retention time: $15.2 \mathrm{~min}$ (minor) and $18.0 \mathrm{~min}$ (major).
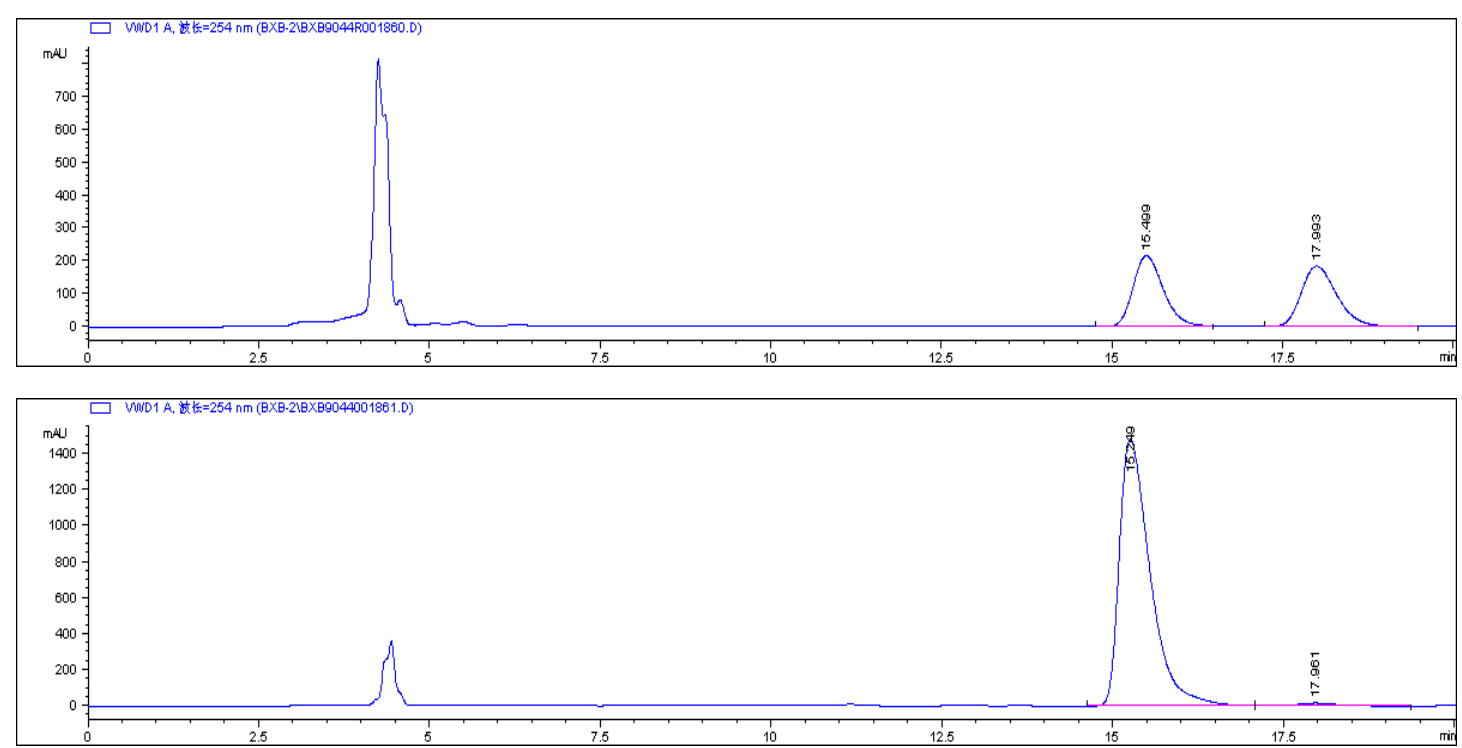

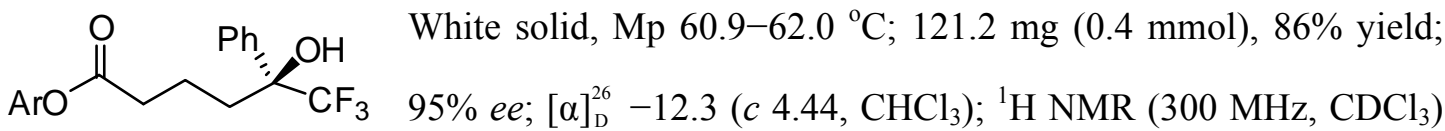
7: $\mathrm{Ar}=4-\mathrm{MeOPh} \quad \delta 7.57(\mathrm{~d}, J=7.5 \mathrm{~Hz}, 2 \mathrm{H}), 7.44-7.34(\mathrm{~m}, 3 \mathrm{H}), 6.98-6.94(\mathrm{~m}, 2 \mathrm{H})$, 6.91-6.87 (m, 2H), $3.80(\mathrm{~s}, 3 \mathrm{H}), 2.94(\mathrm{~s}, 1 \mathrm{H}), 2.56(\mathrm{t}, J=6.9 \mathrm{~Hz}$, $1 \mathrm{H}), 2.45-2.35(\mathrm{~m}, 1 \mathrm{H}), 2.14-2.04(\mathrm{~m}, 1 \mathrm{H}), 1.83-1.68(\mathrm{~m}, 1 \mathrm{H}), 1.56-1.41(\mathrm{~m}, 1 \mathrm{H}) ;{ }^{13} \mathrm{C}$ $\operatorname{NMR}\left(75 \mathrm{MHz}, \mathrm{CDCl}_{3}\right) \delta 172.6,157.3,143.9,136.0,128.5,128.4,126.4,125.6(\mathrm{q}, J=284.1$ Hz), 122.2, 114.5, 76.8 (q, $J=27.9$ Hz), 55.6, 33.9, 33.4, 17.6; HRMS (ESI) m/z 367.1160 $\left(\mathrm{M}+\mathrm{H}^{+}\right)$, calc. for $\mathrm{C}_{19} \mathrm{H}_{18} \mathrm{~F}_{3} \mathrm{O}_{4} 367.1157$.

The ee was determined by HPLC analysis. CHIRALPAK IB (4.6 mm i.d. x $250 \mathrm{~mm}$ ); Hexane $/ 2$-propanol $=90 / 10$; flow rate $1.0 \mathrm{~mL} / \mathrm{min} ; 25{ }^{\circ} \mathrm{C} ; 254 \mathrm{~nm}$; retention time: $10.7 \mathrm{~min}$ (minor) and $11.6 \mathrm{~min}$ (major).

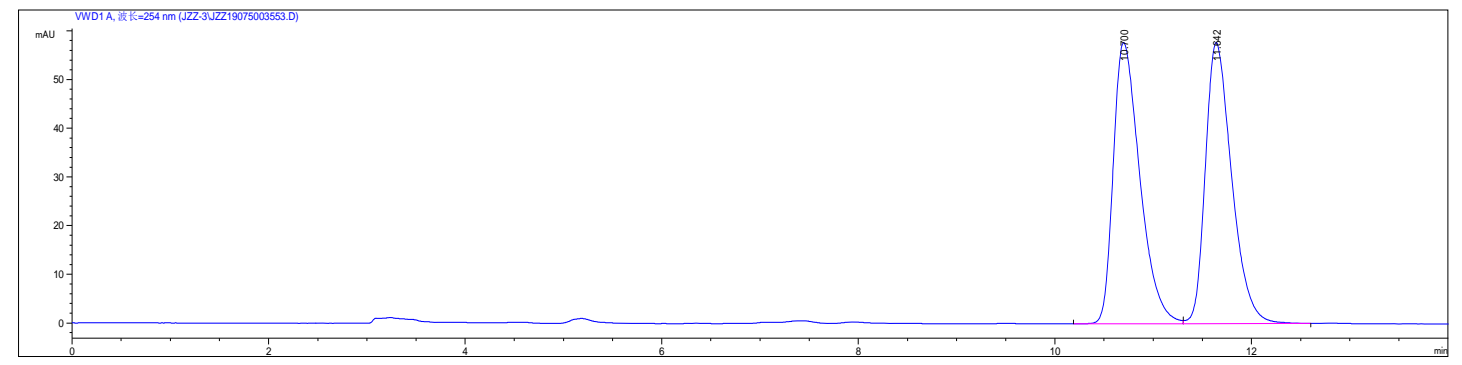




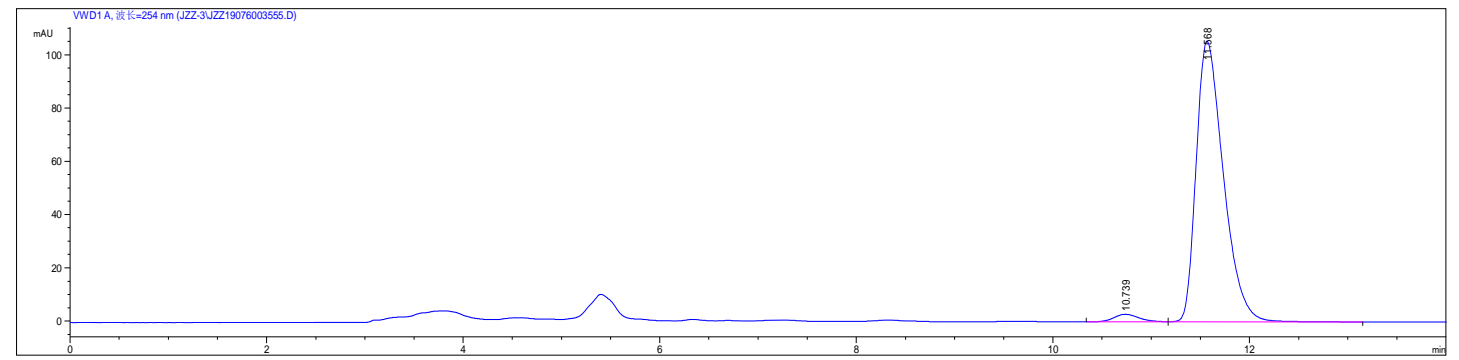

$\mathrm{Ph} \quad \mathrm{Ph} \quad$ Colorless oil; $118.4 \mathrm{mg}(0.04 \mathrm{mmol}), 96 \%$ yield; $>99 \%$ ee; $[\alpha]_{\mathrm{D}}^{26}-16.8$ $\left.\mathrm{CF}_{3}(c) 3.46, \mathrm{CHCl}_{3}\right) ;{ }^{1} \mathrm{H}$ NMR $\left(300 \mathrm{MHz}, \mathrm{CDCl}_{3}\right) \delta 7.53(\mathrm{~d}, J=7.1 \mathrm{~Hz}, 2 \mathrm{H})$, $87.44-7.34(\mathrm{~m}, 3 \mathrm{H}), 7.28-7.24(\mathrm{~m}, 2), 7.19-7.10(\mathrm{~m}, 3 \mathrm{H}), 2.62-2.47(\mathrm{~m}$, 2H), $2.33(\mathrm{~s}, 1 \mathrm{H}), 2.33-2.19(\mathrm{~m}, 1 \mathrm{H}), 2.06-1.96(\mathrm{~m}, 1 \mathrm{H}), 1.67-1.54(\mathrm{~m}, 1 \mathrm{H}), 1.48-1.34(\mathrm{~m}$, 1H), $1.16-1.01(\mathrm{~m}, 1 \mathrm{H}) ;{ }^{13} \mathrm{C} \mathrm{NMR}\left(75 \mathrm{MHz}, \mathrm{CDCl}_{3}\right) \delta 142.1,136.3,128.3$ (three peaks), 126.2 (two peaks), 125.8, $125.6(\mathrm{q}, J=284.0 \mathrm{~Hz}), 77.9,77.4(\mathrm{q}, J=27.7 \mathrm{~Hz}), 35.5,35.0,31.3$, 21.8; HRMS (ESI) m/z 309.1470 (M+H ${ }^{+}$, calc. for $\mathrm{C}_{18} \mathrm{H}_{20} \mathrm{~F}_{3} \mathrm{O} 309.1466$.

The ee was determined by HPLC analysis. CHIRALPAK IB (4.6 mm i.d. x $250 \mathrm{~mm}$ ); Hexane/2-propanol $=95 / 05$; flow rate $1.0 \mathrm{~mL} / \mathrm{min} ; 25{ }^{\circ} \mathrm{C} ; 254 \mathrm{~nm}$; retention time: $7.1 \mathrm{~min}$ (major).
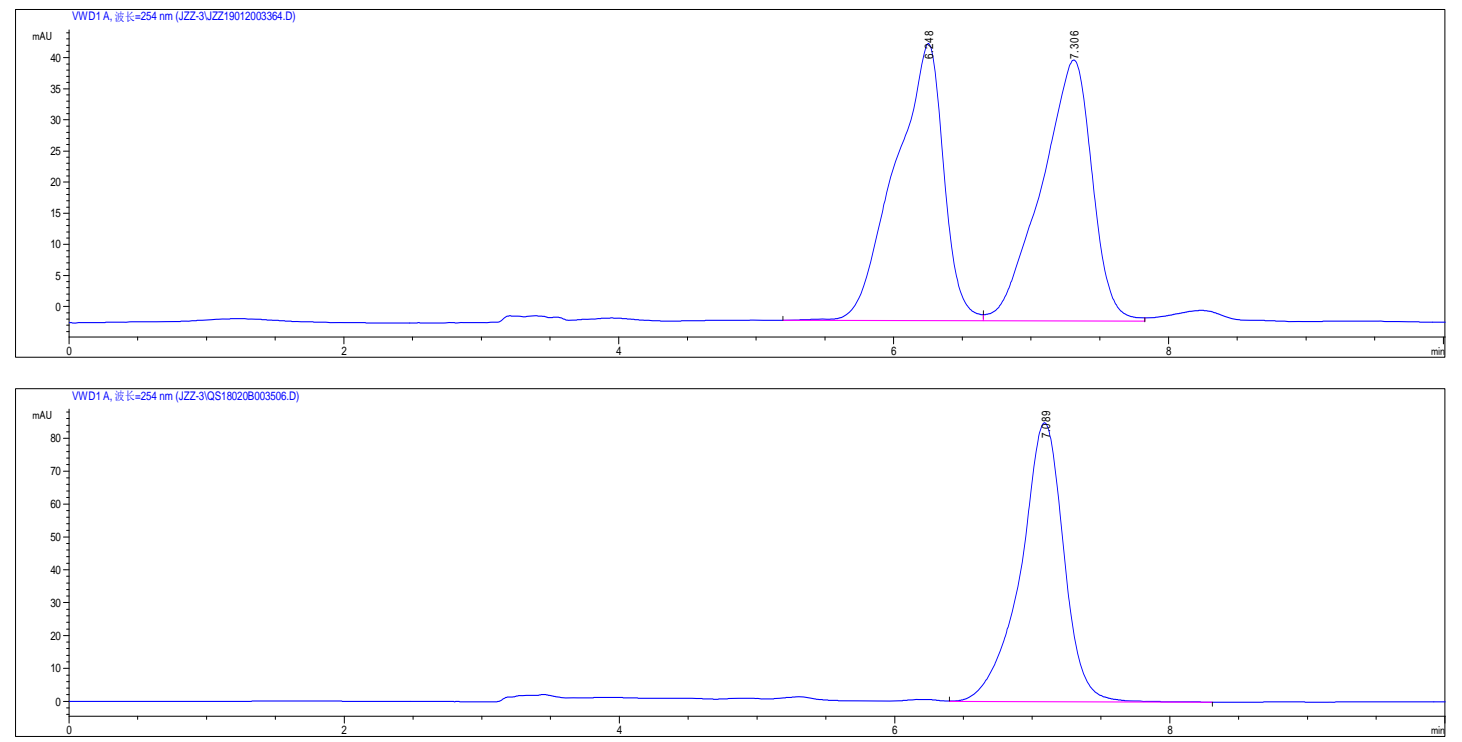

White solid, Mp: 84.2-85.5 ${ }^{\circ} \mathrm{C} ;>99 \%$ ee; $[\alpha]_{\mathrm{D}}^{26}-11.2$ (c 1.17, $\left.\mathrm{CHCl}_{3}\right)$;

$\mathrm{CF}_{3}{ }^{1} \mathrm{H}$ NMR $\left(300 \mathrm{MHz}, \mathrm{CDCl}_{3}\right) \delta 7.57(\mathrm{~d}, J=7.4 \mathrm{~Hz}, 2 \mathrm{H}), 7.43-7.32(\mathrm{~m}$, $3 \mathrm{H}), 5.05(\mathrm{~s}, 1 \mathrm{H}), 3.73-3.55(\mathrm{~m}, 2 \mathrm{H}), 2.52-2.42(\mathrm{~m}, 2 \mathrm{H}), 2.22-2.12(\mathrm{~m}$, 11 1H), 1.56-1.47 (m,2H); ${ }^{13} \mathrm{C}$ NMR (75 MHz, $\left.\mathrm{CDCl}_{3}\right) \delta 137.3,128.2$ (two peaks), 126.7 (two peaks), 125.7 (q, $J=283.8 \mathrm{~Hz}$ ), 76.6 (q, $J=27.6 \mathrm{~Hz}$ ), 62.5, 32.3, 25.0; HRMS (ESI) m/z 235.0949 $\left(\mathrm{M}+\mathrm{H}^{+}\right)$, calc. for $\mathrm{C}_{11} \mathrm{H}_{14} \mathrm{~F}_{3} \mathrm{O}_{2} 235.0946$. 
The ee was determined by HPLC analysis. CHIRALPAK IC and CHIRALPAK IC (4.6 mm i.d. $\mathrm{x} 250 \mathrm{~mm}$ ); Hexane/2-propanol $=90 / 10$; flow rate $1.0 \mathrm{~mL} / \mathrm{min} ; 25^{\circ} \mathrm{C} ; 210 \mathrm{~nm}$; retention time: $9.0 \mathrm{~min}$ (minor), $10.8 \mathrm{~min}$ (major).
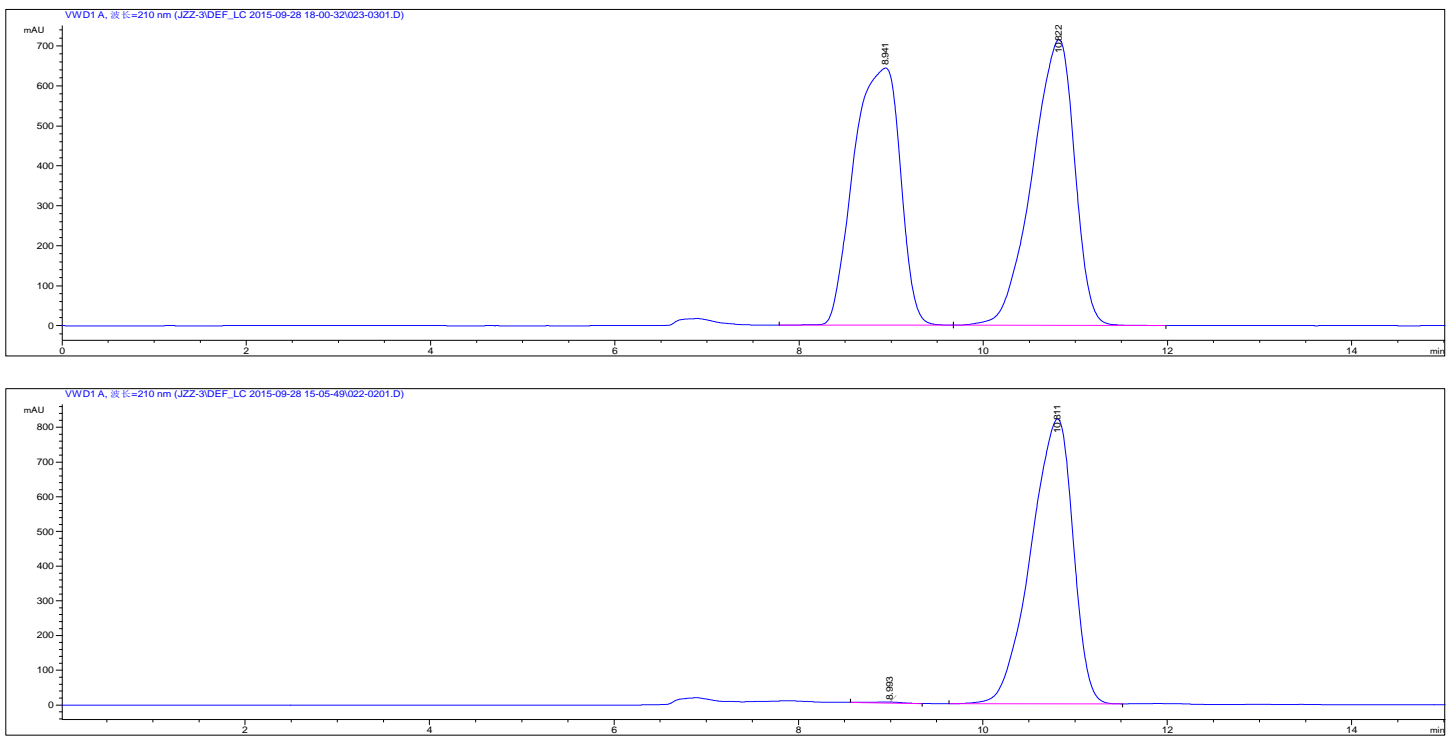

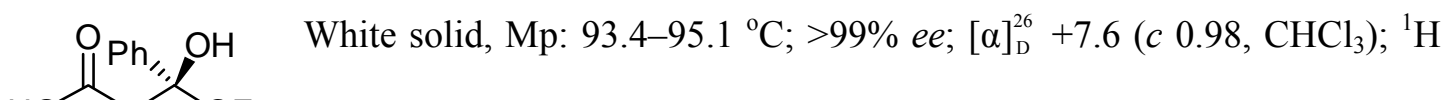
$\mathrm{CF}_{3} \quad \mathrm{NMR}\left(300 \mathrm{MHz}, \mathrm{CDCl}_{3}\right) \delta 7.58-7.55(\mathrm{~m}, 2 \mathrm{H}), 7.44-7.37(\mathrm{~m}, 3 \mathrm{H}), 5.90$ (s, 2H), $3.26(\mathrm{~d}, J=16.6 \mathrm{~Hz}, 1 \mathrm{H}), 3.17(\mathrm{~d}, J=16.6 \mathrm{~Hz}, 1 \mathrm{H}) ;{ }^{13} \mathrm{C} \mathrm{NMR}$ $12\left(75 \mathrm{MHz}, \mathrm{CDCl}_{3}\right) \delta 175.7,136.5,129.1,128.5,126.3,124.4(\mathrm{q}, J=283.1$ $\mathrm{Hz}), 75.2(\mathrm{q}, J=29.4 \mathrm{~Hz}), 38.1$; HRMS (ESI) m/z $235.0589\left(\mathrm{M}+\mathrm{H}^{+}\right)$, calc. for $\mathrm{C}_{10} \mathrm{H}_{10} \mathrm{~F}_{3} \mathrm{O}_{3}$ 235.0582 .

The ee was determined by HPLC analysis. CHIRLCEL OJ (4.6 mm i.d. x $250 \mathrm{~mm}$ ); Hexane/2-propanol/TFA $=50 / 10 / 1$; flow rate $1.0 \mathrm{~mL} / \mathrm{min} ; 25^{\circ} \mathrm{C} ; 254 \mathrm{~nm}$; retention time: 60.2 $\min$ (major).
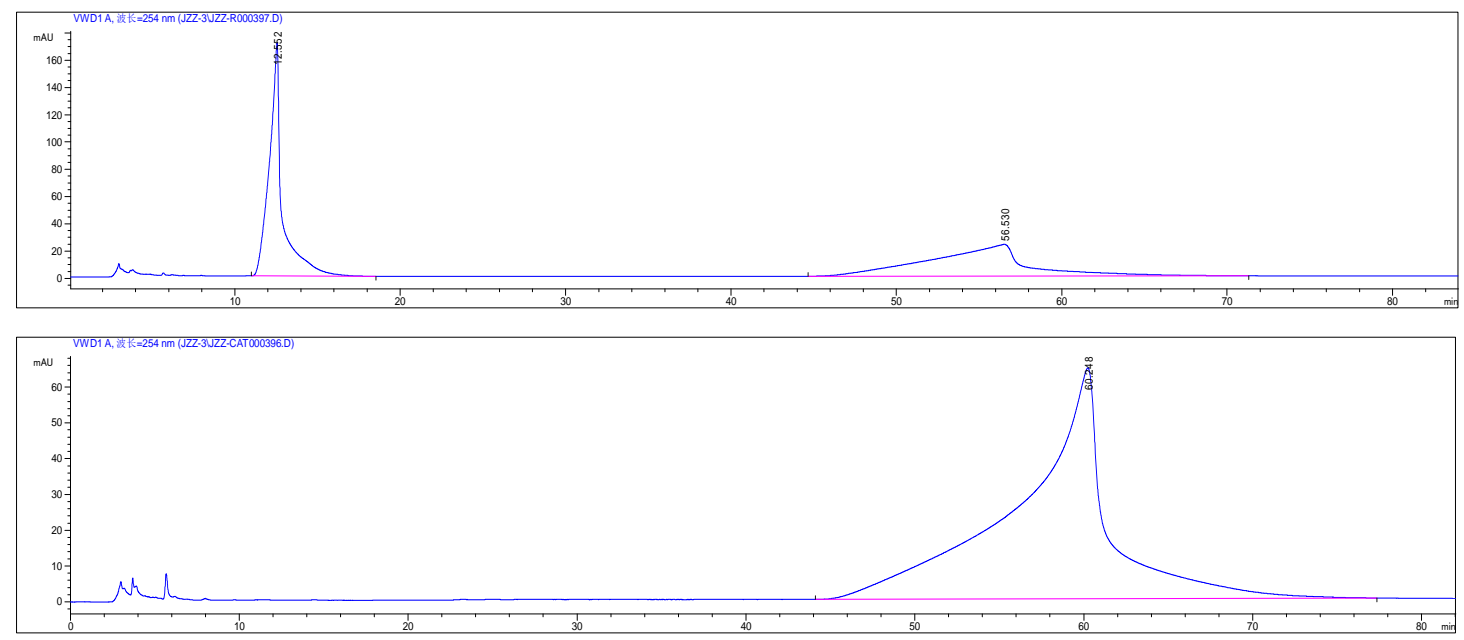
$\begin{array}{ll}\mathrm{OH} & \left.\text { Colorless oil; }>99 \% e e ;[\alpha]_{\mathrm{D}}^{26}+142.9(c) 2.38, \mathrm{CHCl}_{3}\right) ;{ }^{1} \mathrm{H} \text { NMR }(300 \mathrm{MHz} \text {, }\end{array}$ 13 7.40-7.30 (m, 3H), $5.68(\mathrm{~s}, 1 \mathrm{H}), 4.05(\mathrm{~d}, J=17.4 \mathrm{~Hz}, 1 \mathrm{H}), 3.65(\mathrm{~d}, J=$ $17.4 \mathrm{~Hz}, 1 \mathrm{H}) . ;{ }^{13} \mathrm{C}$ NMR $\left(75 \mathrm{MHz}, \mathrm{CDCl}_{3}\right) \delta 199.6,137.6,136.2,134.4,128.9,128.7,128.4$, 128.2, 126.3 (two peaks), 124.5 (q, $J=282.9 \mathrm{~Hz}), 76.5(\mathrm{q}, J=28.8 \mathrm{~Hz}), 40.2$; HRMS (ESI) $\mathrm{m} / \mathrm{z} 295.0945\left(\mathrm{M}+\mathrm{H}^{+}\right)$, calc. for $\mathrm{C}_{16} \mathrm{H}_{14} \mathrm{~F}_{3} \mathrm{O}_{2} 295.0946$.

The ee was determined by HPLC analysis. CHIRALPAK IB (4.6 mm i.d. x $250 \mathrm{~mm})$; Hexane $/ 2$-propanol $=90 / 10$; flow rate $1.0 \mathrm{~mL} / \mathrm{min} ; 25{ }^{\circ} \mathrm{C} ; 254 \mathrm{~nm}$; retention time: $5.9 \mathrm{~min}$ (minor), $7.4 \min$ (major).
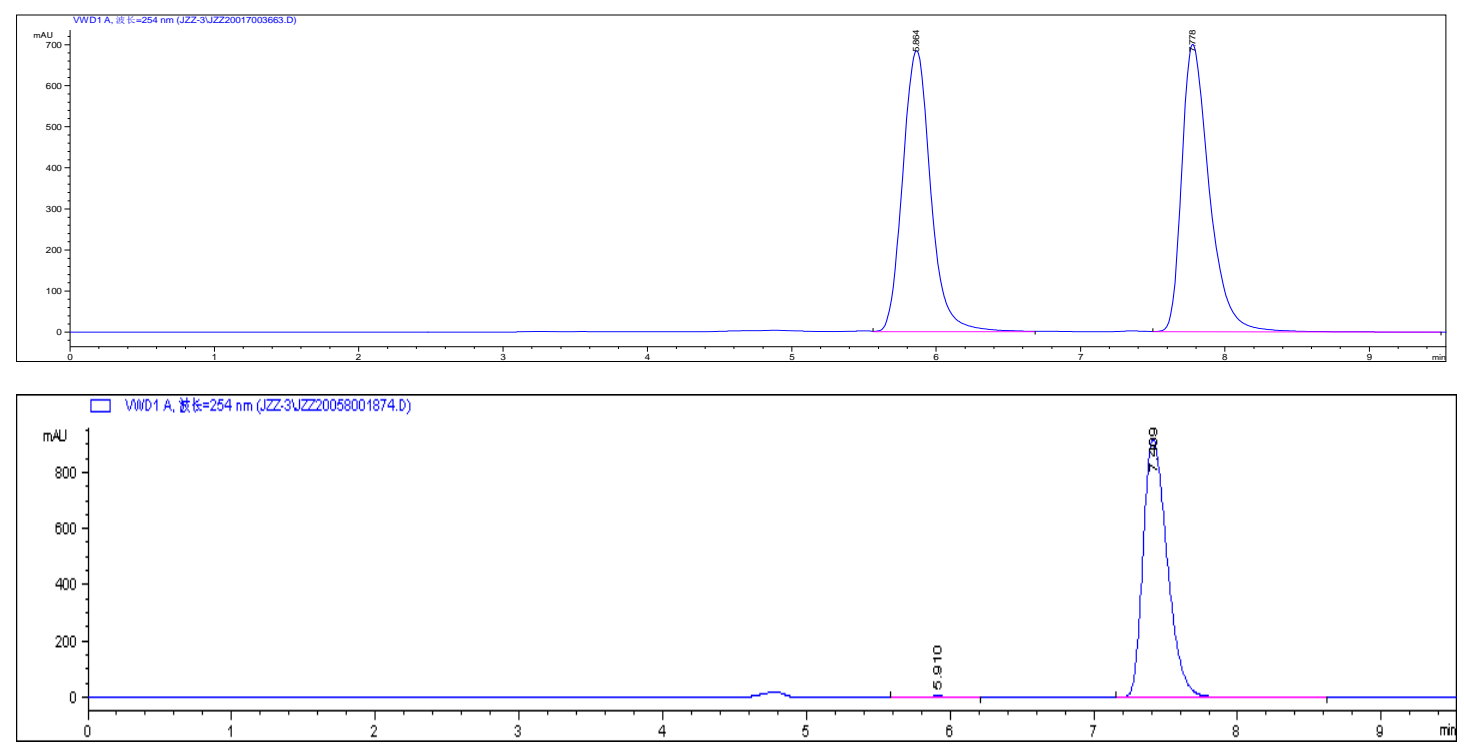<smiles>CO[C@@](C(=O)O)(c1ccccc1)C(F)(F)F</smiles>

(R)-Mosher'sacid

Colorless oil; $(R)$-Mosher's acid was assigned by comparison of their optical rotation $\left\{[\alpha]_{\mathrm{D}}^{26}+44.4\right.$ (c $\left.\left.1.50, \mathrm{CHCl}_{3}\right)\right\}$ with that of commercial $(R)$-Mosher's acid $\left\{[\alpha]_{\mathrm{D}}^{26}+44.0\left(c 1.50, \mathrm{CHCl}_{3}\right)\right\} ;{ }^{1} \mathrm{H} \mathrm{NMR}(300 \mathrm{MHz}$, $\left.\mathrm{CDCl}_{3}\right) \delta 7.83(\mathrm{~s}, 1 \mathrm{H}), 7.60-7.57(\mathrm{~m}, 2 \mathrm{H}), 7.45-7.43(\mathrm{~m}, 3 \mathrm{H}), 3.57(\mathrm{~s}$, $1 \mathrm{H}) ;{ }^{13} \mathrm{C}$ NMR $\left(75 \mathrm{MHz}, \mathrm{CDCl}_{3}\right) \delta 171.0,131.0,130.0,128.7,127.4$ (two peaks), 123.0 (q, $J$ $=286.9 \mathrm{~Hz}), 84.3(\mathrm{q}, J=27.9 \mathrm{~Hz}), 55.6$; HRMS (ESI) $\mathrm{m} / \mathrm{z} 235.0576\left(\mathrm{M}+\mathrm{H}^{+}\right)$, calc. for $\mathrm{C}_{10} \mathrm{H}_{10} \mathrm{~F}_{3} \mathrm{O}_{3} 235.0582$. 
5. Determination of the absolute configuration by X-ray crystallograph

Absolute configurations of $\mathbf{3}, \mathbf{6}$ and all derivatives are determined by X-ray structure analysis of the product $3 \mathbf{e}$.

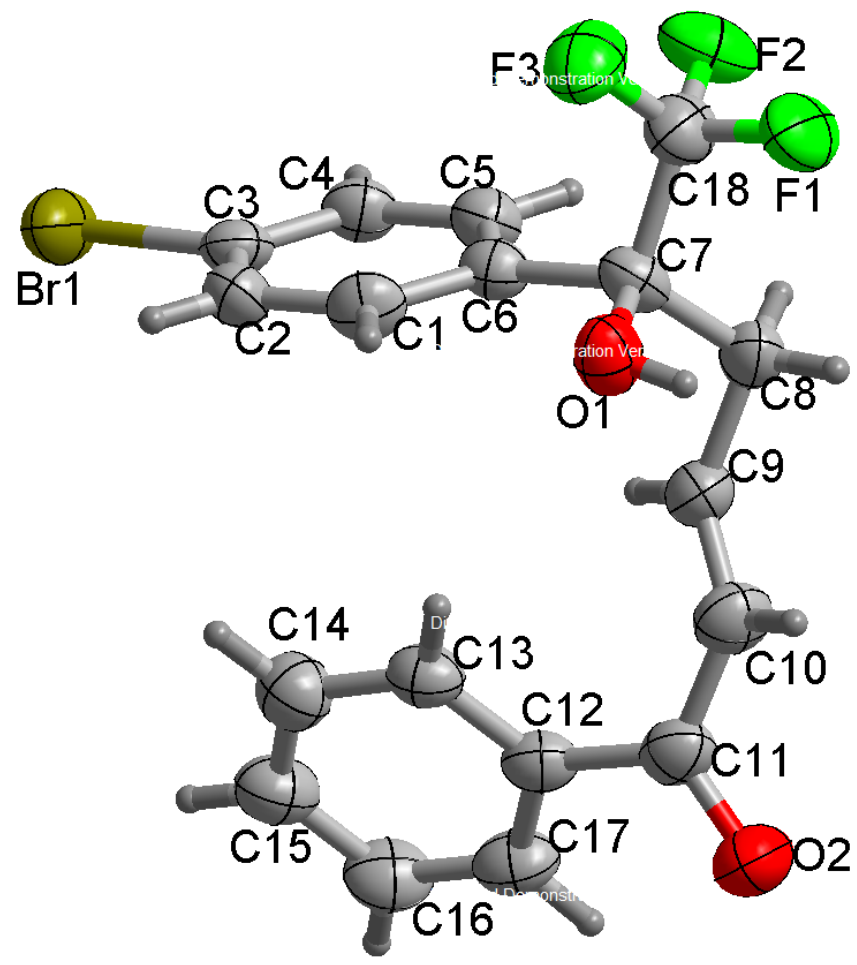

CCDC 1438407 
6. Plausible transition state

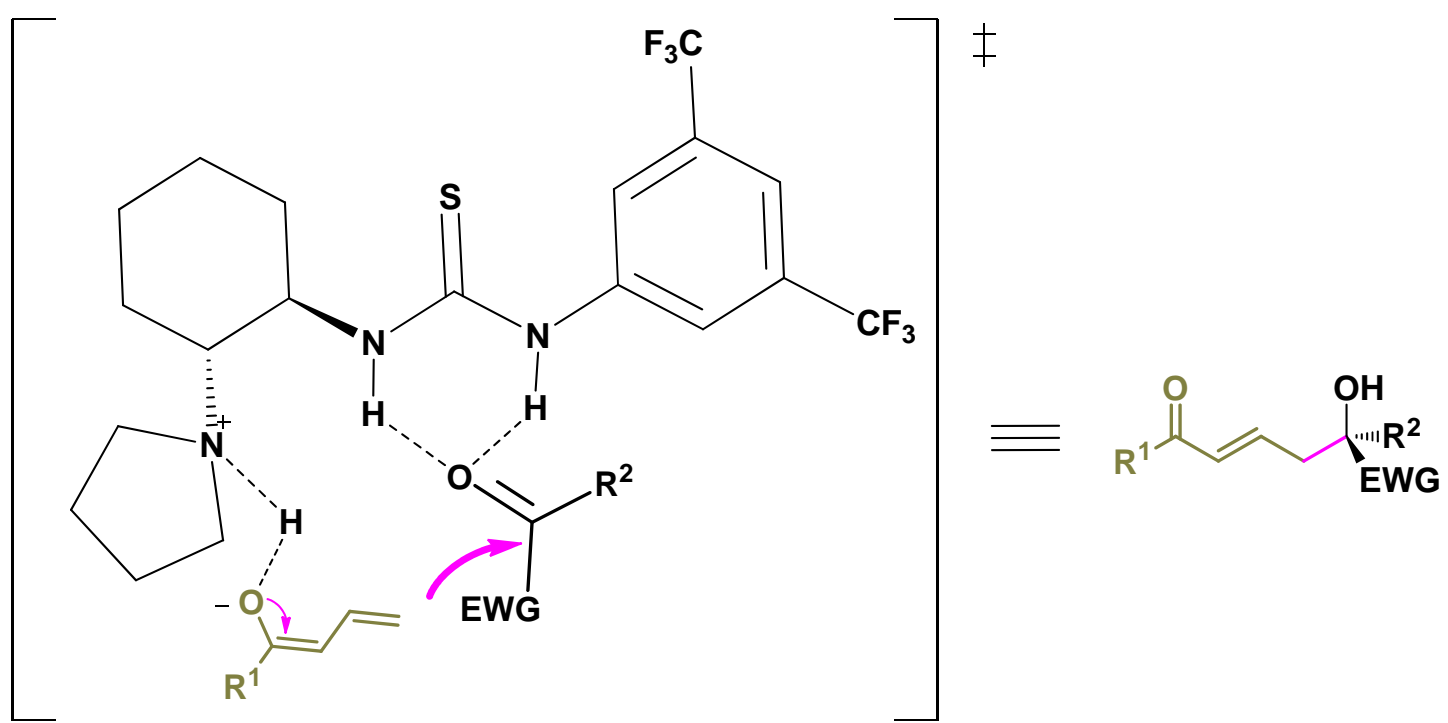




\section{Copies of NMR spectra}

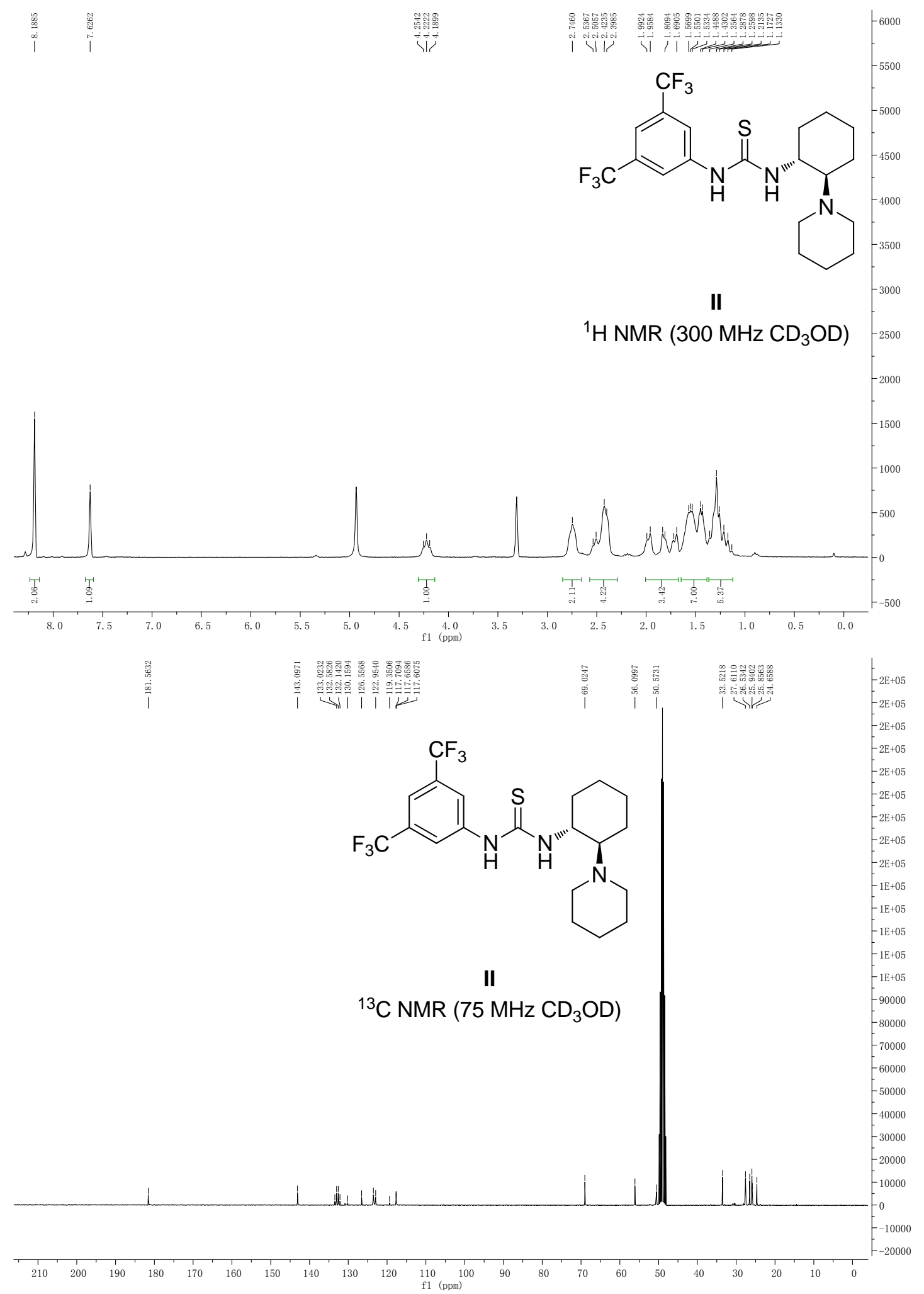




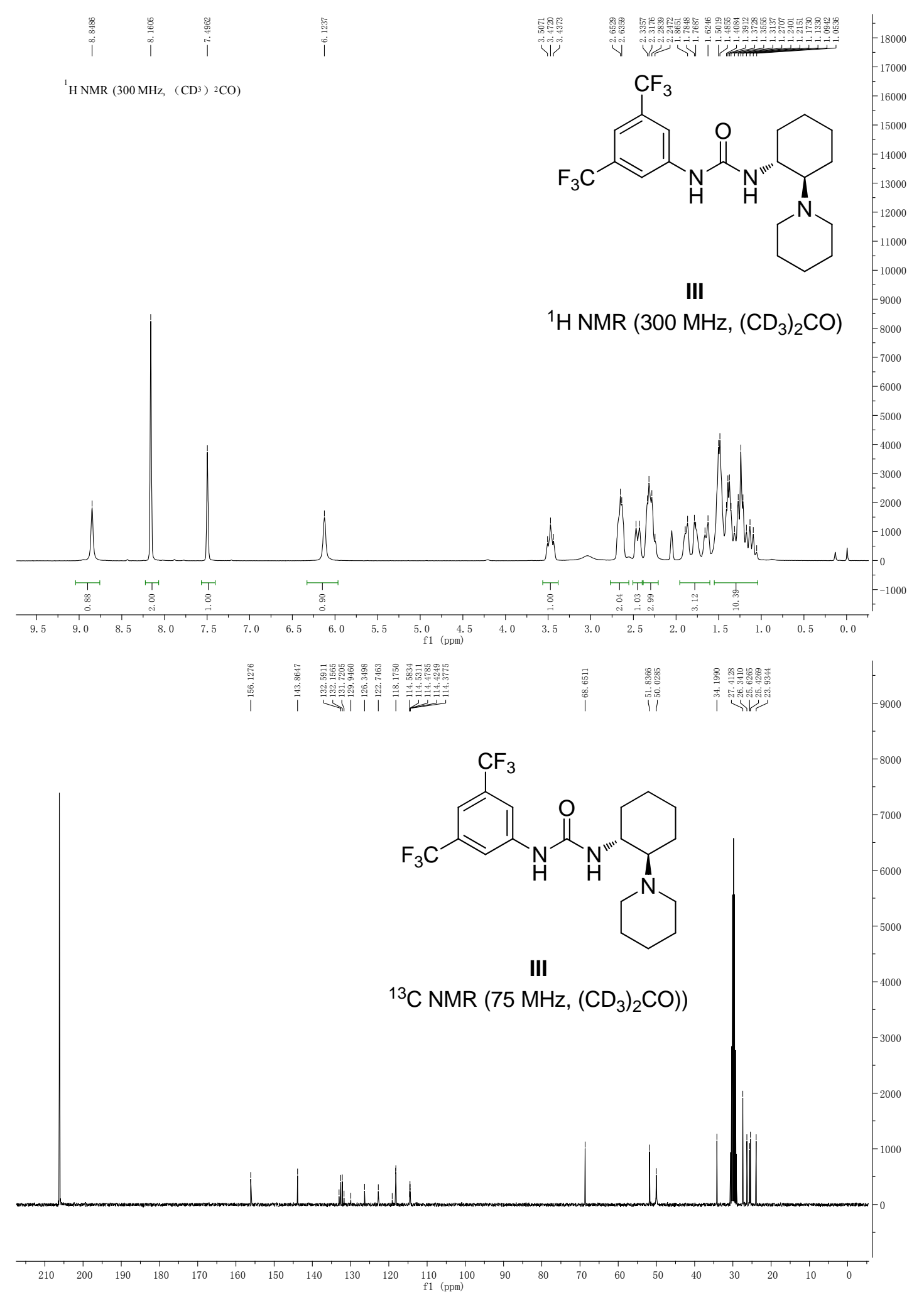




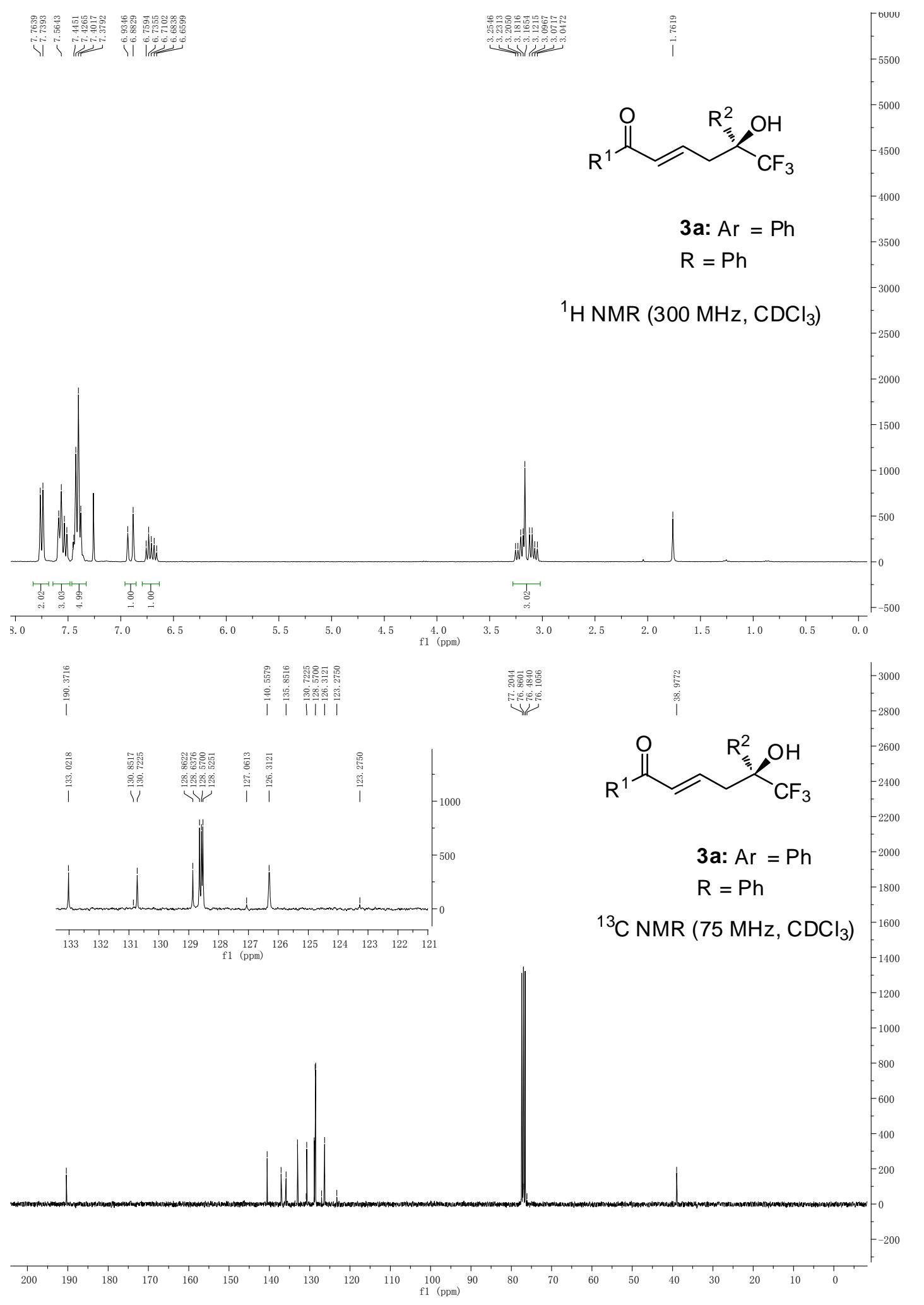




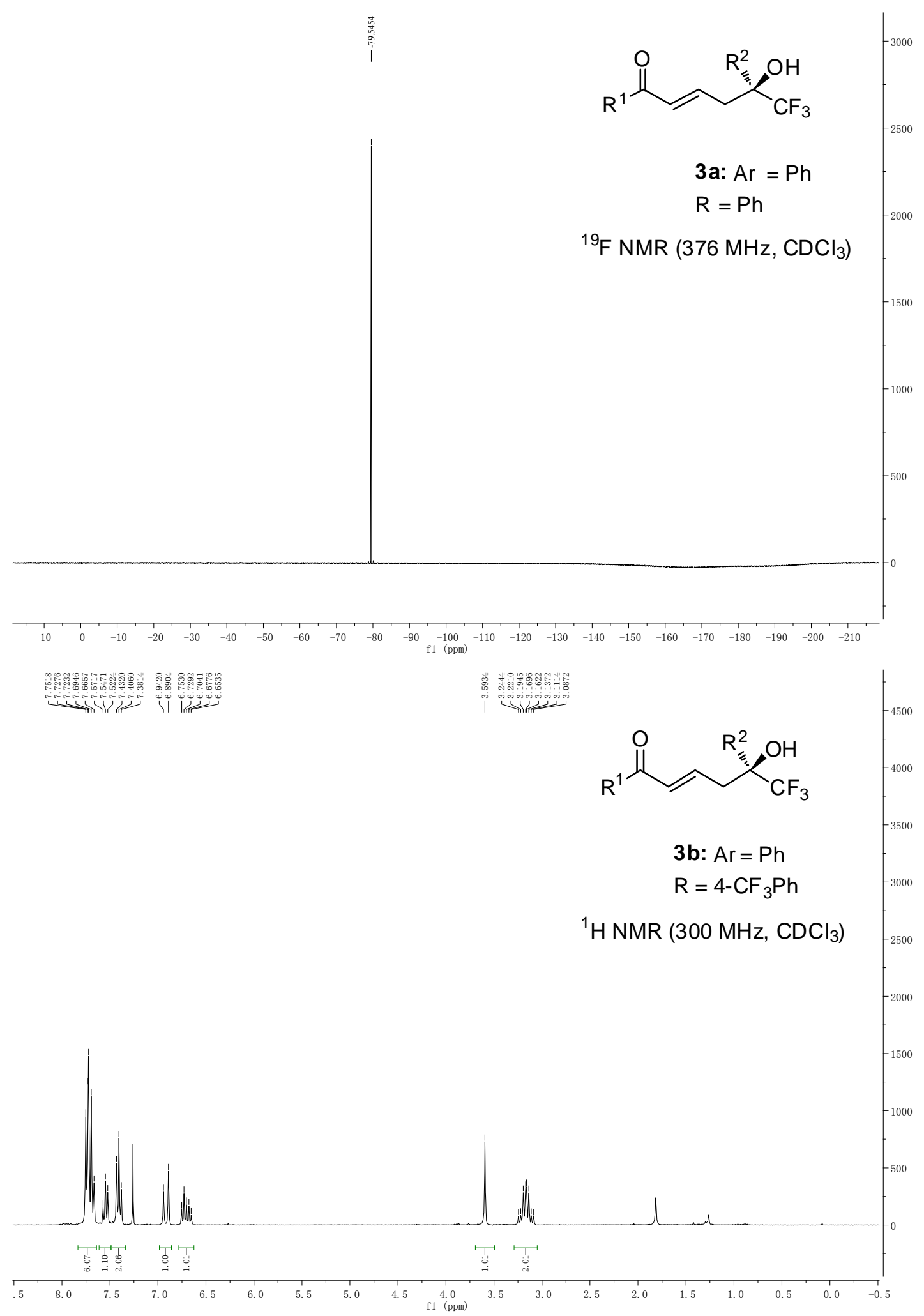




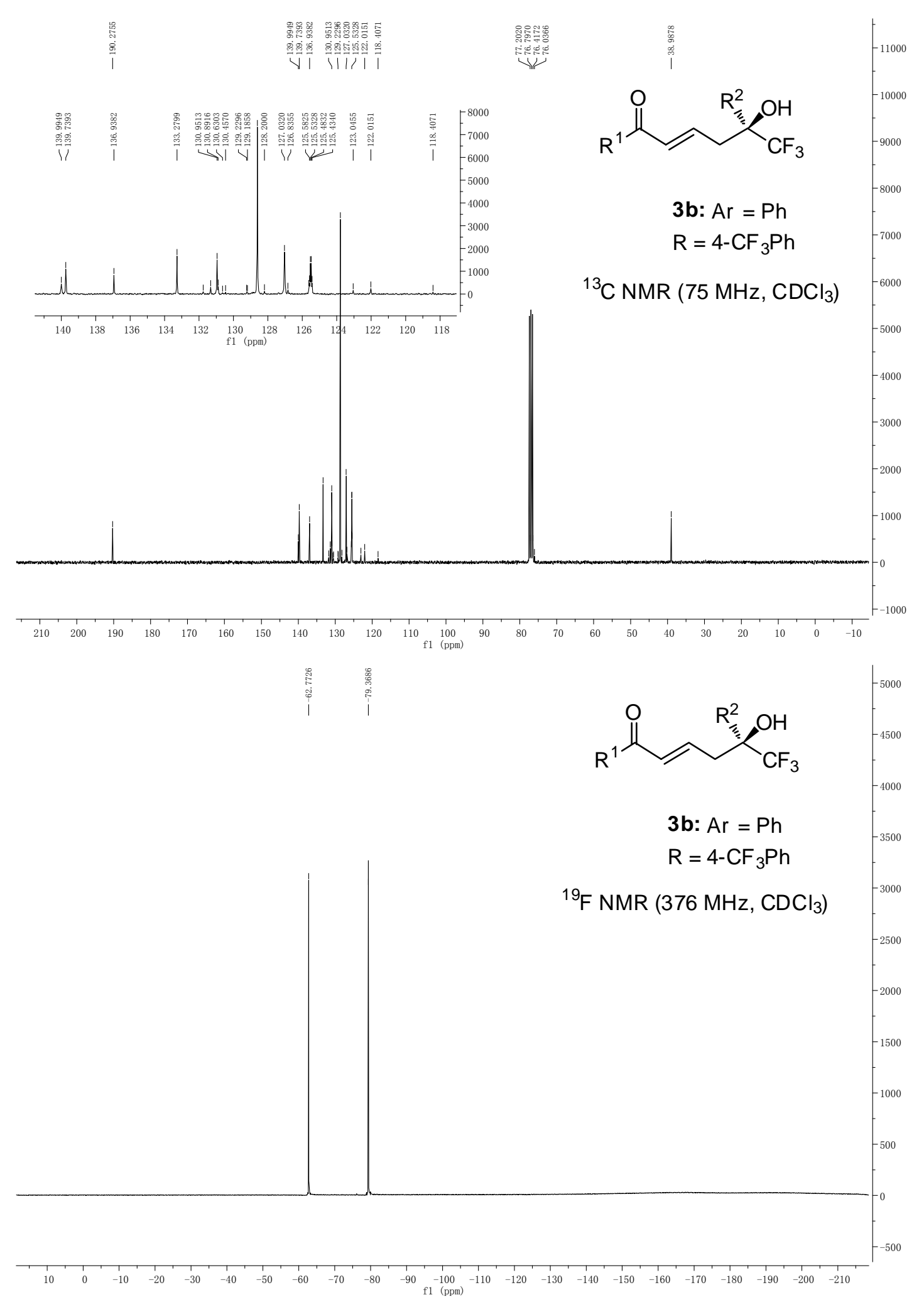




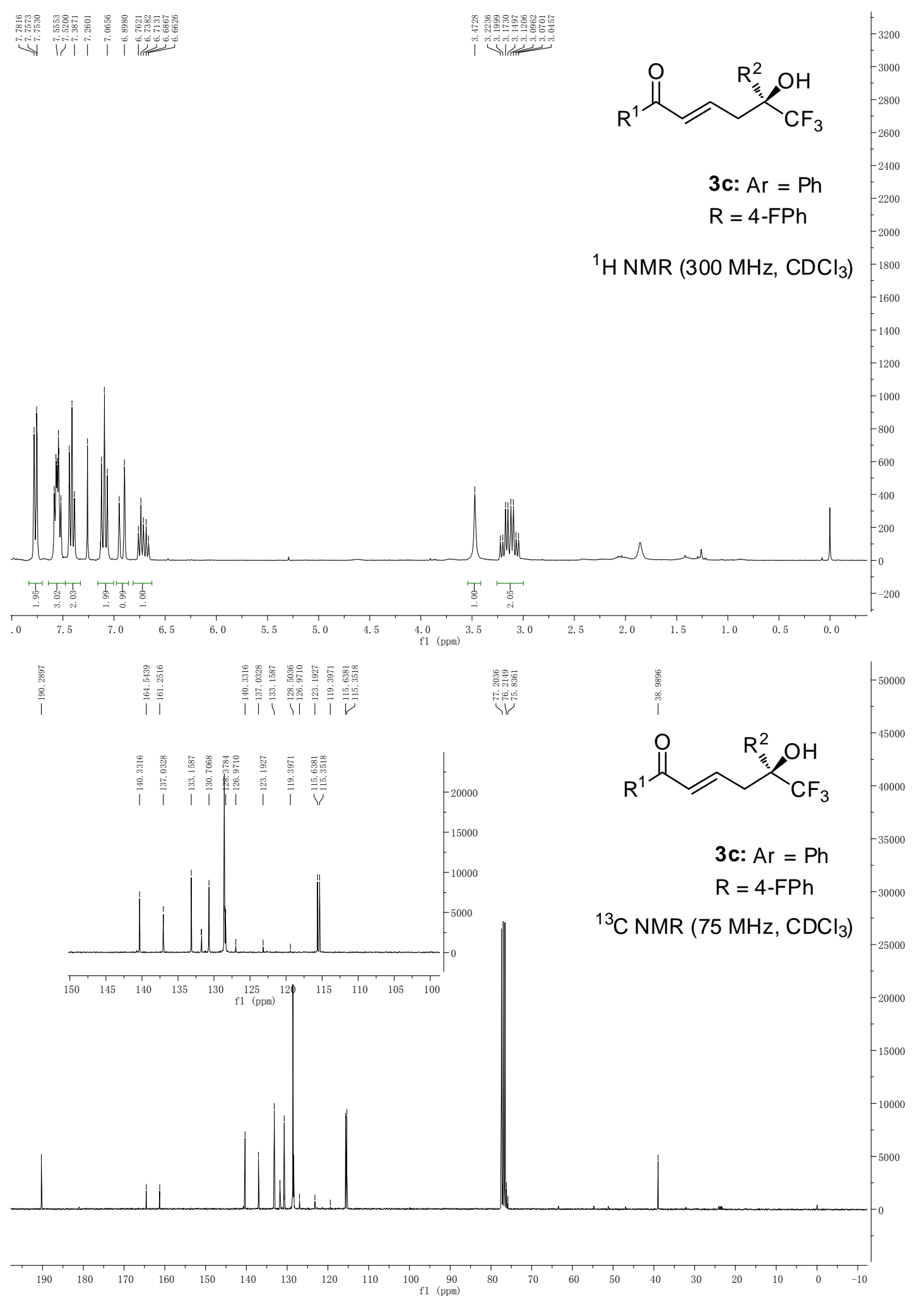




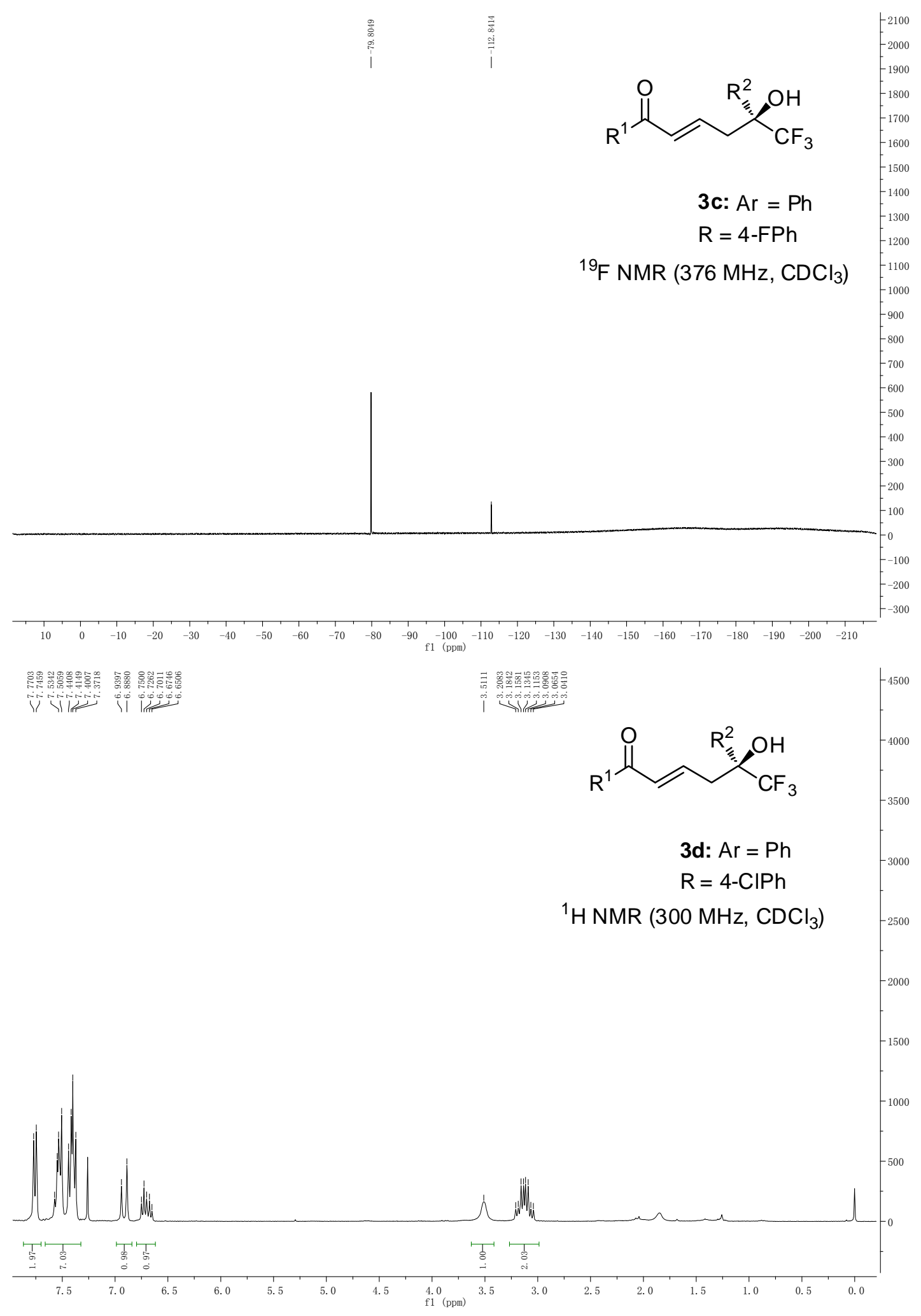




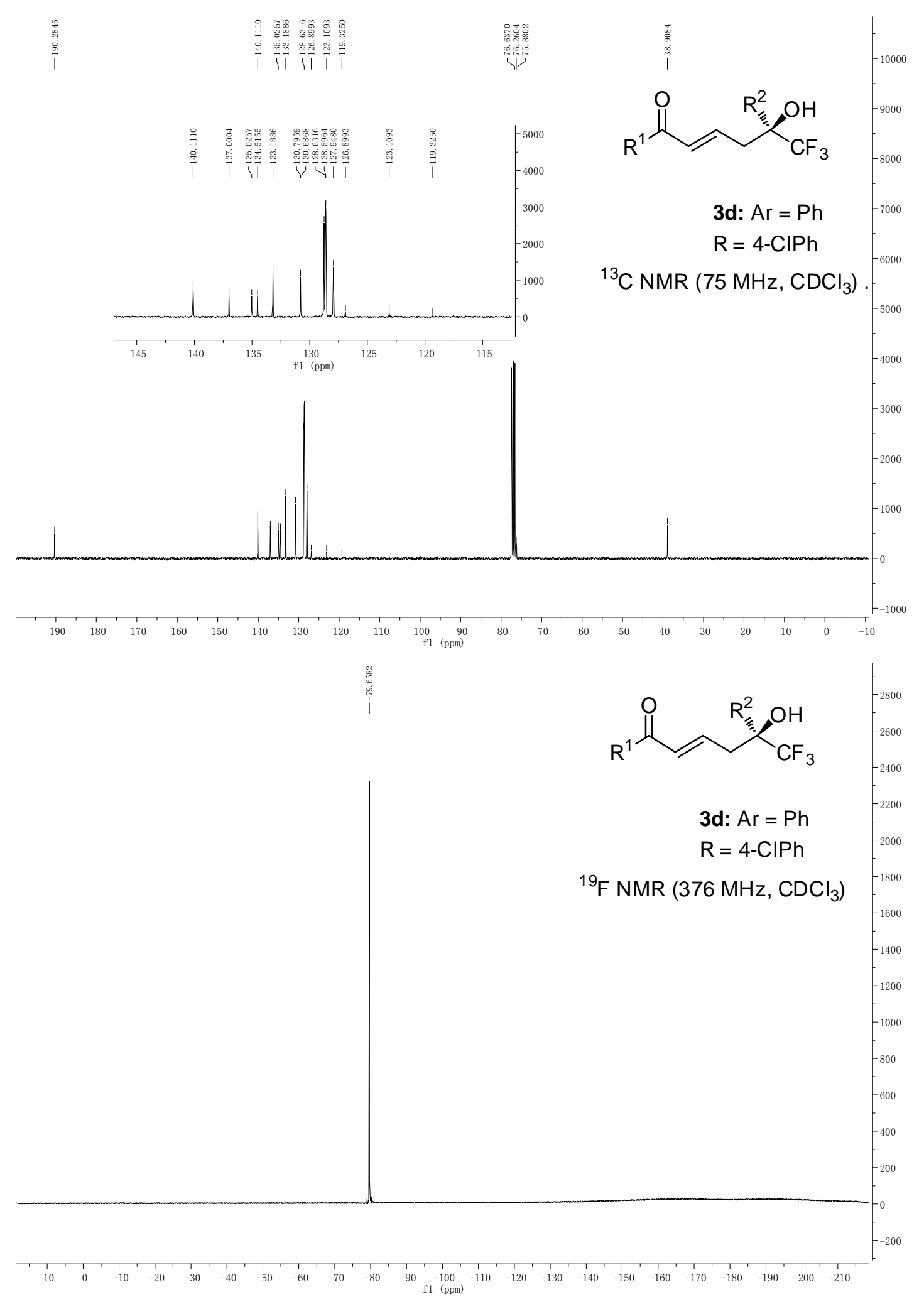




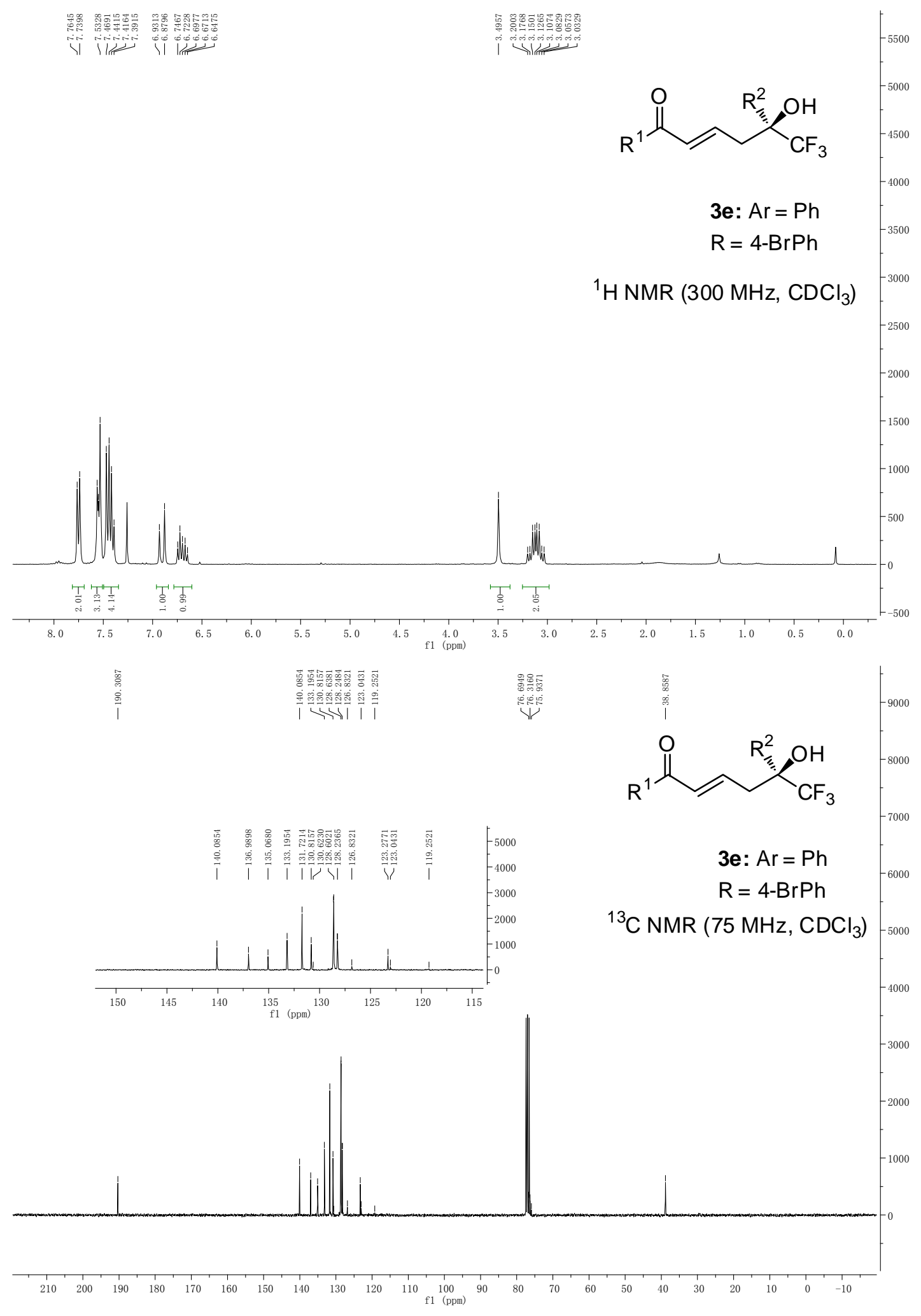




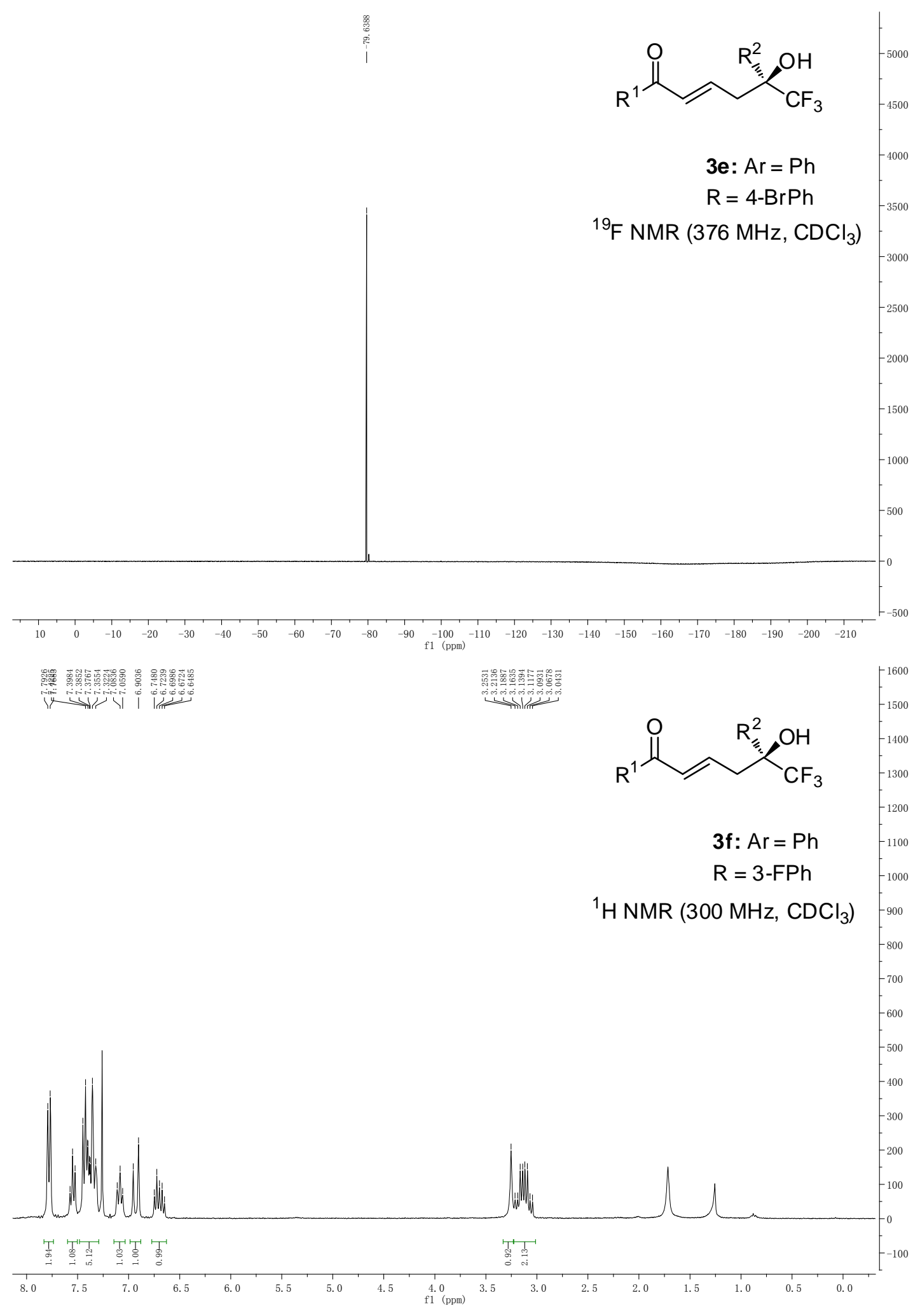




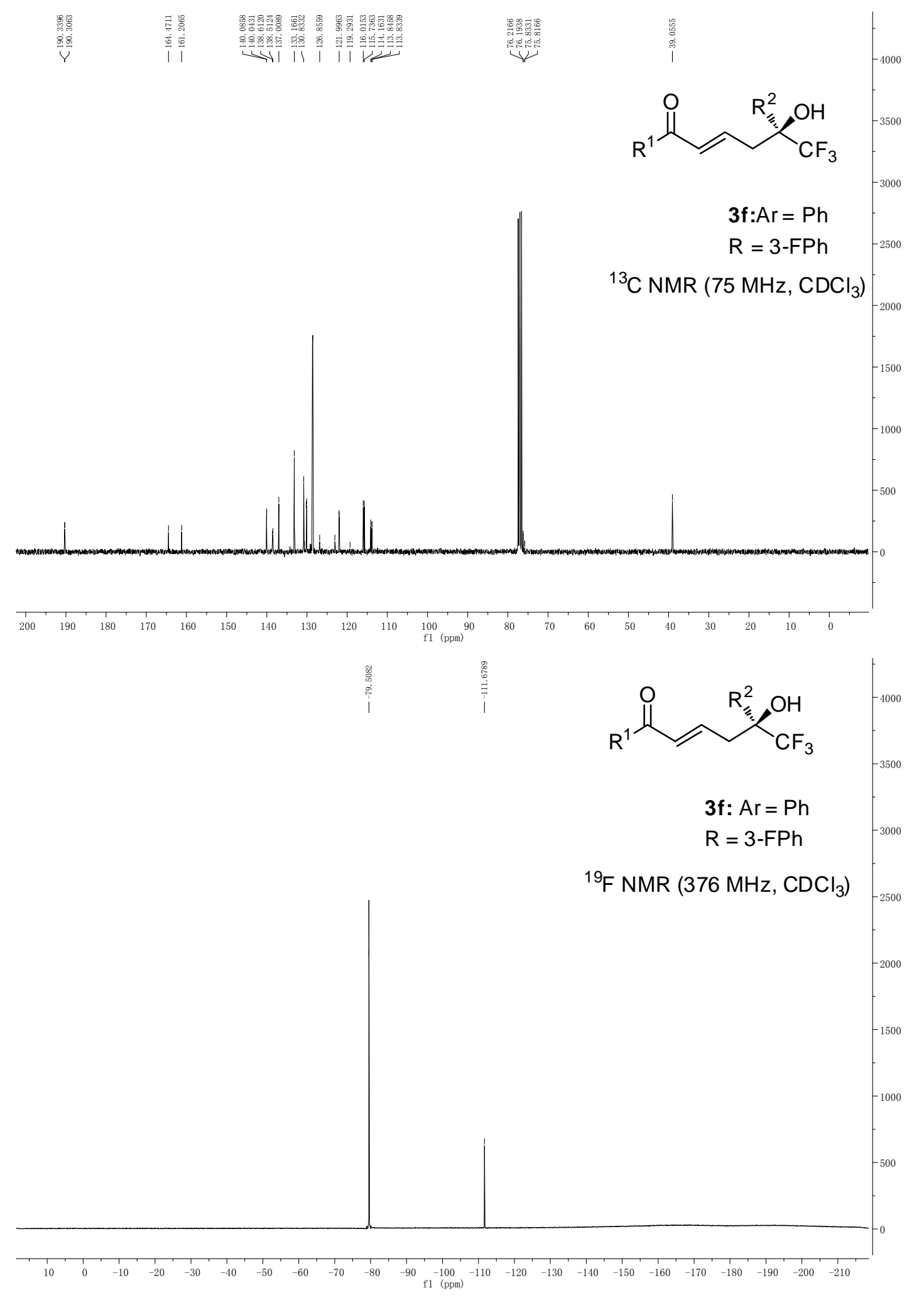




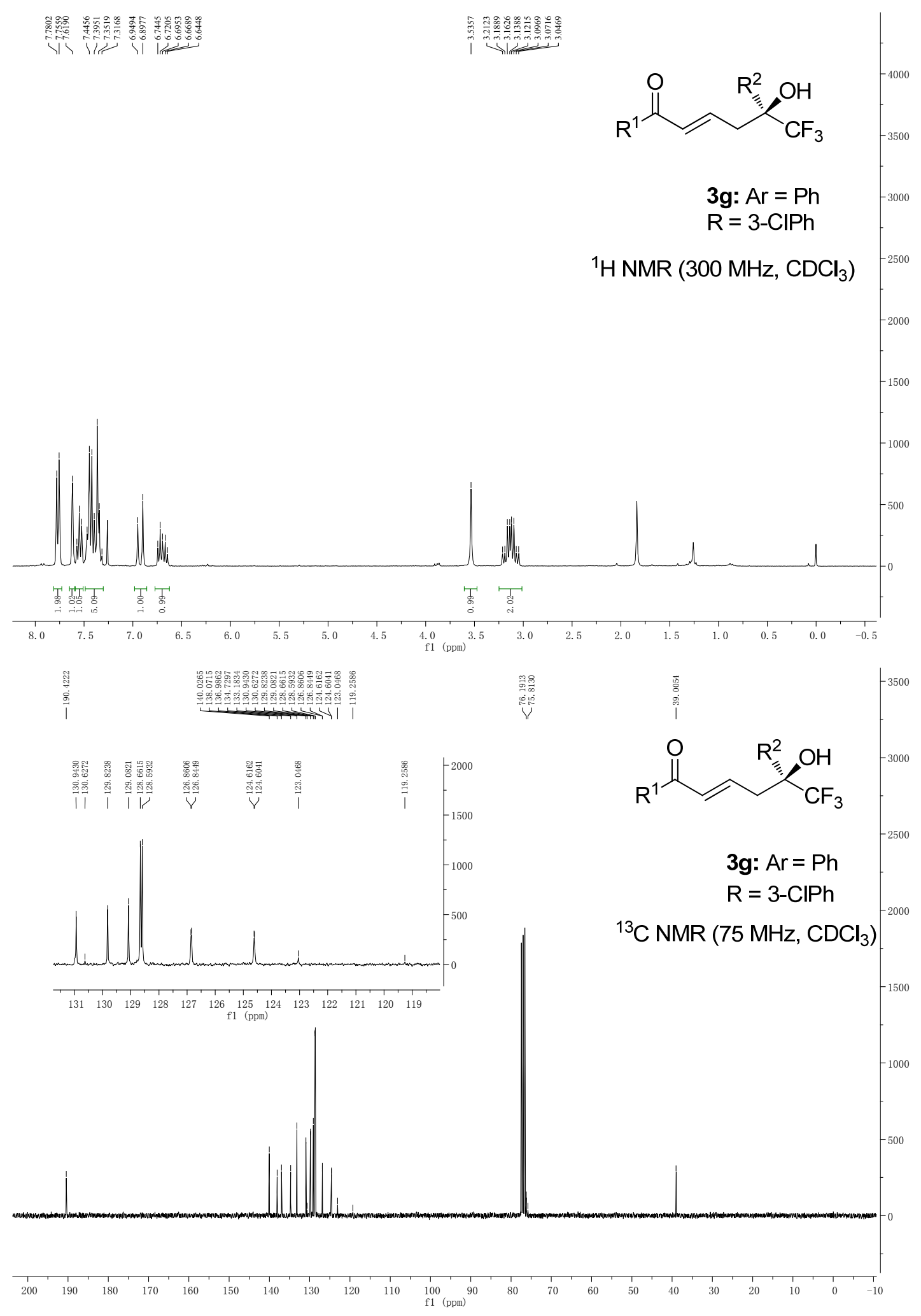




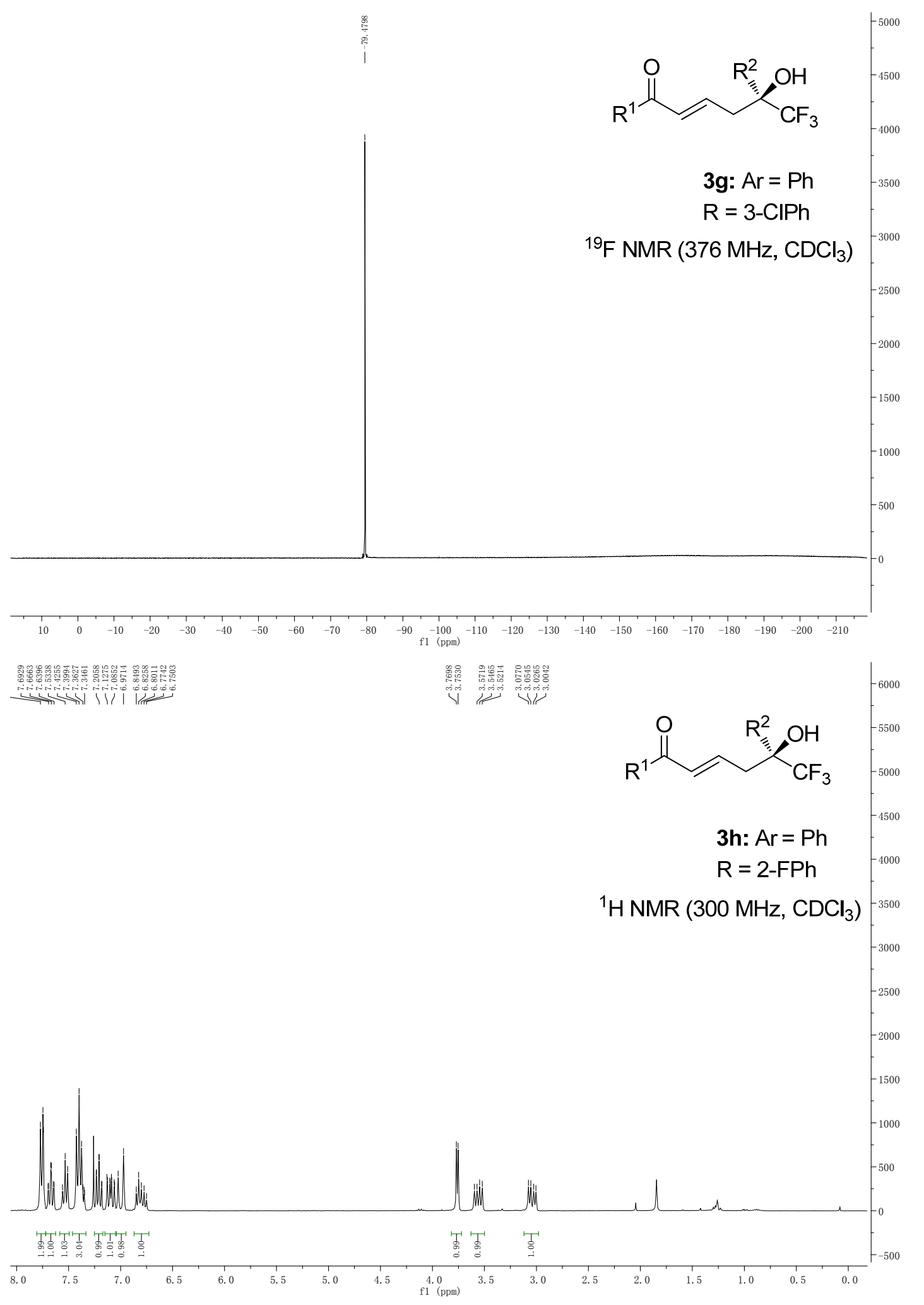




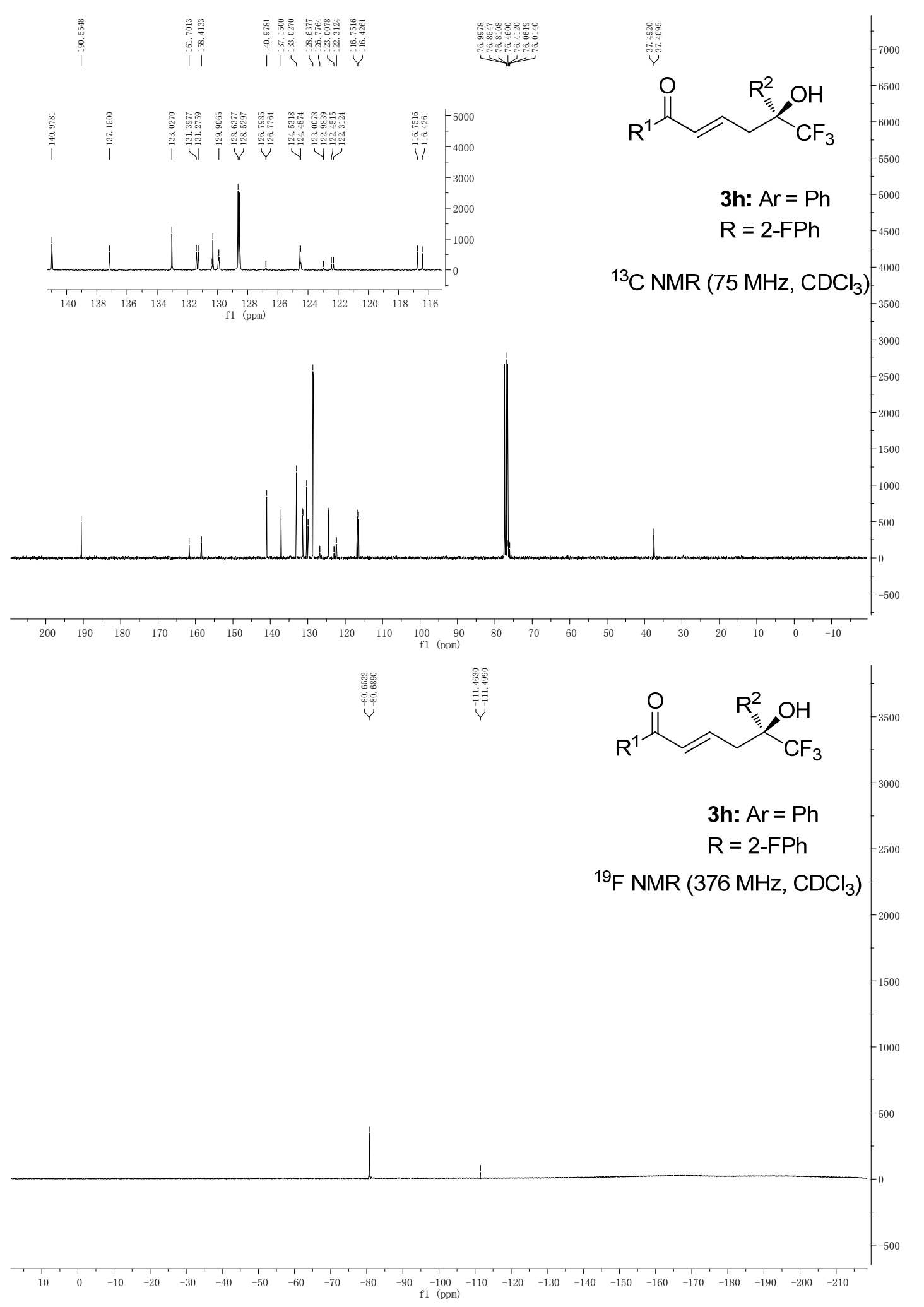




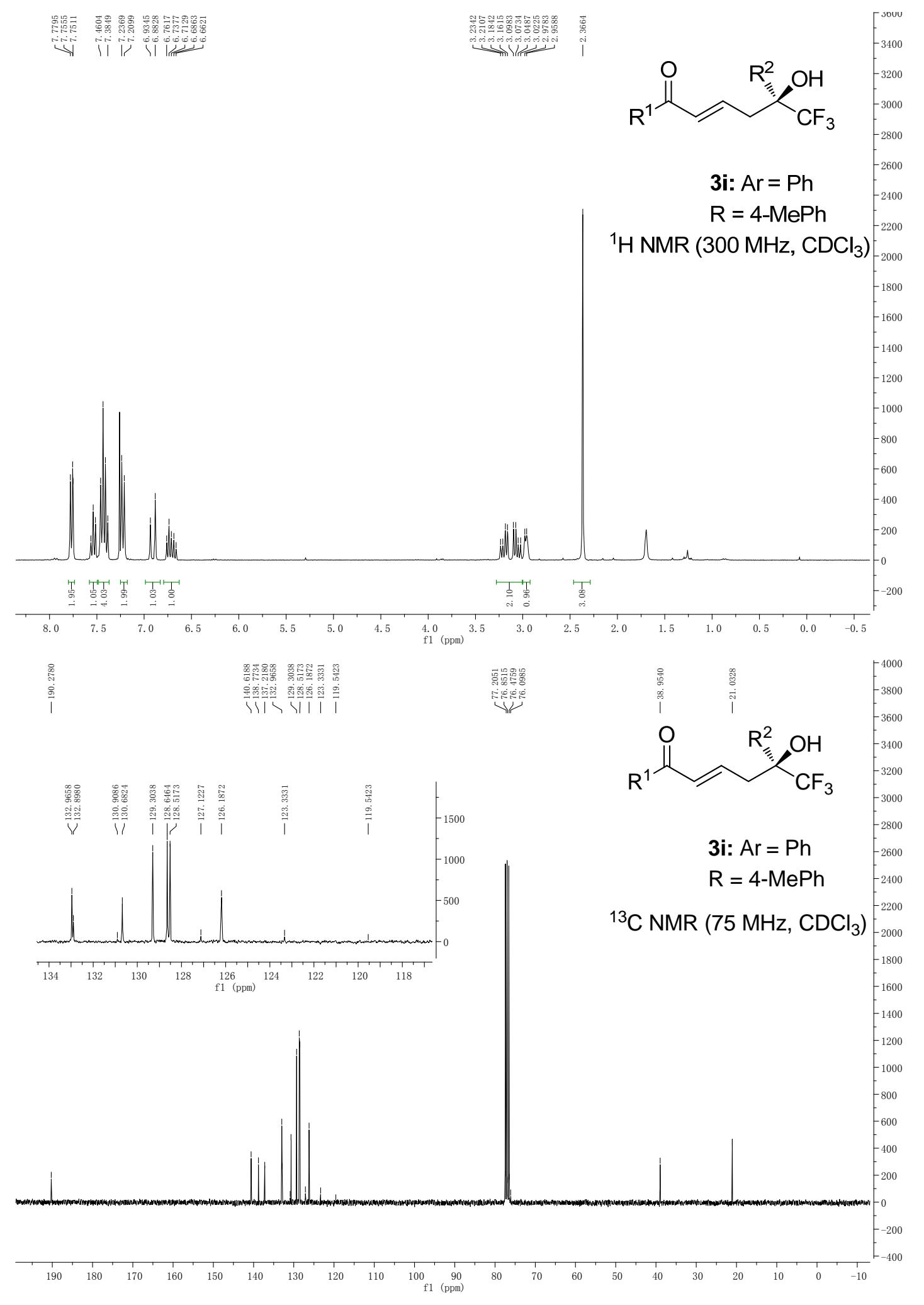




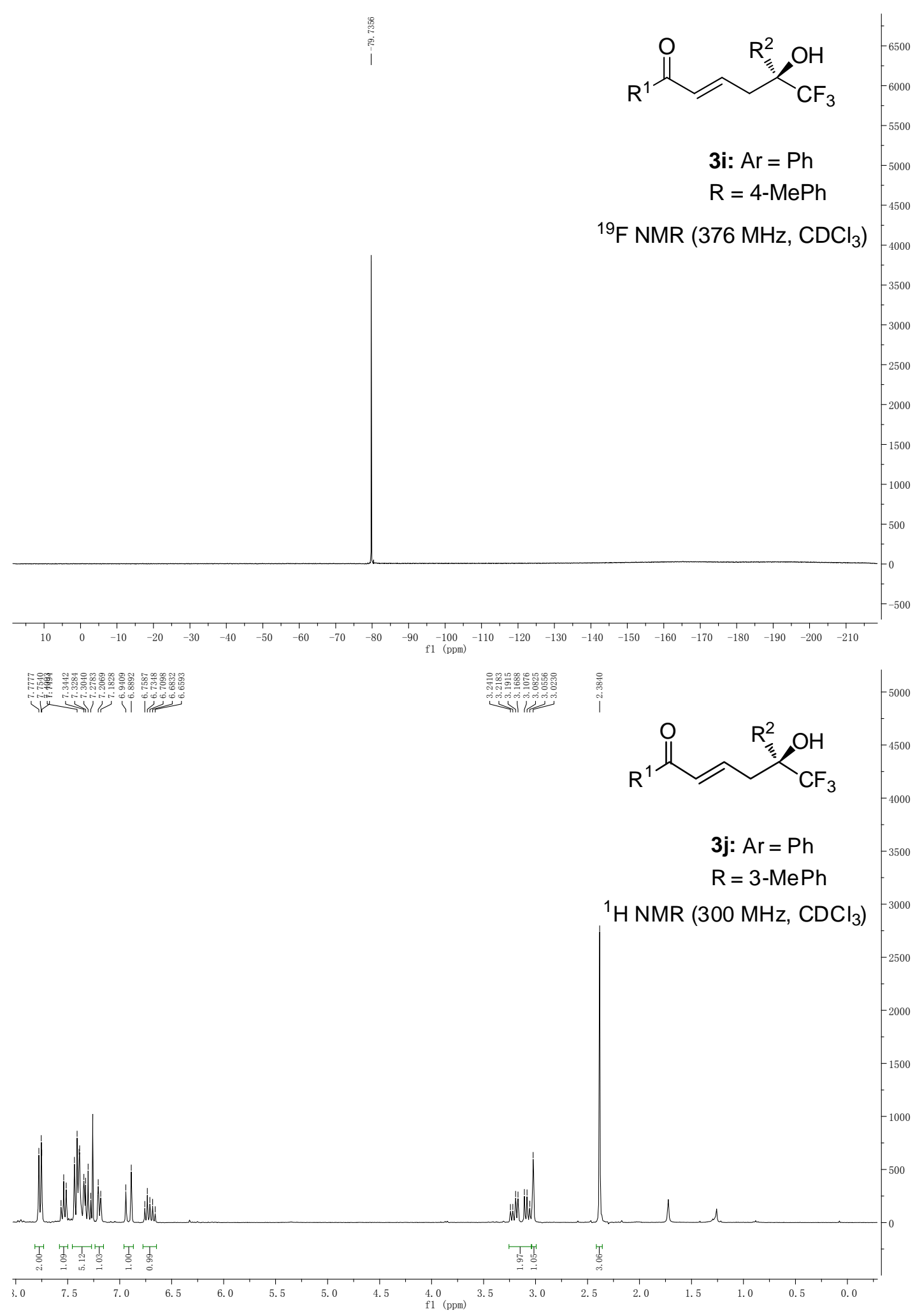




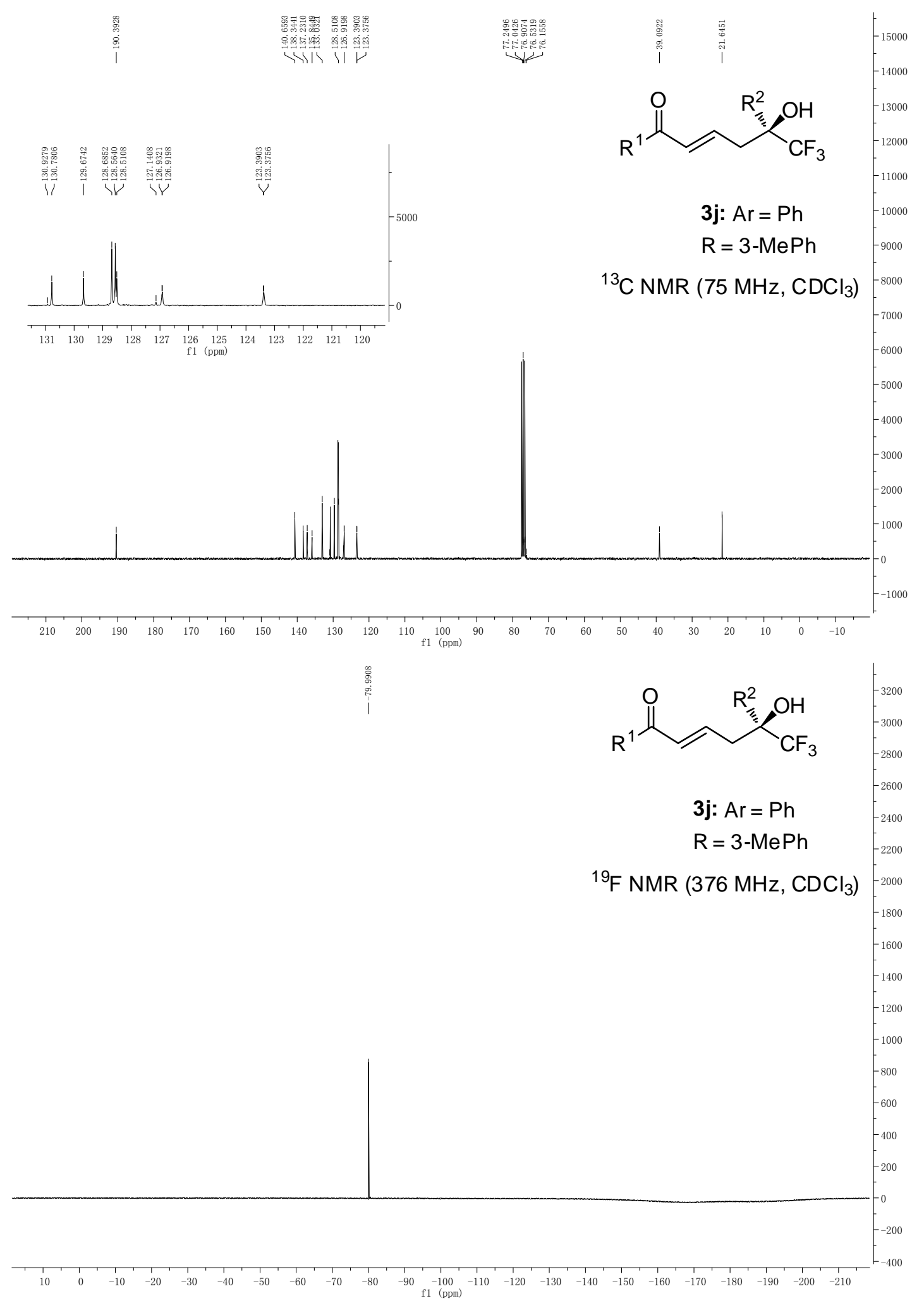




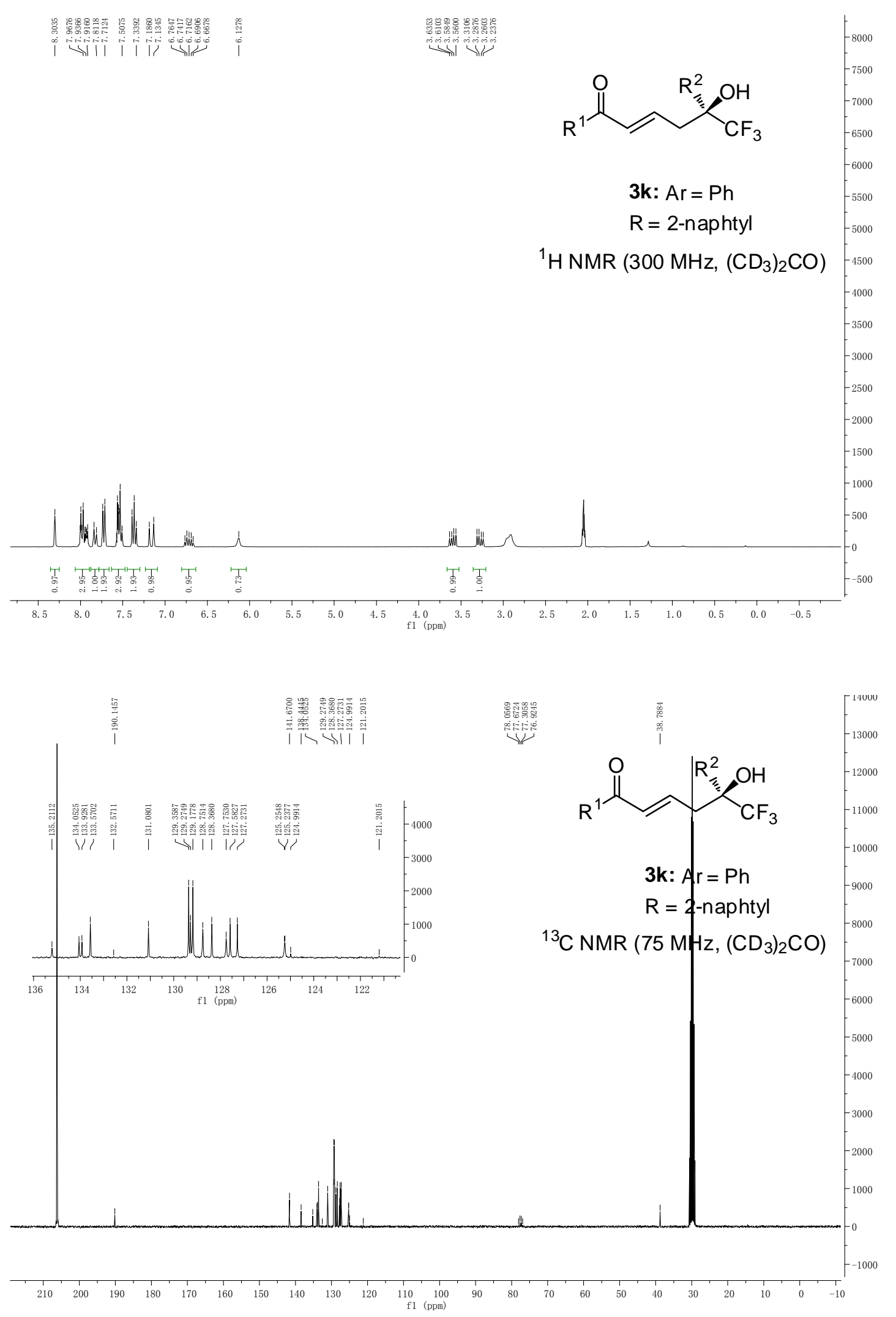




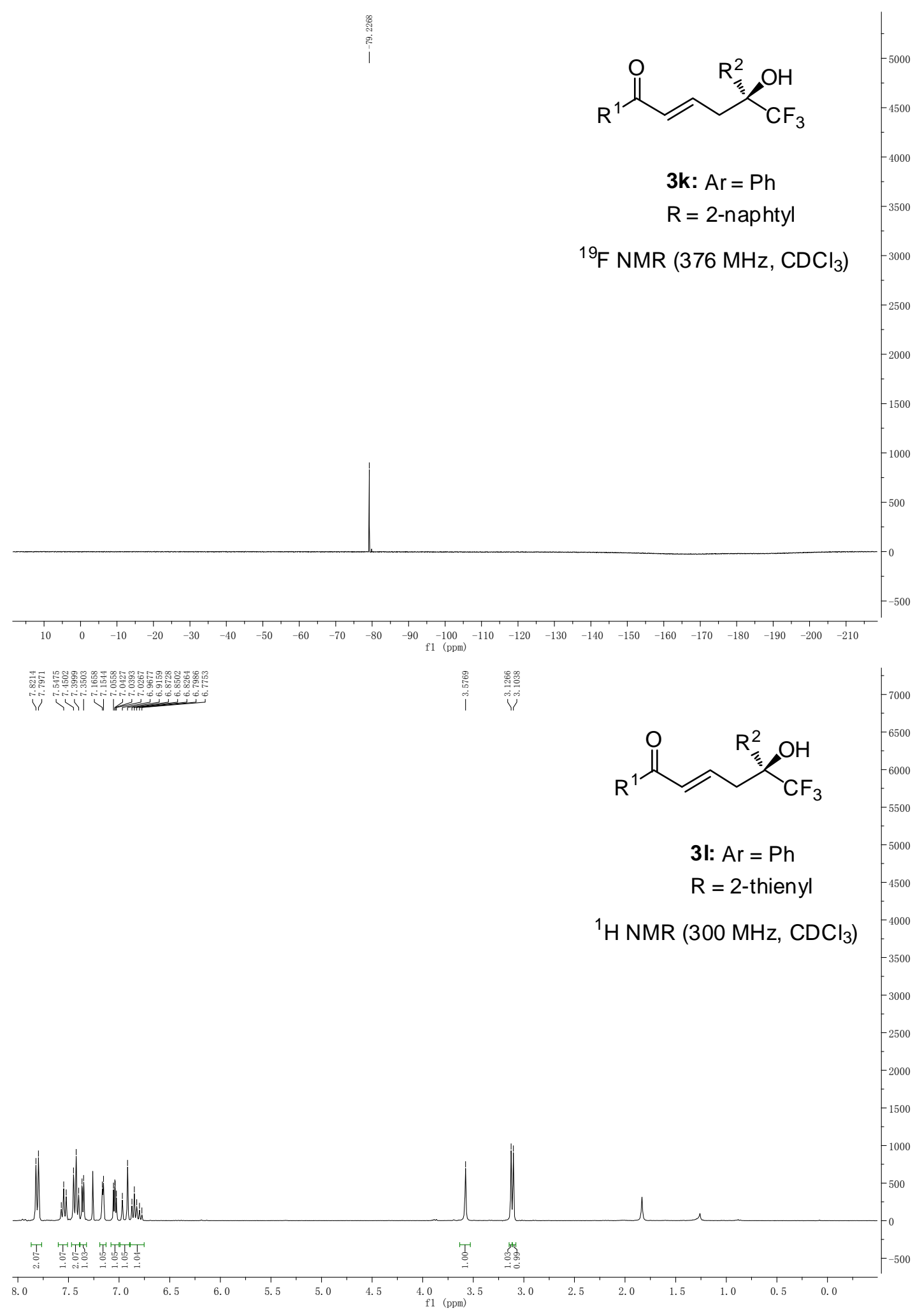




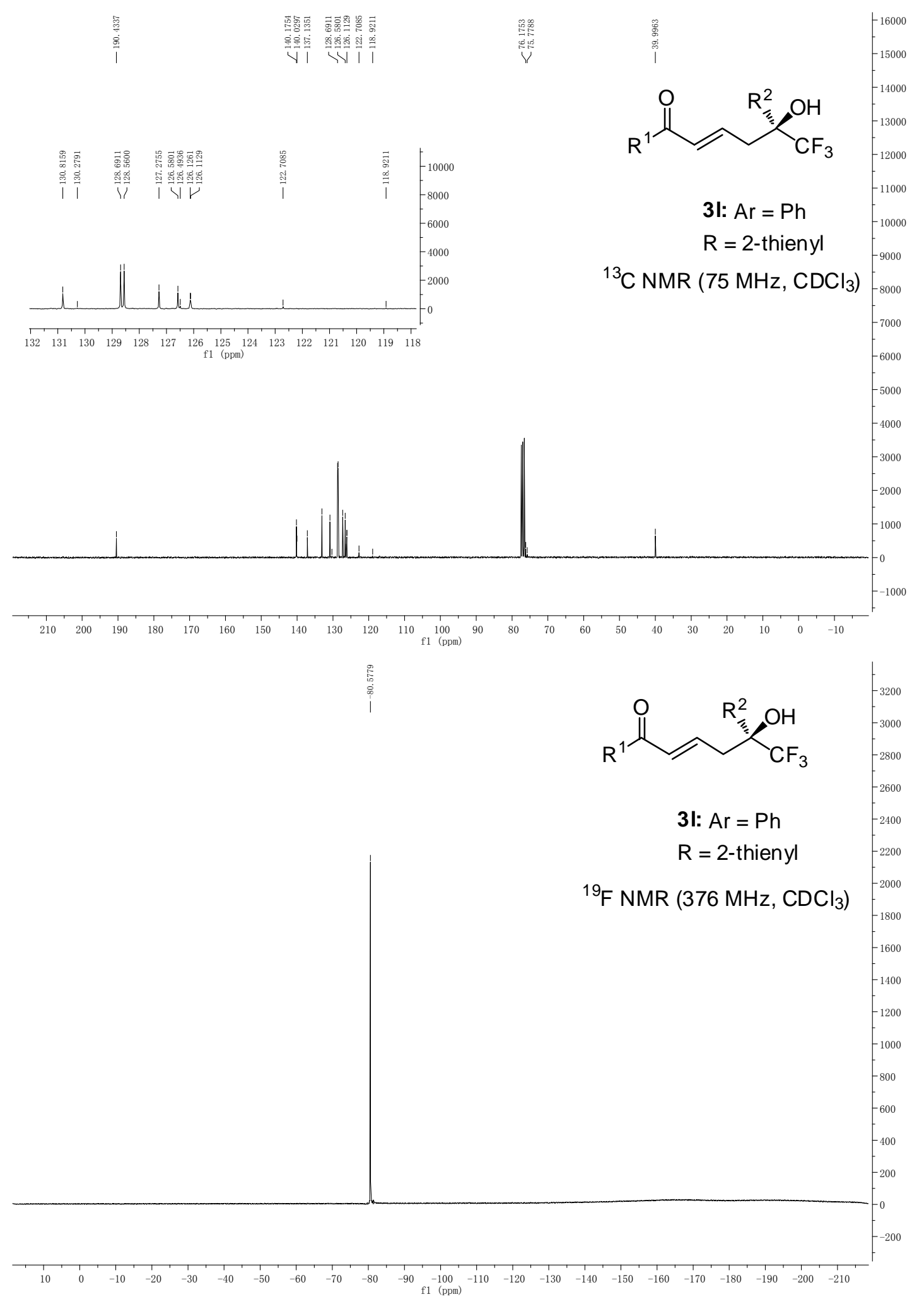




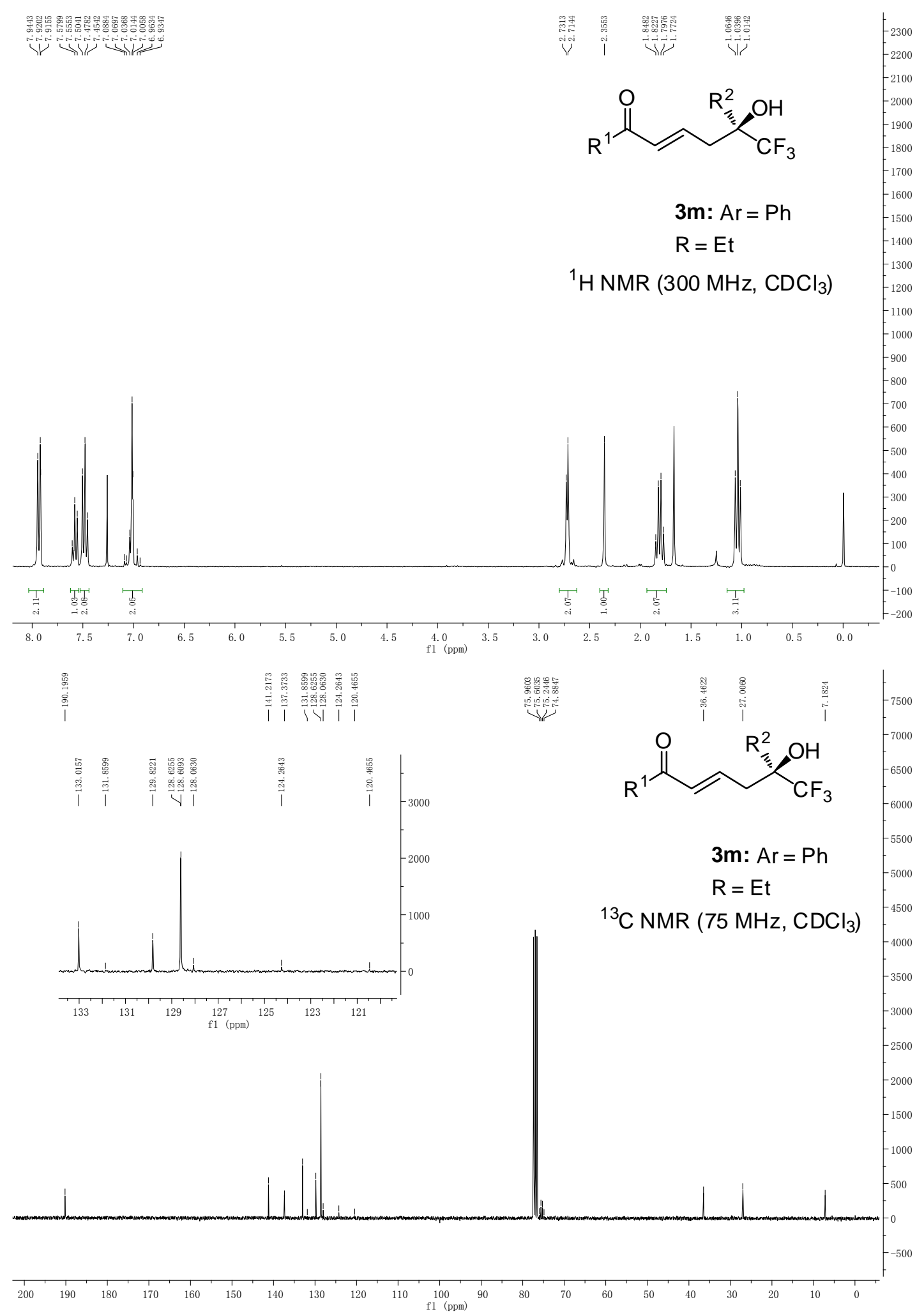




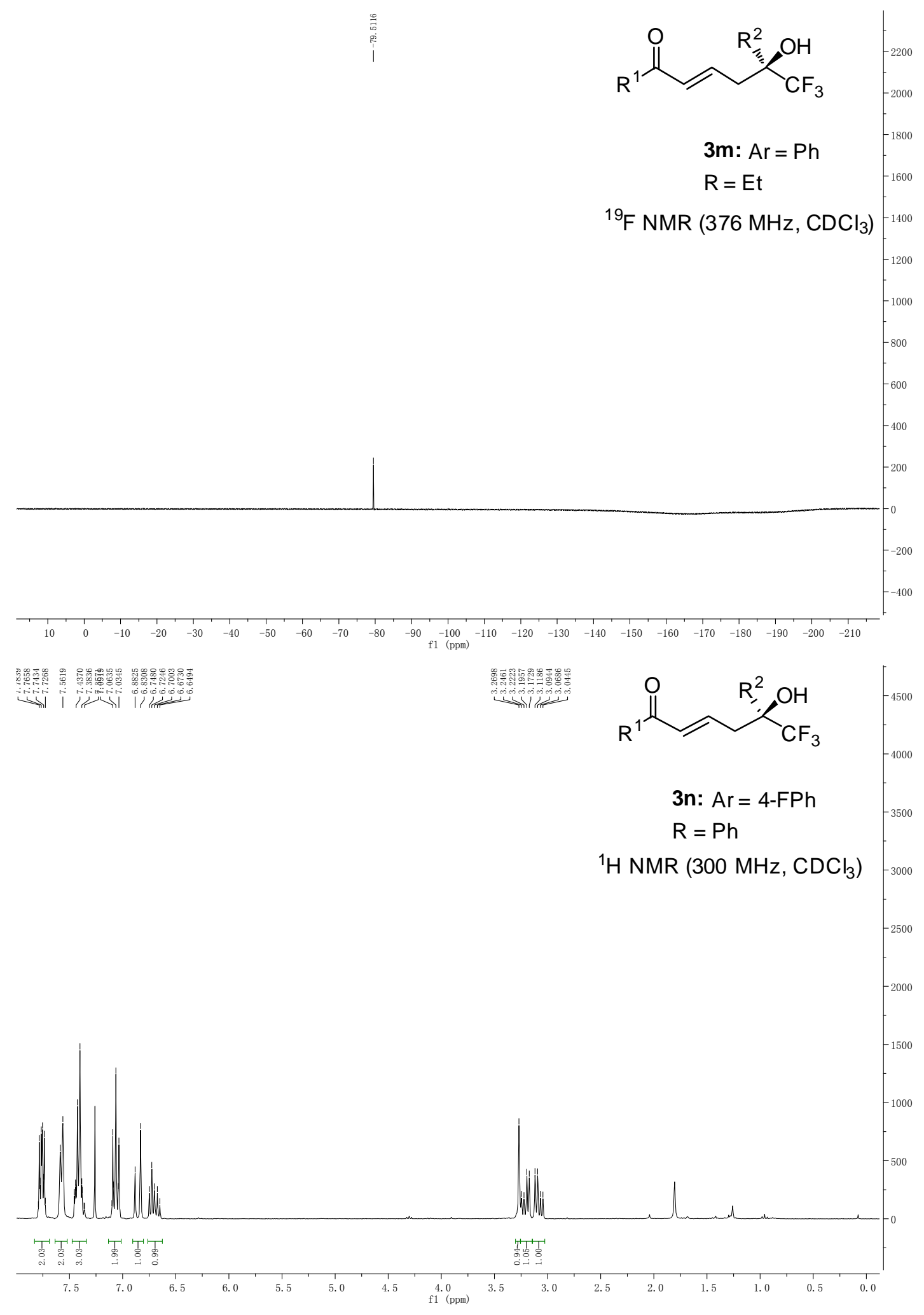




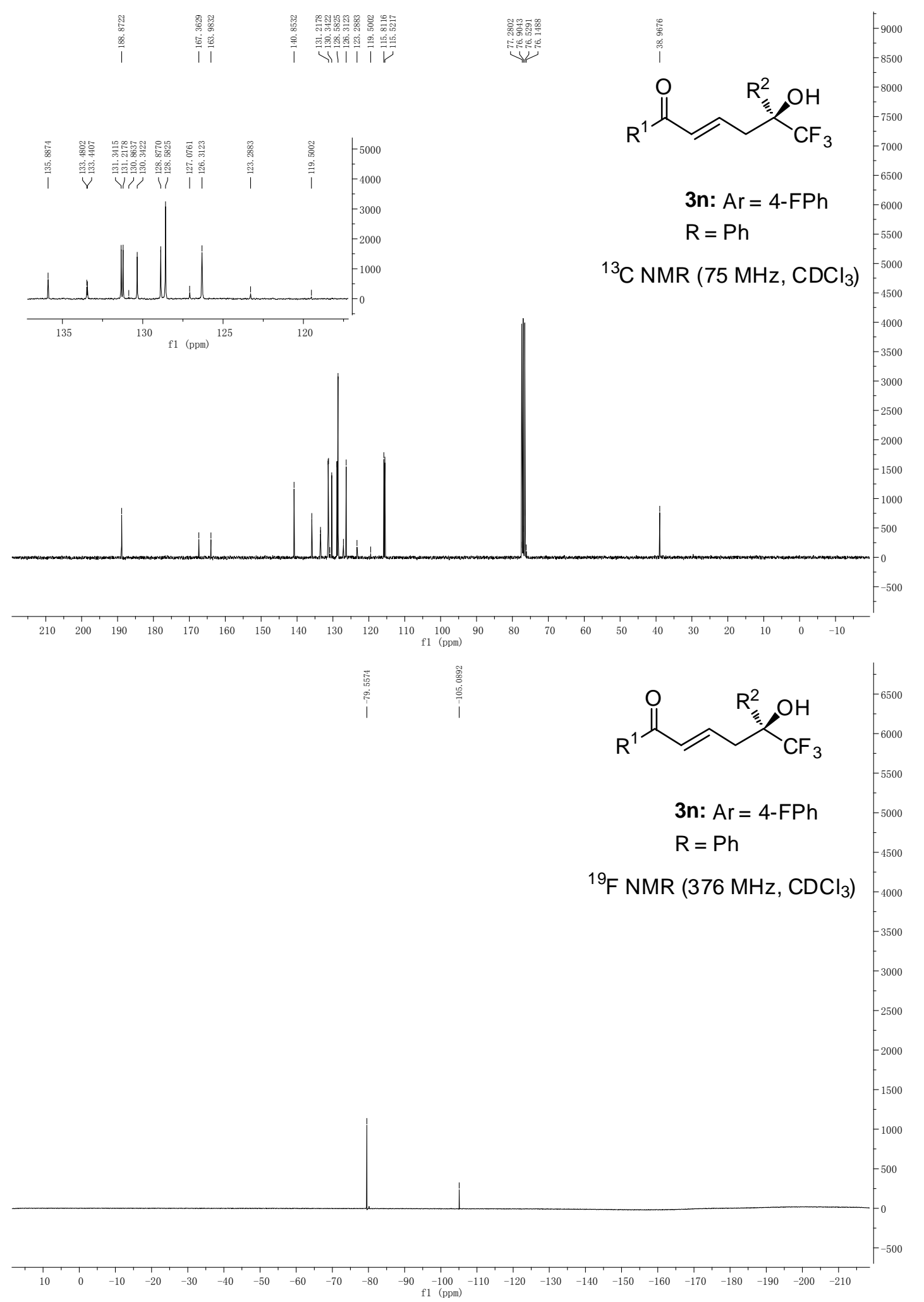




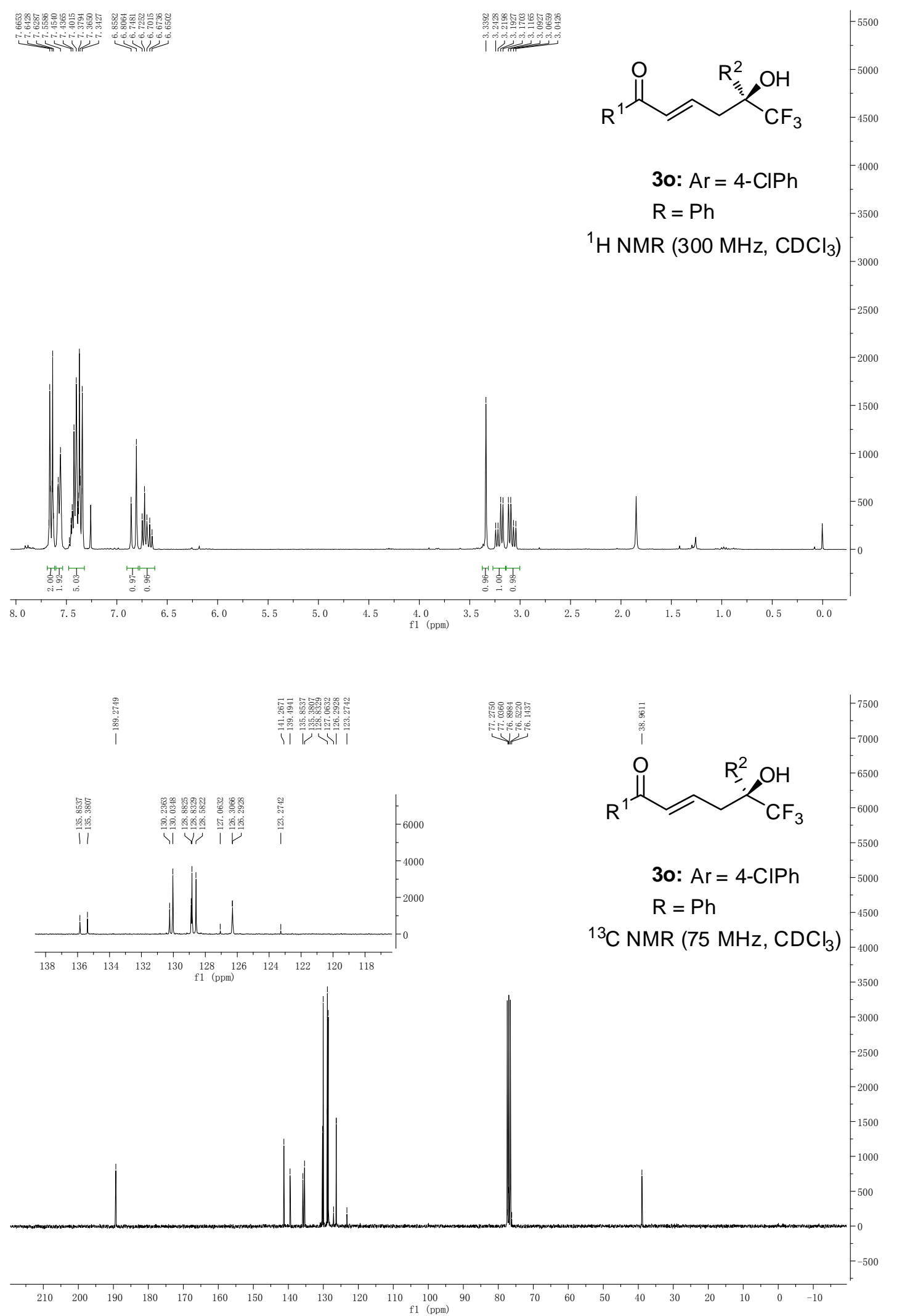




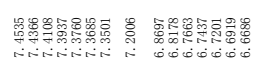

倠

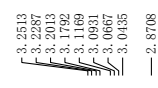

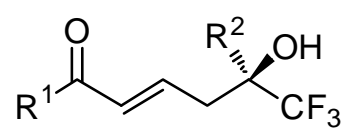

$-1900$

3p: Ar = 3-FPh

$\mathrm{R}=\mathrm{Ph}$

${ }^{1} \mathrm{H}$ NMR (300 MHz, $\left.\mathrm{CDCl}_{3}\right)$
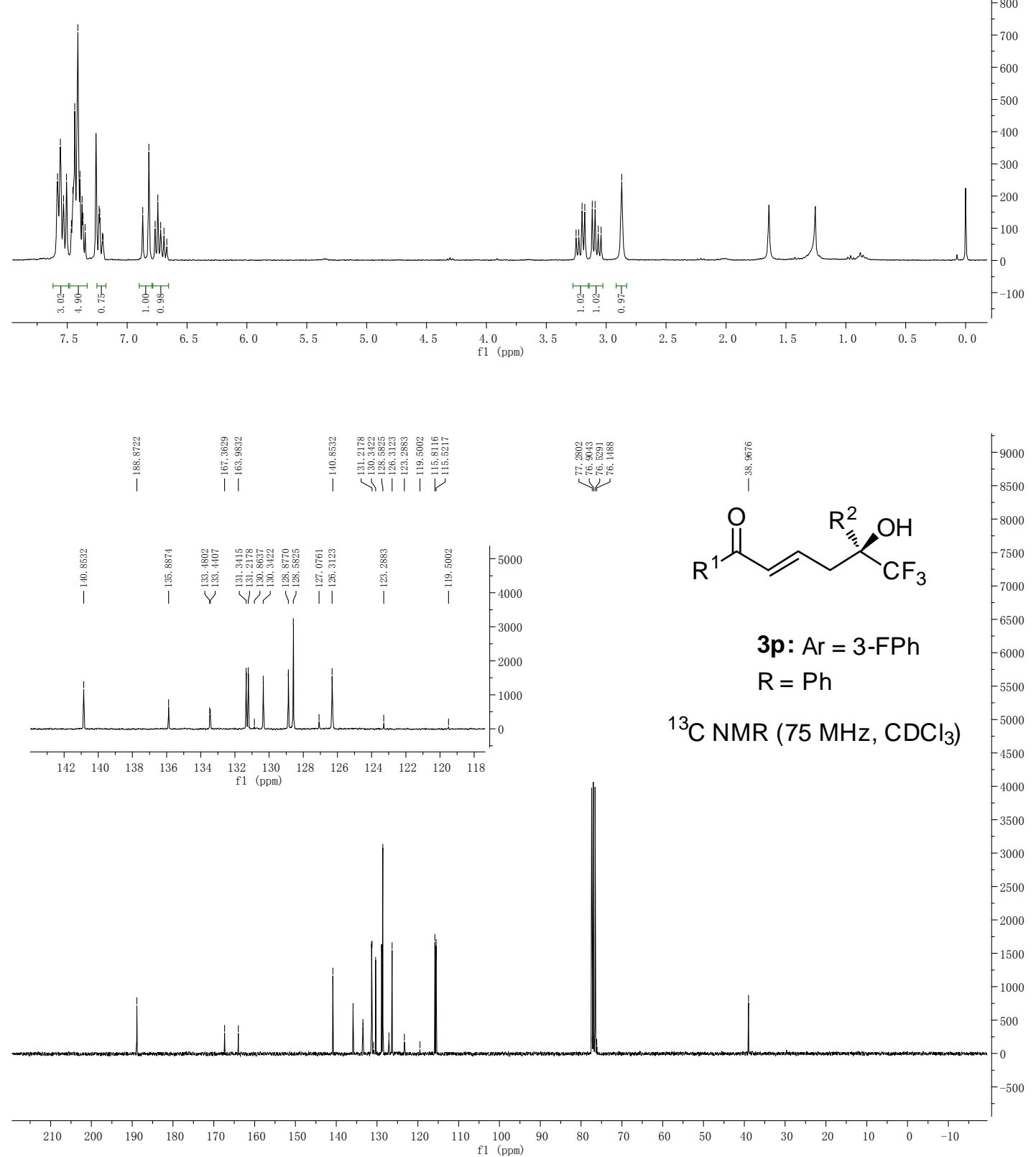


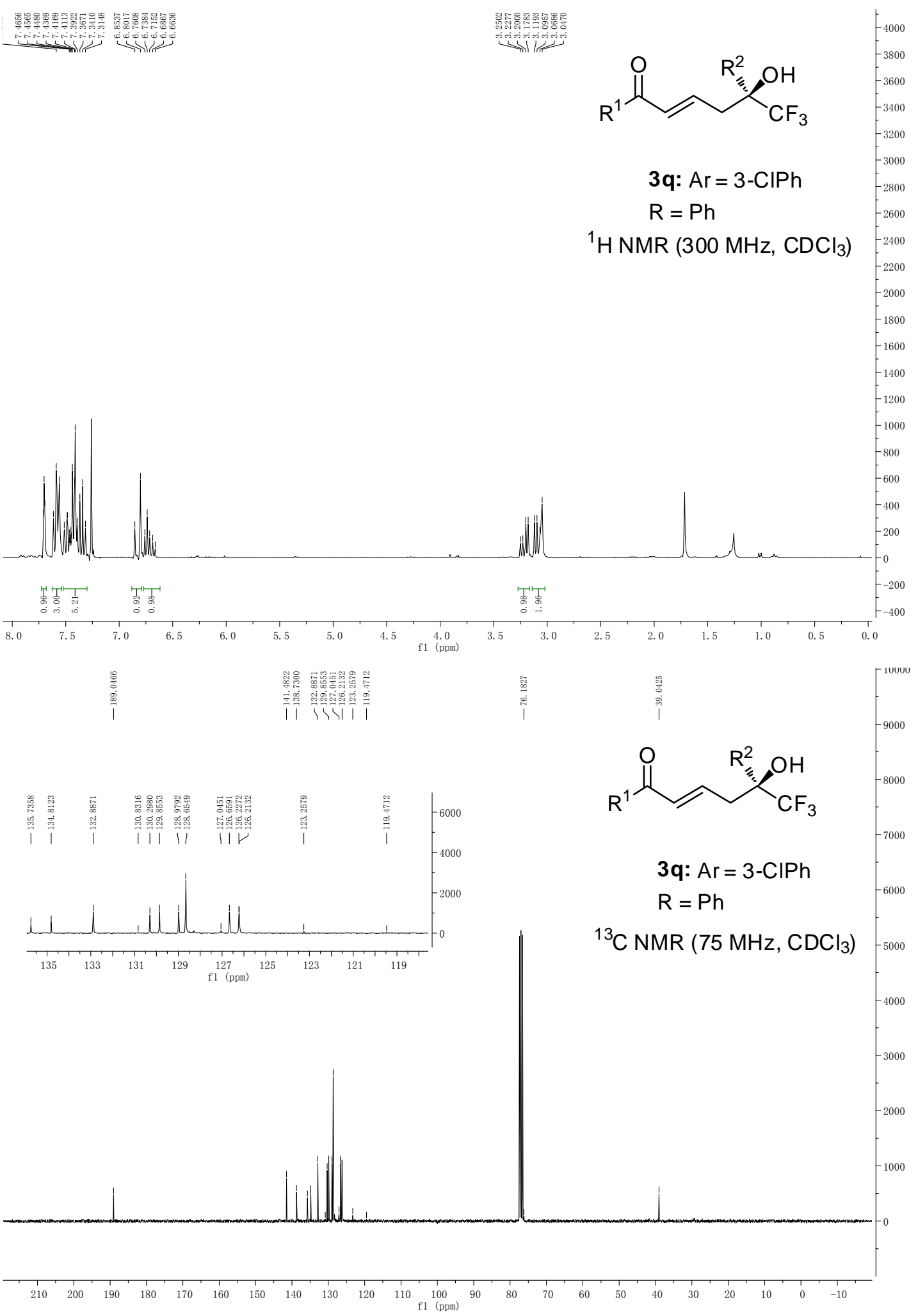




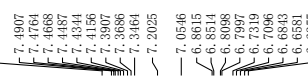

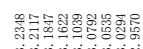

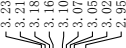<smiles>[R]C(=O)/C=C/CC([R2])(O)C(F)(F)F</smiles>

$\mathrm{R}=\mathrm{Ph}$

${ }^{1} \mathrm{H}$ NMR $\left(300 \mathrm{MHz}, \mathrm{CDCl}_{3}\right)$
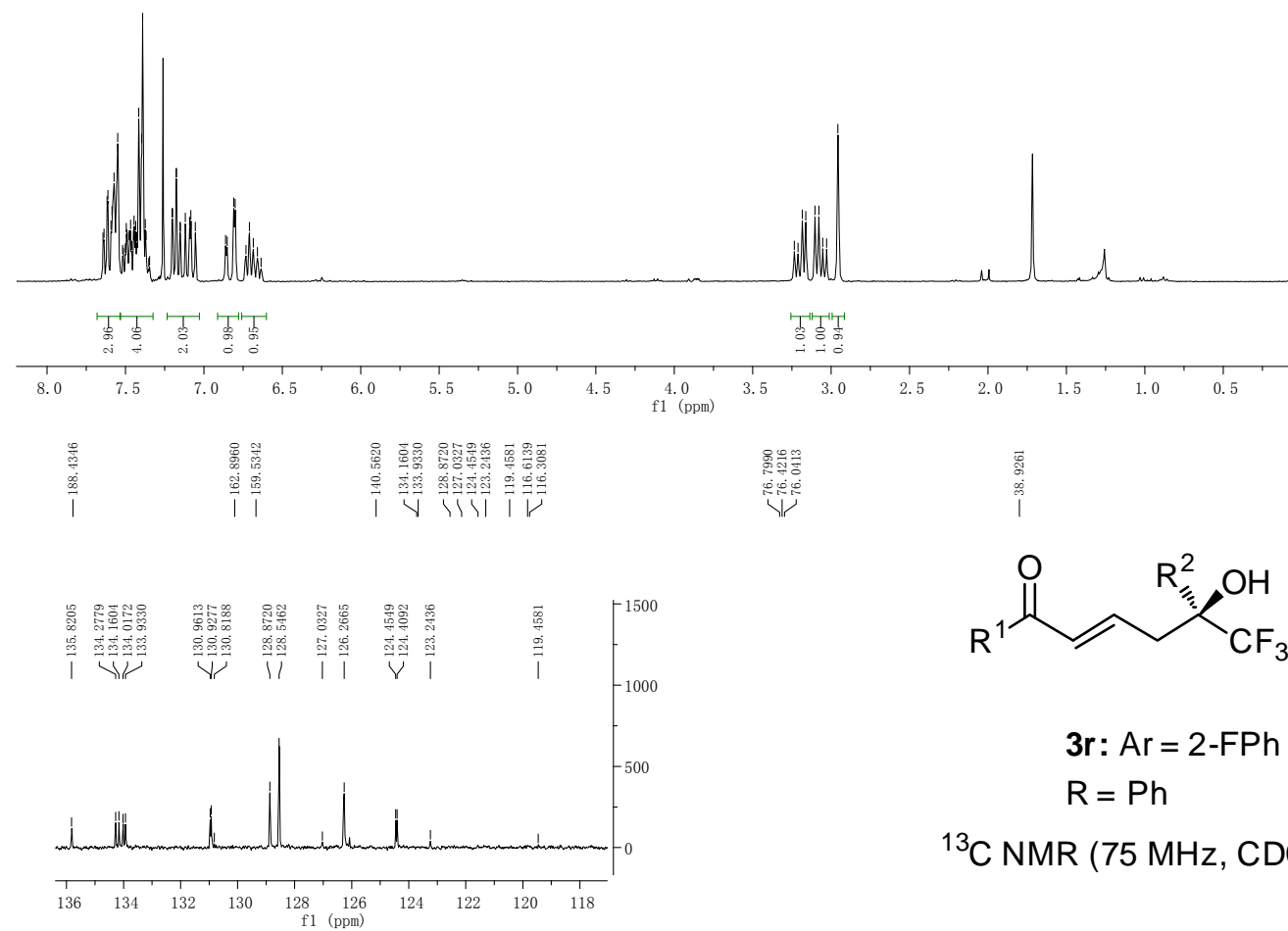

$3 r: A r=2-F P h$

$\mathrm{R}=\mathrm{Ph}$

${ }^{13} \mathrm{C}$ NMR $\left(75 \mathrm{MHz}, \mathrm{CDCl}_{3}\right)$

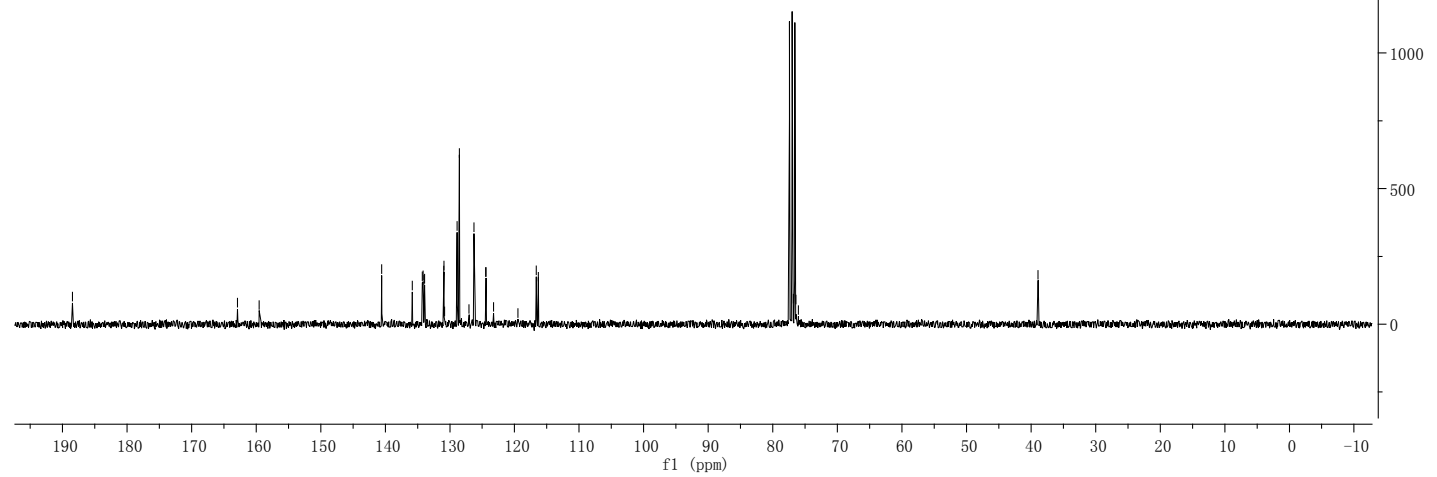




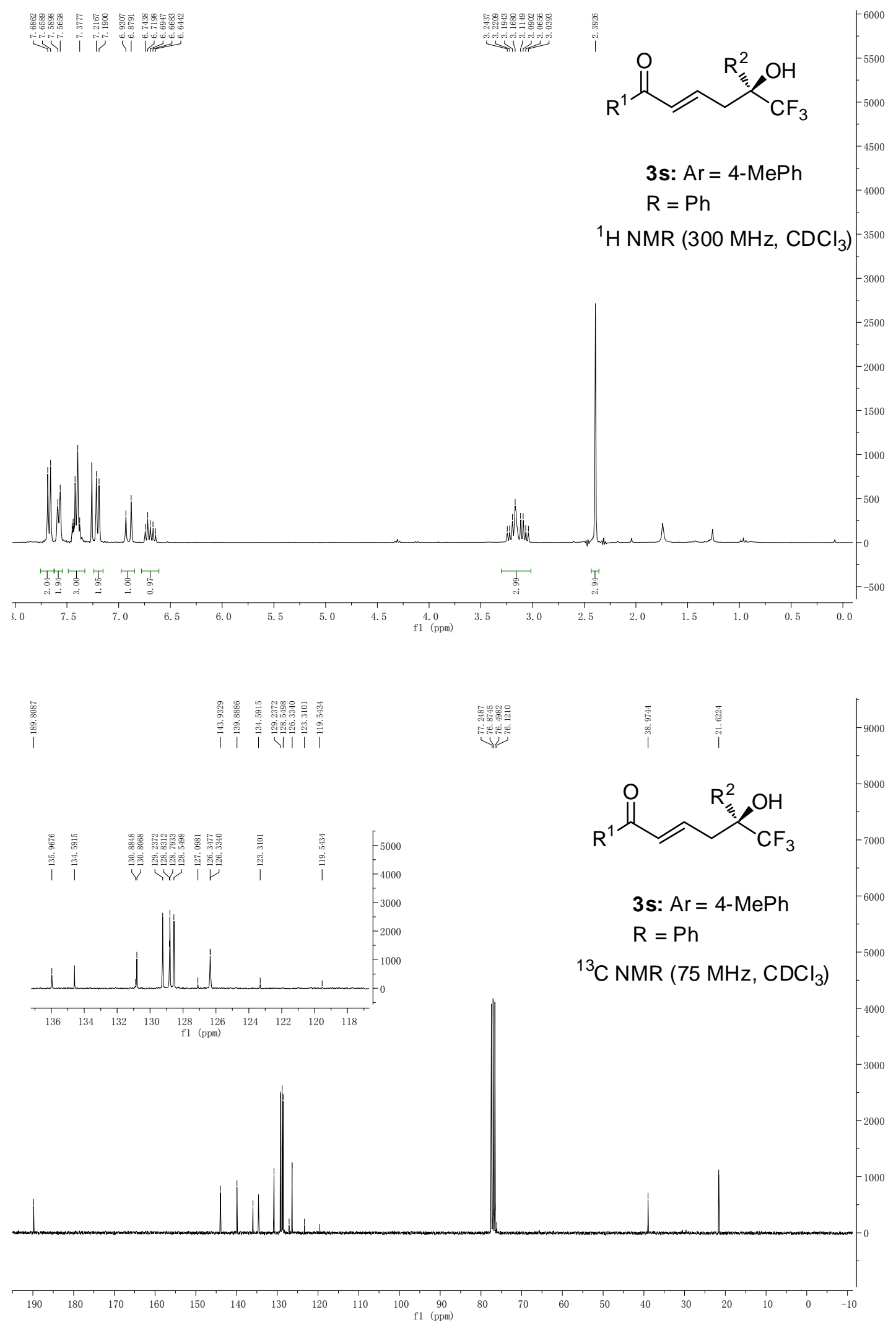




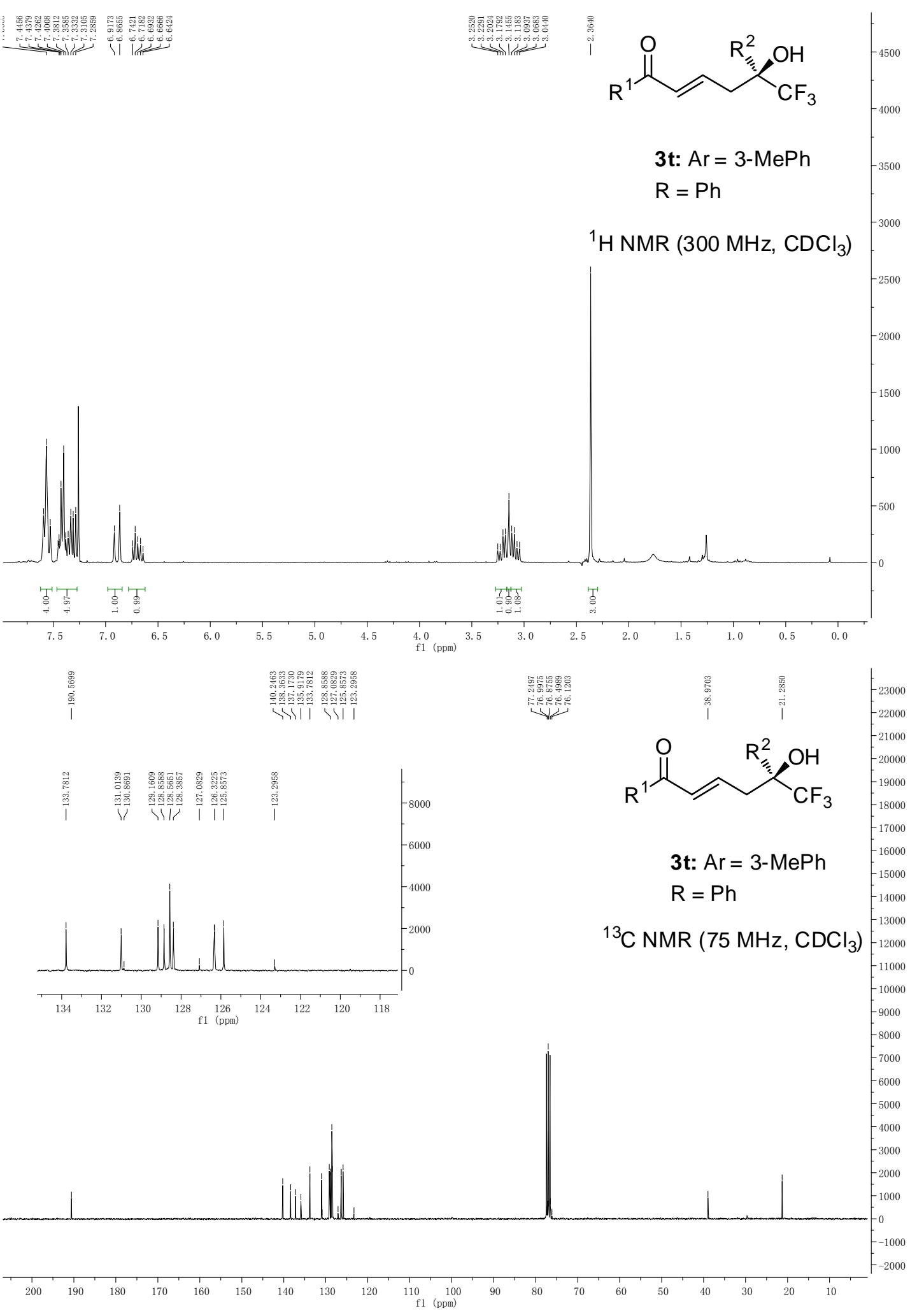




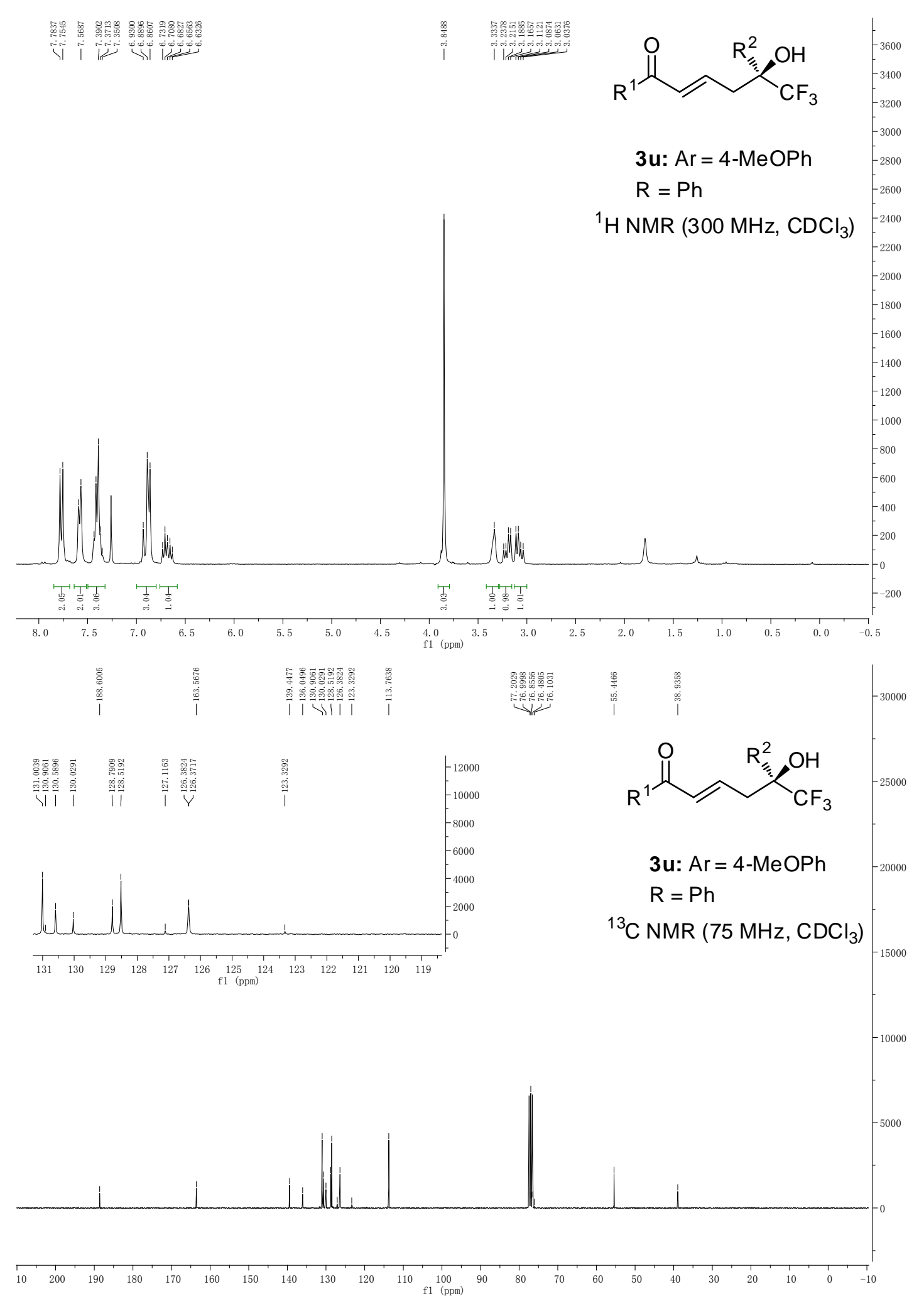



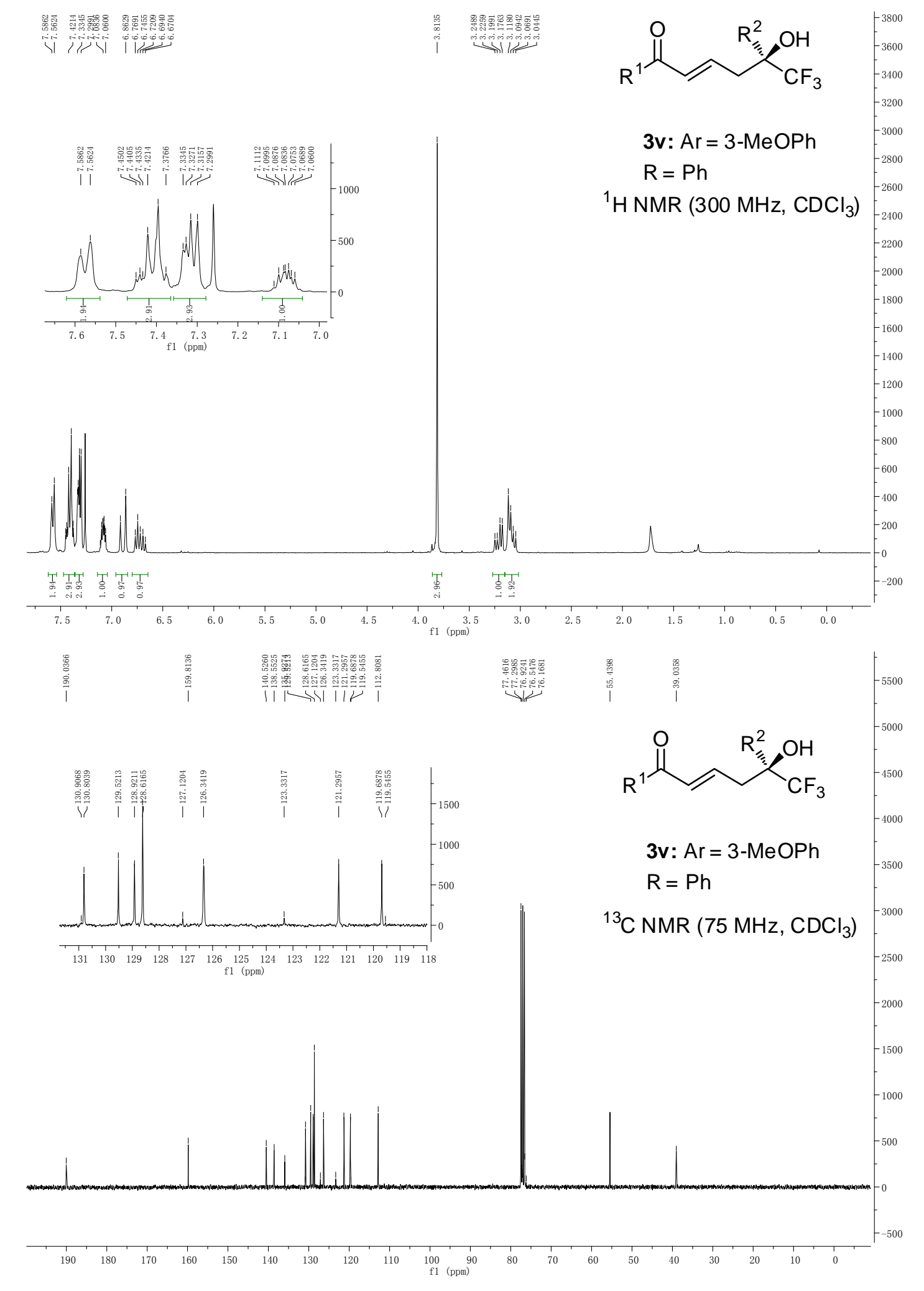


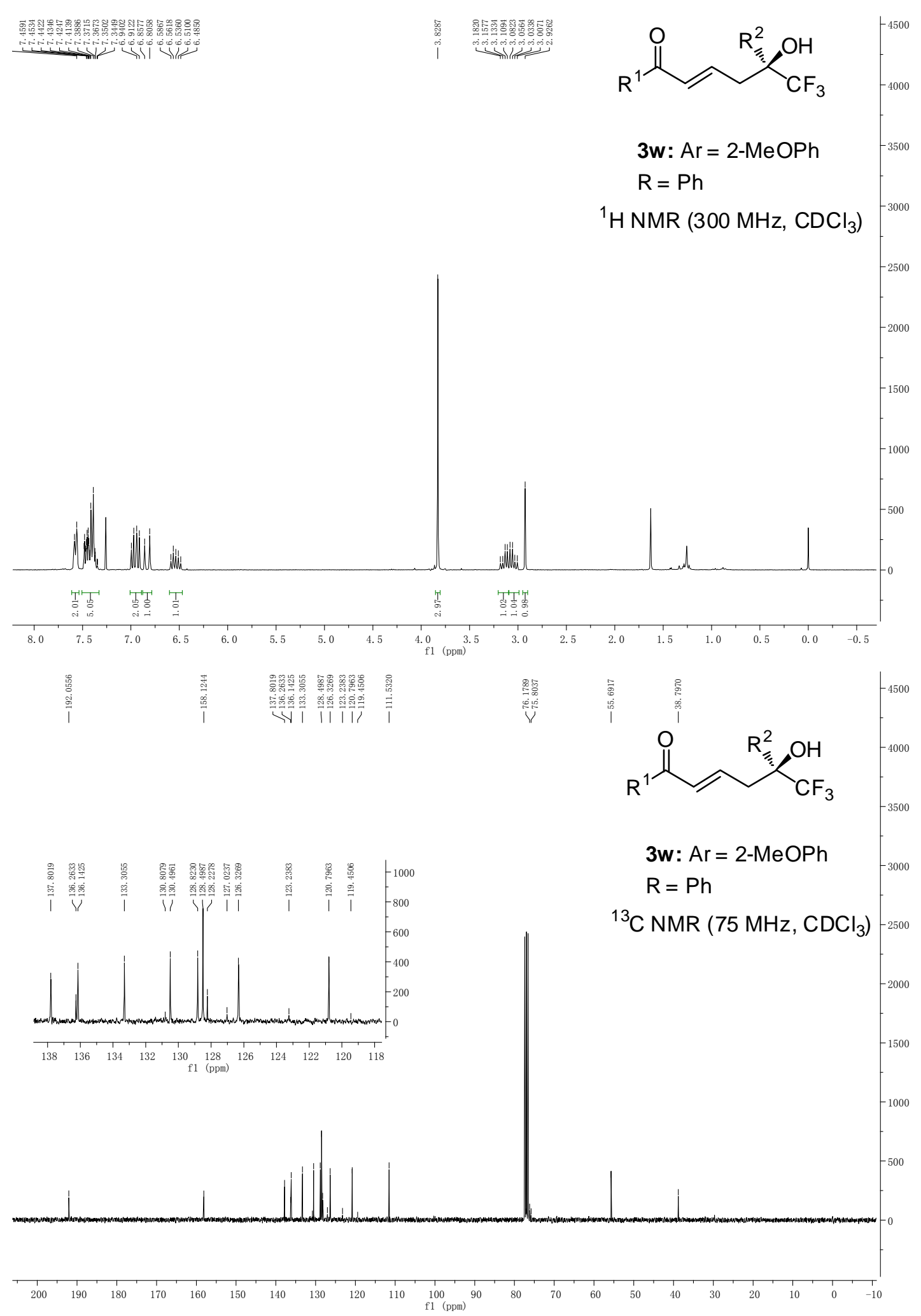




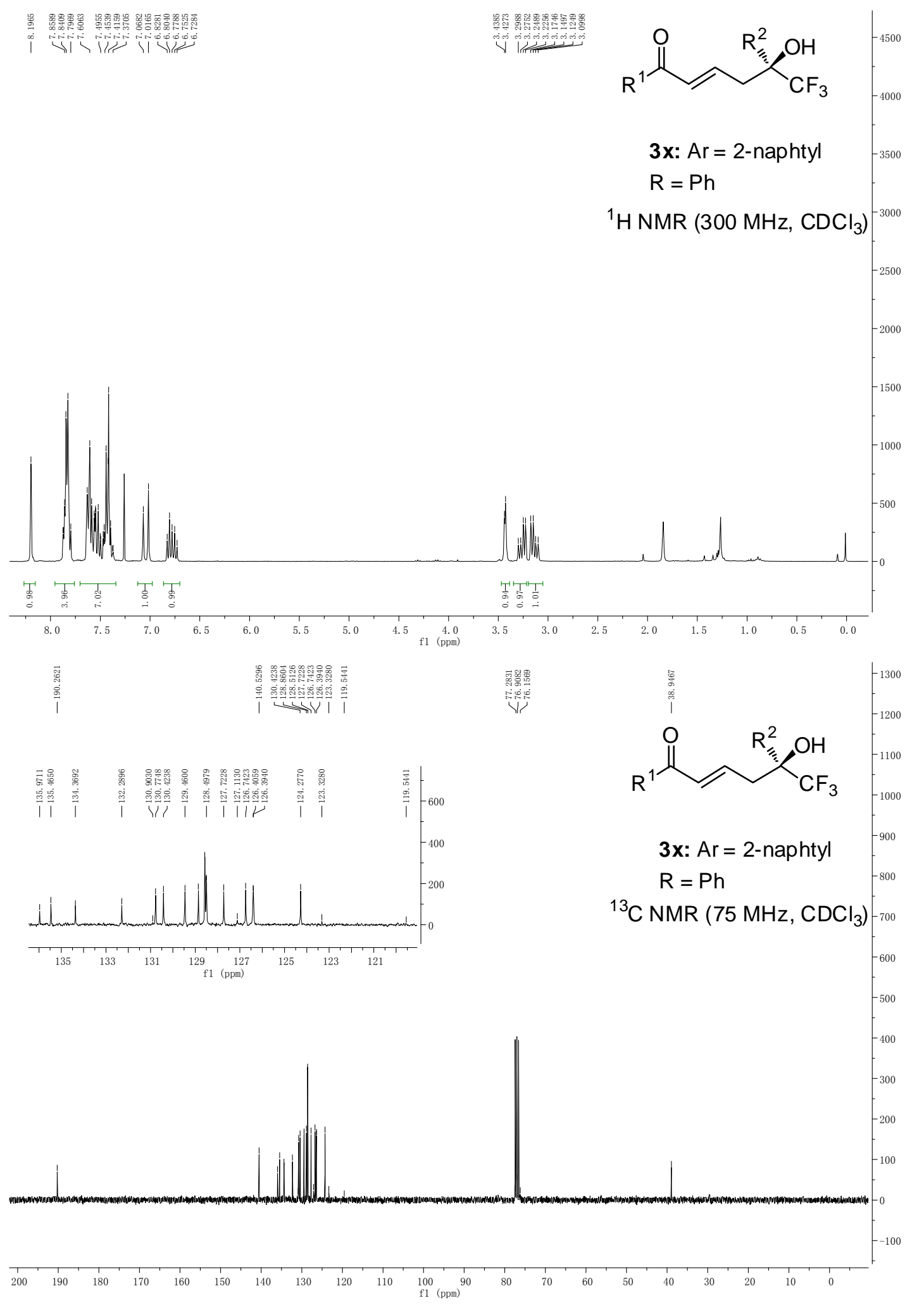




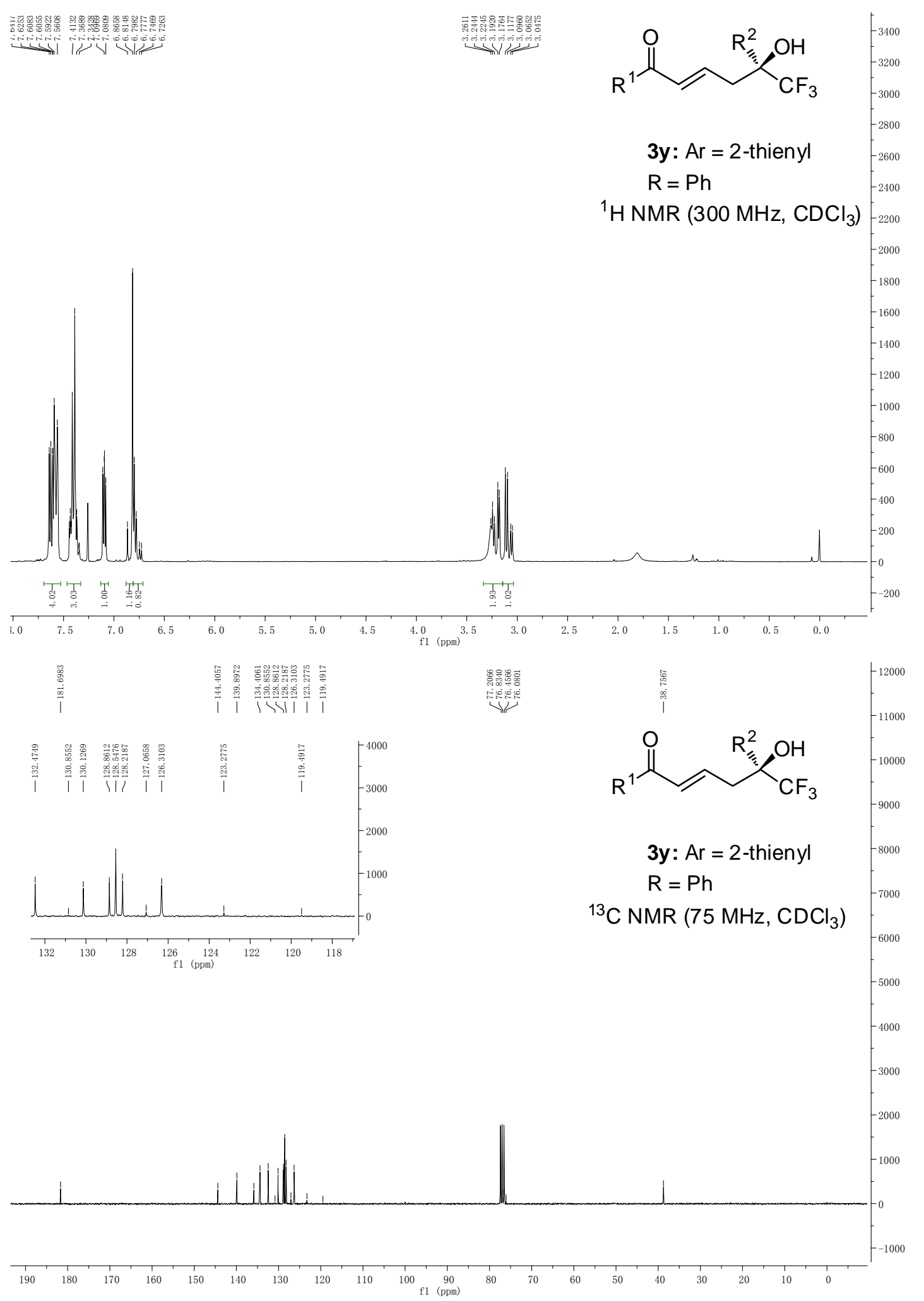



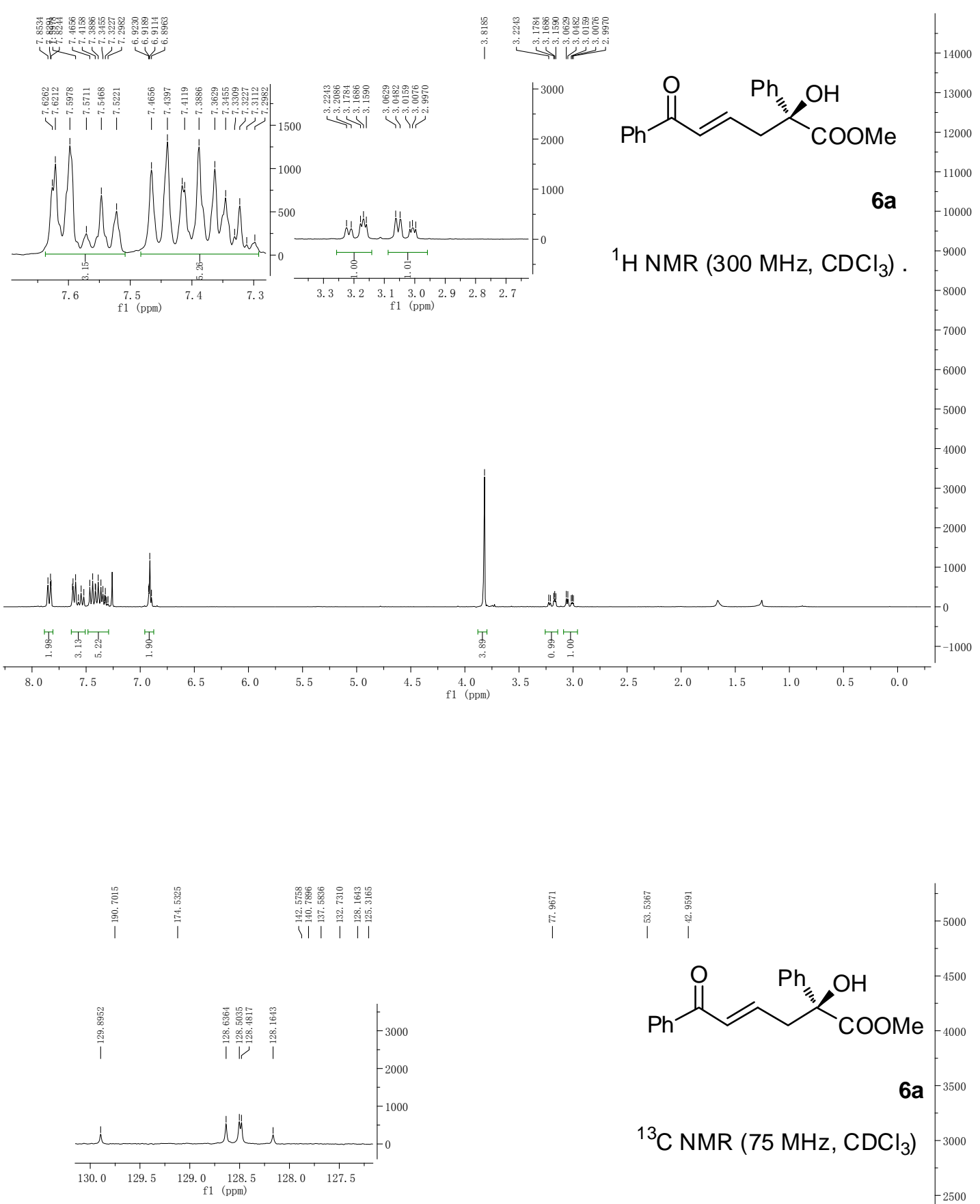

${ }^{13} \mathrm{C}$ NMR $\left(75 \mathrm{MHz}, \mathrm{CDCl}_{3}\right)$

3) 3000

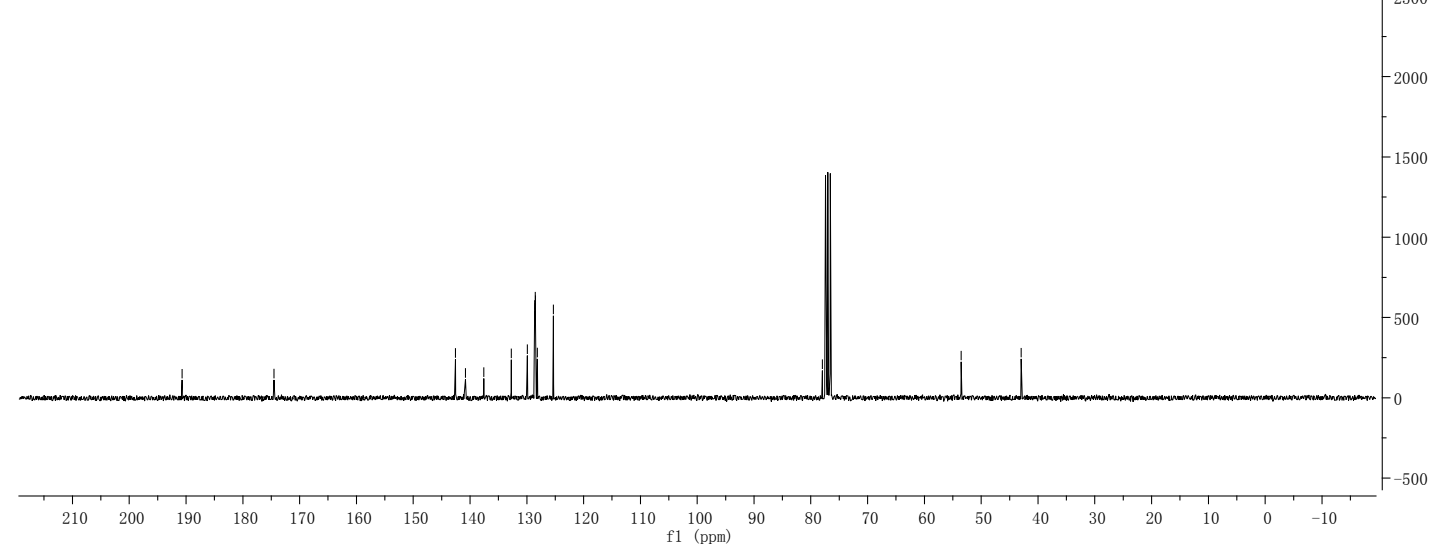




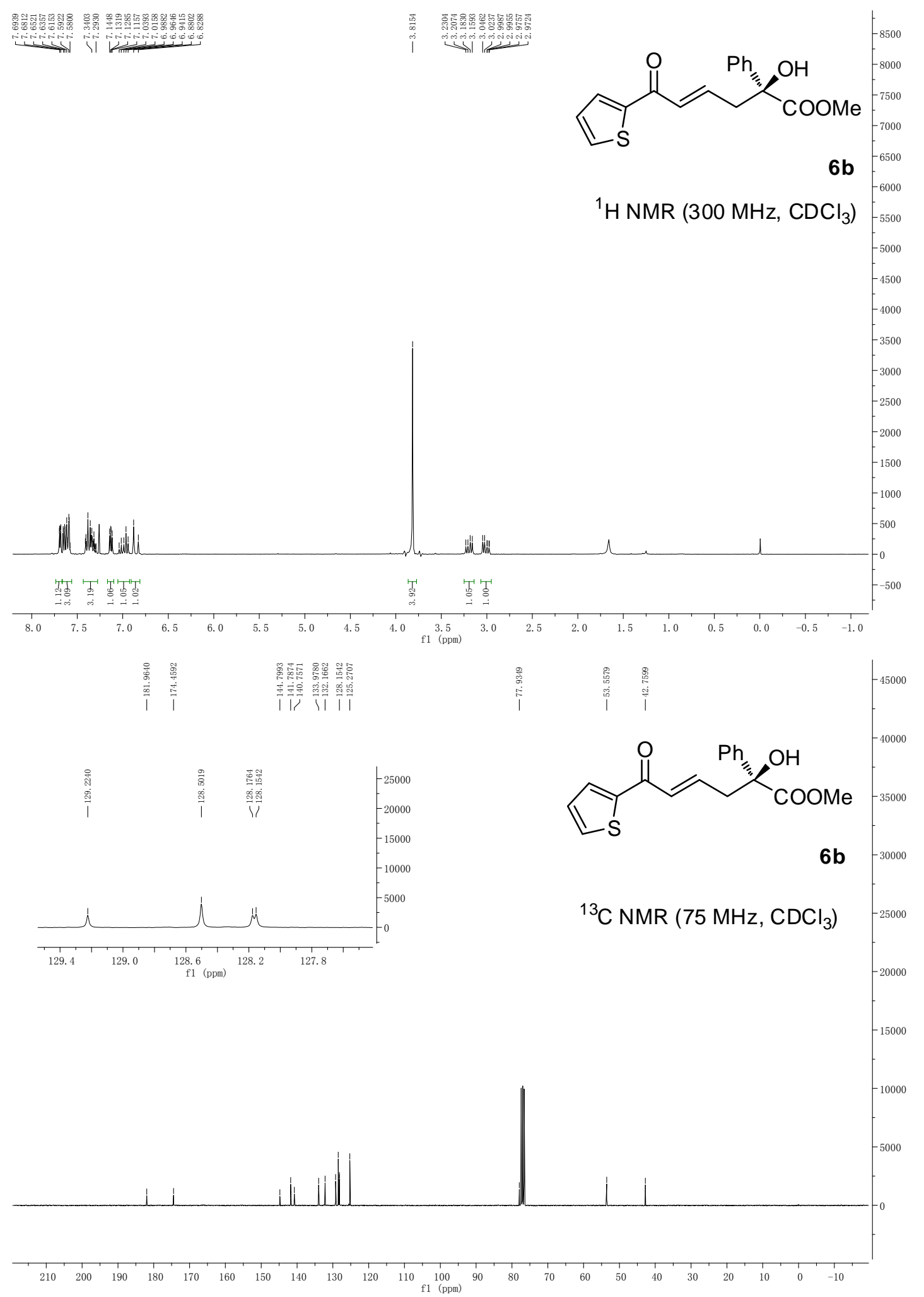




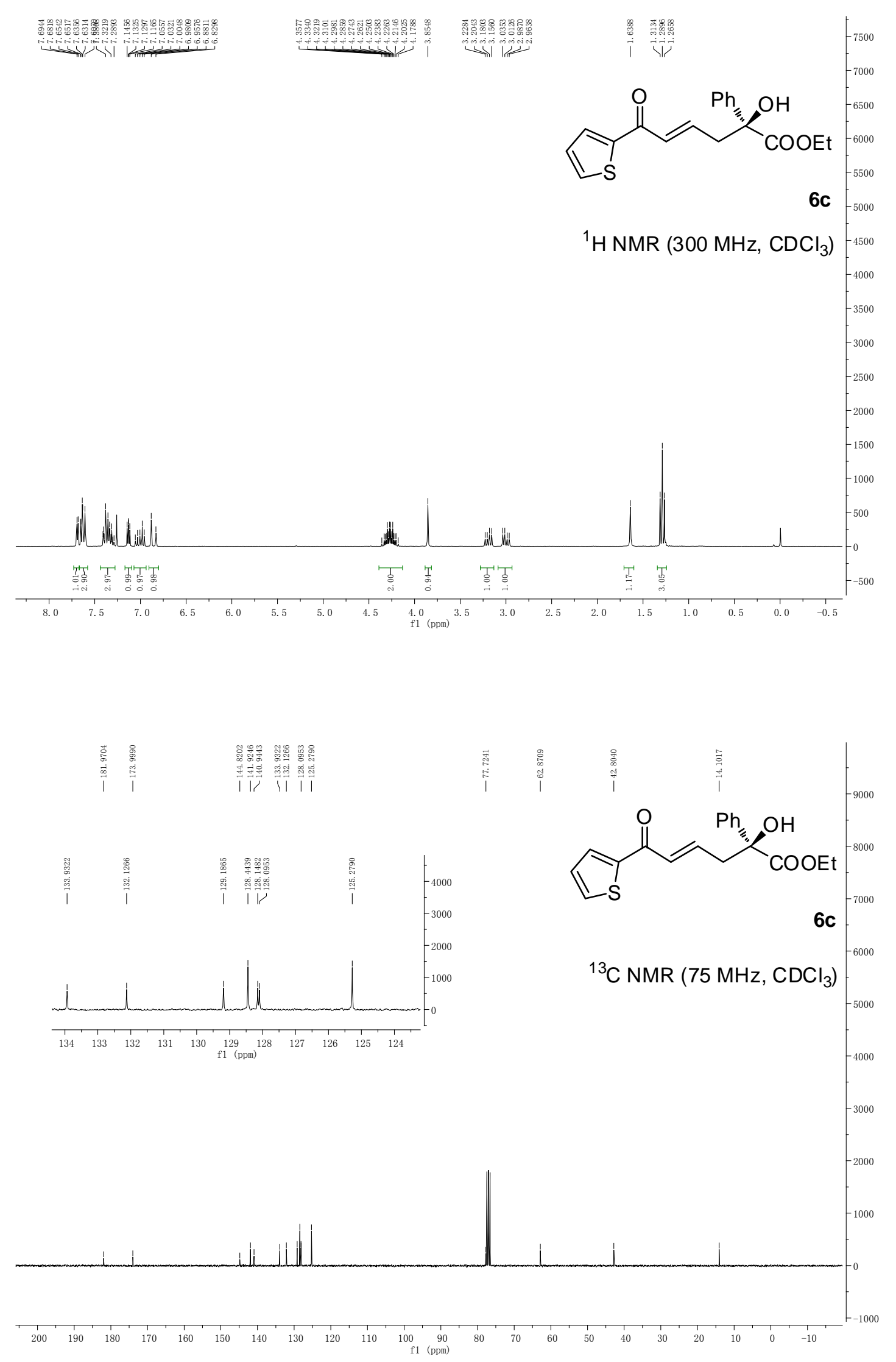




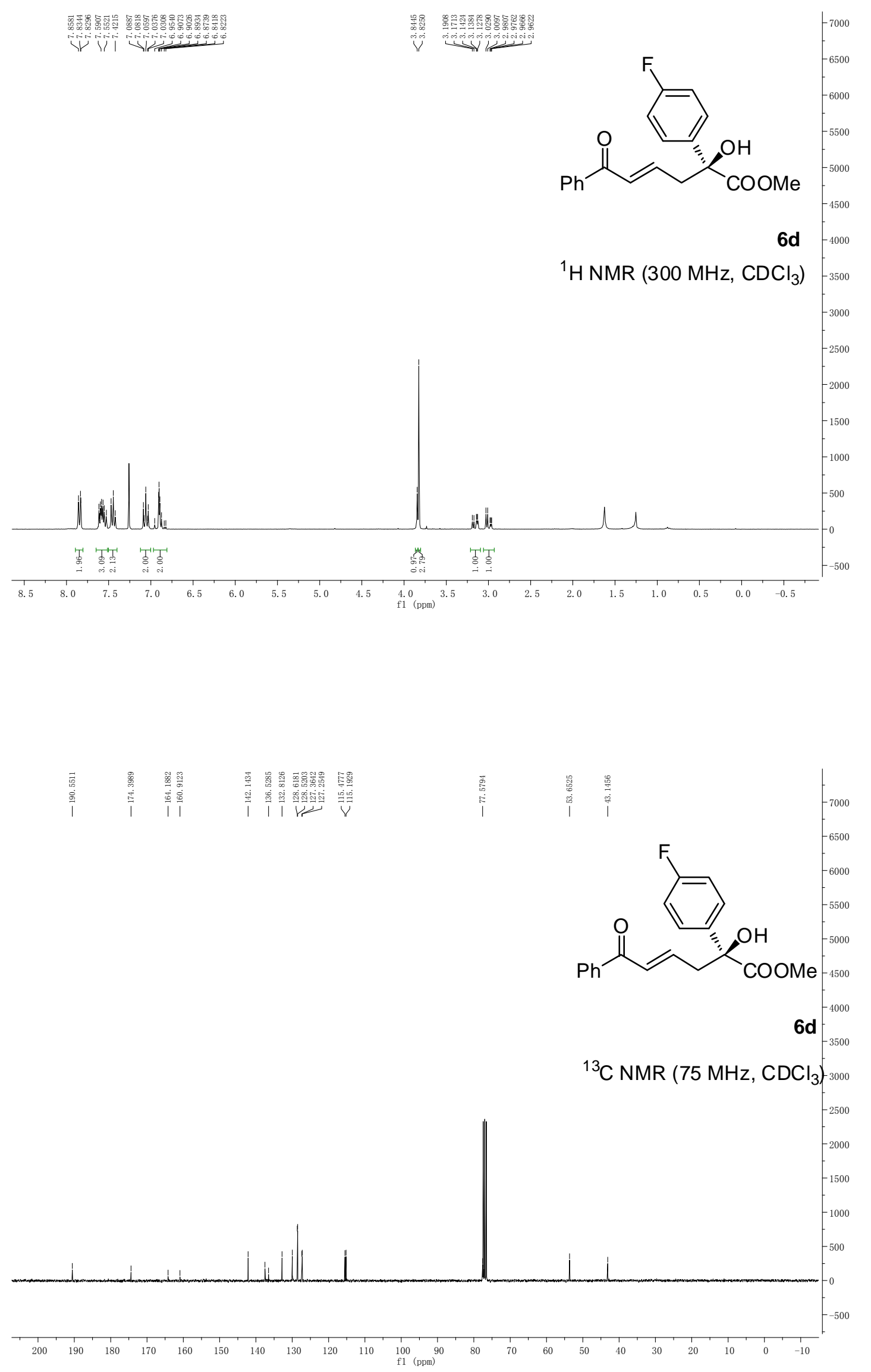




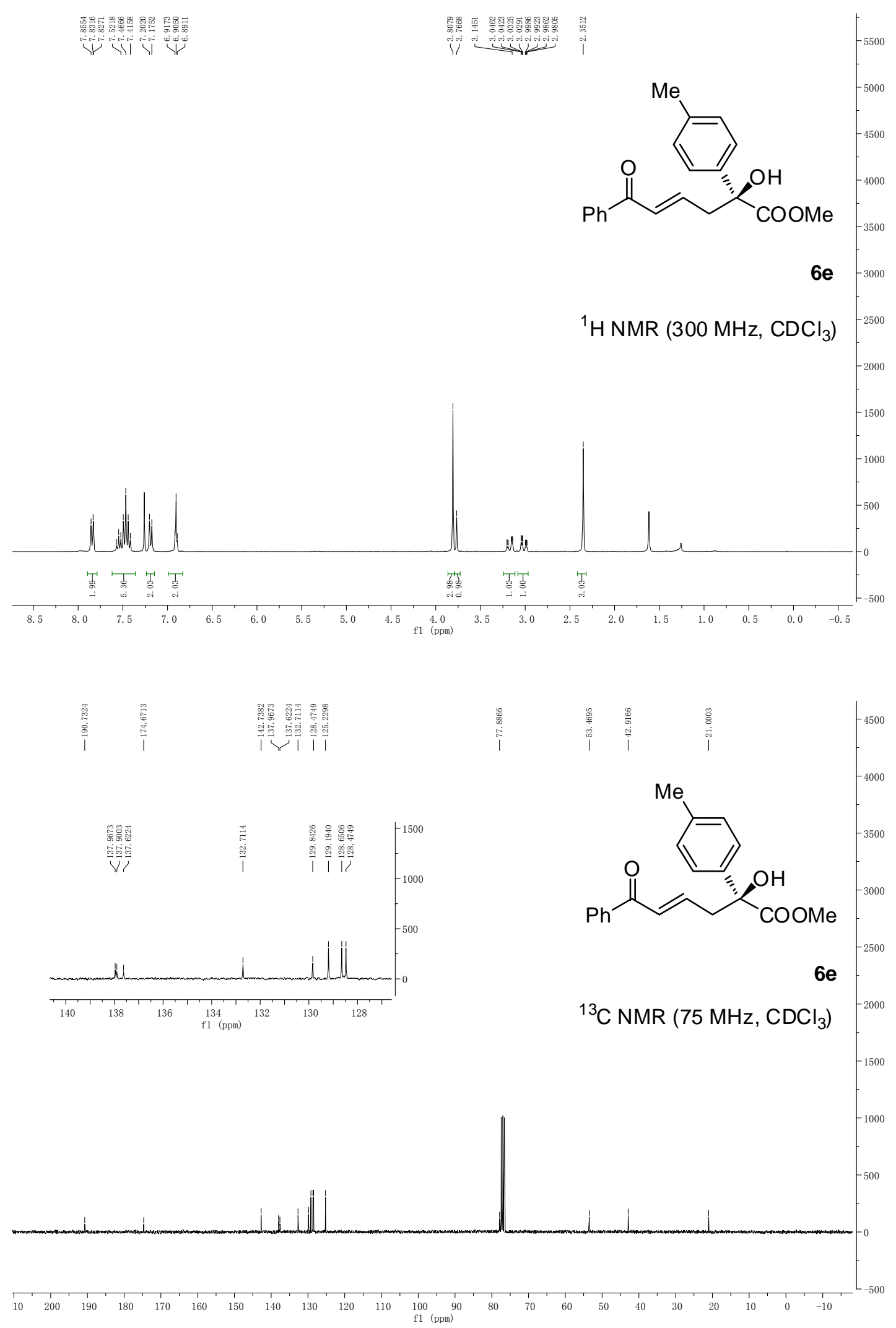



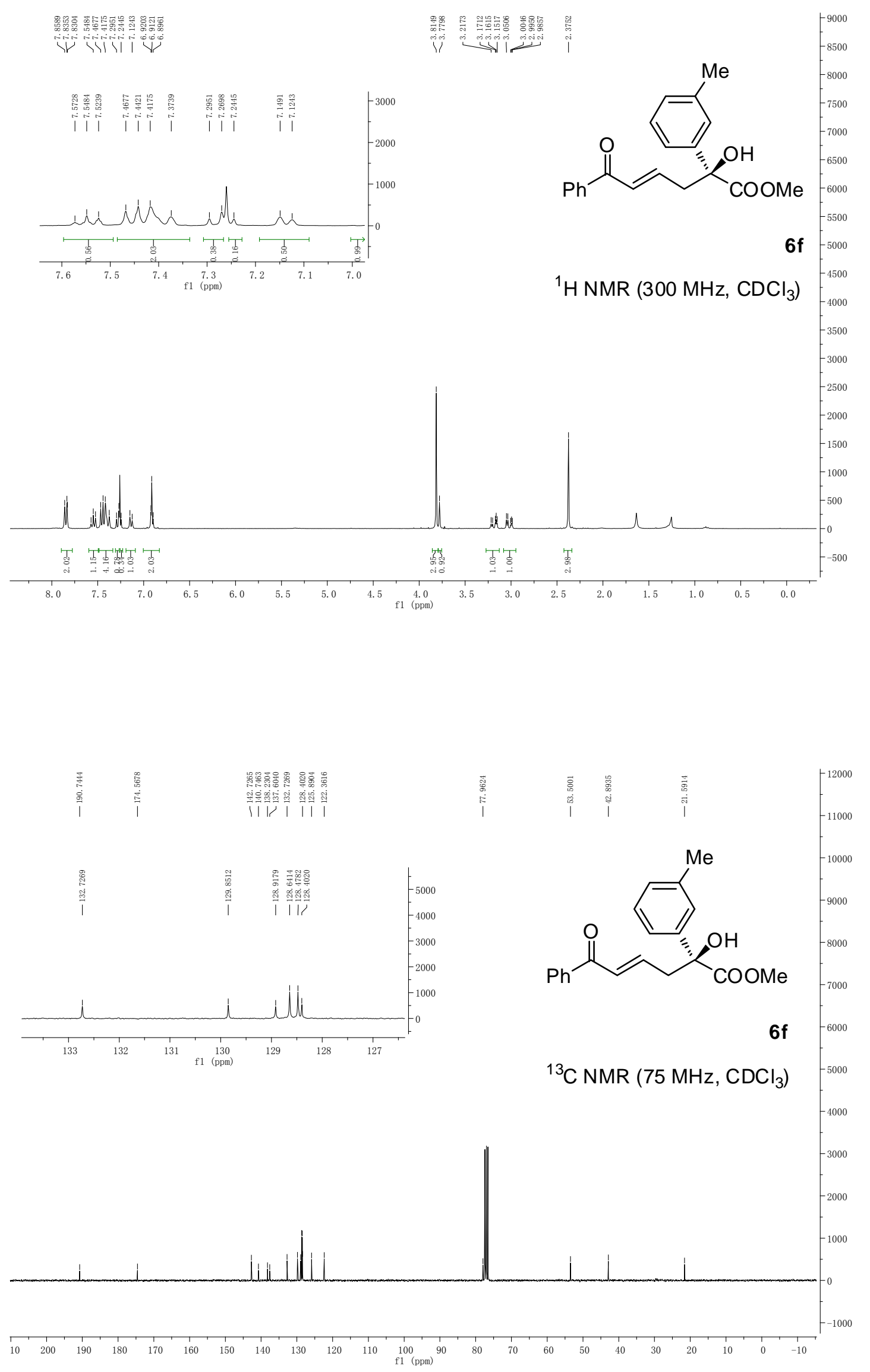


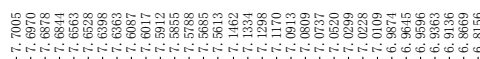

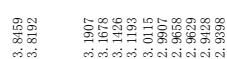
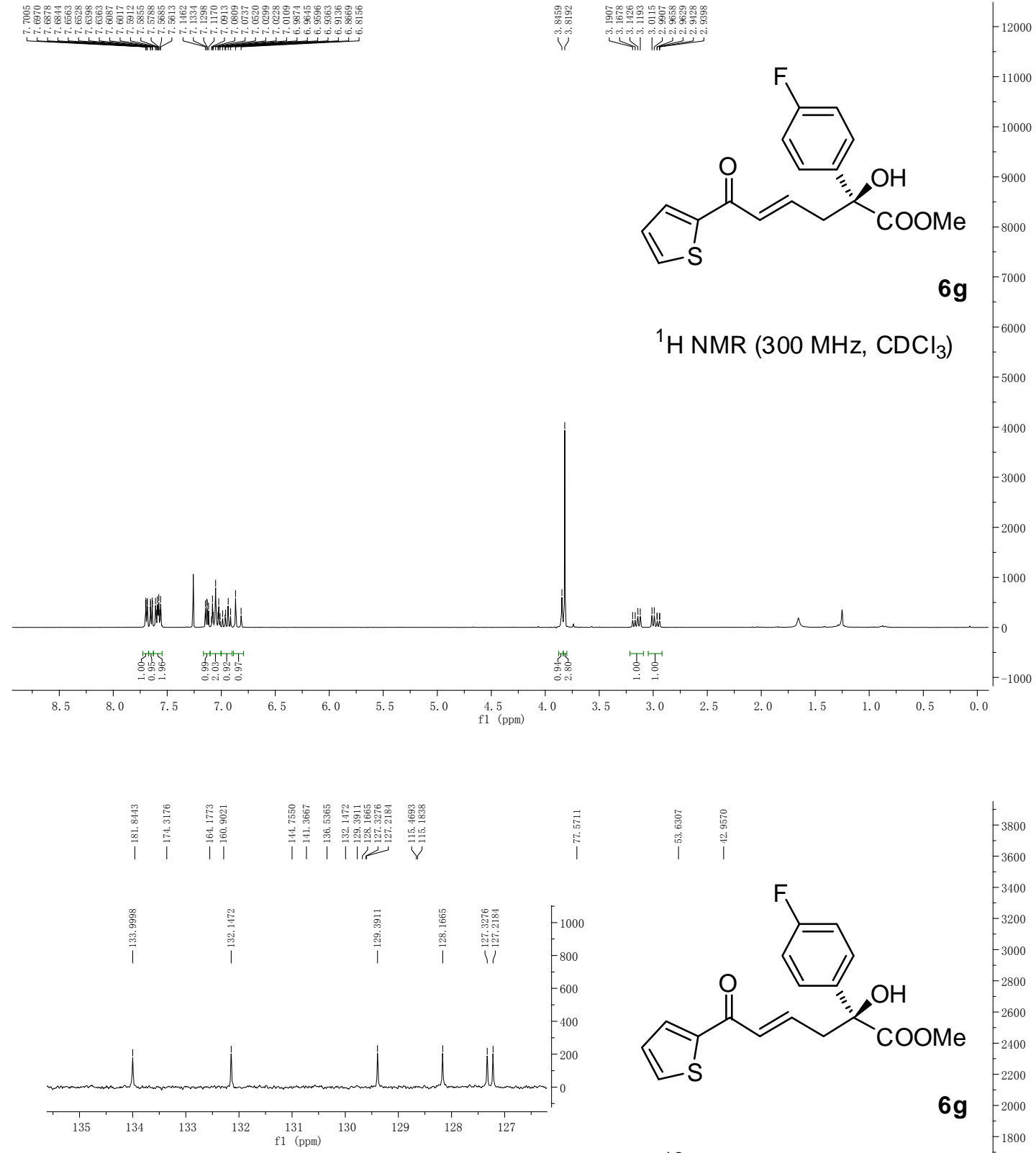

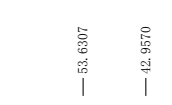

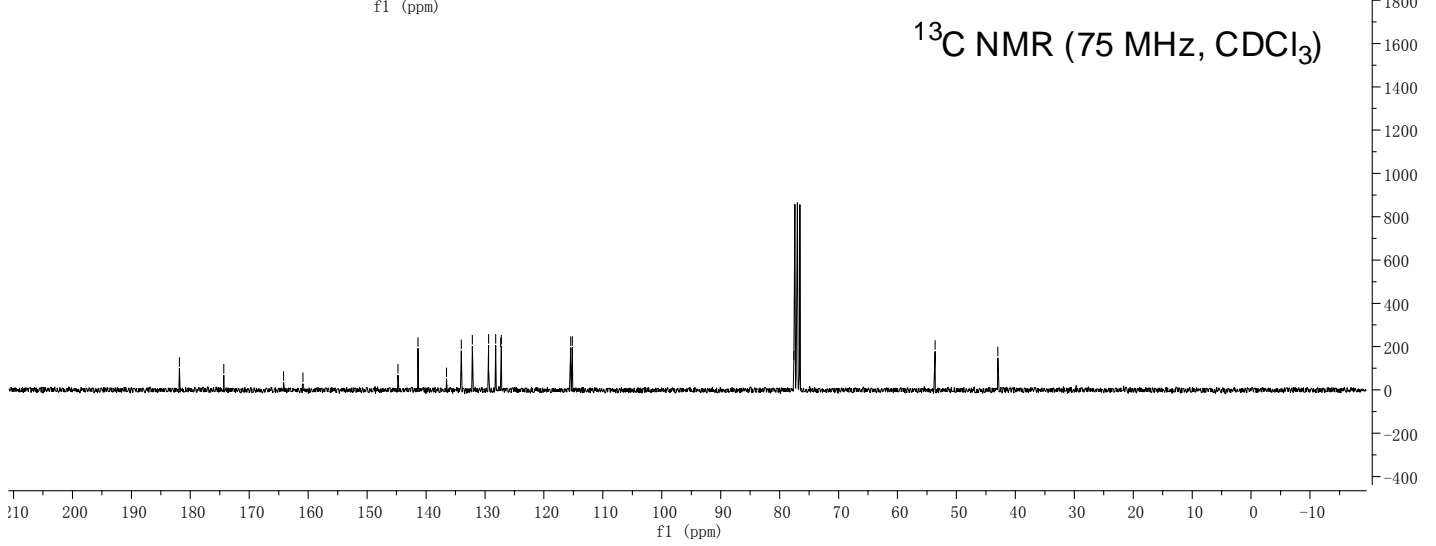




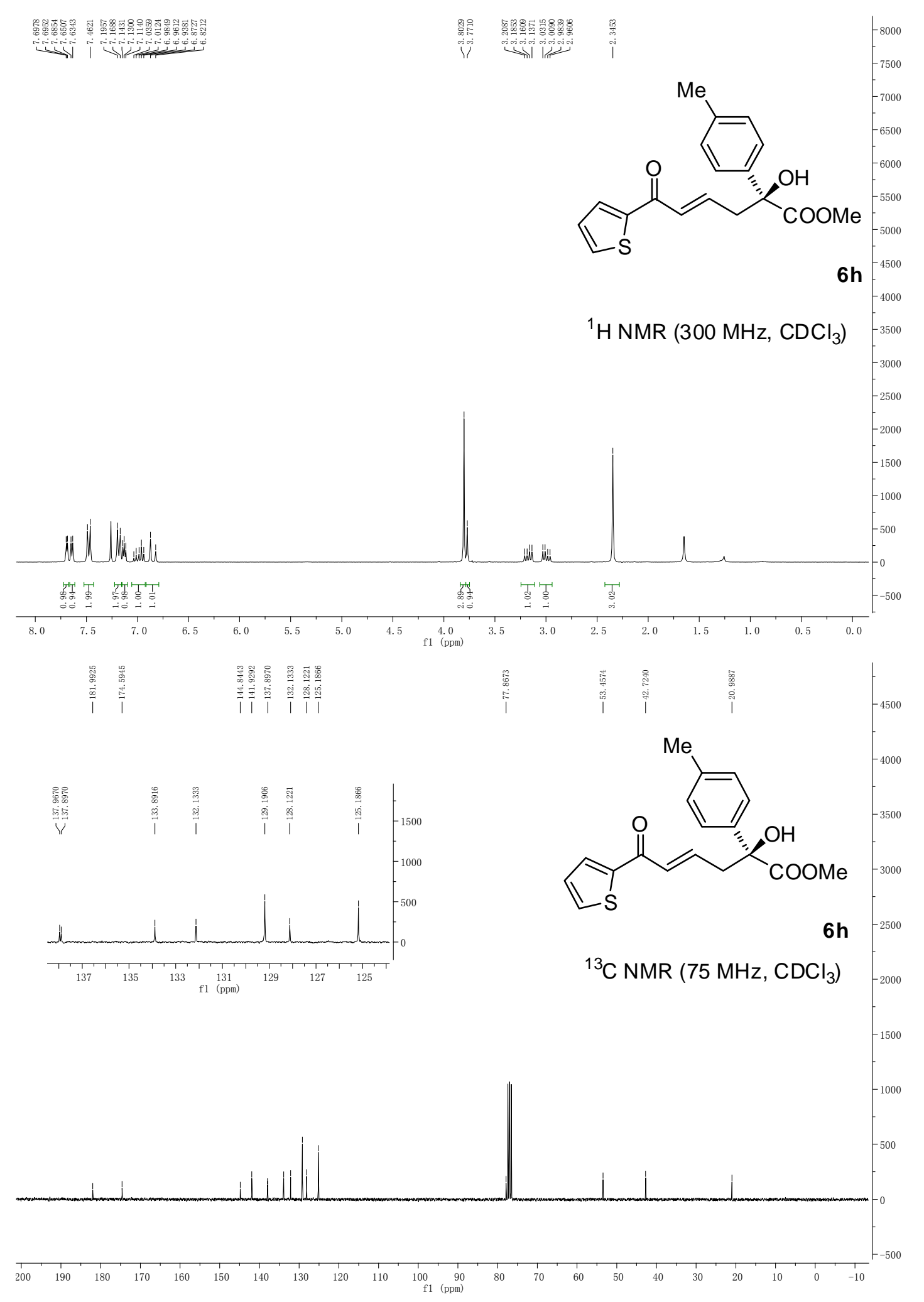




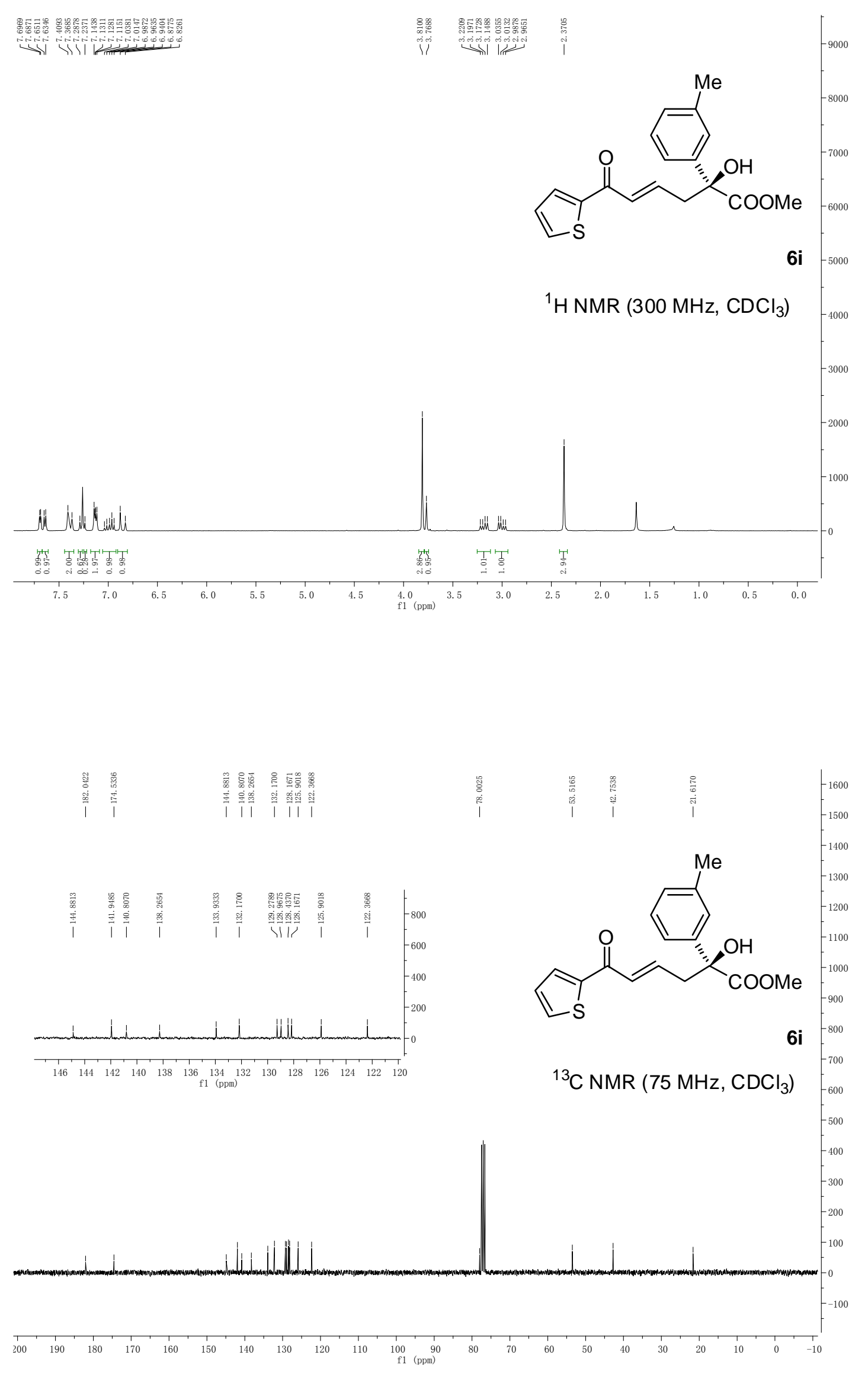




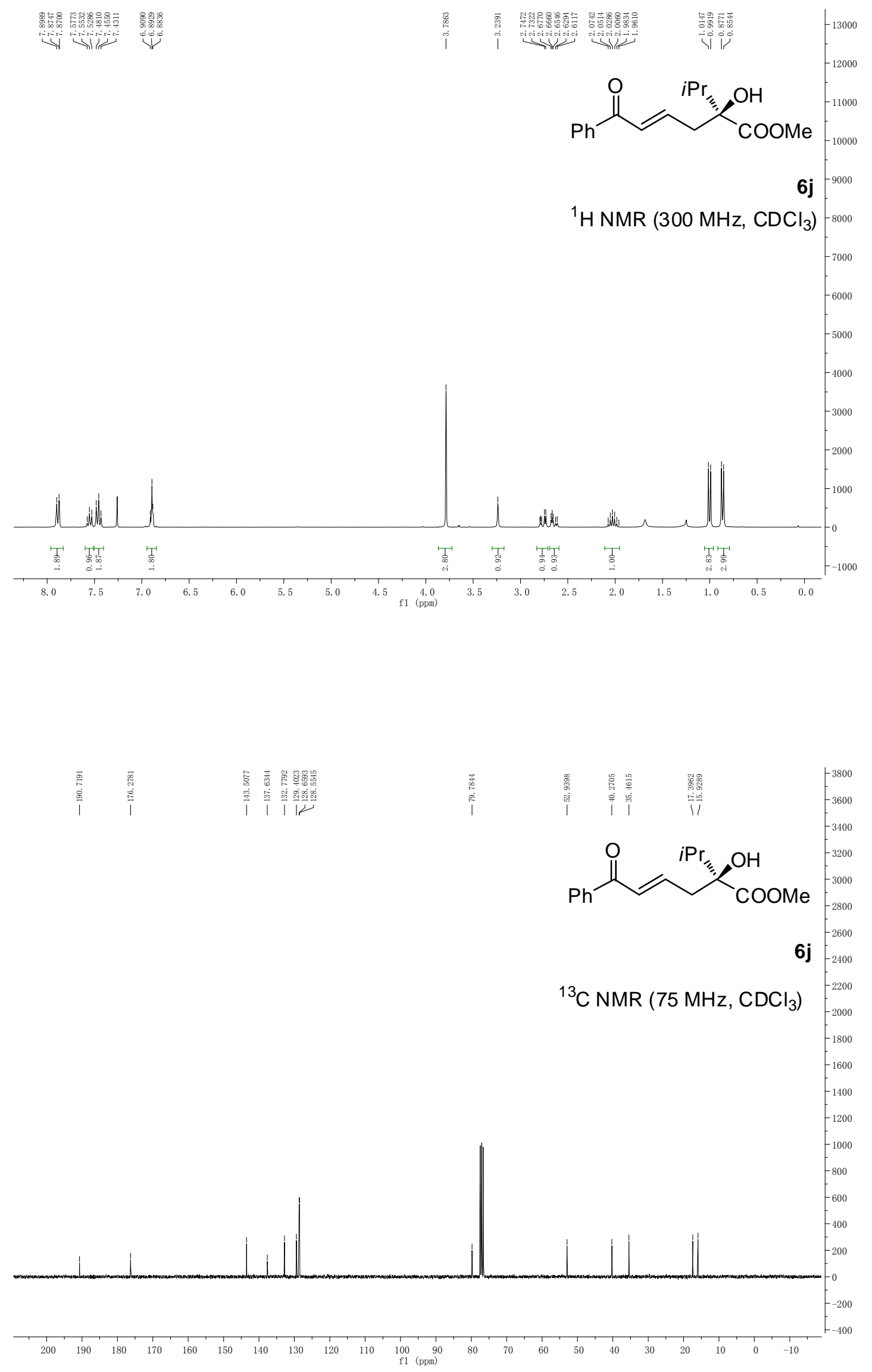




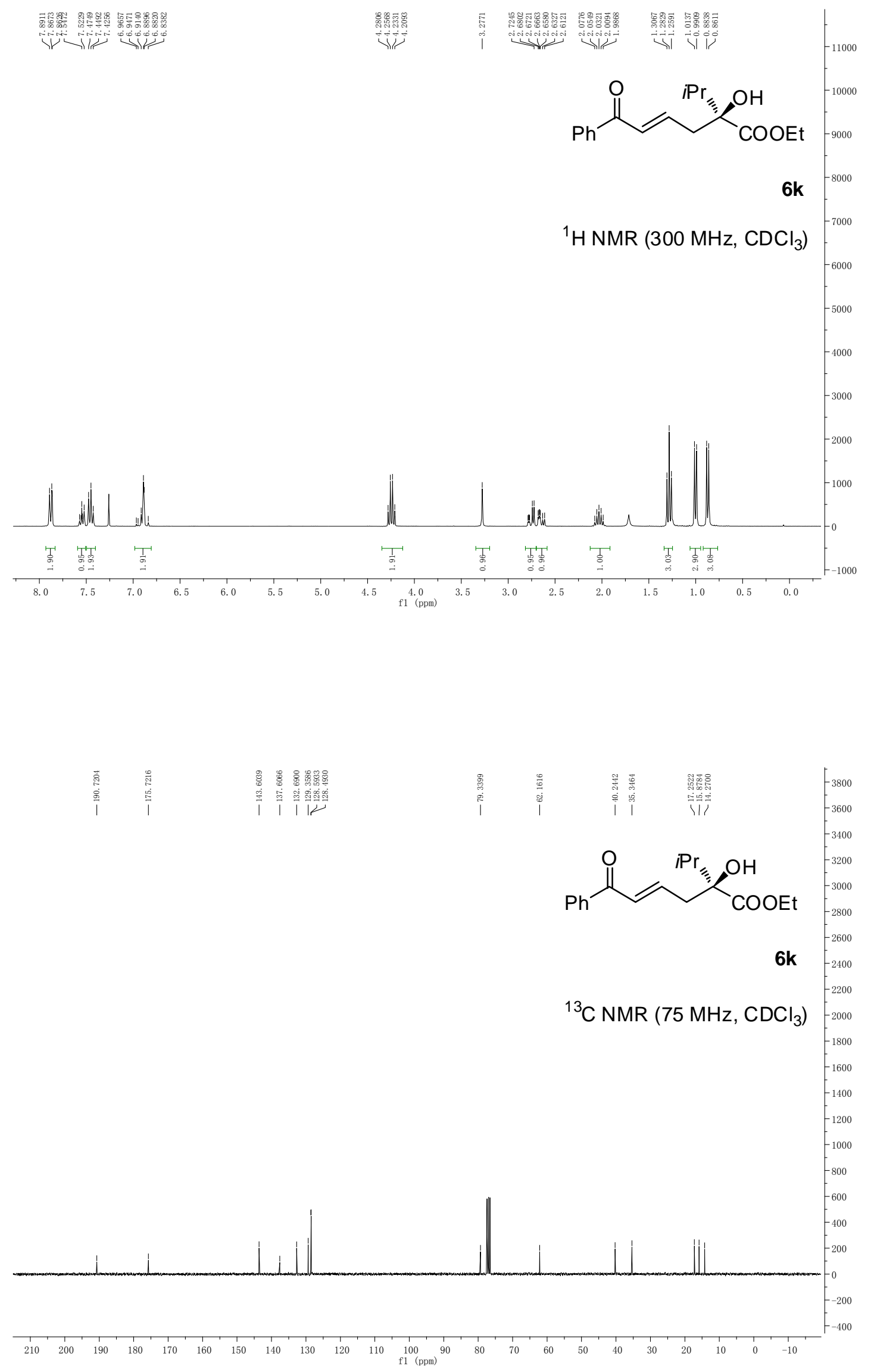




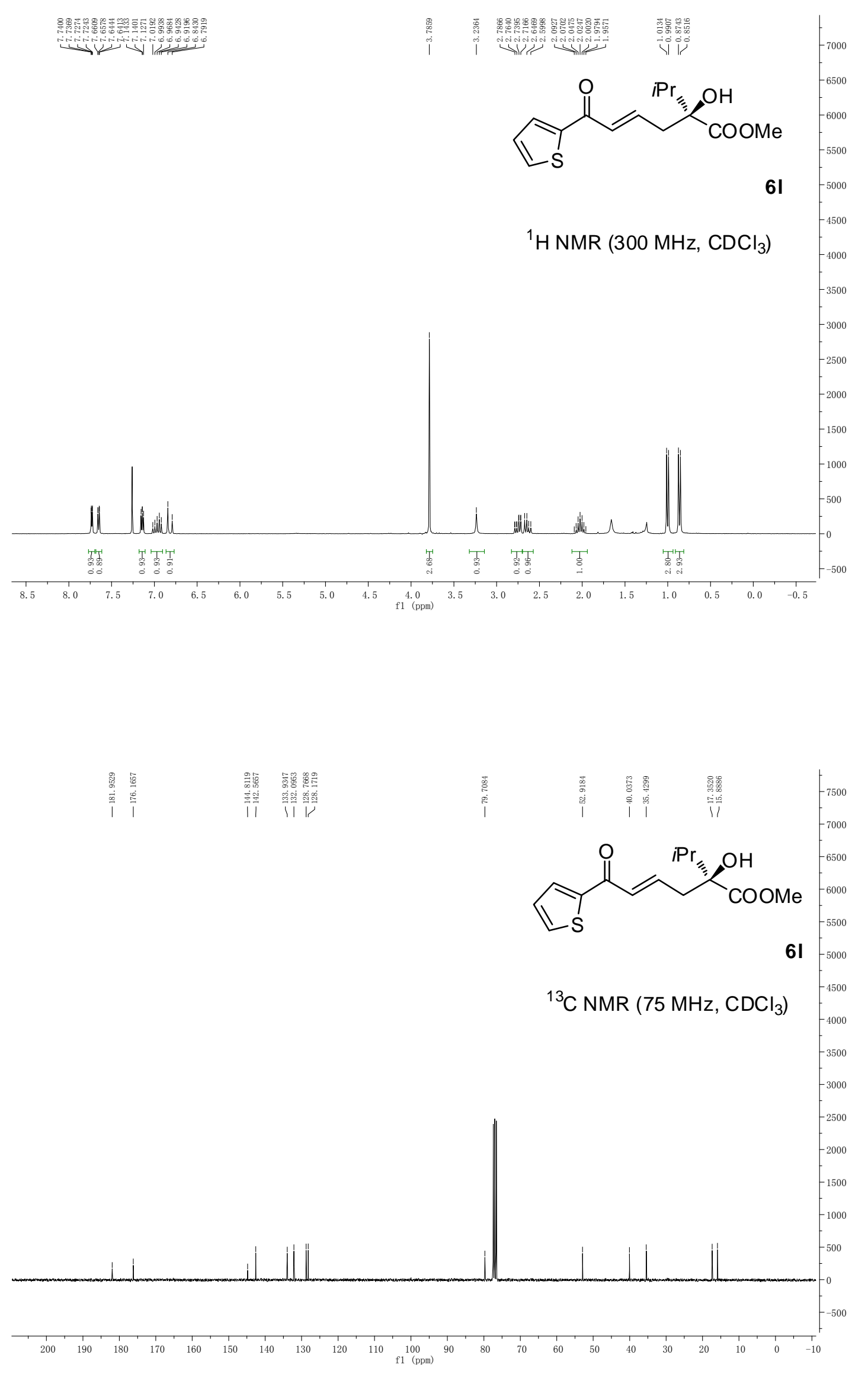



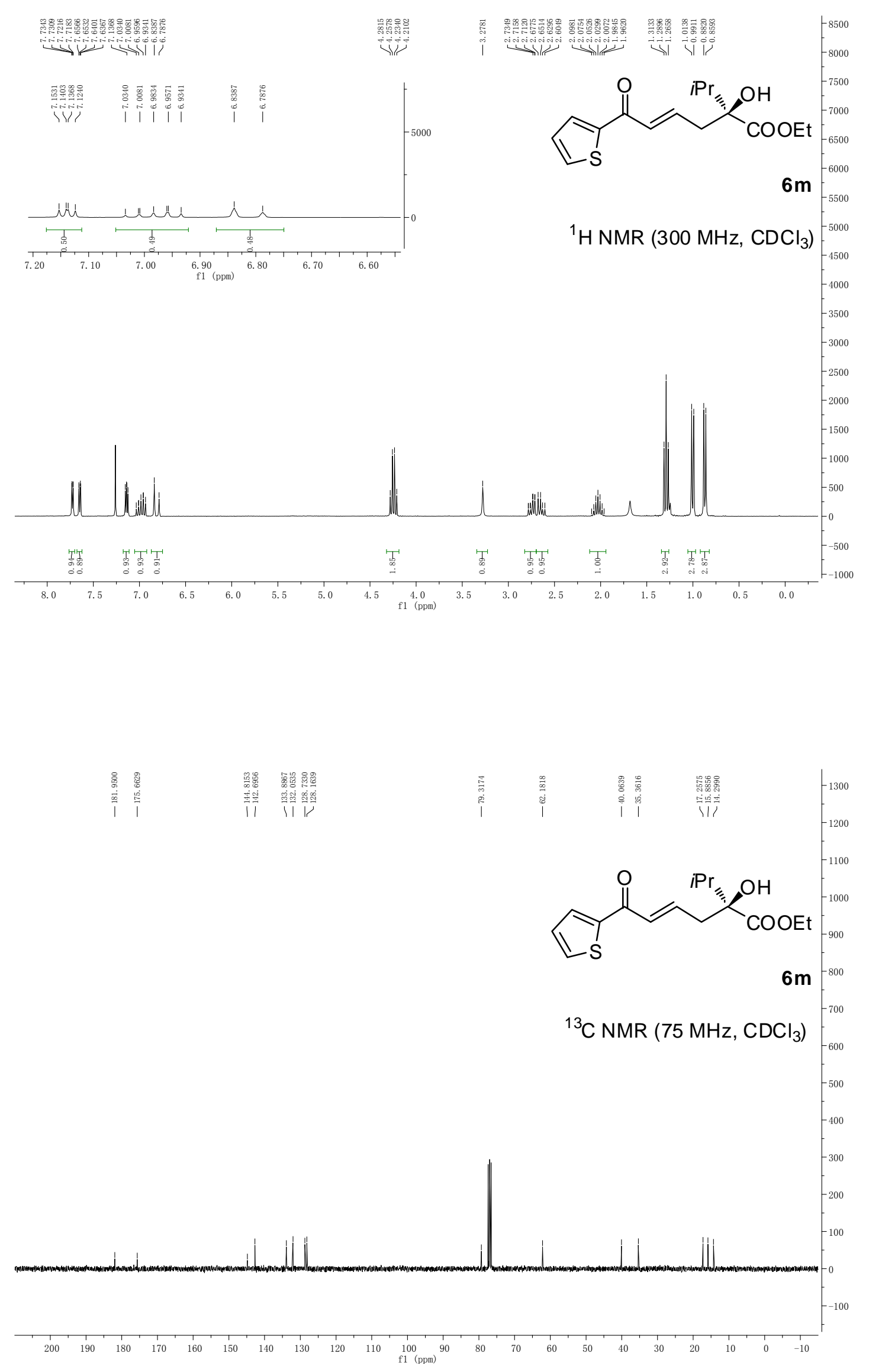


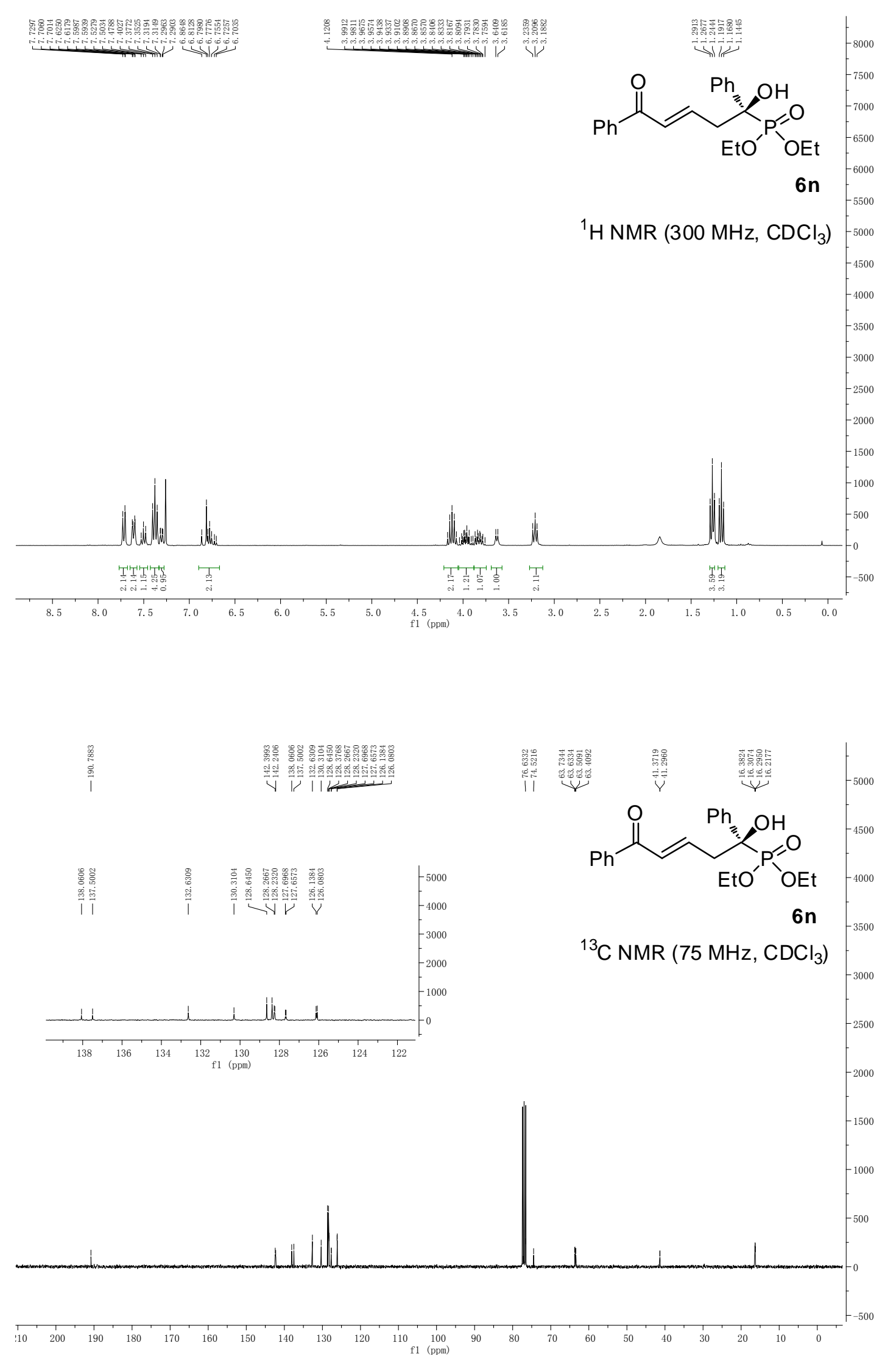




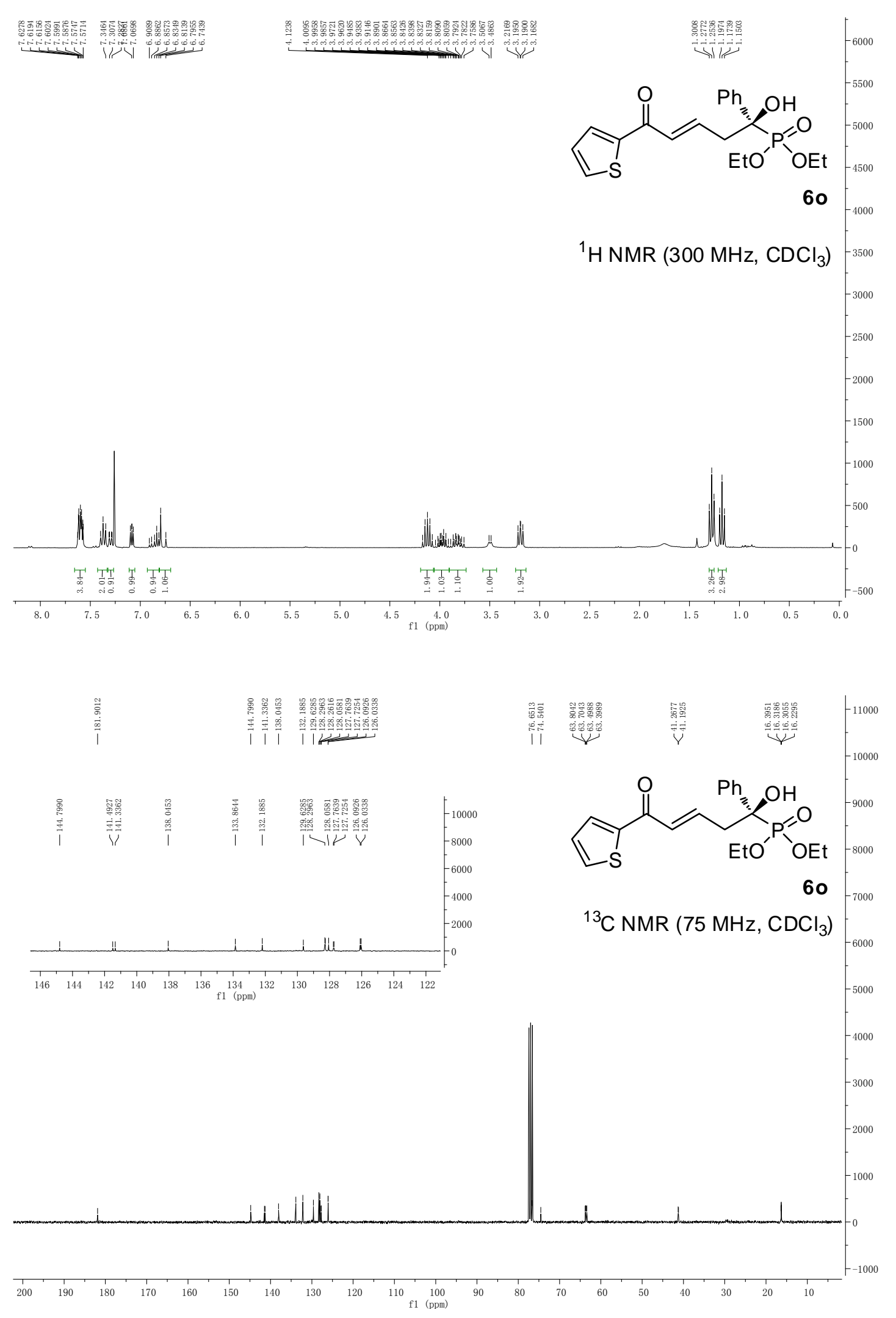




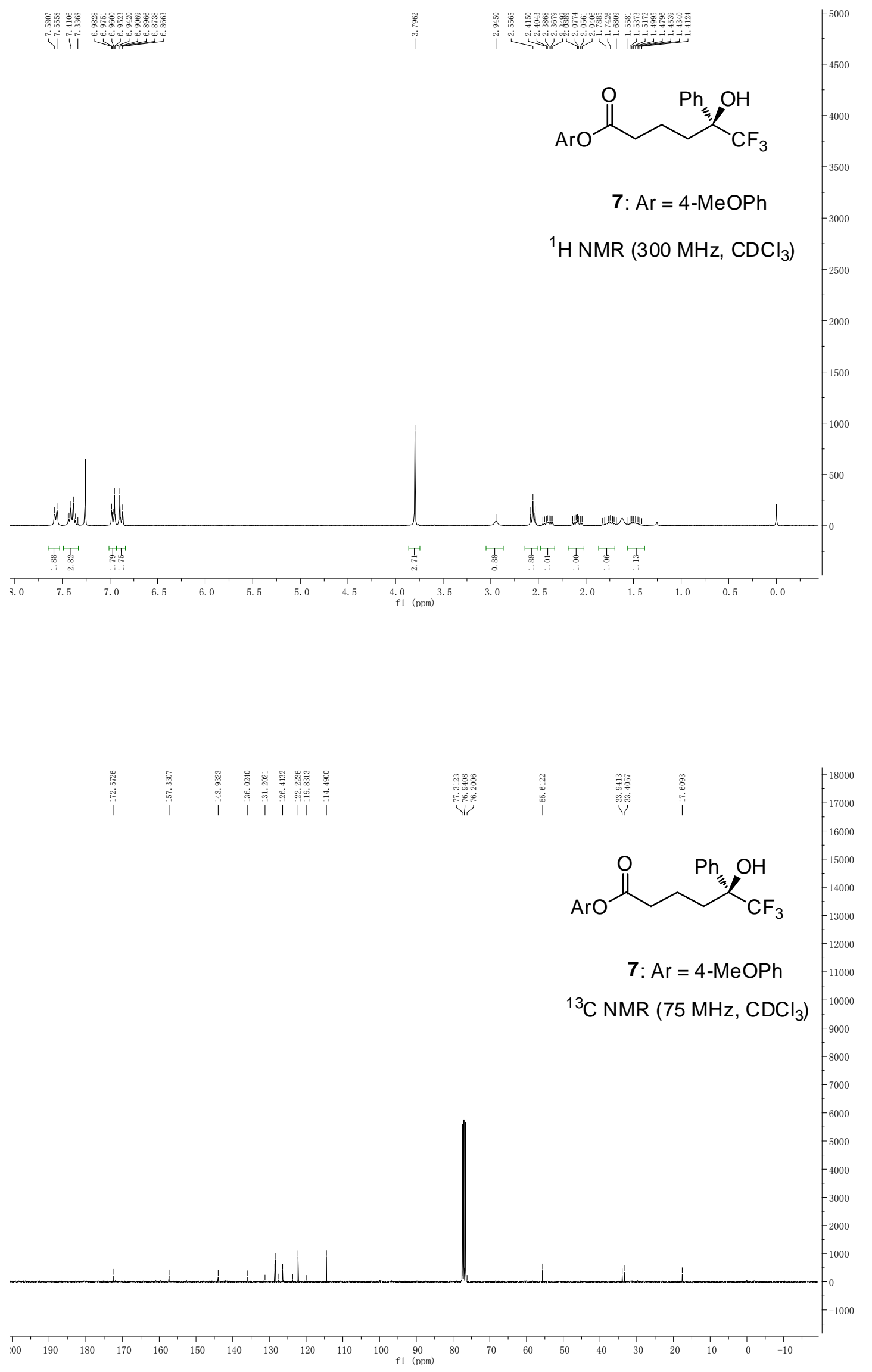



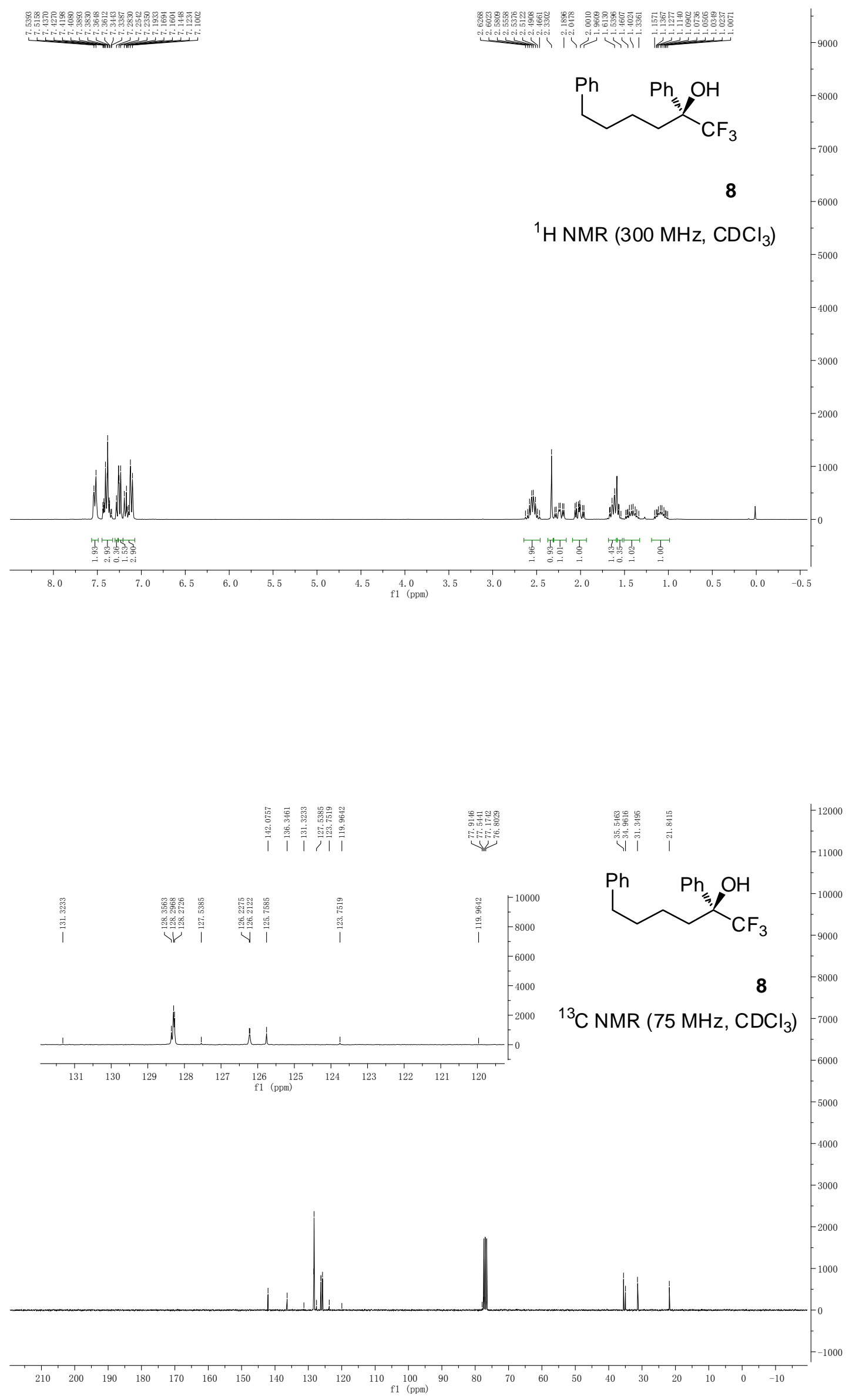


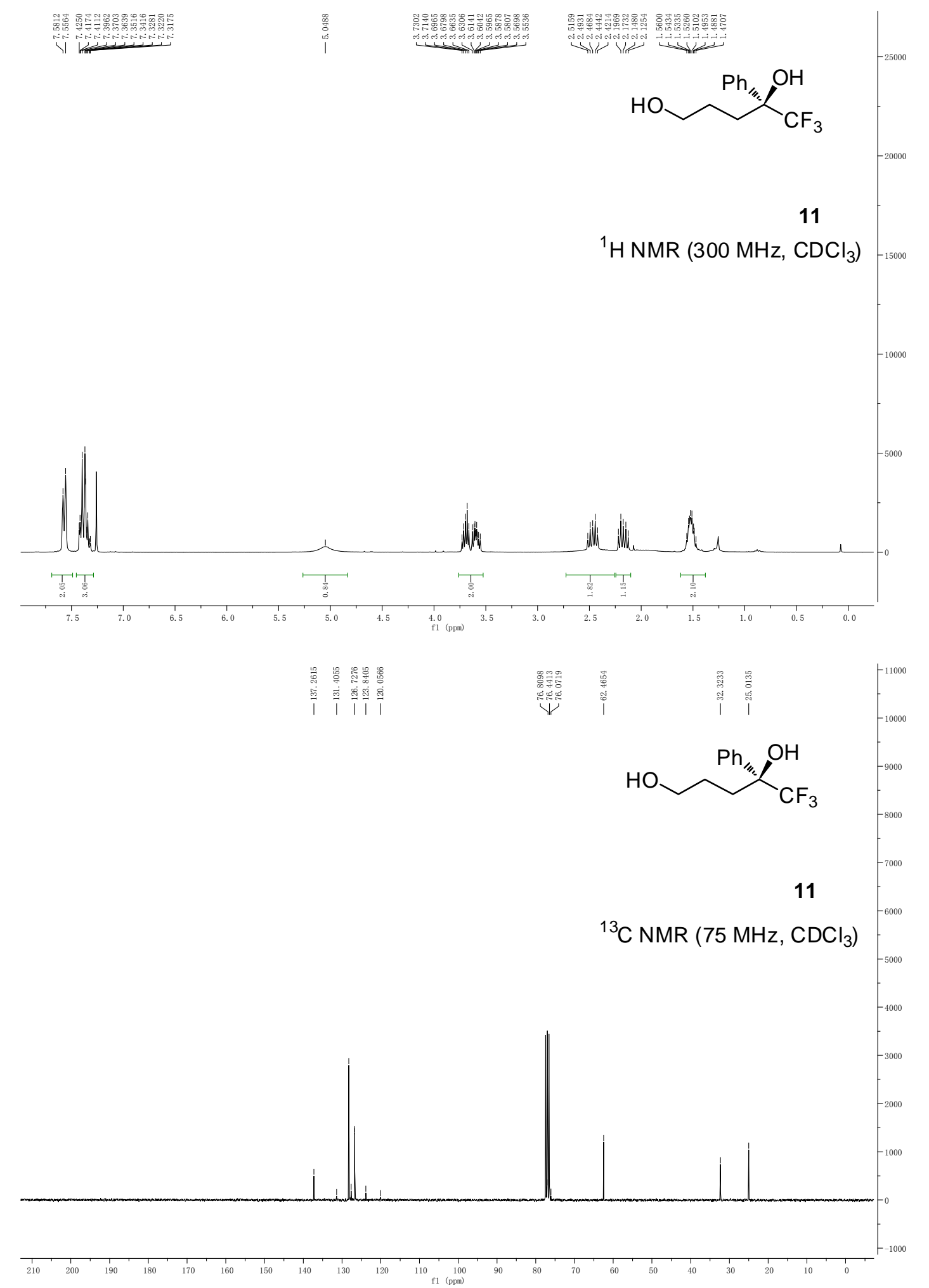




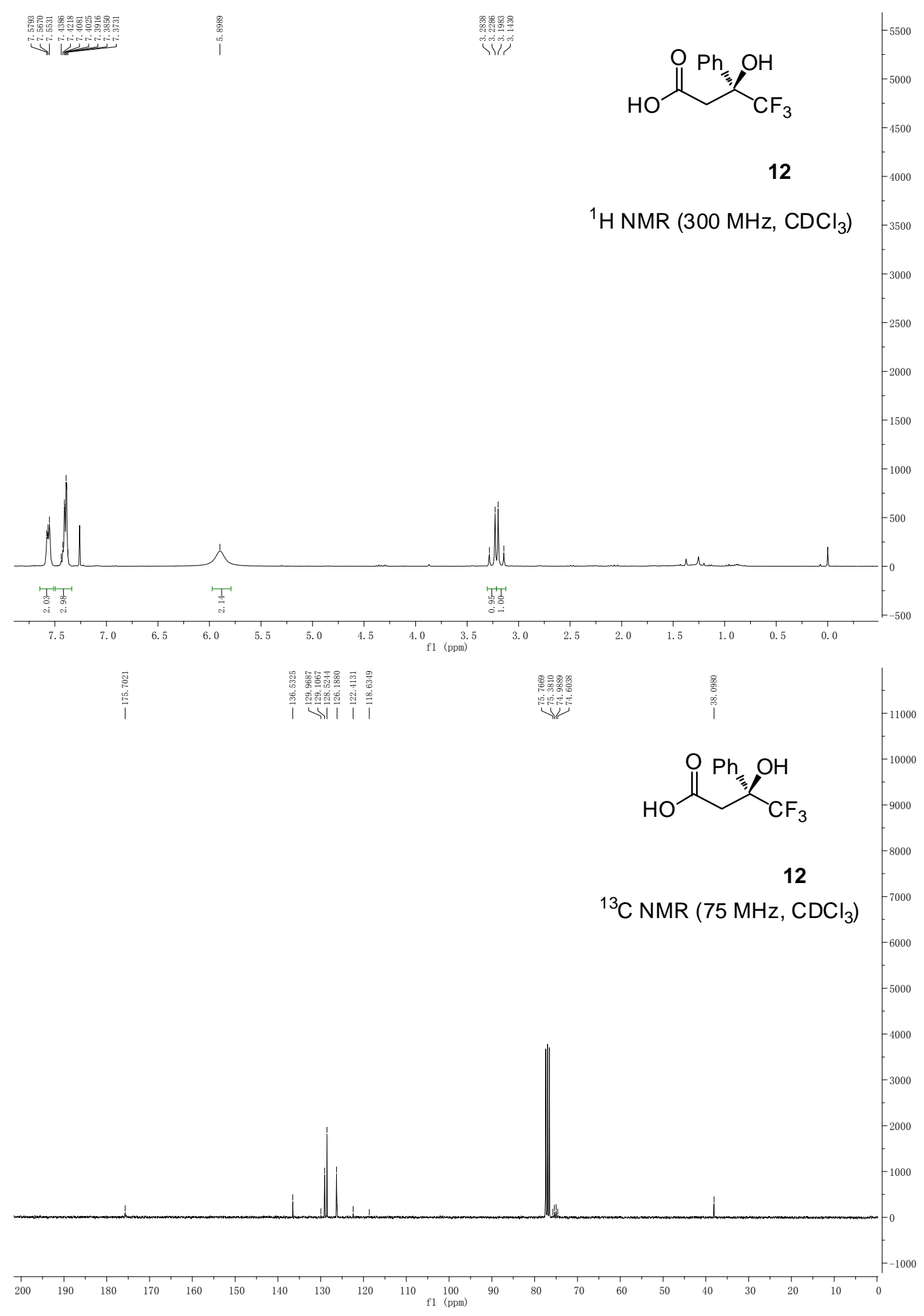




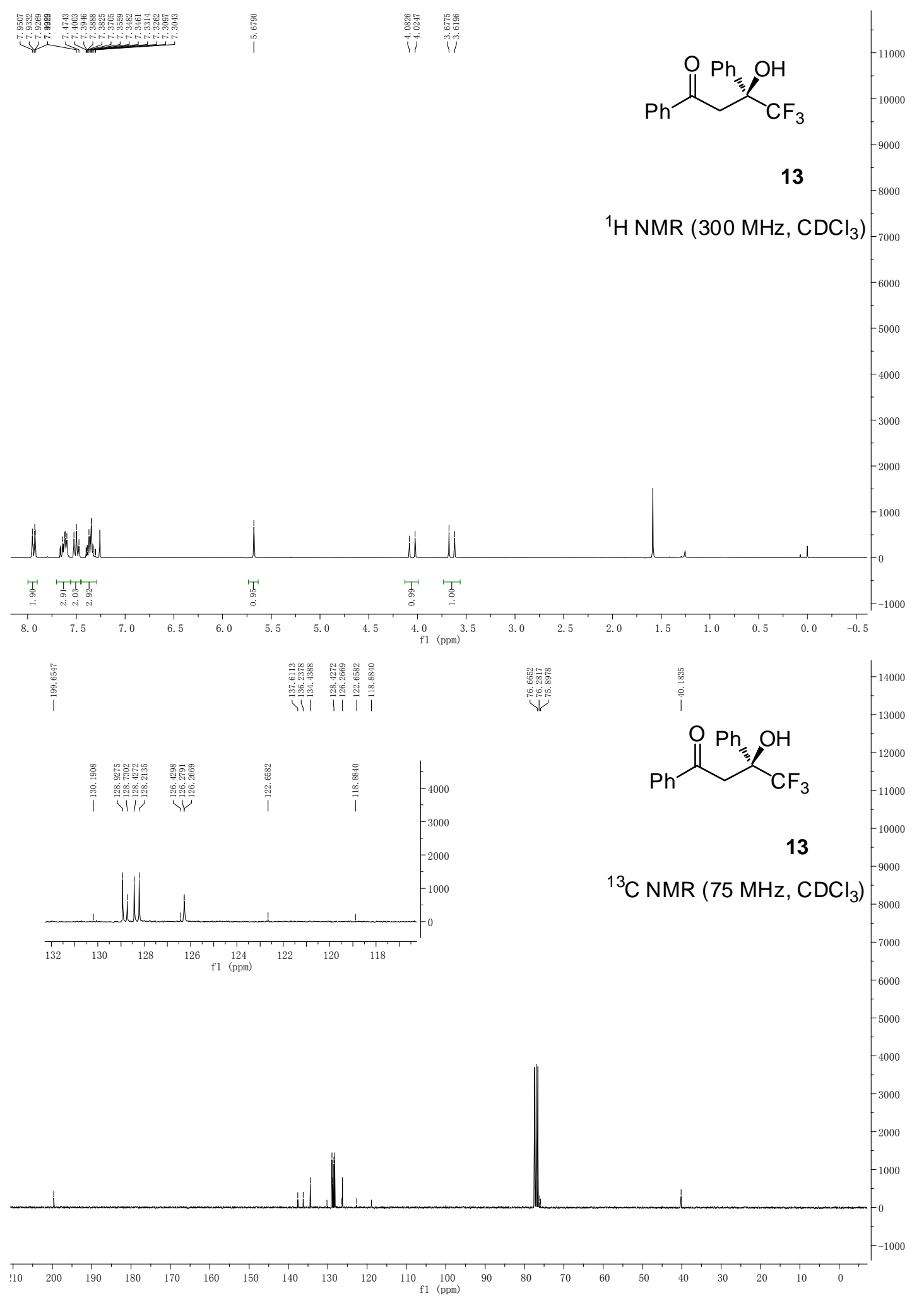




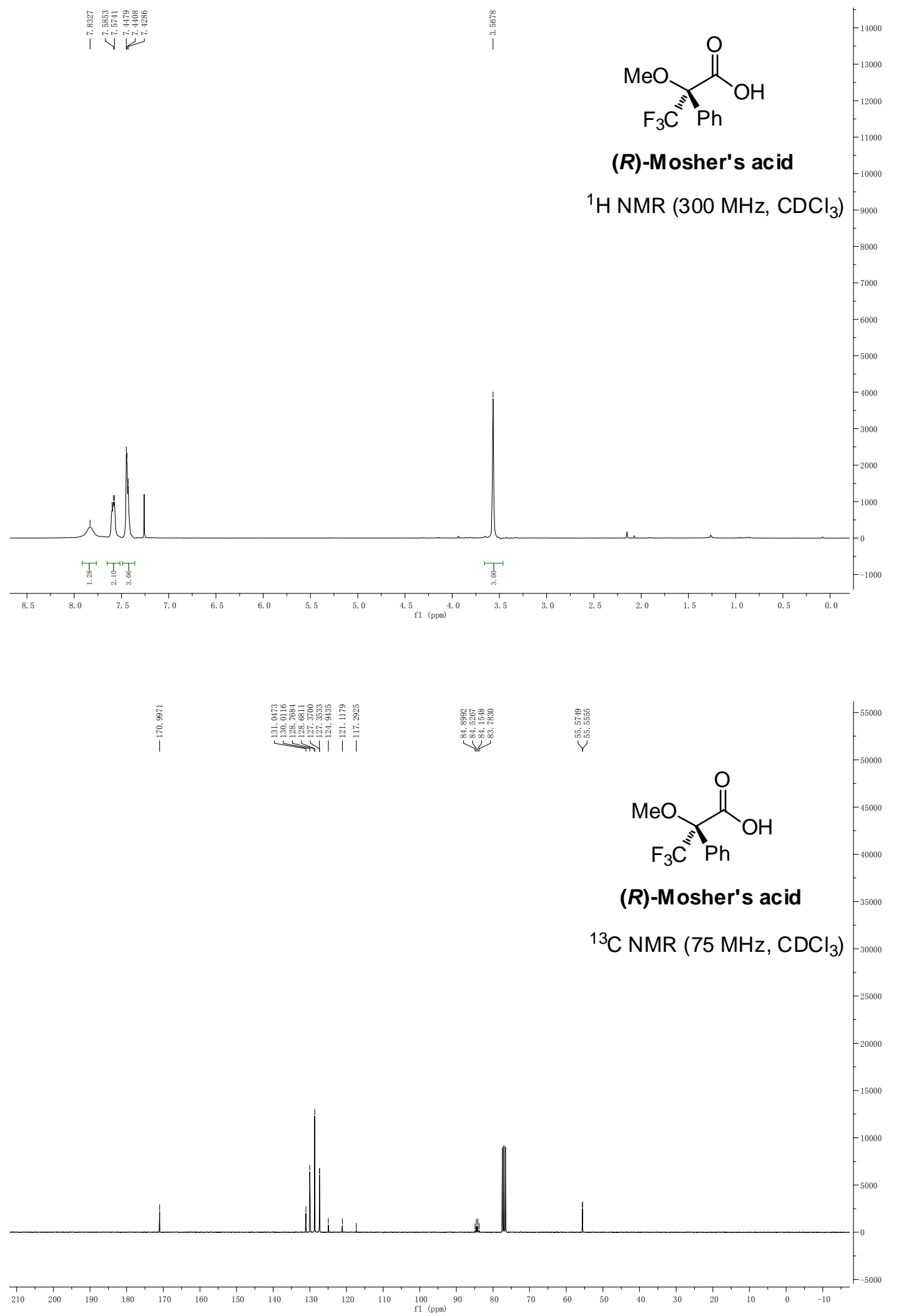

